\title{
Radiotherapy and immunotherapy
}

Citation for published version (APA):

Rekers, N. H. (2017). Radiotherapy and immunotherapy: the perfect partnership. [Doctoral Thesis, Maastricht University]. Datawyse / Universitaire Pers Maastricht. https://doi.org/10.26481/dis.20170309nr

Document status and date:

Published: 01/01/2017

DOI:

10.26481/dis.20170309nr

Document Version:

Publisher's PDF, also known as Version of record

Document license:

Unspecified

\section{Please check the document version of this publication:}

- A submitted manuscript is the version of the article upon submission and before peer-review. There can be important differences between the submitted version and the official published version of record.

People interested in the research are advised to contact the author for the final version of the publication, or visit the DOI to the publisher's website.

- The final author version and the galley proof are versions of the publication after peer review.

- The final published version features the final layout of the paper including the volume, issue and page numbers.

Link to publication

\footnotetext{
General rights rights.

- You may freely distribute the URL identifying the publication in the public portal. please follow below link for the End User Agreement:

www.umlib.nl/taverne-license

Take down policy

If you believe that this document breaches copyright please contact us at:

repository@maastrichtuniversity.nl

providing details and we will investigate your claim.
}

Copyright and moral rights for the publications made accessible in the public portal are retained by the authors and/or other copyright owners and it is a condition of accessing publications that users recognise and abide by the legal requirements associated with these

- Users may download and print one copy of any publication from the public portal for the purpose of private study or research.

- You may not further distribute the material or use it for any profit-making activity or commercial gain

If the publication is distributed under the terms of Article $25 \mathrm{fa}$ of the Dutch Copyright Act, indicated by the "Taverne" license above, 


\section{Radiotherapy and immunotherapy}

the perfect partnership

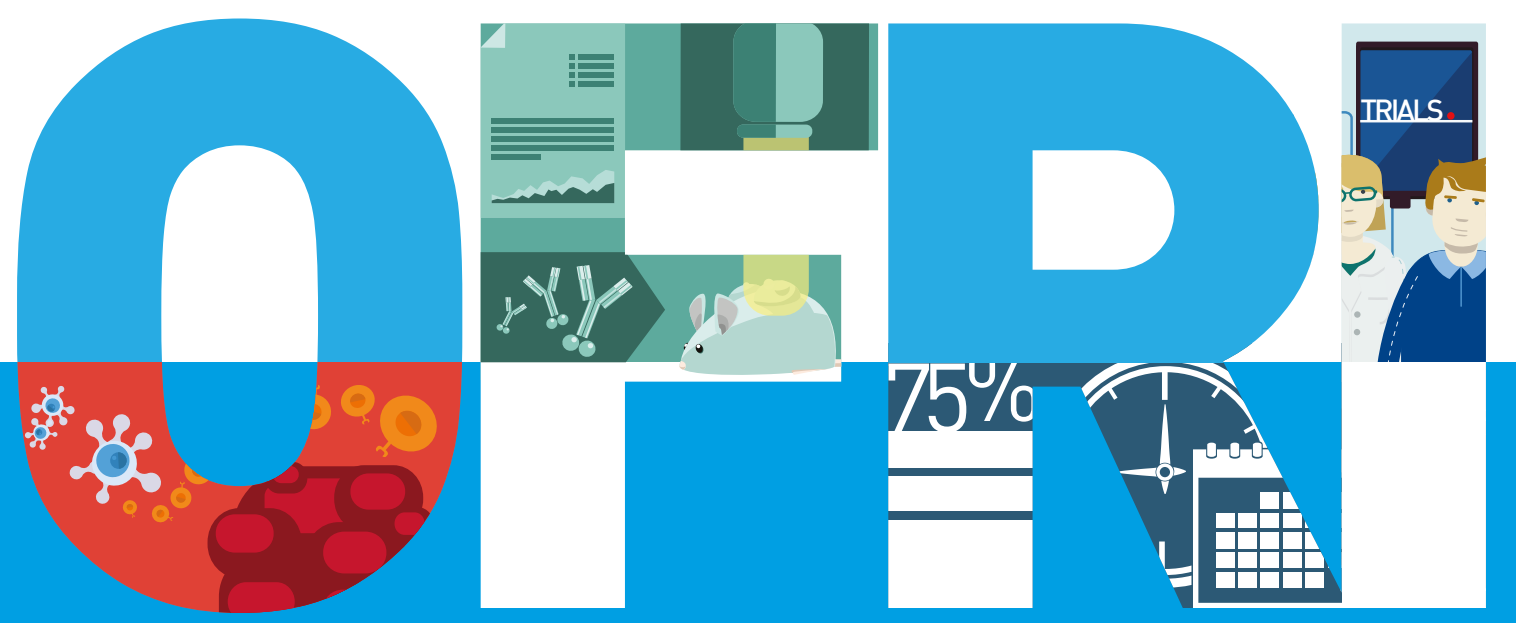

Nicolle Rekers 
Cover:

The Out-of-Field Radiolmmune (OFRI) effect

Vincent de Mees |Animating Science, Rotterdam

\section{Production:}

Datawyse | Universitaire Pers Maastricht

ISBN: 9789461596710

(C) Copyright Nicolle Rekers, Maastricht 2017

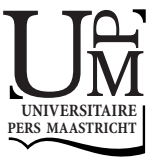




\title{
Radiotherapy and immunotherapy: the perfect partnership
}

\author{
Proefschrift \\ ter verkrijging van de graad van doctor aan de Universiteit Maastricht, \\ op gezag van de Rector Magnificus, Prof. dr. Rianne M. Letschert, \\ volgens het besluit van het College van Decanen \\ in het openbaar te verdedigen \\ op donderdag 9 maart 2017 om 12:00 uur \\ door
}

Nicolle Hubertine Rekers 


\section{Promotor}

Prof. dr. P. Lambin

Co-promotores

Dr. L.J. Dubois

Dr. A. Yaromina

\section{Beoordelingscommissie}

Prof. dr. F.C.S. Ramaekers (voorzitter)

Prof. dr. G. Bos

Prof. dr. E. Deutsch (University of Paris-Saclay)

Prof. dr. D. De Ruysscher

Prof. dr. J. de Vries (Radboud Universitair Medisch Centrum Nijmegen) 


\section{Contents}

Chapter 1 Introduction

Chapter 2 Stereotactic ablative body Radiotherapy combined with immunotherapy: present status and future perspectives

Chapter 3 Radiotherapy combined with the immunocytokine L19-IL2 provides long-lasting antitumor effects

Chapter 4 Long-lasting anti-tumour effects provided by Radiotherapy combined with the immunocytokine L19-IL2

Chapter 5 The immunocytokine L19-IL2: a perfect interplay between radiotherapy and long-lasting systemic anti-tumour immune responses

Chapter 6 Combination of radiotherapy with the immunocytokine L19-IL2: additive effect in a NK cell dependent tumour model

Chapter 7 IL2 based immunotherapies: Towards a personalized and curative anti-tumour response

Chapter 8 Radiotherapy, L19-IL2 and ipilimumab trimodal treatment: a suppressive role of PD-L1?

Chapter 9 General discussion and future perspectives

Summary

Nederlandse samenvatting

Valorisation addendum

Acknowledgements / dankwoord

Curriculum Vitae

List of publications 

Chapter

Introduction 


\section{CANCER IN THE SOCIETY}

The World Health Organization has recognized cancer as a leading cause of morbidity and mortality worldwide, with an effective 14 million new cases and 8.2 million annual deaths in 2012. Furthermore, cancer incidence is expected to increase by approximately $70 \%$ over the next twenty years (1). There are more than 100 different types of cancer and the ultimate aim of anti-cancer therapy is to provide a personalized treatment that targets and cures (systemic) disease. There are three main cancer treatment modalities: surgery, radiotherapy and chemotherapy. Surgery physically removes malignant lesions, radiotherapy targets a radiation dose towards the tumour and conventional cytotoxic chemotherapeutic agents usually kill rapidly dividing cells in the body by interfering with cell division. A fourth new treatment modality, immunotherapy, represents the most promising approach since the development of the first chemotherapies (2). The general principle of immunotherapy is the modulation of a patient's own immune system to target their cancer. The immune system of all patients is as unique as their tumour, and therefore these therapies may hold great promise. Immunotherapies have the potential to target the invisible (micro)metastases and offer long-term protection (3). New therapies are under development, and frequently several of these four treatment modalities are combined in order to achieve the optimal anti-cancer treatment.

Cancer development - A role for the immune system

In 2000, Hanahan and Weinberg published a review article explaining six biological capabilities acquired during the multistep development of human tumours (sustaining proliferative signalling, evading growth suppressors, resisting cell death, enabling replicative immortality, inducing angiogenesis and activating invasion and metastasis), named the six hallmarks of cancer (4). However, for cancers to grow progressively they must have evaded the anti-tumour immune response. Indeed, in the past decade it became more and more clear that the immune system has a pivotal role in the prevention of tumours, by identifying and eliminating tumour cells on the basis of their expression of tumour-specific (neo)antigens or molecules induced by cellular stress (5), and therefore the 'evading of immune destruction', was added as an additional hallmark in their updated review published over a decade later (6). This hallmark, also called immuno-editing, is a process divided into three phases: elimination, equilibrium and escape (Fig. 1). 

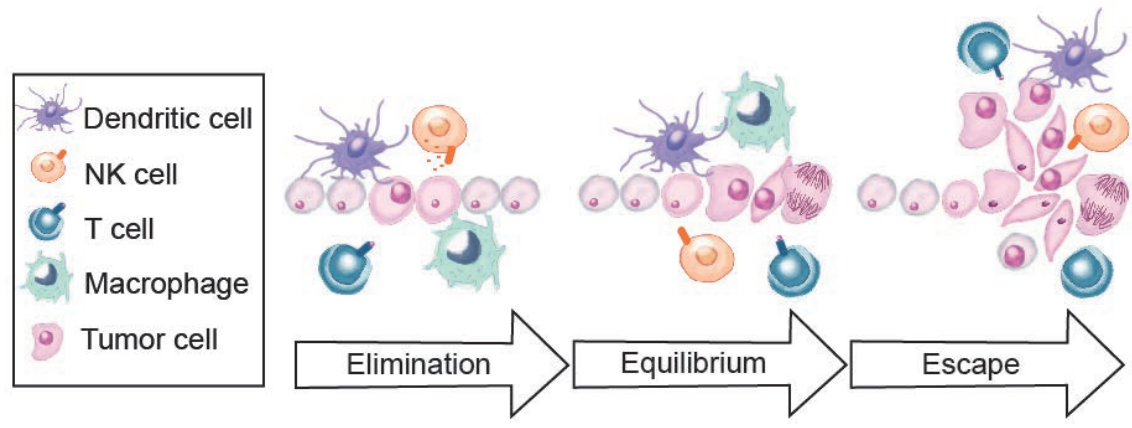

Figure 1: The process of immuno-editing. In the first phase, the immune system recognizes and eliminates malignant cells. In the second phase, tumour cells can develop in different variants (equilibrium), eventually escaping (third phase) the killing mechanism of the immune cells (adapted from (7)).

In the first phase, the immune system can detect and eliminate tumour cells. Both innate and adaptive immune cells actively prevent neoplastic development in this first phase. Innate immune cells mediate an immediate tumour cell attack by producing cytokines that can directly lyse tumour cells (natural killer (NK) cells) (8) and by capturing tumour debris and antigens (including dendritic cells (DCs), neutrophils and macrophages) (9). Eosinophils and basophils are thought to be involved in attacking large antibody-coated parasites and mast cells can trigger local inflammatory responses in response to antigens and are well known to cause allergic reaction (9). In patients and animal models, the loss of proper NK function has been associated with an increase in the incidence of a variety of cancers (10). As members of the innate immune system, NK cell functions are tightly regulated by a balance between activating and inhibitory signals. These signals are provided by receptors expressed at their cell surface, enabling NK cells to recognize and spontaneously kill target cells, such as virus infected and tumour cells, without prior sensitization. Therefore, these abnormal cells trigger NK effector functions (cytotoxicity, cytokine production and proliferation) directly, either through the loss of $\mathrm{MHCl}$ class molecules (i.e. loss of self-identification) that can otherwise bind to inhibitory receptors on the NK cells or by upregulating of ligands that activate NK cells to overcome these inhibitory signals (Fig. 2) (11).

Several cell types of the innate immune system, including macrophages and DCs, can perform the recognition, capturing and elimination of foreign cells. As with NK cells, recognition occurs via an interaction with cell-surface pattern recognition receptors that are able to recognize PAMPs (pathogen-associated molecular patterns) conserved among microbes and DAMPs (damage-associated molecular patterns) released from and expressed on tissue injuries. Macrophages were initially known to pick up and eliminate tumour cells (12), however, they have also been shown to be recruited to tumours and correlate with poor prognosis (13). The function of DCs seems to be more straight-forward as they capture, process and eliminate foreign cells (including tumour 
cells) in their role as innate immune cell, and in addition, they have an important role in the initiation of the adaptive immune response. After processing the antigen, DCs have the ability to migrate from the antigen capturing side (e.g. the tumour) into draining secondary lymphoid organs, where they encounter naïve helper $\left(C D 4^{+}\right)$and cytotoxic $\left(\mathrm{CD}^{+}\right) \mathrm{T}$ cells, triggering their proliferation and eventually migration of antigen-specific T cells into peripheral tissues, thereby starting the adaptive immune response $(14,15)$.

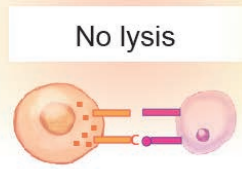

Healthy cell

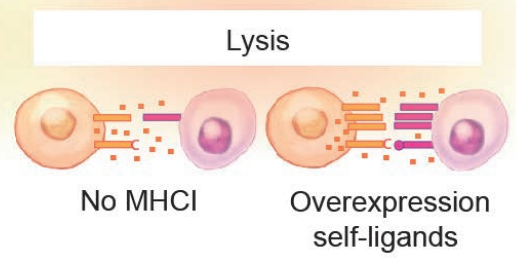

Figure 2: Regulation of NK cells. Loss of self-identification through a lack of $\mathrm{MHCl}$ expression or the upregulation of activating ligands can both overcome inhibitory signals of healthy cells and activate NK function (adapted from (11)).

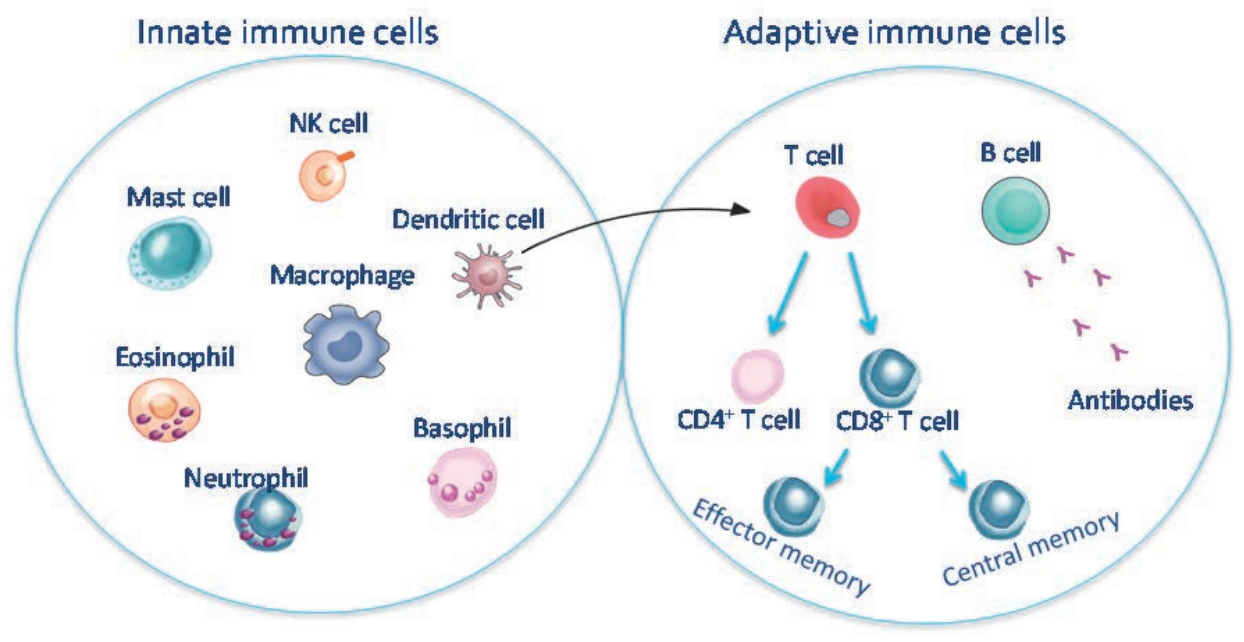

Figure 3: Overview of cells from the innate and adaptive immune system. Cells from the innate immune response target and destroy foreign cells immediately after recognition. Dendritic cells have a crucial role in capturing of debris and initiating the adaptive immune response. T cells from the adaptive immune response can develop into $\mathrm{CD}^{+}$(helper) and $\mathrm{CD}^{+}$(cytotoxic) T cells, which have the potential to rapidly develop into memory $T$ cells able to kill target cells upon recognition (adapted from (67)). 
Tumour (neo)antigens derived from mutations associated with carcinogenesis (16) have the potential to be recognized by $T$ cells of the adaptive immune system. Upon recognition of a matching tumour antigen, $T$ cells have the ability to attack and destroy these cells, completing the process of elimination. Indeed, activated anti-tumour T cells, endowed with antigen specificity (and memory), are required to achieve complete and long-lasting tumour clearance (17). However, the regulation of $T$ cell responses is a complex process as a result of a sophisticated balance consisting of stimulatory and inhibitory signalling pathways, chemokines and cytokines. The first stage (elimination of tumour cells) can be unsuccessful, often because tumour cells can progress in different variants (during equilibrium) eventually escaping the killing mechanism of immune cells (Fig. 1 (5)). This large variety of tumour cells is able to interact and manipulate their environment (also called the tumour microenvironment), thereby recruiting tumour supporting fibroblasts, blood vessels and (regulatory) immune cells $(18,19)$. In order to effectively execute their anti-tumour role, T cells of the adaptive immune system require additional costimulatory signals and stimulatory cytokines, such as interleukin-2 (IL2), secreted by antigen-stimulated $\mathrm{CD}^{+}$and $\mathrm{CD}^{+}{ }^{+}$cell, NK cells and activated DCs $(20,21)$. In order to maintain self-tolerance and to be able to control the duration and amplitude of a physiological immune response with minimal collateral tissue damage, $T$ cells additionally have the possibility to activate several inhibitory pathways (22). Both of these principles of the anti-cancer immune response can be manipulated: the addition of stimulatory cytokines has the potential to make the response stronger and the addition of the so-called checkpoint inhibitors can combat the inhibitory pathways.

\section{Checkpoint inhibitors - releasing the brakes of inhibitory pathways}

Despite the immune escape, solid tumours are often highly infiltrated by several immune cells. There is evidence showing a positive association between intra-tumoural lymphocytes and increased survival in patients with solid tumours (23). Therefore, the search for mechanisms involved in the dysfunction and exhaustion of the anti-tumour immune response (i.e. ways to combat the inhibitory pathways) and ways to restore and manipulate this broad and unique process, has gained a lot of interest over the last decade. Indeed, the field of immunotherapy was recognized as breakthrough of the year 2013 by Science (2), because of their ground-breaking findings (24-27) and their totally different way of treating cancer: by targeting the tumour indirectly via the immune system. Recently, profound progress in immunotherapy research was achieved in the field of checkpoint inhibitors (22). Among recently FDA (Food and Drug Administration) approved immune checkpoint inhibitors are anti-CTLA4 (ipilimumab) and the anti-PD1/PD-L1 inhibitors (atezolimab, pembrolizumab and nivolumab), all releasing the brakes 
elicited by the immune system or tumour cells and showing encouraging durable tumour regression in a percentage of (metastatic) cancer patients (22).

\section{(Targeted)-IL2 - pushing the accelerator using stimulatory cytokines}

To initiate an anti-tumour immune response, immune cells need to recognize tumourspecific (neo)antigens, they need to be activated (without developing an exhausted phenotype) and they need to be stimulated by the correct repertoire of immunostimulatory cytokines. One cytokine, IL2, is secreted by activated $\mathrm{CD} 4^{+}, \mathrm{CD}^{+}$, NK and dendritic cells and it can stimulate cells that express the IL2 receptor (CD25). IL2 is known to stimulate $\mathrm{CD}^{+} \mathrm{T}$ cell growth and differentiation and represents the first FDA approved immunotherapy that mediates the regression of large number of human cancers. It is also an important factor responsible for the maintenance of $\mathrm{CD}^{+}$ regulatory $\mathrm{T}$ cells and plays a role in $\mathrm{CD}^{+}{ }^{+} \mathrm{T}$ cell differentiation and NK cell activation $(21,28)$. Despite the great potential of IL2 treatment in, for example, metastatic melanoma, its clinical application remains restricted due to its short half-life, making it necessary to give high doses to achieve optimal immune-modulatory effects but causing severe toxicities (hypotension, vascular leak syndrome and heart toxicities) (29-31). A way to circumvent toxicities caused by the high dose systemic IL2 treatment is intratumoural IL2 administration (21) or through selective delivery of IL2 in the form of a cytokine fusion protein, or immunocytokine (32). L19-IL2 is such an immunocytokine, containing the monoclonal antibody L19 in a diabody format, which recognizes the extra-domain B (ED-B) of fibronectin, a marker associated with tumour angiogenesis (33). ED-B is present in newly formed vasculature structures of most solid tumours and absent in healthy tissues (with exception of tissues of female reproductive cycle and during foetal development), making it an interesting tumour (microenvironment) targeting protein. Indeed, previous studies using L19 for imaging and targeted (radio)immunotherapy, have shown that L19 targets the tumour vasculature (34-36). Moreover, in a phase I clinical study in patients with melanoma or renal cell carcinoma, the administration of L19-IL2 alone or combined with chemotherapy (decarbazine), was safe and showed clinical activity $(37,38)$. Currently, a phase II study is ongoing investigating the efficacy of L19-IL2 combined with L19-TNF (tumor necrosis factoralpha) in stage III and IV melanoma patients (NCT02076633).

\section{Radiotherapy and immunotherapy - the best of two worlds}

Although recent developments in immunotherapy research are extremely promising, recent data suggest that greater success can be achieved by combining immunotherapeutic approaches with radiotherapy $(39,40)$. Radiotherapy $(\mathrm{RT})$ is one of the major treatment options in cancer management and approximately $52 \%$ of all cancer patients receives RT during their treatment (41). For decades, the direct and local 
effects of RT on tumour cells, depending on DNA damage and the intrinsic repair capacity of irradiated cells (42), was the focus of RT-related research. Additionally, RT causes an immunogenic death of cancer cells characterized by calreticulin (CRT) translocation to the surface of dying tumor cells (43) together with high-mobility group box-1 (HMGB-1) and adenosine triphosphate (ATP) release, which promotes uptake and cross-presentation of released tumor (neo)antigens by DCs to T cells in the draining lymph node (16, 44-46) (Fig. 3). It has been shown that RT provides key components to initiate an immune response and to convert the irradiated tumour into an effective in situ personalized tumour vaccine (47). The immune response initiating role of RT, the formation of an in situ personalized vaccine and immune modulating effects triggered by RT, can in theory form the basis for novel personalized 'super' therapies, when combined with immunotherapy.

On the other hand, the administration of an immunotherapy has also great potential in transforming RT into a systemic and long-lasting therapy, a phenomenon known as the abscopal effect (50-54). Because its clinical appearance is sporadic (55) and it has been established to be immune-mediated (56-61), the rational of transforming the local RT treatment into a systemic therapy, has recently gained a lot of interest (62). A strong systemic anti-tumour response, could in theory target tumours (and tumour cells) outside the RT field, providing a treatment for (micro-)metastatic disease (Fig. 3). Furthermore, once irradiated, tumour antigens are successfully converted into an in situ vaccine eliciting tumour-specific $T$ cells, the host can be endowed with immune memory. An increase in long-term survival can be derived from successful immunization against the primary tumour using radiotherapy $(63,64)$, therefore these memory $T$ cells have the potential to form protection against a pleiotropy of tumour associated antigens for the life time of a host (65). To conclude, the RT-induced immunogenic cell death mechanisms initiating an anti-tumour immune response have the potential to improve and personalize available immunotherapeutic approaches and these have the potential to transform localized RT into a systemic and long-lasting therapy (Fig. 3). In other words, RT has the potential to make the therapeutic effects of immunotherapy (including L19-IL2) more targeted and immunotherapy has the potential to make RT effects more systemic. Combining both therapies creates a diversity of treatment opportunities and possibilities without searching for new targets and therapies. 


\section{OUTLINE OF THE THESIS}

In this dissertation we investigate the immune initiating effects of RT in combination with L19-IL2, a highly promising and targeted way to activate immune cells. The overall aim of this dissertation was to investigate the possibilities of transforming the effects of local radiotherapy into a systemic treatment using the immunocytokine L19-IL2 (Fig. 4).

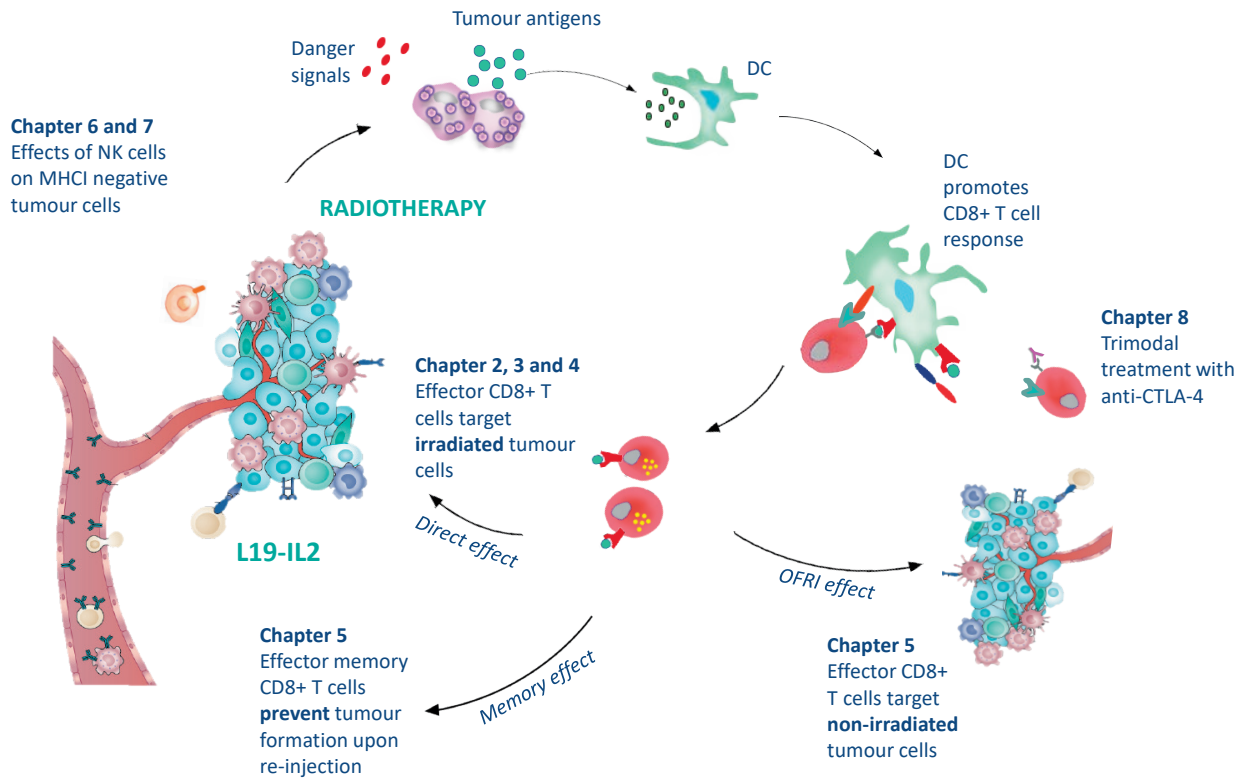

Figure 4: Combining radiotherapy with L19-IL2, a schematic overview of the supposed hypotheses of this dissertation. Radiotherapy has the potential to initiate an anti-tumour immune response and immunotherapy (L19-IL2) has the potential to increase this anti-tumour immune response systemically (modified $(48,49)$ ).

A review article (chapter 2) explores the new possibilities of bimodal treatment combining (stereotactic) RT with immunotherapeutic approaches and gives an overview of recent (ongoing) preclinical and clinical studies. We describe the potential mechanistic synergy between both treatment modalities and explain the large variety of possibilities. In chapter 3 the possible synergy between RT and the ED-B targeted immunocytokine L19-IL2 is investigated. We hypothesized that the initiated anti-tumour immune response of RT can be enhanced when combined with L19-IL2 and that this effect is dependent on the expression of ED-B. For this purpose, 3 different tumour models with varying ED-B expression levels were used. Furthermore, we investigated if the therapeutic effect of RT combined with L19-IL2 is dependent on the RT dose and if a causal relationship exists with the amount of $\mathrm{CD}^{+}$cytotoxic $T$ cells by depleting these $T$ cells with specific monoclonal anti-CD8 ${ }^{+}$antibody. Chapter 4 gives a summary of the study described in chapter 3 , and places these findings into a broader perspective. In 
chapter 5, we investigate if the observed effect on irradiated tumours can be translated as well to tumours outside the RT field. We introduce a new terminology in this chapter, namely the OFRI effect, i.e. the Out-of-Field Radiolmmune effect. We hypothesize that RT and L19-IL2 treatment can induce a curative OFRI effect and can result in long-lasting protection provided by the memory potential of the immune system. We investigated if both single dose and fractionated RT can induce this OFRI effect when combined with L19-IL2. Additionally, we assessed the causal relationship between the OFRI effect and both helper and cytotoxic T cells and whether OFRI is a tumour-specific or is a general effect. For this purpose, secondary tumours were the same (C51) or different (CT26) as compared with the primary tumour (C51). Furthermore, and of great importance, we investigated the potential of RT + L19-IL2 to provide a long-lasting protection against the tumours. We investigated differences to obtain this long-lasting protective effect between RT + L19-IL2, surgery + L19-IL2 and high dose RT + vehicle treatment groups. We additionally assessed if this protection can be predicted using immunological biomarkers (CD44 ${ }^{+} \mathrm{CD} 127^{+}$expression on $\mathrm{CD}^{+} \mathrm{T}$ cells), preferentially in the blood. This might potentially open doors for new biomarker strategies, enabling the classification of long-term responders versus non-responders, creating a possibility to modify treatment for the latter group. In chapter 6, we hypothesize that the combination therapy of RT + L19-IL2 can also trigger a NK dependent anti-tumour response in a model lacking $\mathrm{MHCl}$ expression with the purpose to extend the usefulness of this combination therapy to $\mathrm{MHCl}$ negative tumours or mixed tumours as can be expected in patients. Chapter 7 describes our point-of-view of how to place L19-IL2 in 'the age of the check-point inhibitors' as an answer on an editorial written based on chapter 6 . In chapter 8 we describe the possibility to combat an immunological suppressive mechanisms, the checkpoint CTLA-4 (66) in an attempt for trimodal therapy. In this chapter we have combined the RT (2Gy) + L19-IL2 treatment with anti-CTLA-4 (ipilimumab) and assessed if the therapeutic efficacy of the bimodal combination of RT + L19-IL2 could be improved. Chapter 9 provides a general discussion on all chapters, places our results in a broader context and describes the future perspectives of this exciting research. Inherent to this, chapter 10 describes the way to create impact for patient and society: the valorization. 


\section{REFERENCES}

1. Stewart BW WC. World Cancer Report. IARC. 2014.

2. Couzin-Frankel J. Breakthrough of the year 2013. Cancer immunotherapy. Science. 2013;342 (6165):1432-3.

3. CRI. What Is Cancer Immunotherapy? http://wwwcancerresearchorg/cancer-immunotherapy/what-iscancer-immunotherapy. 2016.

4. Hanahan D, Weinberg RA. The hallmarks of cancer. Cell. 2000;100(1):57-70.

5. Swann JB, Smyth MJ. Immune surveillance of tumors. The Journal of clinical investigation. 2007;117(5):1137-46. PMCID: 1857231.

6. Hanahan D, Weinberg RA. Hallmarks of cancer: the next generation. Cell. 2011;144(5):646-74.

7. van der Burg SH, Arens R, Ossendorp F, van Hall T, Melief CJ. Vaccines for established cancer: overcoming the challenges posed by immune evasion. Nature reviews Cancer. 2016;16(4):219-33.

8. Cheng M, Chen Y, Xiao W, Sun R, Tian Z. NK cell-based immunotherapy for malignant diseases. Cell Mol Immunol. 2013;10(3):230-52. PMCID: 4076738.

9. Murphy K. Janeway's Immunobiology. 2012.

10. Orange JS. Human natural killer cell deficiencies. Curr Opin Allergy Clin Immunol. 2006;6(6):399-409.

11. Morvan MG, Lanier LL. NK cells and cancer: you can teach innate cells new tricks. Nature reviews Cancer. 2016;16(1):7-19.

12. Nakayama Y, Nagashima N, Minagawa N, Inoue Y, Katsuki T, Onitsuka K, et al. Relationships between tumor-associated macrophages and clinicopathological factors in patients with colorectal cancer. Anticancer research. 2002;22(6C):4291-6.

13. Bingle L, Brown NJ, Lewis CE. The role of tumour-associated macrophages in tumour progression: implications for new anticancer therapies. The Journal of pathology. 2002;196(3):254-65.

14. Cheong C, Matos I, Choi JH, Dandamudi DB, Shrestha E, Longhi MP, et al. Microbial stimulation fully differentiates monocytes to DC-SIGN/CD209(+) dendritic cells for immune $T$ cell areas. Cell. 2010;143(3):416-29. PMCID: 3150728.

15. Steinman RM, Idoyaga J. Features of the dendritic cell lineage. Immunological reviews. 2010;234(1):5-17.

16. Schumacher TN, Schreiber RD. Neoantigens in cancer immunotherapy. Science. 2015;348(6230):69-74.

17. Coulie PG, Van den Eynde BJ, van der Bruggen $P$, Boon T. Tumour antigens recognized by $T$ lymphocytes: at the core of cancer immunotherapy. Nature reviews Cancer. 2014;14(2):135-46.

18. Chouaib S, Janji B, Tittarelli A, Eggermont A, Thiery JP. Tumor plasticity interferes with anti-tumor immunity. Crit Rev Immunol. 2014;34(2):91-102.

19. Swartz MA, lida N, Roberts EW, Sangaletti S, Wong MH, Yull FE, et al. Tumor microenvironment complexity: emerging roles in cancer therapy. Cancer research. 2012;72(10):2473-80. PMCID: 3653596.

20. Waldmann TA. The biology of interleukin-2 and interleukin-15: implications for cancer therapy and vaccine design. Nat Rev Immunol. 2006;6(8):595-601.

21. Rosenberg SA. IL-2: the first effective immunotherapy for human cancer. Journal of immunology. 2014;192(12):5451-8.

22. Pardoll DM. The blockade of immune checkpoints in cancer immunotherapy. Nature reviews Cancer. 2012;12(4):252-64. PMCID: 4856023.

23. Fridman WH, Pages F, Sautes-Fridman C, Galon J. The immune contexture in human tumours: impact on clinical outcome. Nature reviews Cancer. 2012;12(4):298-306.

24. Morgan RA, Dudley ME, Wunderlich JR, Hughes MS, Yang JC, Sherry RM, et al. Cancer regression in patients after transfer of genetically engineered lymphocytes. Science. 2006;314(5796):126-9. PMCID: 2267026.

25. Robbins PF, Morgan RA, Feldman SA, Yang JC, Sherry RM, Dudley ME, et al. Tumor regression in patients with metastatic synovial cell sarcoma and melanoma using genetically engineered lymphocytes reactive with NY-ESO-1. Journal of clinical oncology : official journal of the American Society of Clinical Oncology. 2011;29(7):917-24. PMCID: 3068063. 
26. Wolchok JD, Kluger H, Callahan MK, Postow MA, Rizvi NA, Lesokhin AM, et al. Nivolumab plus ipilimumab in advanced melanoma. The New England journal of medicine. 2013;369(2):122-33.

27. Yang JC, Haworth L, Sherry RM, Hwu P, Schwartzentruber DJ, Topalian SL, et al. A randomized trial of bevacizumab, an anti-vascular endothelial growth factor antibody, for metastatic renal cancer. The New England journal of medicine. 2003;349(5):427-34. PMCID: 2275324.

28. Sim GC, Radvanyi L. The IL-2 cytokine family in cancer immunotherapy. Cytokine Growth Factor Rev. 2014;25(4):377-90.

29. Assier E, Jullien V, Lefort J, Moreau JL, Vargaftig BB, Lapa e Silva JR, et al. Constitutive expression of IL2Rbeta chain and its effects on IL-2-induced vascular leak syndrome. Cytokine. 2005;32(6):280-6.

30. Gately MK, Anderson TD, Hayes TJ. Role of asialo-GM1-positive lymphoid cells in mediating the toxic effects of recombinant IL-2 in mice. Journal of immunology. 1988;141(1):189-200.

31. Peace DJ, Cheever MA. Toxicity and therapeutic efficacy of high-dose interleukin 2. In vivo infusion of antibody to NK-1.1 attenuates toxicity without compromising efficacy against murine leukemia. The Journal of experimental medicine. 1989;169(1):161-73. PMCID: 2189181.

32. Neri D, Sondel PM. Immunocytokines for cancer treatment: past, present and future. Current opinion in immunology. 2016;40:96-102.

33. Carnemolla B, Borsi L, Balza E, Castellani P, Meazza R, Berndt A, et al. Enhancement of the antitumor properties of interleukin-2 by its targeted delivery to the tumor blood vessel extracellular matrix. Blood. 2002;99(5):1659-65.

34. Balza E, Carnemolla B, Mortara L, Castellani P, Soncini D, Accolla RS, et al. Therapy-induced antitumor vaccination in neuroblastomas by the combined targeting of $\mathrm{IL}-2$ and TNFalpha. Int J Cancer. 2010;127(1):101-10.

35. Rossin R, Berndorff D, Friebe M, Dinkelborg LM, Welch MJ. Small-animal PET of tumor angiogenesis using a (76)Br-labeled human recombinant antibody fragment to the ED-B domain of fibronectin. Journal of nuclear medicine : official publication, Society of Nuclear Medicine. 2007;48(7):1172-9.

36. Tijink BM, Neri D, Leemans CR, Budde M, Dinkelborg LM, Stigter-van Walsum $M$, et al. Radioimmunotherapy of head and neck cancer xenografts using 1311-labeled antibody L19-SIP for selective targeting of tumor vasculature. J Nucl Med. 2006;47(7):1127-35.

37. Eigentler TK, Weide B, de Braud F, Spitaleri G, Romanini A, Pflugfelder A, et al. A dose-escalation and signal-generating study of the immunocytokine L19-IL2 in combination with dacarbazine for the therapy of patients with metastatic melanoma. Clin Cancer Res. 2011;17(24):7732-42.

38. Johannsen M, Spitaleri G, Curigliano G, Roigas J, Weikert S, Kempkensteffen C, et al. The tumourtargeting human L19-IL2 immunocytokine: preclinical safety studies, phase I clinical trial in patients with solid tumours and expansion into patients with advanced renal cell carcinoma. Eur J Cancer. 2010;46(16):2926-35.

39. Leavy O. Tumour immunology: A triple blow for cancer. Nat Rev Immunol. 2015;15(5):265.

40. Twyman-Saint Victor C, Rech AJ, Maity A, Rengan R, Pauken KE, Stelekati E, et al. Radiation and dual checkpoint blockade activate non-redundant immune mechanisms in cancer. Nature. 2015;520(7547):373-7. PMCID: 4401634.

41. Delaney G, Jacob S, Featherstone C, Barton M. The role of radiotherapy in cancer treatment: estimating optimal utilization from a review of evidence-based clinical guidelines. Cancer. 2005;104(6):1129-37.

42. Prise KM, Schettino G, Folkard M, Held KD. New insights on cell death from radiation exposure. The Lancet Oncology. 2005;6(7):520-8.

43. Obeid M, Tesniere A, Ghiringhelli F, Fimia GM, Apetoh L, Perfettini JL, et al. Calreticulin exposure dictates the immunogenicity of cancer cell death. Nature medicine. 2007;13(1):54-61.

44. Apetoh L, Ghiringhelli F, Tesniere A, Obeid M, Ortiz C, Criollo A, et al. Toll-like receptor 4-dependent contribution of the immune system to anticancer chemotherapy and radiotherapy. Nature medicine. 2007;13(9):1050-9.

45. Ghiringhelli F, Apetoh L, Tesniere A, Aymeric L, Ma Y, Ortiz C, et al. Activation of the NLRP3 inflammasome in dendritic cells induces IL-1beta-dependent adaptive immunity against tumors. Nature medicine. 2009;15(10):1170-8. 
46. McBride WH, Chiang CS, Olson JL, Wang CC, Hong JH, Pajonk F, et al. A sense of danger from radiation. Radiat Res. 2004;162(1):1-19.

47. Golden EB, Apetoh L. Radiotherapy and immunogenic cell death. Seminars in radiation oncology. 2015;25(1):11-7.

48. Barker HE, Paget JT, Khan AA, Harrington KJ. The tumour microenvironment after radiotherapy: mechanisms of resistance and recurrence. Nature reviews Cancer. 2015;15(7):409-25. PMCID: 4896389.

49. Larson SM, Carrasquillo JA, Cheung NK, Press OW. Radioimmunotherapy of human tumours. Nature reviews Cancer. 2015;15(6):347-60. PMCID: 4798425.

50. Demaria M, Ohtani N, Youssef SA, Rodier F, Toussaint W, Mitchell JR, et al. An essential role for senescent cells in optimal wound healing through secretion of PDGF-AA. Developmental cell. 2014;31(6):722-33. PMCID: 4349629.

51. Demaria S, Ng B, Devitt ML, Babb JS, Kawashima N, Liebes L, et al. Ionizing radiation inhibition of distant untreated tumors (abscopal effect) is immune mediated. International journal of radiation oncology, biology, physics. 2004;58(3):862-70.

52. Okuma K, Yamashita H, Niibe Y, Hayakawa K, Nakagawa K. Abscopal effect of radiation on lung metastases of hepatocellular carcinoma: a case report. J Med Case Rep. 2011;5:111. PMCID: 3069951.

53. Wersall PJ, Blomgren H, Pisa P, Lax I, Kalkner KM, Svedman C. Regression of non-irradiated metastases after extracranial stereotactic radiotherapy in metastatic renal cell carcinoma. Acta Oncol. 2006;45(4):493-7.

54. Mole RH. Whole body irradiation; radiobiology or medicine? Br J Radiol. 1953;26(305):234-41.

55. Grass GD, Krishna N, Kim S. The immune mechanisms of abscopal effect in radiation therapy. Curr Probl Cancer. 2016;40(1):10-24.

56. Ehlers G, Fridman M. Abscopal effect of radiation in papillary adenocarcinoma. Br J Radiol. 1973;46(543):220-2.

57. Kingsley DP. An interesting case of possible abscopal effect in malignant melanoma. Br J Radiol. 1975;48(574):863-6.

58. Nobler MP. The abscopal effect in malignant lymphoma and its relationship to lymphocyte circulation. Radiology. 1969;93(2):410-2.

59. Ohba K, Omagari K, Nakamura T, Ikuno N, Saeki S, Matsuo I, et al. Abscopal regression of hepatocellular carcinoma after radiotherapy for bone metastasis. Gut. 1998;43(4):575-7. PMCID: 1727260.

60. Rees GJ. Abscopal regression in lymphoma: a mechanism in common with total body irradiation? Clin Radiol. 1981;32(4):475-80.

61. Sham RL. The abscopal effect and chronic lymphocytic leukemia. Am J Med. 1995;98(3):307-8.

62. Vatner RE, Cooper BT, Vanpouille-Box C, Demaria S, Formenti SC. Combinations of immunotherapy and radiation in cancer therapy. Frontiers in oncology. 2014;4:325. PMCID: 4246656.

63. Clarke M, Collins R, Darby S, Davies C, Elphinstone P, Evans V, et al. Effects of radiotherapy and of differences in the extent of surgery for early breast cancer on local recurrence and 15-year survival: an overview of the randomised trials. Lancet. 2005;366(9503):2087-106.

64. Darby S, McGale P, Correa C, Taylor C, Arriagada R, Clarke M, et al. Effect of radiotherapy after breastconserving surgery on 10-year recurrence and 15-year breast cancer death: meta-analysis of individual patient data for 10,801 women in 17 randomised trials. Lancet. 2011;378(9804):1707-16. PMCID: 3254252.

65. Formenti SC, Demaria S. Systemic effects of local radiotherapy. Lancet Oncol. 2009;10(7):718-26. PMCID: 2782943.

66. Lenschow DJ, Walunas TL, Bluestone JA. CD28/B7 system of T cell costimulation. Annual review of immunology. 1996;14:233-58.

67. Dranoff G. Cytokines in cancer pathogenesis and cancer therapy. Nature reviews. Cancer 2004;4:11-22. 
Chapter

\section{Stereotactic ablative body Radiotherapy combined with immunotherapy: present status and future perspectives}

$\underline{\text { N.H. Rekers }}{ }^{*}$, E.G.C. Troost ${ }^{*}$, C.M.L. Zegers, W.T.V. Germeraad, L. Dubois ${ }^{* *}$, P. Lambin ${ }^{* *}$

Published in: Cancer Radiother. 2014; 18(5-6):391-5 


\section{ABSTRACT}

Radiotherapy is along with surgery and chemotherapy one of the prime treatment modalities in cancer. It is applied in the primary, neoadjuvant as well as the adjuvant setting. Radiation techniques have rapidly evolved during the past decade enabling the delivery of high radiation doses, reducing side-effects in tumour-adjacent normal tissues. While increasing local tumour control, current and future efforts ought to deal with microscopic disease at a distance of the primary tumour, ultimately responsible for disease progression. This review explores the possibility of bi-modal treatment combining radiotherapy with immunotherapy. 


\section{STEREOTACTIC ABLATIVE BODY RADIOTHERAPY}

Stereotactic ablative body radiotherapy (SABR) is a form of high-precision radiotherapy delivering extremely high ablative doses of radiation, usually in 3-8 fractions, combining reproducible patient immobilization, tumour motion tracking and steep dose gradients, resulting in reduced normal tissue toxicity (1). SABR achieves excellent local control rates in patients with stage I/II non-small cell lung cancer (NSCLC) and liver metastases of colorectal cancer (CRC) (2). Nowadays, these favorable results of SABR are being transferred to patients with limited sites of metastatic disease (oligometastatic; $\leq 5$ metastases in $\leq 3$ organs) originating from solid tumours (e.g., breast, NSCLC, head and neck, renal cell carcinoma, melanoma, CRC), both at primary diagnosis (synchronous) and during the course of disease (3-10). Tree et al. reports on favorable local control rates of approximately 80\% using SABR with few treatment-related side-effects (10). Recently, our group (11) found NSCLC patients with synchronous oligometastases to have a median progression-free survival (PFS) of 12.1 months when treated radically to all known metastatic sites. However, in the vast majority of patients, diseaseprogression at distance from the treated site occurs ultimately leading to extensive metastatic disease and cancer-related death.

\section{TUMOURIGENESIS AND THE IMMUNE SYSTEM}

The immune system closely monitors the process of tumourigenesis first by registering the presence of cells undergoing neoplastic transformation, and second by interacting with neoplastic cells to mediate their destruction. Solid tumours have developed mechanisms to escape "cancer immunosurveillance", i.e., detection by the immune system. This is achieved by, among other mechanisms, the secretion of potent immunesuppressive cytokines and the expression of T-cell inhibitory molecules, which are able to down-regulate an anti-tumour immune response (12). There is conclusive evidence that, apart from its direct effects, RT can induce "immunogenic cell death", which serves as a trigger or "in situ vaccine" for the innate and adaptive immune system (1315). RT induces immunogenic cell death by the release of tumour antigens and damage associated molecular patterns (DAMPS), including high-mobility group protein B1 (HMGB1), adenosine triphosphate (ATP) and the exposure of calreticulin on the tumour cell surface. Also, several cellular surface expression molecules, including Fas and ICAM1 , are upregulated $(16,17)$. These factors promote uptake of dying cells by dendritic cells, cross-presentation of tumour antigens to $T$ cells, and activation of anti-tumour (cytotoxic) T cells $(18,19)$.

Recent preclinical and clinical data indicate that immunogenic cell death may be an important consequence of ionizing radiation (18), and that localized radiotherapy can 
evoke and/or modulate tumour-associated immune responses (20). Even though clinical evidence of systemic anti-tumour response from local irradiation is scarce, tumour regression outside the irradiated field was already recognized in 1953 and termed abscopal effect (21-25). In general, it is unlikely that radiotherapy alone provides a sufficient anti-tumour immune response and the addition of active immunotherapy (IT) to SABR may increase the therapeutic potential and induce abscopal effects in a more systematic way $(24,26)$.

\section{RECENT CLINICAL SUCCESSES USING IMMUNOTHERAPY}

\section{Breaking the immune tolerance using checkpoint modulators}

Immune checkpoints refer to a plethora of inhibitory pathways hardwired into the immune system. These are crucial for maintaining self-tolerance and modulating the duration and amplitude of physiological immune responses in peripheral tissues, in order to minimize collateral tissue damage. It is now clear that tumours use certain immune-checkpoint pathways as a major mechanism of immune resistance, particularly against tumour antigen specific T-cells. Examples of these immune-checkpoints are the cytotoxic $T$ lymphocyte-associated antigen 4 (CTLA4), or the programmed death receptor 1 (PD-1) and its ligand PD-L1. New strategies aim at breaking this tolerance. Monoclonal antibody-mediated (ipilimumab) blockade of CTLA-4 on T cells seems to be sufficient to elicit an effective anti-tumour immunity (27), which paved the way for clinical studies. Two phase III studies evaluated the clinical effects of ipilimumab in metastatic melanoma patients. Treatment with ipilimumab as monotherapy improved median overall survival rates from 6.4 to 10 months (28), and bi-modal treatment with standard of care chemotherapy (dacarbazine) increased 3-year overall survival from $12.2 \%$ to $20.8 \%$ (29). Although the percentage of patients responding to ipilimumab was limited (complete response $\sim 1 \%$, partial response in $5-10 \%$ ), the effects of response were long-lasting in those who responded. Even though treatment-related adverse effects occurred in almost all patients, with several immune effects-related deaths in the first trial, and despite the high costs, ipilimumab received FDA approval in 2011 for treatment of advanced melanoma patients due to its clear clinical effect.

\section{Immunotherapy for other solid tumours}

The effect of immunotherapy has increasingly been evaluated in both immunogenic and non-immunogenic (metastatic) solid tumours, including prostate cancer, renal cell carcinoma, melanoma, and head and neck cancer. In the subsequent paragraph the diversity and recent merits of this approach are highlighted. The autologous active cellular immunotherapy, Sipuleucel-T, significantly reduced the risk of death in 
metastatic castration-resistant prostate cancer (mCRPC) patients compared to the placebo group (30). Also, prostate-specific antigen (PSA)-targeted poxviral vaccines were well-tolerated and associated with an 8.5-month improvement in median overall survival for MCRPC patients (31). Recent advances for MCRPC were well summarized by Flemming (32). Rini et al. (33) randomized patients with metastastic RCC into two cohorts, one receiving bevacizumab plus interferon alpha (IFN- $\alpha$ ), the other IFN- $\alpha$ only. The combination treatment led to a slightly prolonged overall survival time but did not meet expectations, i.e., it was impossible to identify patient subgroups benefitting from the combined treatment. Autologous cytokine-induced killer cell immunotherapy was found to be superior to combined IL2 and IFN $\alpha$ treatment in terms of 3-year PFS and OS in metastatic RCC patient (34). For head and neck cancer (HNSCC) patients, research efforts include the development of the vaccine DRibble, stimulating tumour-infiltrating T-lymphocytes (35) and immunotherapy of Human Papilloma Virus (HPV)-associated HNSCC (36) amongst others.

\section{SABR COMBINED WITH IMMUNOTHERAPY}

\section{Preclinical results}

Several studies have focused on the immunogenic response of tumours to different dose schedules of radiotherapy. Lee et al. (37) observed that a single dose of ablative radiotherapy ( $R T$; $20 \mathrm{~Gy}$ ) generated a $\mathrm{CD}^{+} \mathrm{T}$ cell-dependent immunity leading to tumour reduction and eradication of metastasis. In comparison, mice treated with $4 \times 5$ Gy initially responded to RT but tumours relapsed over time. One possible explanation is that fractionated low-dose RT may kill infiltrating CD8+ T cells over time. However, when ablative RT ( 2 × 12 Gy) was combined with ad-LIGHT-based immunotherapy, circulating cytotoxic $T$ cells increased again and micrometastases were eradicated (37). Lugade et al. (38) observed activated and expanded anti-tumour CD8+ T cells in response to 5 fractions of $3 \mathrm{~Gy}$, however without resulting in tumour growth delay. Although these studies indicate that ablative RT is able to provoke a CD8+ T cell mediated immune response, most studies only detected an immune response after RT combined with different immunotherapies.

Several in vivo studies have investigated the combination of radiotherapy with antiCTLA-4 based immunotherapy. In a metastasizing breast cancer mouse model, a single dose (12 Gy) of radiotherapy to the primary tumour combined with systemic anti-CTLA4 blocking antibody $9 \mathrm{H} 10$ immunotherapy decreased metastatic burden, but the effect on the primary tumours was minimal. Two RT fractions of 12 Gy delivered to the primary tumour in combination with CTLA-4 blockade resulted in complete regression of the irradiated tumour and metastases in the majority of the mice, a response which 
was CD8+ T cell mediated (39). Dewan et al. (40) tested different radiation regimens similar to SABR ( $1 \times 20$ Gy, $3 \times 8$ Gy or $5 \times 6$ Gy) in combination with anti-CTLA-4 in two poorly immunogenic tumour models and observed a growth delay of the irradiated tumour in all treatment schedules. However, an anti-tumour effect outside the radiation field (abscopal effect) was only observed for the combination of anti-CTLA-4 with fractionated RT (3 x 8 Gy). Furthermore, Verbrugge et al. (41) investigated a combination of antibodies against the immune modulators CD137 and PD-1, in combination with single dose (12 Gy) or fractionated ( $4 \times 4$ or 5 Gy) radiotherapy in an orthotropic mice model. Antibody combination therapy with single dose RT was effective and the combination with either $4 \times 4$ Gy or $4 \times 5$ Gy showed tumour rejection rates of $40 \%$ and $80 \%$, respectively. Furthermore, combination of single dose (12 Gy) RT with anti-PD-L1 treatment activated a cytotoxic $T$ cell response, which resulted in tumour regression. An additional explanation for the local tumour control is the reduction of local accumulation of myeloid-derived suppressor cells (42). Additionally, RT has been shown to reduce tumour growth within and outside the radiation field when combined with the DC growth factor Flt3-L (43), the human macrophage inflammatory protein-1 alpha variant (ECI301) (44) or with IL-2 administration $(45,46)$. Experiments with syngeneic tumour-bearing mice showed that blocking AnnexinA5 resulted in decreased DC clearance by macrophages, improved tumour immunogenicity, and combination with RT led to an effective tumour growth inhibition (47). Recently, RT combined with adenoviral-mediated vaccination against the colorectal cancer antigen GUCY2C resulted in a specific $T$ cell response leading to tumour eradication (48).

\section{Clinical results}

There are limited clinical results on the combination of SABR with immunotherapy. Postow et al. (24) were the first to observe an anti-tumour immunologic rejection of a metastatic lesion at distance from the irradiated site and more reports followed (49-51). In parallel, several phase II/II clinical trials have been conducted leading to recent publications. The phase $\mathrm{I} / \mathrm{II}$ study in patients with $\mathrm{MCRPC}$ found ipilimumab \pm radiotherapy to induce anti-tumour activity with disease control and manageable sideeffects (52). However, Kwon et al. (53) randomized between ipilimumab or placebo after radiotherapy in $\mathrm{MCRPC}$ patients and found no difference in terms of overall survival. Several clinical studies on combining immunotherapy (ipilimumab, PD-1 and PD-L1) with (stereotactic ablative body) radiotherapy in diverse solid tumours (i.e., melanoma, CRC, HNSCC, cervical cancer, (non-) small cell lung cancer, prostate and pancreatic cancer) are currently being conducted and results are awaited eagerly (www.clinicaltrials.gov). 


\section{CONCLUSION}

In conclusion, these data show that immunogenic cell death caused by different strategies of RT can be used in combination with immunotherapy to induce a CD8+ T cell mediated anti-tumour response, which leads to tumour control of the irradiated tumour and often to tumour control outside the radiation field, i.e., an abscopal effect in different preclinical models. However, there is not yet a uniform combination strategy for the best RT schedule/dose and immunotherapeutic approach. Furthermore, these preclinical studies often show no effect when immunotherapy is used without RT, suggesting that RT plays a key role as immunogenic trigger, which can be further enhanced when boosting the immune system. Clinical studies are focusing on different immunotherapies, often trying to activate or prolong specific anti-tumour T-cell responses, showing promising responses. However, the administration of immunotherapy adjuvantly to RT thus activating or prolonging T-cell responses specific to the irradiated tumour may increase the immune response inside the radiation field and at metastatic sides. Therefore, it may be important to start with a trigger received from SABR before administrating immunotherapy, because of the 'priming' role of RT in this anti-tumour process.

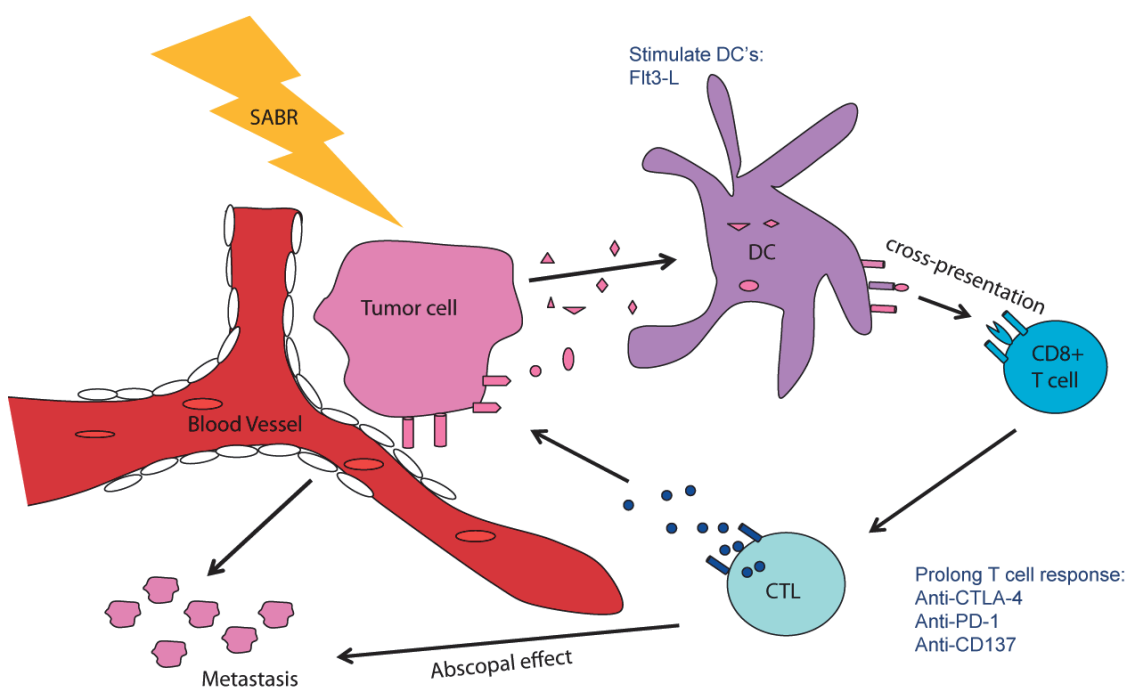

Immunogenic cell death by SABR. SABR can induce immunogenic cell death by the release of tumour antigens and damage associated molecular patterns (DAMPS), including high-mobility group protein B1 (HMGB1), adenosine triphosphate (ATP), Fas, ICAM-1 and the exposure of calreticulin on the tumour cell surface. These factors promote uptake of dying cells by dendritic cells, cross-presentation of antigens to CD8+ T cells and activation of cytotoxic T cells. However, it is unlikely that radiotherapy alone provides a sufficient anti-tumour immune response. The addition of immunotherapy that stimulate DC's, such as Flt3-L or that activate and prolong $T$ cell responses, including anti-CTLA-4, anti-PD-1 or anti-CD137 can be combined with SABR to increase therapeutic potential and abscopal effects in a more systematic way. 


\section{REFERENCES}

1. Jaffray, D.A., Image-guided radiotherapy: from current concept to future perspectives. Nature reviews. Clinical oncology, 2012. 9(12): p. 688-99.

2. van Baardwijk, A., et al., Is high-dose stereotactic body radiotherapy (SBRT) for stage I non-small cell lung cancer (NSCLC) overkill? A systematic review. Radiotherapy and oncology : journal of the European Society for Therapeutic Radiology and Oncology, 2012. 105(2): p. 145-9.

3. Ashworth, A., et al., Is there an oligometastatic state in non-small cell lung cancer? A systematic review of the literature. Lung Cancer, 2013. 82(2): p. 197-203.

4. Cheruvu, P., et al., Comparison of outcomes in patients with stage III versus limited stage IV non-small cell lung cancer. Radiation oncology, 2011. 6: p. 80.

5. Dahele, M. and S. Senan, The role of stereotactic ablative radiotherapy for early-stage and oligometastatic non-small cell lung cancer: evidence for changing paradigms. Cancer research and treatment : official journal of Korean Cancer Association, 2011. 43(2): p. 75-82.

6. Hasselle, M.D., et al., Hypofractionated image-guided radiation therapy for patients with limited volume metastatic non-small cell lung cancer. J Thorac Oncol, 2012. 7(2): p. 376-81.

7. Higginson, D.S., et al., The impact of local and regional disease extent on overall survival in patients with advanced stage IIIB/IV non-small cell lung carcinoma. Int J Radiat Oncol Biol Phys, 2012. 84(3): p. e385-92.

8. Marks, L.B., M. Saynak, and J.P. Christodouleas, Stage III vs. stage IV lung cancer: "Crossing a Great Divide". Lung Cancer, 2010. 67(1): p. 1-3.

9. Milano, M.T., et al., Oligometastases treated with stereotactic body radiotherapy: long-term follow-up of prospective study. Int J Radiat Oncol Biol Phys, 2012. 83(3): p. 878-86.

10. Tree, A.C., et al., Stereotactic body radiotherapy for oligometastases. The lancet oncology, 2013. 14(1): p. e28-37.

11. De Ruysscher, D., et al., Radical treatment of non-small-cell lung cancer patients with synchronous oligometastases: long-term results of a prospective phase II trial (Nct01282450). J Thorac Oncol, 2012. 7(10): p. 1547-55.

12. Keast, D., Immunosurveillance and cancer. Lancet, 1970. 2(7675): p. 710-2.

13. Demaria, S., et al., Combining radiotherapy and immunotherapy: a revived partnership. International journal of radiation oncology, biology, physics, 2005. 63(3): p. 655-66.

14. Formenti, S.C. and S. Demaria, Radiation therapy to convert the tumor into an in situ vaccine. Int J Radiat Oncol Biol Phys, 2012. 84(4): p. 879-80.

15. McBride, W.H., et al., A sense of danger from radiation. Radiation research, 2004. 162(1): p. 1-19.

16. Chakraborty, M., et al., Irradiation of tumor cells up-regulates Fas and enhances CTL lytic activity and CTL adoptive immunotherapy. Journal of immunology, 2003. 170(12): p. 6338-47.

17. Garnett, C.T., et al., Sublethal irradiation of human tumor cells modulates phenotype resulting in enhanced killing by cytotoxic T lymphocytes. Cancer research, 2004. 64(21): p. 7985-94.

18. Apetoh, L., et al., The interaction between HMGB1 and TLR4 dictates the outcome of anticancer chemotherapy and radiotherapy. Immunol Rev, 2007. 220: p. 47-59.

19. Obeid, M., et al., Calreticulin exposure dictates the immunogenicity of cancer cell death. Nature medicine, 2007. 13(1): p. 54-61.

20. Reits, E.A., et al., Radiation modulates the peptide repertoire, enhances MHC class I expression, and induces successful antitumor immunotherapy. J Exp Med, 2006. 203(5): p. 1259-71.

21. Kroemer, G. and L. Zitvogel, Abscopal but desirable: The contribution of immune responses to the efficacy of radiotherapy. Oncoimmunology, 2012. 1(4): p. 407-408.

22. Masucci, G.V., et al., Stereotactic Ablative Radio Therapy (SABR) followed by immunotherapy a challenge for individualized treatment of metastatic solid tumours. J Transl Med, 2012. 10: p. 104.

23. Mole, R.H., Whole body irradiation; radiobiology or medicine? Br J Radiol, 1953. 26: p. 234-241.

24. Postow, M.A., et al., Immunologic correlates of the abscopal effect in a patient with melanoma. N Engl J Med, 2012. 366(10): p. 925-31. 
25. Stamell, E.F., et al., The Abscopal Effect Associated With a Systemic Anti-melanoma Immune Response. Int J Radiat Oncol Biol Phys, 2012.

26. Kachikwu, E.L., et al., Radiation enhances regulatory $T$ cell representation. International journal of radiation oncology, biology, physics, 2011. 81(4): p. 1128-35.

27. Kwon, E.D., et al., Manipulation of $T$ cell costimulatory and inhibitory signals for immunotherapy of prostate cancer. Proc Natl Acad Sci U S A, 1997. 94(15): p. 8099-103.

28. Hodi, F.S., et al., Improved survival with ipilimumab in patients with metastatic melanoma. N Engl J Med, 2010. 363(8): p. 711-23.

29. Robert, C., et al., Ipilimumab plus dacarbazine for previously untreated metastatic melanoma. N Engl J Med, 2011. 364(26): p. 2517-26.

30. Kantoff, P.W., et al., Sipuleucel-T immunotherapy for castration-resistant prostate cancer. The New England journal of medicine, 2010. 363(5): p. 411-22.

31. Kantoff, P.W., et al., Overall survival analysis of a phase II randomized controlled trial of a Poxviral-based PSA-targeted immunotherapy in metastatic castration-resistant prostate cancer. Journal of clinical oncology : official journal of the American Society of Clinical Oncology, 2010. 28(7): p. 1099-105.

32. Flemming, A., Immunotherapy: A vaccine for prostate cancer? Nature reviews. Cancer, 2011. 11(8): p. 539.

33. Rini, B.I., et al., Phase III trial of bevacizumab plus interferon alfa versus interferon alfa monotherapy in patients with metastatic renal cell carcinoma: final results of CALGB 90206. Journal of clinical oncology: official journal of the American Society of Clinical Oncology, 2010. 28(13): p. 2137-43.

34. Liu, L., et al., Randomized study of autologous cytokine-induced killer cell immunotherapy in metastatic renal carcinoma. Clinical cancer research : an official journal of the American Association for Cancer Research, 2012. 18(6): p. 1751-9.

35. Moudgil, T., et al., Developing an immunotherapy strategy for the effective treatment of patients with squamous cell carcinoma of the head and neck. Journal for ImmunoTherapy 2013. 1(Suppl 1)p. P262 doi:10.1186/2051-1426-1-S1-P262.

36. Nizard, M., et al., Immunotherapy of HPV-associated head and neck cancer: Critical parameters. Oncoimmunology, 2013. 2(6): p. e24534.

37. Lee, Y., et al., Therapeutic effects of ablative radiation on local tumor require CD8+ $T$ cells: changing strategies for cancer treatment. Blood, 2009. 114(3): p. 589-95.

38. Lugade, A.A., et al., Local radiation therapy of B16 melanoma tumors increases the generation of tumor antigen-specific effector cells that traffic to the tumor. Journal of immunology, 2005. 174(12): p. 7516-23.

39. Demaria, S., et al., Immune-mediated inhibition of metastases after treatment with local radiation and CTLA-4 blockade in a mouse model of breast cancer. Clin Cancer Res, 2005. 11(2 Pt 1): p. 728-34.

40. Dewan, M.Z., et al., Fractionated but not single-dose radiotherapy induces an immune-mediated abscopal effect when combined with anti-CTLA-4 antibody. Clin Cancer Res, 2009. 15(17): p. 5379-88.

41. Verbrugge, I., et al., Radiotherapy increases the permissiveness of established mammary tumors to rejection by immunomodulatory antibodies. Cancer research, 2012. 72(13): p. 3163-74.

42. Deng, L., et al., Irradiation and anti-PD-L1 treatment synergistically promote antitumor immunity in mice. The Journal of clinical investigation, 2014. 124(2): p. 687-95.

43. Demaria, S., et al., lonizing radiation inhibition of distant untreated tumors (abscopal effect) is immune mediated. International journal of radiation oncology, biology, physics, 2004. 58(3): p. 862-70.

44. Shiraishi, K., et al., Enhancement of antitumor radiation efficacy and consistent induction of the abscopal effect in mice by ECI301, an active variant of macrophage inflammatory protein-1alpha. Clinical cancer research : an official journal of the American Association for Cancer Research, 2008. 14(4): p. 1159-66.

45. Everse, L.A., et al., Priming of the antitumor response promotes efficacy of interleukin-2 therapy. Cancer immunology, immunotherapy : CII, 1997. 44(4): p. 221-9.

46. Jurgenliemk-Schulz, I.M., et al., Anti-tumor effects of local irradiation in combination with peritumoral administration of low doses of recombinant interleukin-2 (rIL-2). Radiation oncology investigations, 1997. 5(2): p. 54-61.

47. Frey, B., et al., AnnexinA5 renders dead tumor cells immunogenic--implications for multimodal cancer therapies. Journal of immunotoxicology, 2009. 6(4): p. 209-16. 
48. Witek, M., et al., Tumor radiation therapy creates therapeutic vaccine responses to the colorectal cancer antigen GUCY2C. International journal of radiation oncology, biology, physics, 2014. 88(5): p. 1188-95.

49. Golden, E.B., et al., An abscopal response to radiation and ipilimumab in a patient with metastatic nonsmall cell lung cancer. Cancer immunology research, 2013. 1(6): p. 365-72.

50. Hiniker, S.M., D.S. Chen, and S.J. Knox, Abscopal effect in a patient with melanoma. The New England journal of medicine, 2012. 366(21): p. 2035; author reply 2035-6.

51. Stamell, E.F., et al., The abscopal effect associated with a systemic anti-melanoma immune response. International journal of radiation oncology, biology, physics, 2013. 85(2): p. 293-5.

52. Slovin, S.F., et al., Ipilimumab alone or in combination with radiotherapy in metastatic castrationresistant prostate cancer: results from an open-label, multicenter phase I/II study. Annals of oncology: official journal of the European Society for Medical Oncology / ESMO, 2013. 24(7): p. 1813-21.

53. Kwon, E.D., et al., Ipilimumab versus placebo after radiotherapy in patients with metastatic castrationresistant prostate cancer that had progressed after docetaxel chemotherapy (CA184-043): a multicentre, randomised, double-blind, phase 3 trial. The lancet oncology, 2014. 
Chapter

\section{Radiotherapy combined with the immunocytokine L19-IL2 provides long-lasting anti-tumour effects}

Catharina M.L. Zegers*, Nicolle H. Rekers*, Dana H.F. Quaden, Natasja G. Lieuwes, Ala Yaromina, Wilfred T.V. Germeraad, Lotte Wieten, Erik A.L. Biessen, Louis Boon, Dario Neri, Esther G.C. Troost, Ludwig J. Dubois**, and Philippe Lambin**

Published in: Clin Cancer Res. 2015;21(5):1151-60 


\section{ABSTRACT}

Radiotherapy (RT) causes the release of tumour antigens activating the immune system, which can be enhanced by interleukin-2 (IL2) immunotherapy. L19 targets the extra domain B (ED-B) of fibronectin, a marker for tumour neo-angiogenesis, and can be used as immunocytokine when fused to IL2 (L19-IL2). We hypothesized that RT in combination with L19-IL2 provides an enhanced anti-tumour effect, dependent on ED-B expression. In mice bearing syngeneic C51 colon carcinoma, Lewis lung carcinoma (LLC) or 4T1 mammary carcinoma, having high, intermediate or low ED-B expression, respectively, tumour growth delay and immunological mechanisms were evaluated after local tumour irradiation combined with systemic administration of L19-IL2 or equimolar controls. The combination therapy (RT+L19-IL2) showed a long-lasting synergistic effect for the C51 model with 9 of 12 tumours cured, an additive effect for the LLC model and no additional effect for the 4T1 model. Depletion of CD8 ${ }^{+} \mathrm{T}$ cells in consecutive experiments prevented the beneficial effects of RT+L19-IL2 co-therapy. To our knowledge, these data provide the first evidence for an increased therapeutic potential by combining RT with L19-IL2 and legitimates further evaluation in phase-I clinical studies. 


\section{INTRODUCTION}

Radiotherapy (RT) causes cell cycle arrest or programmed cell death in rapidly proliferating cancer cells through the induction of DNA damage. Irradiated tumours stimulate the immune system by releasing tumour antigens, damage associated molecular patterns (DAMPs), and an upregulation of immunomodulatory cell surface and secretory molecules (1-3). This promotes the uptake of dying cells by antigen presenting cells, and provides cross-presentation of the tumour-derived antigens to T cells, thereby triggering a cytotoxic T-lymphocyte response, which might cause immunogenic cell death (ICD) $(1,4,5)$. In some cases, tumour growth inhibition outside the field of radiation is observed, termed abscopal effect, which suggests the presence of a systemic radiationinduced anti-tumour immune response (6-9). However, in general, it is unlikely that radiotherapy alone provides a sufficient anti-tumour immune response. Therefore, the addition of active immunotherapy (IT) may increase the therapeutic potential (10-12).

Active immunotherapy is used to stimulate the immune system acting against tumour cells. Cytotoxic T-lymphocytes and Natural Killer (NK) cells play an important complementary role in the anti-tumour immune response since they release specialized Iytic granules, which upon interaction with the tumour cell create pores in the lipid bilayer of the target cell resulting in cell death $(13,14)$. Interleukin-2 (IL2) is a cytokine with an essential role in the activation phase of the immune response; it stimulates the proliferation of cytotoxic T cells, NK cells and regulatory T cells, providing a balance between a pro- and anti-inflammatory immune response $(15,16)$. Systemic administration of IL2 was introduced as immunotherapy for patients with metastatic melanoma and renal cell carcinoma, which resulted in a higher tumour response and survival (17). However, to reach an effective intra-tumoural dose of IL2 by systemic administration, high doses ought to be administered, which often leads to toxicity (e.g. capillary leakage syndrome, severe flu-like symptoms, and coma) (18). Currently, the use of intra-tumoural injections of IL2 are investigated to reach a higher local concentration of IL2 $(19,20)$, which shows promising results in combination with RT in a preclinical setting (21), however these intra-tumoural injections are limited to accessible lesions.

An interesting alternative is the selective delivery of IL2 to the tumour by use of fusion proteins $(15,22)$. During tumour progression, synthesis of extracellular matrix components occurs, with in particular a modulation of vascular cell behaviour and angiogenesis (15). Fibronectin of the tumour neovasculature expresses extradomain- $B$ (ED-B), which is preserved in mouse, human and other mammals. ED-B expression can be used for targeted therapies because it is over-expressed in various solid tumours (e.g. melanoma, RCC, breast, colorectal, and non-small cell lung cancer), but absent in plasma and normal tissue fibronectin (except for regenerating tissues) (23-28). The small-immuno-protein (SIP) L19 was developed to specifically target the ED-B domain of fibronectin. In previous studies L19 was used for imaging and targeted (radio-) 
immunotherapy, proving that L19 actually targets the tumour (29-31). Moreover, in phase I clinical studies in patients with metastatic melanoma or RCC, administration of the immunocytokine L19-IL2 solely or combined with chemotherapy (dacarbazine) was safe and showed clinical activity according to RECIST criteria or progression-free-survival $(32,33)$. Dacarbazine has however not the potential to induce an anti-tumour immune response, stimulate the exposure of DAMPs or activate ICD (34), which are all favourable characteristics induced by RT. Therefore, based on the known immunogenic effects of RT and the targeted immune stimulating potential of L19-IL2, we hypothesize that the combination of radiotherapy with L19-IL2 will cause an enhanced anti-tumour effect, which is dependent on the expression of ED-B.

\section{RESULTS}

Representative sections of the ED-B expression in the C51, LLC and 4T1 tumours and their respective fluorescent intensity, corrected for the intensity of the negative controls are shown in figure 1 . We observed a high, intermediate and low ED-B expression for the

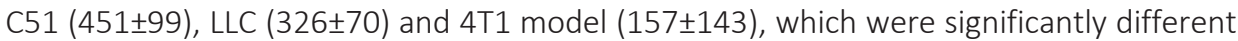
from each other (all $\mathrm{P}<0.01$ ). For none of the treatment combinations, toxicity was observed based on body weight measurements and animal well fare monitoring.

A
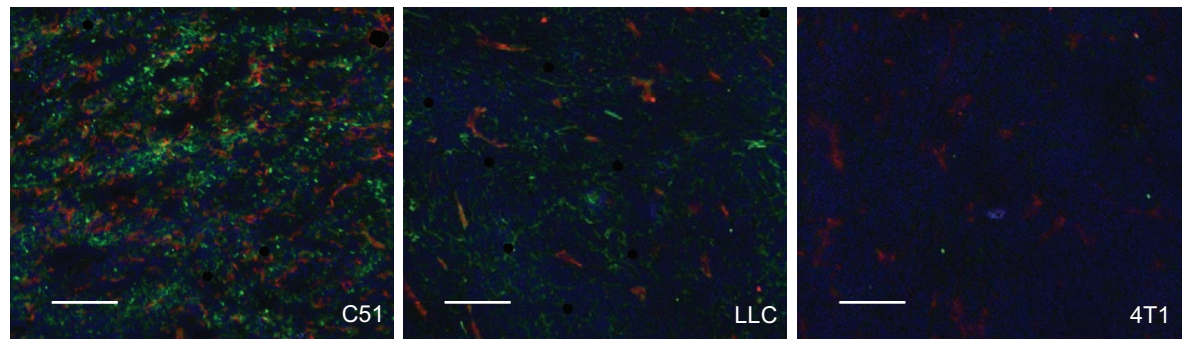

B

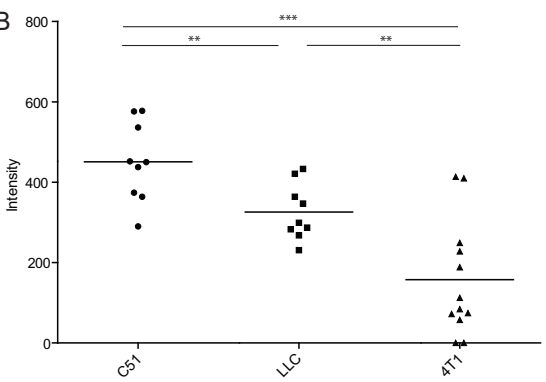

Figure 1 High, intermediate and low ED-B expressing tumour models. A: ED-B expression for C51 colon carcinoma, Lewis Lung Carcinoma (LLC) and 4T1 mammary carcinoma. ED-B expression is detected by L19(sip) biotinilated (green), vessels by anti-CD31 (red) and cell nuclei (DAPI staining) in blue. Scale bar $100 \mu \mathrm{m}$ B: Quantification of the ED-B expression (intensity) is corrected for negative control (KSF(sip) biotinilated). ${ }^{* *} \mathrm{P}<0.01,{ }^{* * *} \mathrm{P}<0.001$. 
Combination therapy results in complete remission of $75 \%$ in the $C 51$ model.

We evaluated the time to reach 4 times start volume (T4xSV) for all treatment groups in the C51 model with high ED-B expression. L19, IL2 or L19-IL2 monotherapy increased the T4xSV to $6.1 \pm 0.9(P<0.01), 6.3 \pm 1.2(P<0.01)$ and $6.0 \pm 1.6$ days $(P<0.05)$, respectively, as compared to the vehicle ( $4.8 \pm 0.8$ days) treated $\mathrm{C} 51$ tumour-bearing animals, while no significant differences between these three treatment groups were observed. Singledose radiotherapy (10Gy) significantly enhanced tumour growth delay when preceding vehicle $(P<0.001)$, L19 $(P<0.001)$ or IL2 $(P<0.001)$ treatment. Upon combination with L19-IL2 therapy, a highly significant $(P<0.0001)$ synergistic anti-tumour effect was observed with 9/12 cures (Figure 2A). Reduction of the single-dose radiations to 5 or 2 Gy showed a dose-dependent treatment effect. For tumours treated with the combination of ionizing radiation and L19-IL2, a cure rate of $6 / 12$ and $1 / 12$ was observed for irradiation with 5 Gy $(P<0.001)$ and 2 Gy $(P=0.002)$, respectively, as compared to the combination with vehicle treatment (Figure $2 \mathrm{~B}$ ).

FACS analysis was performed to evaluate the underlying immunological parameters. The percentage of baseline cytotoxic T cells in the tumour was $22.2 \pm 9.2 \%$ of $C D 45^{+}$cells in vehicle treated animals. Radiotherapy slightly enhanced the cytotoxic $T$ cell subpopulation (28.1 $\pm 5.7 \%)$, however, without being significant $(P=0.24)$. The percentage of cytotoxic $T$ cells during combination treatment was significantly higher than in vehicle $(38.6 \pm 10.8 \%, P<0.01)$ or L19-IL2 only $(22.0 \pm 8.8 \%, P=0.01)$ treated animals. There was no significant difference in the $\mathrm{CD}_{4} 5^{+}$population in the tumour between different treatment groups. In addition, no significant differences were observed in $\mathrm{NKp}_{4} 6^{+} \mathrm{NK}$ cells, $\mathrm{CD}^{+} \mathrm{T}$ cells or $\mathrm{CD}_{1} 9^{+} \mathrm{B}$-cells between the treatment groups (Figure $2 \mathrm{C}$ and Supplementary Table 1 ). Flow cytometry of the lymph node and spleen tissue showed no significant difference for any of the analyzed immune subpopulations (CD8 ${ }^{+}, \mathrm{CD}^{+}, \mathrm{CD}_{1} 9^{+}$and NK; Supplementary Table 1$)$. 
A

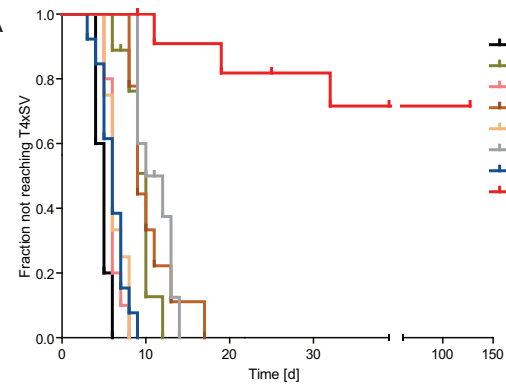

Cure (9/12)

B
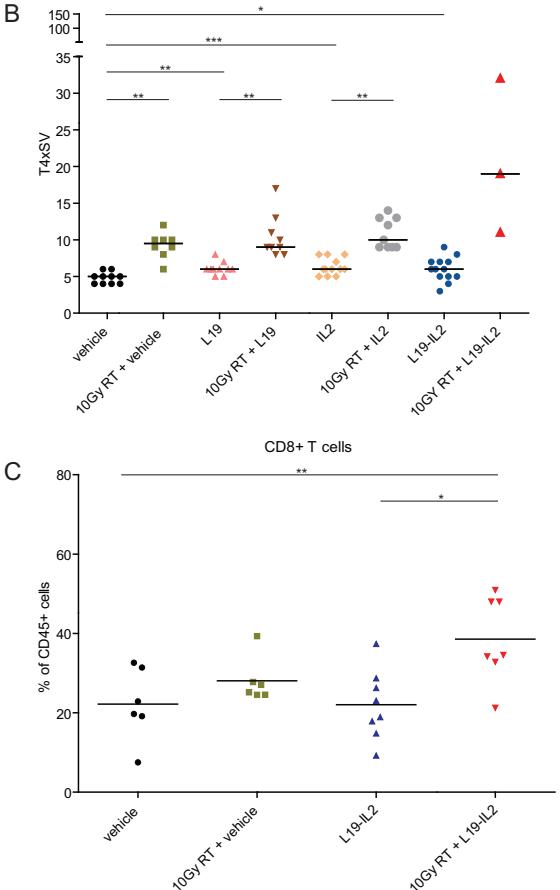

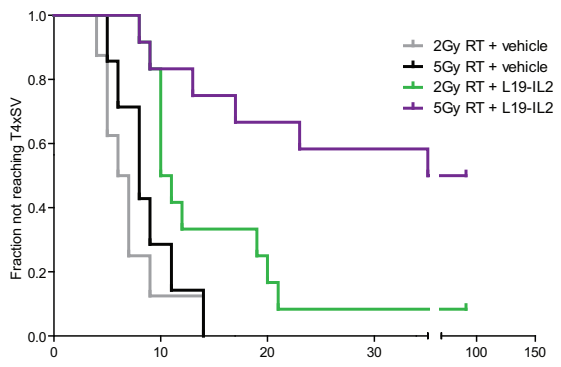

Cure (x/12) 6

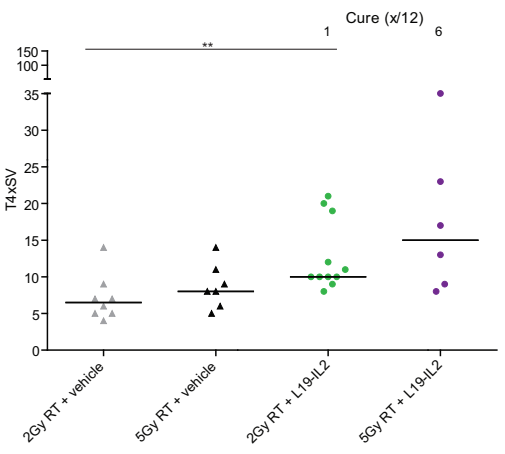

NKp46+ cells

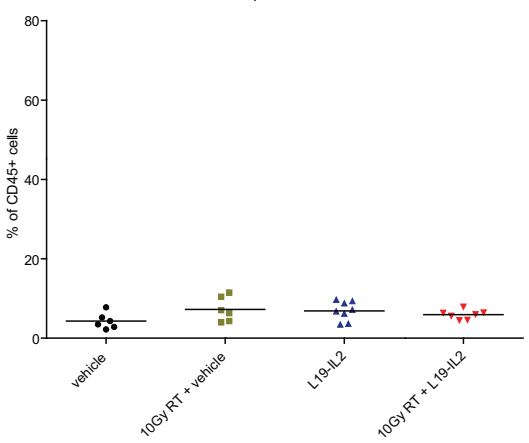

Figure 2: Combination therapy results in complete remission of 75\% in the C51 model. C51 colon carcinoma model. A: Fraction of tumours not reaching 4 times start-volume (T4xSV). B: Time to reach 4 times start volume for the different treatment groups. C: Results of flow cytometry analysis, shown is the percentage of $\mathrm{CD}^{+}$and $\mathrm{NKp} 46^{+}$cells of all $\mathrm{CD} 45^{+}$cells present in the tumour. Data represent the mean of $\mathrm{n}=6-12$ tumours. ${ }^{*} p<0.05, * * p<0.01, * * * p<0.001$. 
Combination therapy results in increased growth delay in LLC model.

Next, we investigated the possible therapeutic effect of combined RT with L19-IL2 in the LLC model with intermediate ED-B expression. There was no significant difference in tumour growth delay for L19 (4.3 \pm 1.2 days) or IL2 (5.1 \pm 1.1 days) compared to vehicle (4.4 \pm 1.1 days) treated animals. L19-IL2 monotherapy resulted in a significant tumour growth delay $(P=0.02)$, increasing T4XSV to $6.0 \pm 1.4$ days. Single-dose radiotherapy (10 Gy) only showed an increased growth delay (6.9 \pm 2.0 days; $P=0.02)$, however, the combination of RT with L19-IL2 resulted in the largest growth delay (10.5 \pm 2.6$)$. This was significantly longer than after RT or L19-IL2 only $(P<0.01$ and $P<0.001$, respectively; Figure 3A, 3B). There was no significant interaction between RT and L19-IL2 (2-way ANOVA; $P=0.15)$, the effect of the combination therapy in LLC was additive.

The observed baseline percentage of cytotoxic $\mathrm{CD}^{+} \mathrm{T}$ cells in this LLC model was significantly lower than in the $\mathrm{C} 51$ model $(\mathrm{P}=0.002)$. The number of cytotoxic $\mathrm{T}$ cells as a percentage of $\mathrm{CD} 5^{+}$cells increased significantly upon L19-IL2 treatment: from $2.7 \pm 1.0 \%$ (vehicle) to $7.4 \pm 4.1 \%$ (L19-IL2, P=0.04), and from $1.9 \pm 0.8 \%$ (RT) to $4.4 \pm 2.6 \%$ (RT+L19-IL2, P=0.04; figure 3C). Radiation caused a significant decrease in the percentage of $\mathrm{CD}_{1} 9^{+}$and $\mathrm{CD}^{+}$cells in the tumour compared to vehicle treatment (Supplementary Table 1). No differences were observed in the percentage of $\mathrm{NKp} 6^{+}$ cells in the tumour (Figure 3A, 3B). Analysis of the lymph nodes and spleen tissue showed no significant differences (Supplementary Table 1).

In the low/negative ED-B expressing $4 T 1$ model, the addition of L19-IL2 to radiotherapy has no effect.

Next, we investigated if L19-IL2 had any off-target effects using the low ED-B expressing 4T1 model. For the 4T1 model, no statistically significant differences were observed between vehicle, IL2 and L19-IL2 treated animals, with an average T4XSV of 7.9 \pm 2.8 , $8.7 \pm 1.6$ and $9.2 \pm 2.4$ days, respectively. Single-dose radiotherapy (10 Gy) increased growth delay significantly for all treatment groups: $R T+$ vehicle (13.3 \pm 3.7 days, $P=0.01)$,

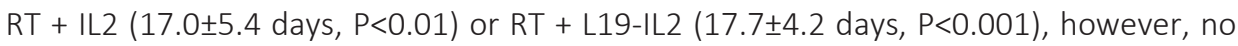
statistically significant differences $(P=0.47$ and $P=0.59)$ were observed between these irradiated groups (Figure $3 \mathrm{~A}, 3 \mathrm{~B}$ ). There is no significant interaction between RT and L19-IL2 (2-way ANOVA; $p=0.20$ ).

Radiotherapy caused a significant increase in the presence of $\mathrm{CD}^{+} \mathrm{T}$ cells in the 4T1 tumour. The percentage of $\mathrm{CD}^{+} \mathrm{T}$ cells increased from $6.9 \pm 1.8$ (vehicle) to $18.0 \pm 12.6$ (RT + vehicle, $P=0.04$ ) and from $6.4 \pm 2.9$ (L19-IL2) to 14.2 \pm 6.4 (RT + L19-IL2, $P<0.01)$. Albeit, no significant differences $(P=1.0)$ were observed for L19-IL2 treated animals compared to vehicle. No significant differences were observed for the percentage of NK cells in the tumour for any of the treatment groups (Figure $3 \mathrm{C}$ ). The percentage of 
CD19+ cells were significantly higher for treatment with L19-IL2 alone (4.0 \pm 1.6$)$ compared to vehicle (2.5 $\pm 0.6, \mathrm{P}=0.03), \mathrm{RT}+$ vehicle $(2.0 \pm 0.7, \mathrm{P}=0.02)$ and $\mathrm{RT}+\mathrm{L} 19-\mathrm{IL} 2$ (1.6 $\pm 1.2, P=0.04$; Supplementary Table 1). Analysis of the spleen and lymph nodes showed no significant difference for any of the analyzed immune cells.
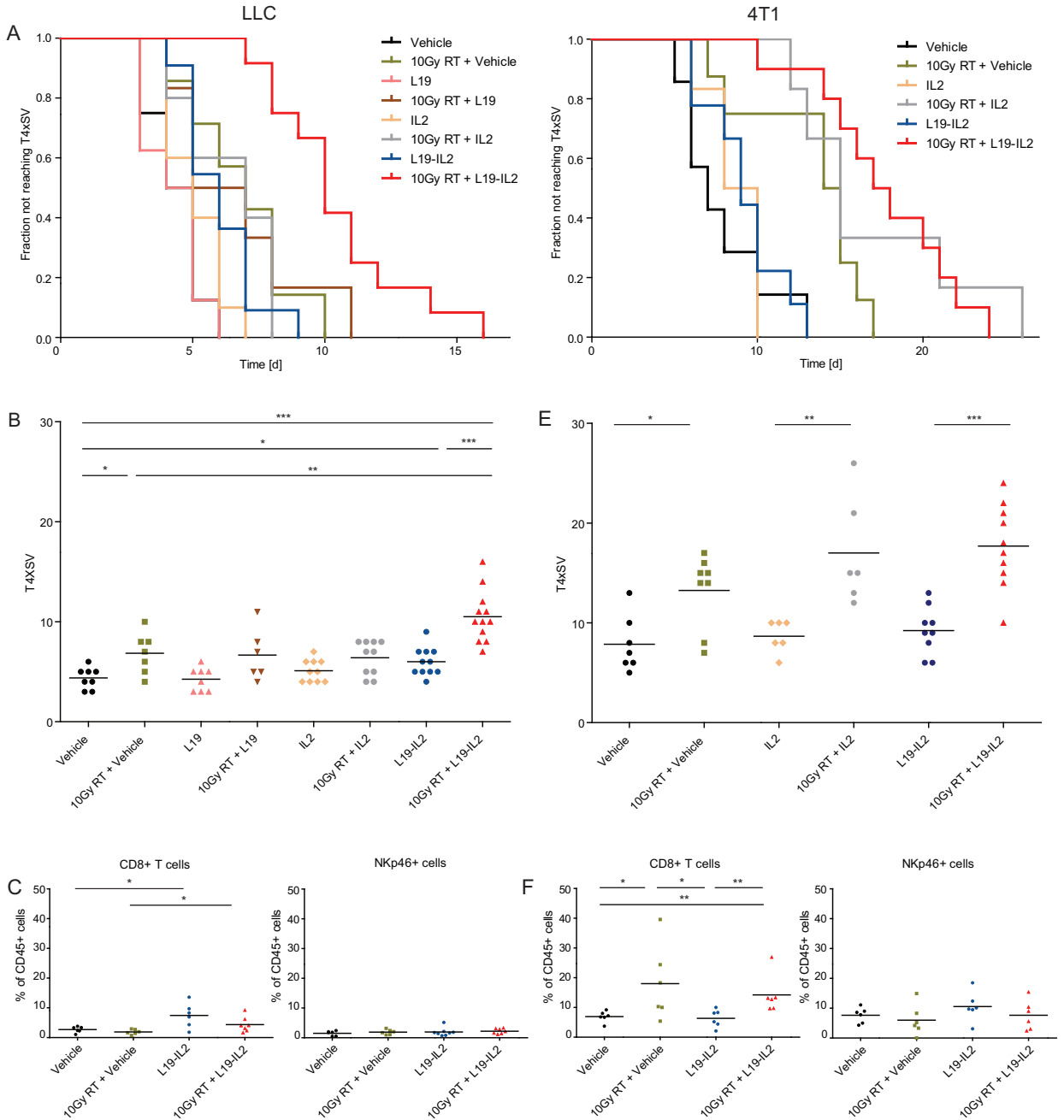

Figure 3: Combination therapy: Additive effect in LLC model (intermediate ED-B) and no effect in the 4T1 model (low ED-B). Lewis Lung Carcinoma (LLC), and 4T1 mammary carcinoma model. Survival curves LLC model (A) and 4T1 model (D), showing the fraction of tumours not reaching 4 times start-volume (T4XSV). Scatter-plots LLC model (B) and 4T1 model (E), showing the time to reach 4 times start volume (T4xSV). Flow cytometric analyses of tumour leukocyte content in the LLC (C) and the 4T1 (F) model, showing the percentage of $\mathrm{CD}^{+}$and $\mathrm{NKp} 46^{+}$cells of al $\mathrm{CD} 45^{+}$cells in the tumour. ${ }^{*} p<0.05,{ }^{* *} p<0.01,{ }^{* * *} p<0.001$. 
Depletion of cytotoxic T cells prohibits complete remission.

Based on our observation that radiotherapy + L19-IL2 immunotherapy significantly increases the $\mathrm{CD}^{+} \mathrm{T}$ cell subpopulation, we assessed the causal relationship between the therapeutic effect and $\mathrm{CD8} 8^{+} \mathrm{T}$ cells by depleting the $\mathrm{CD} 8^{+} \mathrm{T}$ cells in the $\mathrm{C} 51$ tumour model. Tumour cell injection did not result in changed immune subpopulations (Supplementary figure 4B). Treatment with the $\mathrm{CD8}^{+} \mathrm{T}$ cell depleting (JTS169) antibody abolished $\mathrm{CD}^{+} \mathrm{T}$ cells in the blood 2 days after injection (0.06 $\pm 0.06 \%$; $>99 \%$ depletion). Cytotoxic $\mathrm{CD} 8^{+} \mathrm{T}$ cells were detectable again at day $3(1.6 \pm 0.7 \%)$ after depletion and levels increased over time to $5.7 \pm 3.0 \%$ at day 5 (Figure $4 \mathrm{~A}$ ). On the basis of these data we opted for 3-daily administration of $\mathrm{CDB}^{+}$depleting antibody in order to effect sustained ablation of $\mathrm{CD} 8^{+} \mathrm{T}$ cells (Figure $4 \mathrm{~B}$ ). After depletion ( 3 days p.i.), the $\mathrm{CD} 8^{+} \mathrm{T}$ cell population was significantly reduced in blood $(\mathrm{P}<0.0001)$, whereas, the control groups receiving either isotype IgG $(15.7 \pm 0.9 \%)$ or vehicle $(18.1 \pm 3.1 \%)$ showed similar numbers of $\mathrm{CD} 8^{+}$cells as baseline (Figure $4 \mathrm{C}$ ). $\mathrm{CD}^{+} \mathrm{T}$ cells were also depleted in the tumour $(2.2 \pm 2.6 \%$, vs $38.6 \pm 10.8 \%$ at baseline; Figure $4 \mathrm{D})$, spleen and lymph nodes (Supplementary Figure 4). Upon depletion of cytotoxic $T$ cells, the combination of radiotherapy with L19-IL2 lost its therapeutic effect (T4XSV=11.25 $\pm 3.0 \mathrm{~d}$ ) and was not superior $(P=0.31)$ to radiotherapy only $(T 4 \times S V=10.0 \pm 3.0 d)$. However, in agreement with previous results, the animals in the control groups (without $\mathrm{CD} 8^{+} \mathrm{T}$ cell depletion), still showed sustained anti-tumour effects (IgG: cure 5/8, vehicle: cure 3/8) after $10 \mathrm{~Gy}$ irradiation and L19-IL2 (Figure 4E). 
A

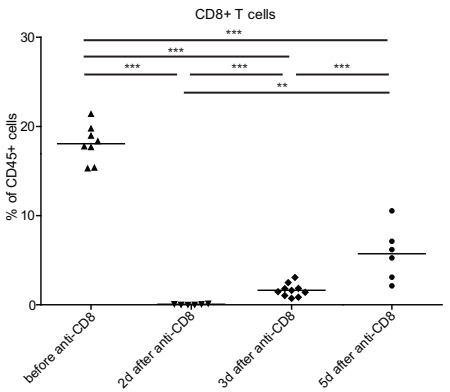

C

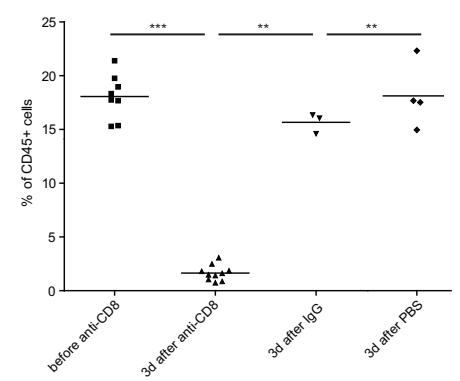

D

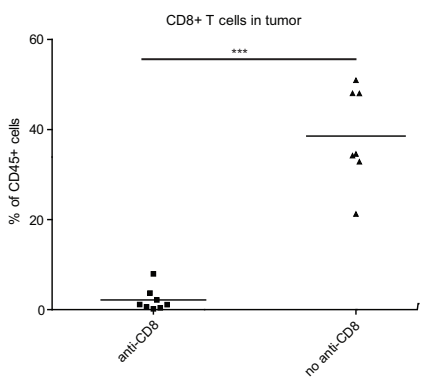

B
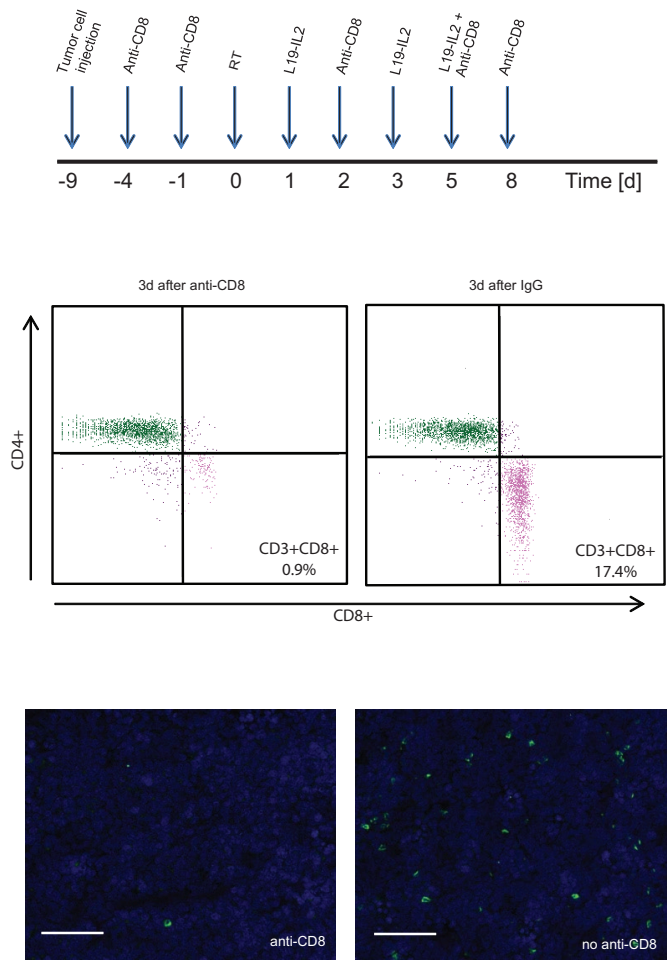

Cure rates:

$5 / 8 \quad 3 / 8$

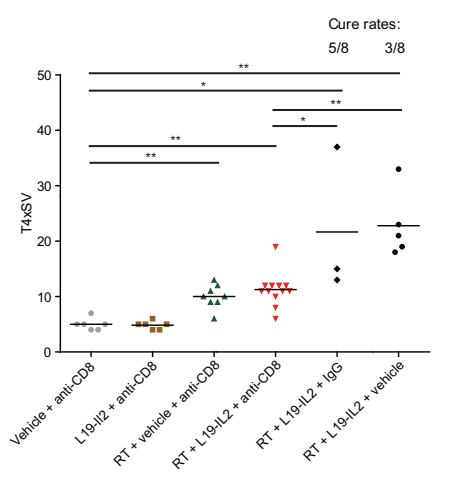

E

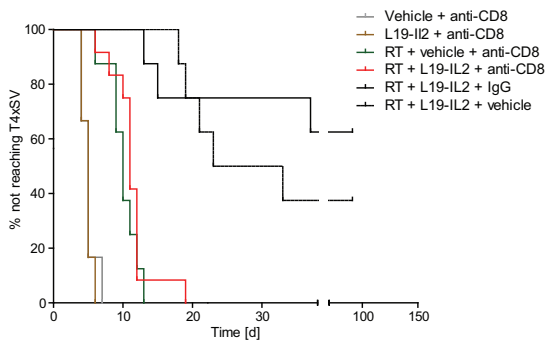

Figure 4: Depletion of cytotoxic T cells prohibits complete remission. CD8 depletion in the C51 model. A: \% of $\mathrm{CD}^{+}$cells of $\mathrm{CD} 5^{+}$cells 2,3 and 5 days after single i.p. anti-CD8 administration. B: Cartoon of treatment schedule. C: \% $C D 8^{+}$cells of $C D 45^{+}$cells 3 days after i.p. anti-CD8, IgG or PBS (vehicle) and an example of the flow cytometry results showing the percentage of $\mathrm{CD}^{+} \mathrm{CD}^{+}$cells present in the blood 3 days after anti-CD 8 or IgG administration. D: \% of $\mathrm{CD}^{+}$cells present in the tumour of CD8 depleted and non-depleted mice treated with RT and L19-IL2 analyzed by flow cytometry and an immunofluorescent CD8 staining (green), cell nuclei stained with DAPI (blue). E: Fraction of tumours not reacting $4 \mathrm{x}$ start volume (T4xSV) and time to reach T4xSV for the different treatment groups. ${ }^{*} p<0.05,{ }^{* *} p<0.01,{ }^{* * *} p<0.001$ 


\section{DISCUSSION}

Radiation-induced cell death is an immunogenic process which can be used to initiate tumour-specific immune responses (35). The selective delivery of IL2 to tumour vascular components is promising in cancer immunotherapy $(15,36,37)$ and may be used to enhance the therapeutic potential of RT. We hypothesized that the combination of RT with the targeted immunocytokine L19-IL2 may cause an enhanced anti-tumour effect dependent on the expression of ED-B. In this study, we assessed the therapeutic potential and underlying mechanisms of the combination therapy in three different tumour models with varying ED-B expression.

Based on growth delay experiments, the combination therapy showed a therapeutic gain compared with the single treatment arms with an additive effect for the LLC model and a long-lasting highly synergistic effect for the C51 model for which a cure rate of $75 \%$ was observed. As expected no effect was observed for the 4T1 model, which has a low ED-B expression. The results show that ED-B expression is essential for the efficacy of combined irradiation and L19-IL2 administration. The C51 model showed the highest ED-B expression and the most promising results for the combination therapy suggesting that high ED-B expression may assure a better L19-IL2 tumour targeting. Like our C51 model, ED-B is overexpressed in many solid tumours $(23-25,27,37)$, which makes this combination therapy (RT + L19-IL2) potentially interesting for the majority of cancer types.

The highly synergistic effect observed in the C51 model upon radiotherapy and tumourtargeted L19-IL2 treatment is in agreement with previous results described by Yasuda et al. (21). They observed a complete eradication of a colon carcinoma cell line (Colon26) in Balb/c mice after the combination of radiotherapy with intra-tumoural injections with IL2. For the models presented in this study, no additional benefit was observed for the use of the single treatment with L19-IL2 in comparison to IL2 treatment. This is in contradiction with the results from previous studies, showing that L19-IL2 provides a stronger anti-tumour effect compared with equimolar dosing of untargeted IL-2 in an F9 teratocarcinoma or a human pancreatic carcinoma xenograft model $(15,38)$. This might be explained by the use of different mouse strains, tumour models and treatment schedules. However, in combination with radiotherapy, we did find a stronger antitumour effect when using L19-IL2 compared to IL2. This shows that, in agreement with previous results, L19-IL2 has an increased anti-tumour effect.

Upon combination therapy an increased number of cytotoxic T cells was observed in the tumour of the LLC and C51 model. A comparison between the used models shows that already at baseline, the number of cytotoxic T cells is higher for the C51 model than for the LLC and 4T1 models. Results are in agreement with previous publications, where it was already shown that, dependent on tumour model, the efficacy of IL2 treatment can 
be based on T cells $(39,40)$, or a combination of NK and T cells $(15)$. In mice bearing C51 colon carcinoma, L19-IL2 as single treatment already showed an increased number of tumour-infiltrating cytotoxic T- and NK cells in immunohistochemical analysis (15), which was confirmed in the clinical setting where both cell types were up-regulated in the peripheral blood of patients as a result of L19-IL2 treatment (32). Johnson et al. (41) combined an alternative immunocytokine, KS-IL2, with radiofrequency ablation in a murine colon adenocarcinoma (CT26). The combination increased growth suppression, and a greater proportion of $\mathrm{CD}^{+}$and $\mathrm{CD}^{+}$cells was observed. Furthermore, the therapeutic effect of IL2 coupled to the human monocloncal antibodies F8 and F16 that recognize the ED-A and ED-B domains of fibronectin and the A1 domain of tenascin-C, respectively, was shown to be mediated by $\mathrm{CD}^{+}$and $\mathrm{NK}$ cells in an in vivo $\mathrm{AML}$ model (42). Moreover, the antibody-based targeted delivery of IL4 and IL12 to tumour neovasculature has also shown to eradicate tumours by both NK and $\mathrm{CD}^{+} \mathrm{T}$ cells (43). In our study, we irradiated the tumours prior to administration of the immunocytokine L19-IL2. It is known that radiotherapy can promote a DC-mediated cytotoxic Tlymphocyte (CTL) response, the so-called immunogenic cell death (44). This form of cell death may be further enhanced by the targeted delivery of IL2 to the irradiated tumours. Our combination therapy may therefore favour the CTL response, because NK cells are able to detect and destroy malignant and virally infected cells directly (14). Indeed we have shown that depletion of the cytotoxic T cells in the C51 model inhibits the anti-tumour effect after combination therapy, providing evidence that the complete remission observed in the majority of C51 tumours, is attributed to the high number of cytotoxic $T$ cells present in the tumour after combination therapy.

Evidence suggests that local radiation always elicits activation of the immune system, even though the proportion of tumour cells undergoing immunogenic cell death will vary $(4,6)$. Demaria et al. (6) showed that a single low dose of RT (2Gy) in combination with Flt3-Ligand (enhancing the number of available dendritic cells) could already trigger anti-tumour $\mathrm{T}$ cell responses. To test this hypothesis in our study, the radiotherapy dose was reduced from 10Gy to 5Gy or 2Gy for the C51 model. The decrease in dose of irradiation resulted in a reduced number of tumour eradications, showing that in this model and experimental set-up the radiotherapy dose is an important parameter to generate cure. We therefore suggest that a minimal radiotherapy dose is necessary to provide sufficient immunogenic cell death to trigger the anti-tumour immune response. In our experiments, we only tested one single RT dose in combination with L19-IL2, showing excellent results. Therefore we expect that the use of a few high doses of radiotherapy (SBRT) is sufficient to release DAMPs and initiate the anti-tumour immune response, while limiting the damage to essential immunological $\left(\mathrm{CD}^{+}\right)$cells. In a previous clinical trial, Seung et al (45) combined SBRT with systemic IL2 in patients with metastatic melanoma or RCC, this provided already a higher response rate compared to historical data. Based on our results the use of L19- 
IL2, instead of systemic IL2, will increase the potential and decrease toxicity. Therefore the clinical set-up combining SBRT with L19-IL2 seems very promising and will be investigated in a clinical trial (NCT02086721).

Since ED-B has an identical amino sequence in mice and humans, the human single chain Fv monoclonal antibody fragment L19 combined with IL2 can be directly used in clinical setting. In Phase I trials, L19-IL2 was already safely administered in melanoma and renal cell carcinoma, even in combination with decarbazine, which is not an ICD inducer like RT (32-34). Based on our current results that ED-B expression is essential to obtain a therapeutic benefit, L19-SIP imaging should be included in a clinical trial set-up, to evaluate the possibility to select patients for L19-IL2 treatment. The ultimate aim is however to increase progression fee survival by the irradiation of accessible, larger solid tumours/metastasis, initiating an anti-tumour immune response which will attack the solid lesions and its micro-metastasis.

In conclusion, the combination therapy of RT with L19-IL2 can enhance the immune response against diverse solid tumours, providing an additive or synergistic anti-tumour effect in the presence of ED-B. These findings can directly be translated to a Phase I clinical study in patients with an oligometastatic solid tumour, since the use of L19-IL2 is proven to be safe in patients. This promising new opportunity for cancer treatment is subject of clinical investigation.

\section{MATERIALS AND METHODS}

\section{Tumour cell lines.}

Exponentially growing C51 colon carcinoma (kindly provided by Philogen S.p.A., Siena, Italy), Lewis lung carcinoma (LLC; kindly provided by G. Molema, UMCG, The Netherlands), and 4T1 mammary carcinoma (ATCC CRL-2539) cell lines were cultured in Dulbecco's Modified Eagle Medium (DMEM; Lonza) supplemented with $10 \%$ fetal calf serum (FCS) in a humidified $5 \% \mathrm{CO}_{2}$ chamber at $37^{\circ} \mathrm{C}$.

\section{Chemicals/reagents/antibodies.}

The L19-IL2 immunocytokine and L19 (Philogen S.p.A.) were diluted with sterile phosphate buffered saline (PBS, Lonza) to concentrations of respectively $200 \mu \mathrm{g} / \mathrm{ml}$ and $133 \mu \mathrm{g} / \mathrm{ml}$. Interleukin-2 (Proleukin, Novartis, Basel, Switserland) was dissolved as described by the manufacturers' guidelines and diluted with PBS to a concentration of $67 \mu \mathrm{g} / \mathrm{ml}$. 
For the depletion experiments, the anti-CD8 antibody (Clone JTS169) and the isotype control (anti-Phyt IgG AFRC MAC 51), kindly provided by Bioceros Bv, Utrecht, The Netherlands, were diluted with PBS to a concentration of $2 \mathrm{mg} / \mathrm{ml}$.

Flow-cytometric analysis was performed on cells exposed to RBC lysis buffer (eBioscience, San Diego, CA), FC-block CD16/CD32 (BD Biosciences, San Jose, CA) and a combination of the antibodies CD3-FITC, CD4-APC-H7, CD8-PE-CY7, CD19-PE, CD45V500, CD45-Percp, CD45-PE, CD45-FITC, CD45-APC, CD45-PE-CY7, CD3e-eFLU0450, CD4-FITC, CD8a-V500 (BD Biosciences) and NKp46-APC (Miltenyi Biotec B.V., Leiden, The Netherlands).

\section{In vivo experiments.}

All experiments were performed in accordance with local institutional guidelines for animal welfare and were approved by the Animal Ethical Committee of the University of Maastricht. To induce tumours, approximately 8 weeks old immune competent mice were subcutaneously injected with syngeneic C51 (Balb/c; 1.5×10 $)$, LLC (C57bl/6; $0.5 \times 10^{6}$ ) or $4 \mathrm{~T} 1$ (Balb/c; $1 \times 10^{6}$ ) tumour cells, resuspended in Basement Membrane Matrix (MatrigelTM, BD Biosciences). Upon an average tumour volume of $200 \mathrm{~mm}^{3}$, tumours were irradiated with a single dose (10 Gy for all models, additional groups with

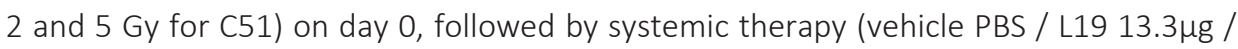

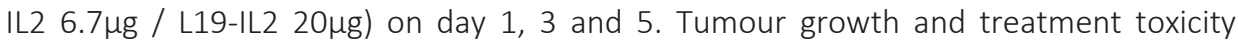
(based on body-weight) were monitored until reaching 4 times the volume at irradiation time (T4xSV). Flow cytometry was performed on tumours, spleen and lymph nodes excised at day 4 of the treatment schedule. Detailed treatment schedules are shown in supplementary figure 1.

To evaluate the causal relationship between the presence of cytotoxic $T$ cells and tumour growth delay, an experimental set-up was designed to deplete cytotoxic-T cells in the Balb/c mice bearing C51 tumours. Similar to previous experiments, local irradiation was performed on day 0 (10 Gy) and systemic therapy (vehicle or L19-IL2) was administrated (day $1,2,5$ ). In addition, $\mathrm{CD}^{+}$cells were depleted by intraperitoneal injection of $0.2 \mathrm{mg}(100 \mu \mathrm{L})$ anti-CD8 antibody or the negative control anti-Phyt IgG. The timing for the anti-CD8 injections was determined by blood withdrawal, via puncture of the saphenous vein (i) before, (ii) after tumour cell injection and (iii) 2,3 or 5 days after injection with the blocking antibodies. The percentage $\mathrm{CD}^{+}$cells in the blood was determined as described below. At the end of the experiment the tumours were harvested for immunohistochemical analysis for CD8 positivity. 
Flow cytometry.

The number of immune cell subpopulations present in tumour, spleen and lymph nodes during treatment was analyzed using FACSCanto II flow cytometry (FACS, BD Biosciences). Single cell suspensions of the tissues were obtained using the gentleMACS dissociator and the tumour dissociation kit (Miltenyi Biotec B.V.) according to manufacturer's guidelines. Of the acquired single cell suspension, $1.0 \times 10^{6}$ cells were suspended in FACS buffer (PBS $+1 \%$ FCS) for analysis. Cells were incubated with FCblock to avoid non-specific binding and staining was performed using the antibodies CD3-FITC, CD4-APC-H7, CD8-PE-CY7, CD19-PE, NKp46-APC and CD45-V500. The total $\mathrm{CD} 45^{+}$immune cells were selected from the viable population of cells (filtered for debris and doublets) for further sub-classification according to the strategy described in supplementary figure 2 .

To determine the efficacy of anti-CD8 blocking antibody on the presence of specific immune subpopulations, collected blood was incubated with RBC lysis buffer and FCblock. Next cells were incubated with CD45-Percp, CD3e-eFLU0450, CD4-FITC, CD8aV500, NKp46-APC and CD19-PE, and FACS and data analysis was performed (Supplementary Figure 3).

\section{Immunofluorescence.}

To investigate baseline ED-B expression, $7 \mathrm{~mm}$ cryostat sections of C51, LLC and 4T1 tumours were fixed in acetone $\left(4^{\circ} \mathrm{C}\right)$ and stained according to previous published methods (46). In brief, sections were incubated with the purified antibodies L19-SIP or KSF-SIP (2ug/ml; Philochem, Otelfingen, Switzerland), with rabbit anti-human-IgE (Dako, Glostrup, Denmark) and subsequently detected using goat anti-rabbit IgG Alexa Fluor 488 (Life Technologies, Bleiswijk, The Netherlands). Blood vessels and cell nuclei were detected with rat anti-mouse CD31 (BD Biosciences) followed by donkey anti-rat Alexa 594 (Life Technologies) and DAPI (Life Technologies), respectively.

To quantify the ED-B expression, 3-12 photomicrographs (805.5 $\mu \times 805.5 \mu)$, depending on tumour size, from viable tumour regions in the largest tumour cross-section were acquired using an Olympus BX51WI (Center Valley, PA) fluorescence microscope equipped with a Hamamatsu EM-CCD C9100 digital camera, a motorized stage (Ludl Mac 2000) and a 10x objective. Micromanager 1.4 software was used for automated image acquisition (47). All image recordings were performed with the same settings and analyzed by an investigator blinded to the subject coding. Images were processed using ImageJ software v.1.49b ( $\mathrm{NIH}$, Bethesda, MD). The mean fluorescent intensity after correction for cutting and staining artefacts per image was averaged over all images per section to obtain ED-B intensity per tumour. 
For the detection of $\mathrm{CD}^{+} \mathrm{T}$ cells residing in tumours, sections were first incubated with anti-CD8 (clone 53.62.7, Department of Pathology, MUMC, Maastricht, The Netherlands) and visualized with goat anti-rabbit IgG Alexa Fluor 488 (Life Technologies). DAPI was used as nuclear counterstain.

\section{Statistics.}

Statistical analyses were performed using GraphPad Prism Software (v5.03, San Diego, CA). For all parameters mean \pm standard deviation (SD) are reported. The nonparametric Mann-Whitney test was used to determine the statistical differences between the different treatment groups. The log-rank (Mantel-Cox) test was used to compare the survival curves. We used a 2-way ANOVA to test the interaction (synergism) between radiotherapy and L19-IL2. A p-value smaller than 0.05 was considered statistically significant.

\section{ACKNOWLEDGEMENTS}

Authors acknowledge financial support from Philogen S.p.A. (Sovicille, Italy), EU 6th and 7th framework program (METOXIA, ARTFORCE). The micrographs were taken with a confocal spinning disk microscope financed by The Netherlands Organisation for Scientific Research (NWO), grant number 911-06-003. 


\section{REFERENCES}

1. Krysko, D.V., Garg, A.D., Kaczmarek, A., Krysko, O., Agostinis, P., and Vandenabeele, P. 2012. Immunogenic cell death and DAMPs in cancer therapy. Nature reviews. Cancer 12:860-875.

2. Kaur, P., and Asea, A. 2012. Radiation-induced effects and the immune system in cancer. Frontiers in oncology 2:191.

3. Demaria, S., Bhardwaj, N., McBride, W.H., and Formenti, S.C. 2005. Combining radiotherapy and immunotherapy: a revived partnership. International journal of radiation oncology, biology, physics 63:655-666.

4. Demaria, S., and Formenti, S.C. 2012. Role of T lymphocytes in tumor response to radiotherapy. Frontiers in oncology 2:95.

5. Green, D.R., Ferguson, T., Zitvogel, L., and Kroemer, G. 2009. Immunogenic and tolerogenic cell death. Nature reviews. Immunology 9:353-363.

6. Demaria, S., Ng, B., Devitt, M.L., Babb, J.S., Kawashima, N., Liebes, L., and Formenti, S.C. 2004. Ionizing radiation inhibition of distant untreated tumors (abscopal effect) is immune mediated. International journal of radiation oncology, biology, physics 58:862-870.

7. Wersall, P.J., Blomgren, H., Pisa, P., Lax, I., Kalkner, K.M., and Svedman, C. 2006. Regression of nonirradiated metastases after extracranial stereotactic radiotherapy in metastatic renal cell carcinoma. Acta oncologica 45:493-497.

8. Okuma, K., Yamashita, H., Niibe, Y., Hayakawa, K., and Nakagawa, K. 2011. Abscopal effect of radiation on lung metastases of hepatocellular carcinoma: a case report. Journal of medical case reports 5:111.

9. Cotter, S.E., Dunn, G.P., Collins, K.M., Sahni, D., Zukotynski, K.A., Hansen, J.L., O'Farrell, D.A., Ng, A.K., Devlin, P.M., and Wang, L.C. 2011. Abscopal effect in a patient with metastatic Merkel cell carcinoma following radiation therapy: potential role of induced antitumor immunity. Archives of dermatology 147:870-872.

10. Kachikwu, E.L., Iwamoto, K.S., Liao, Y.P., DeMarco, J.J., Agazaryan, N., Economou, J.S., McBride, W.H., and Schaue, D. 2011. Radiation enhances regulatory T cell representation. International journal of radiation oncology, biology, physics 81:1128-1135.

11. Postow, M.A., Harding, J., and Wolchok, J.D. 2012. Targeting immune checkpoints: releasing the restraints on anti-tumor immunity for patients with melanoma. Cancer journal 18:153-159.

12. Stamell, E.F., Wolchok, J.D., Gnjatic, S., Lee, N.Y., and Brownell, I. 2013. The abscopal effect associated with a systemic anti-melanoma immune response. International journal of radiation oncology, biology, physics 85:293-295.

13. Topham, N.J., and Hewitt, E.W. 2009. Natural killer cell cytotoxicity: how do they pull the trigger? Immunology 128:7-15.

14. Janeway, C.A., Travers, P., Walport, M., and Shlomchik, M.J. 2001. Immunobiology: The Immune System in Health and Disease: Garland Science New York.

15. Carnemolla, B., Borsi, L., Balza, E., Castellani, P., Meazza, R., Berndt, A., Ferrini, S., Kosmehl, H., Neri, D., and Zardi, L. 2002. Enhancement of the antitumor properties of interleukin-2 by its targeted delivery to the tumor blood vessel extracellular matrix. Blood 99:1659-1665.

16. Wang, K.S., Ritz, J., and Frank, D.A. 1999. IL-2 induces STAT4 activation in primary NK cells and NK cell lines, but not in T cells. Journal of immunology 162:299-304.

17. Payne, R., Glenn, L., Hoen, H., Richards, B., Smith, J.W., 2nd, Lufkin, R., Crocenzi, T.S., Urba, W.J., and Curti, B.D. 2014. Durable responses and reversible toxicity of high-dose interleukin-2 treatment of melanoma and renal cancer in a Community Hospital Biotherapy Program. Journal for immunotherapy of cancer 2:13.

18. Alwan, L.M., Grossmann, K., Sageser, D., Van Atta, J., Agarwal, N., and Gilreath, J.A. 2014. Comparison of acute toxicity and mortality after two different dosing regimens of high-dose interleukin-2 for patients with metastatic melanoma. Targeted oncology 9:63-71. 
19. Den Otter, W., Jacobs, J.J., Battermann, J.J., Hordijk, G.J., Krastev, Z., Moiseeva, E.V., Stewart, R.J., Ziekman, P.G., and Koten, J.W. 2008. Local therapy of cancer with free IL-2. Cancer immunology, immunotherapy: Cll 57:931-950.

20. Jacobs, J.J., Sparendam, D., and Den Otter, W. 2005. Local interleukin 2 therapy is most effective against cancer when injected intratumourally. Cancer immunology, immunotherapy: CII 54:647-654.

21. Yasuda, K., Nirei, T., Tsuno, N.H., Nagawa, H., and Kitayama, J. 2011. Intratumoral injection of interleukin2 augments the local and abscopal effects of radiotherapy in murine rectal cancer. Cancer science 102:1257-1263.

22. Kontermann, R.E. 2012. Antibody-cytokine fusion proteins. Archives of biochemistry and biophysics 526:194-205.

23. Kaczmarek, J., Castellani, P., Nicolo, G., Spina, B., Allemanni, G., and Zardi, L. 1994. Distribution of oncofetal fibronectin isoforms in normal, hyperplastic and neoplastic human breast tissues. International journal of cancer. Journal international du cancer 59:11-16.

24. D'Ovidio, M.C., Mastracchio, A., Marzullo, A., Ciabatta, M., Pini, B., Uccini, S., Zardi, L., and Ruco, L.P. 1998. Intratumoral microvessel density and expression of ED-A/ED-B sequences of fibronectin in breast carcinoma. European journal of cancer 34:1081-1085.

25. Pujuguet, P., Hammann, A., Moutet, M., Samuel, J.L., Martin, F., and Martin, M. 1996. Expression of fibronectin ED-A+ and ED-B+ isoforms by human and experimental colorectal cancer. Contribution of cancer cells and tumor-associated myofibroblasts. The American journal of pathology 148:579-592.

26. Santimaria, M., Moscatelli, G., Viale, G.L., Giovannoni, L., Neri, G., Viti, F., Leprini, A., Borsi, L., Castellani, P., Zardi, L., et al. 2003. Immunoscintigraphic detection of the ED-B domain of fibronectin, a marker of angiogenesis, in patients with cancer. Clinical cancer research : an official journal of the American Association for Cancer Research 9:571-579.

27. El-Emir, E., Dearling, J.L., Huhalov, A., Robson, M.P., Boxer, G., Neri, D., van Dongen, G.A., Trachsel, E., Begent, R.H., and Pedley, R.B. 2007. Characterisation and radioimmunotherapy of L19-SIP, an antiangiogenic antibody against the extra domain B of fibronectin, in colorectal tumour models. British journal of cancer 96:1862-1870.

28. Galler, K., Junker, K., Franz, M., Hentschel, J., Richter, P., Gajda, M., Gohlert, A., von Eggeling, F., Heller, R., Giavazzi, R., et al. 2012. Differential vascular expression and regulation of oncofetal tenascin-C and fibronectin variants in renal cell carcinoma (RCC): implications for an individualized angiogenesis-related targeted drug delivery. Histochemistry and cell biology 137:195-204.

29. Rossin, R., Berndorff, D., Friebe, M., Dinkelborg, L.M., and Welch, M.J. 2007. Small-animal PET of tumor angiogenesis using a (76)Br-labeled human recombinant antibody fragment to the ED-B domain of fibronectin. Journal of nuclear medicine : official publication, Society of Nuclear Medicine 48:1172-1179.

30. Tijink, B.M., Perk, L.R., Budde, M., Stigter-van Walsum, M., Visser, G.W., Kloet, R.W., Dinkelborg, L.M., Leemans, C.R., Neri, D., and van Dongen, G.A. 2009. (124)I-L19-SIP for immuno-PET imaging of tumour vasculature and guidance of (131)I-L19-SIP radioimmunotherapy. European journal of nuclear medicine and molecular imaging 36:1235-1244.

31. Balza, E., Carnemolla, B., Mortara, L., Castellani, P., Soncini, D., Accolla, R.S., and Borsi, L. 2010. Therapyinduced antitumor vaccination in neuroblastomas by the combined targeting of IL-2 and TNFalpha. International journal of cancer. Journal international du cancer 127:101-110.

32. Johannsen, M., Spitaleri, G., Curigliano, G., Roigas, J., Weikert, S., Kempkensteffen, C., Roemer, A., Kloeters, C., Rogalla, P., Pecher, G., et al. 2010. The tumour-targeting human L19-IL2 immunocytokine: preclinical safety studies, phase I clinical trial in patients with solid tumours and expansion into patients with advanced renal cell carcinoma. European journal of cancer 46:2926-2935.

33. Eigentler, T.K., Weide, B., de Braud, F., Spitaleri, G., Romanini, A., Pflugfelder, A., Gonzalez-Iglesias, R., Tasciotti, A., Giovannoni, L., Schwager, K., et al. 2011. A dose-escalation and signal-generating study of the immunocytokine L19-IL2 in combination with dacarbazine for the therapy of patients with metastatic melanoma. Clinical cancer research : an official journal of the American Association for Cancer Research 17:7732-7742. 
34. Hervieu, A., Mignot, G., and Ghiringhelli, F. 2013. Dacarbazine mediate antimelanoma effects via NK cells. Oncoimmunology 2:e23714.

35. Kamrava, M., Bernstein, M.B., Camphausen, K., and Hodge, J.W. 2009. Combining radiation, immunotherapy, and antiangiogenesis agents in the management of cancer: the Three Musketeers or just another quixotic combination? Molecular bioSystems 5:1262-1270.

36. Schliemann, C., Palumbo, A., Zuberbuhler, K., Villa, A., Kaspar, M., Trachsel, E., Klapper, W., Menssen, H.D., and Neri, D. 2009. Complete eradication of human B-cell lymphoma xenografts using rituximab in combination with the immunocytokine L19-IL2. Blood 113:2275-2283.

37. Menrad, A., and Menssen, H.D. 2005. ED-B fibronectin as a target for antibody-based cancer treatments. Expert opinion on therapeutic targets 9:491-500.

38. Wagner, K., Schulz, P., Scholz, A., Wiedenmann, B., and Menrad, A. 2008. The targeted immunocytokine L19-IL2 efficiently inhibits the growth of orthotopic pancreatic cancer. Clinical cancer research : an official journal of the American Association for Cancer Research 14:4951-4960.

39. Becker, J.C., Pancook, J.D., Gillies, S.D., Furukawa, K., and Reisfeld, R.A. 1996. T cell-mediated eradication of murine metastatic melanoma induced by targeted interleukin 2 therapy. The Journal of experimental medicine 183:2361-2366.

40. Xiang, R., Lode, H.N., Dolman, C.S., Dreier, T., Varki, N.M., Qian, X., Lo, K.M., Lan, Y., Super, M., Gillies, S.D., et al. 1997. Elimination of established murine colon carcinoma metastases by antibody-interleukin 2 fusion protein therapy. Cancer research 57:4948-4955.

41. Johnson, E.E., Yamane, B.H., Buhtoiarov, I.N., Lum, H.D., Rakhmilevich, A.L., Mahvi, D.M., Gillies, S.D., and Sondel, P.M. 2009. Radiofrequency ablation combined with KS-IL2 immunocytokine (EMD 273066) results in an enhanced antitumor effect against murine colon adenocarcinoma. Clinical cancer research : an official journal of the American Association for Cancer Research 15:4875-4884.

42. Gutbrodt, K.L., Schliemann, C., Giovannoni, L., Frey, K., Pabst, T., Klapper, W., Berdel, W.E., and Neri, D. 2013. Antibody-based delivery of interleukin-2 to neovasculature has potent activity against acute myeloid leukemia. Science translational medicine 5:201ra118.

43. Hemmerle, T., and Neri, D. 2014. The antibody-based targeted delivery of interleukin-4 and 12 to the tumor neovasculature eradicates tumors in three mouse models of cancer. International journal of cancer. Journal international du cancer 134:467-477.

44. Apetoh, L., Ghiringhelli, F., Tesniere, A., Obeid, M., Ortiz, C., Criollo, A., Mignot, G., Maiuri, M.C., Ullrich, E., Saulnier, P., et al. 2007. Toll-like receptor 4-dependent contribution of the immune system to anticancer chemotherapy and radiotherapy. Nature medicine 13:1050-1059.

45. Seung, S.K., Curti, B.D., Crittenden, M., Walker, E., Coffey, T., Siebert, J.C., Miller, W., Payne, R., Glenn, L., Bageac, A., et al. 2012. Phase 1 study of stereotactic body radiotherapy and interleukin-2--tumor and immunological responses. Science translational medicine 4:137ra174.

46. Pfaffen, S., Frey, K., Stutz, I., Roesli, C., and Neri, D. 2010. Tumour-targeting properties of antibodies specific to MMP-1A, MMP-2 and MMP-3. European journal of nuclear medicine and molecular imaging 37:1559-1565.

47. Edelstein, A., Amodaj, N., Hoover, K., Vale, R., and Stuurman, N. 2010. Computer control of microscopes using microManager. Current protocols in molecular biology / edited by Frederick M. Ausubel ... [et al.] Chapter 14:Unit14 20. 


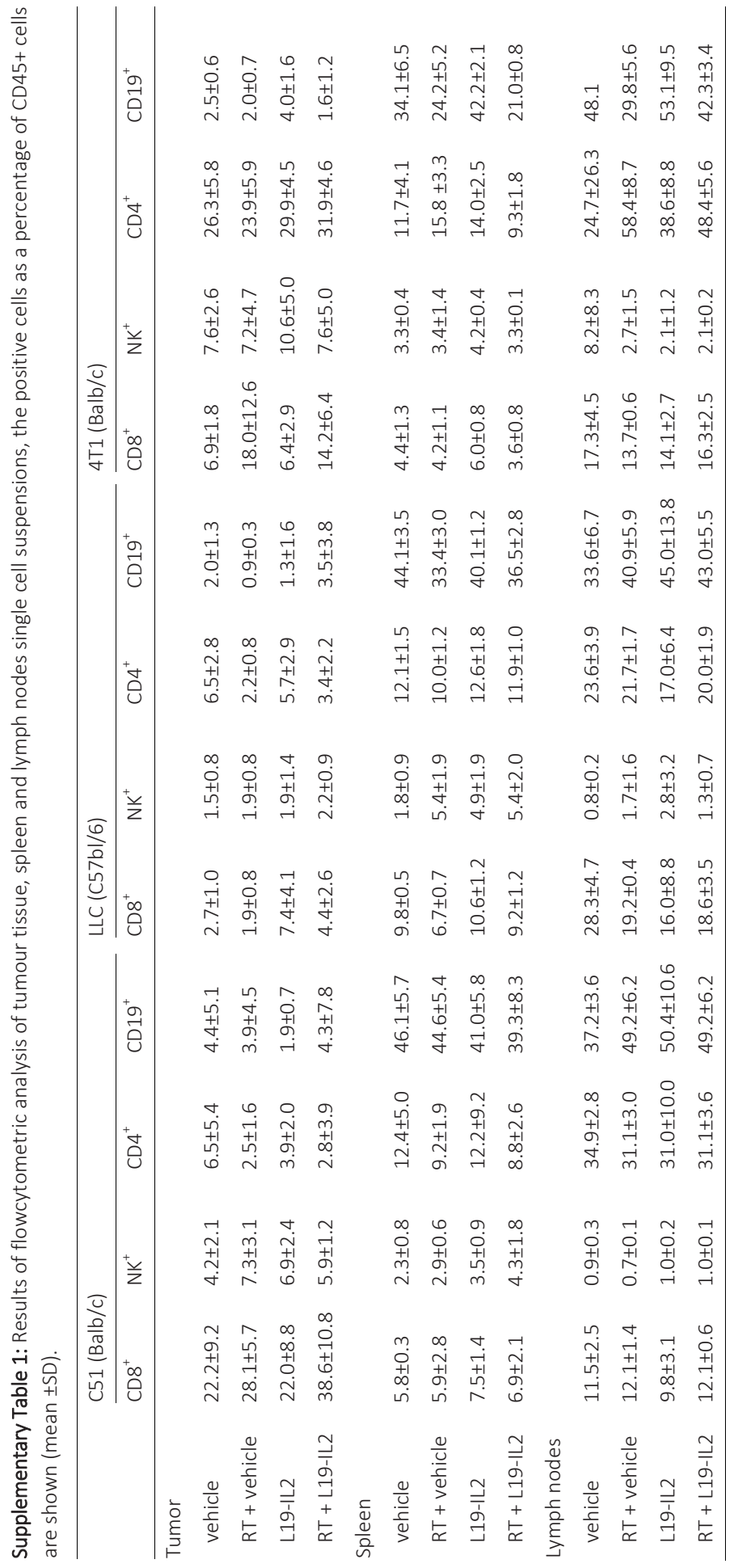


Radiotherapy combined with the immunocytokine L19-IL2 provides long-lasting anti-

tumour effects

A

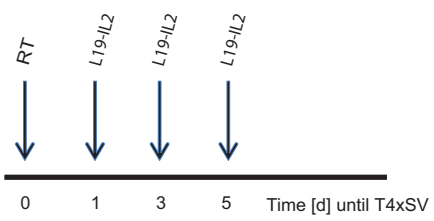

B

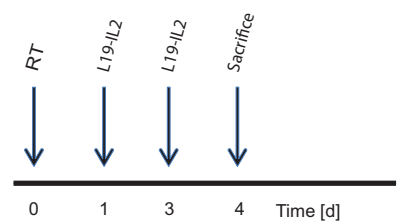

Supplementary figure 1: Treatment schedules used in in-vivo experiments. A: Schedule used for follow-up of 4 times starting volume of the tumours. B: Schedule used for flow cytometry and fluorescent stainings, mice are sacrificed at day 4 .
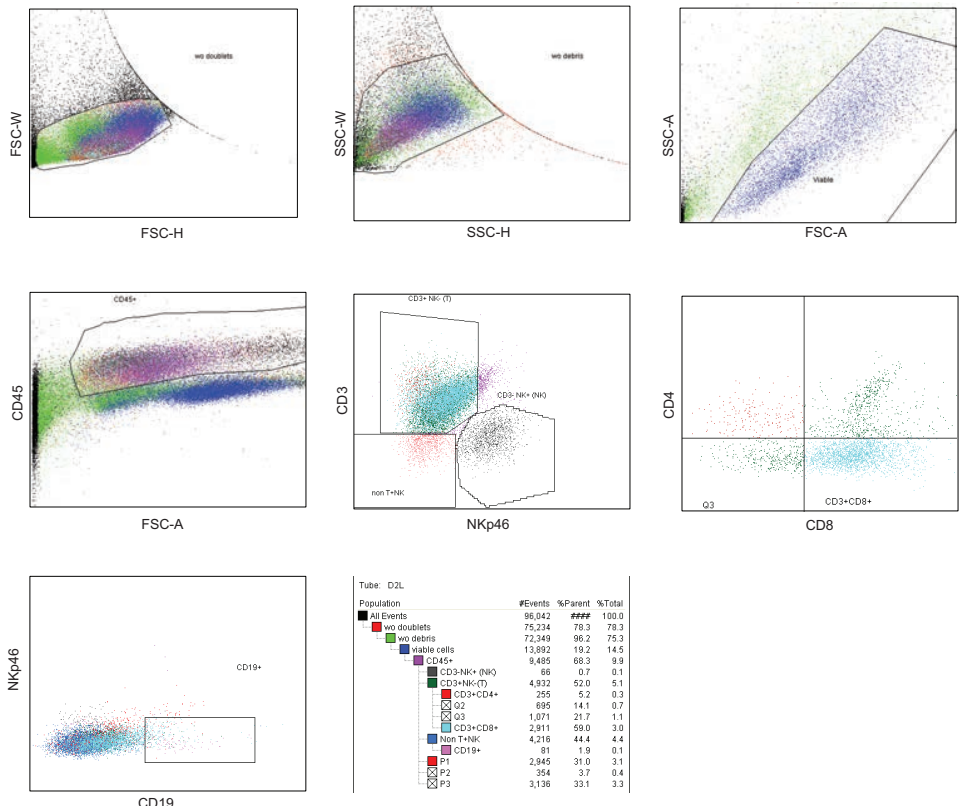

Supplementary figure 2: FACS gating strategy (tumour). Gating strategy used for flow cytometry analysis of tumors, lymph nodes and spleens, shown is the gating for a tumour. 


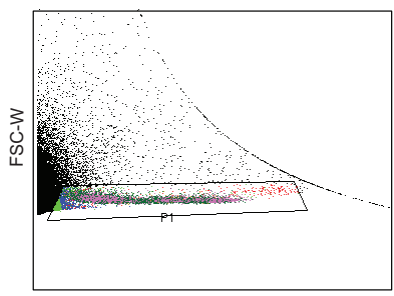

FSC-H

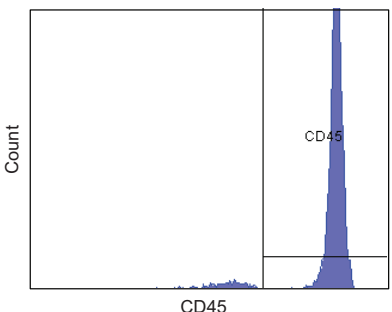

CD45

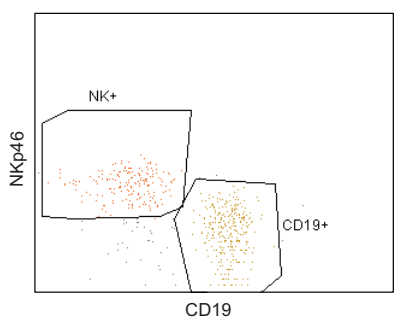

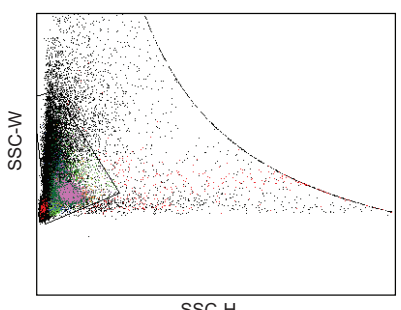

SSC-H

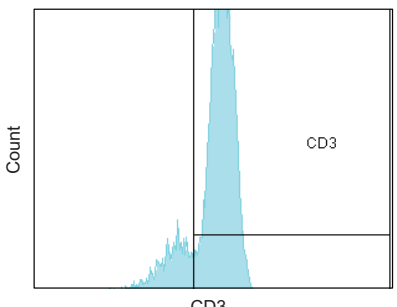

CD3

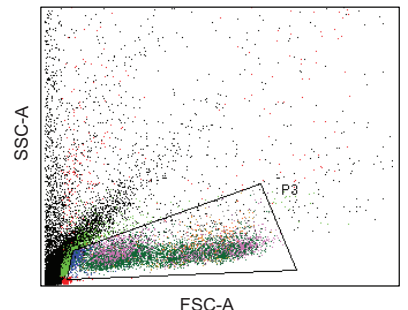

FSC-A

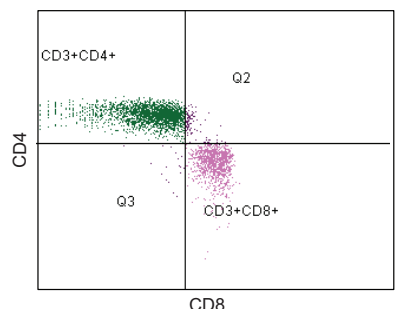

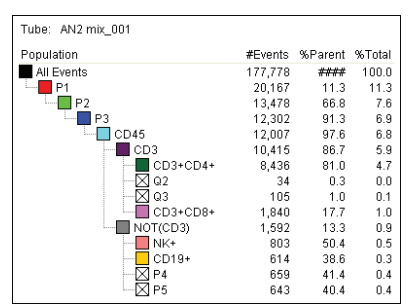

Supplementary figure 3: FACS gating strategy (blood). Gating strategy used for flow cytometry analysis of blood in CD8 depletion experiment. 
Radiotherapy combined with the immunocytokine L19-IL2 provides long-lasting anti-

tumour effects

A

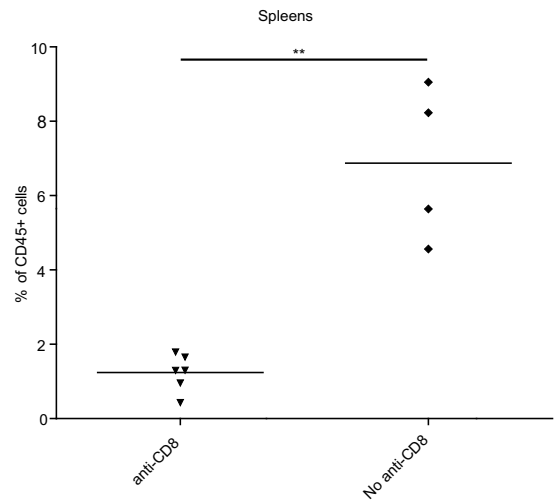

Blood

B

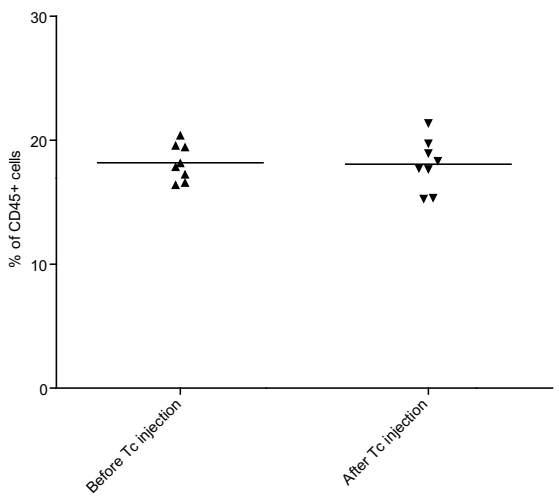

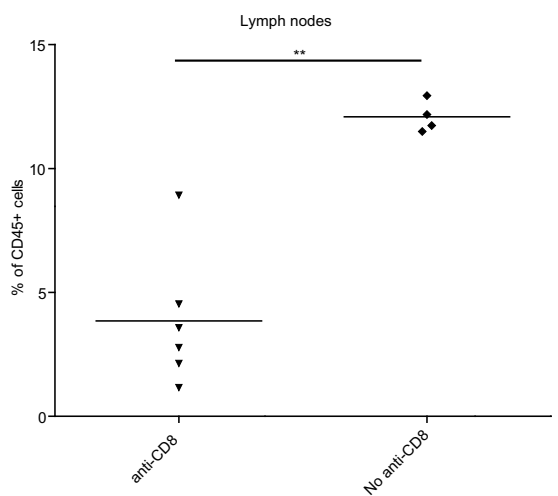

Supplementary figure 4: Cytotoxic T cells $\left(C D 8^{+}\right)$as a $\%$ of $C D 45^{+}$cells. A: \% of $C D 8^{+}$T cells of $C D 45^{+}$cells in spleens or lymph nodes after CD8 depletion (anti-CD8 i.p.) compared with control mice (tumor bearing mice, treated with RT+L19-IL2 without CD8 depletion). Data are analyzed with flow cytometry. B: $\%$ of CD ${ }^{+} \mathrm{T}$ cells of $\mathrm{CD}_{4} 5^{+}$cells in the blood 6 days before and 1 day after tumor cell (Tc) injection. ${ }^{*} p<0.01$. 



\section{Chapter}

\section{Long-lasting anti-tumour effects provided by Radiotherapy combined with the immunocytokine L19-IL2}

Nicolle H. Rekers ${ }^{*}$, Catharina M.L. Zegers, ${ }^{*}$ Wilfred T.V. Germeraad, Ludwig Dubois ${ }^{* *}$, Philippe Lambin ${ }^{* *}$

Published in: Oncolmmunology. 2015; 4(8):e1021541 


\section{ABSTRACT}

Recently, we have shown that radiotherapy combined with L19-IL2 can induce a longlasting anti-tumour effect, dependent on ED-B expression and infiltration of cytotoxic T cells. These findings will be translated to a Phase I clinical study (NCT02086721) in patients with oligometastatic solid tumours. See this link for the animation:

http://youtu.be/xHbwQuCTkRc. 


\section{AUTO-COMMENTARY}

Radiotherapy (RT) has several effects in the tumour micro-environment, it causes the release of a broad range of tumour-associated antigens and damage molecular patterns (DAMPs) and upregulates immunomodulatory cell surface and secretory molecules (1$3)$. These changes can increase the immunogenicity of the tumour and promote the uptake of tumour antigens by antigen presenting cells that cross-present these tumour antigens to $T$ cells, thereby triggering a cytotoxic T-lymphocyte response resulting in immunogenic tumour cell death $(1,3,4)$. Unfortunately, this systemic immune response against tumour cells provoked by local tumour RT alone is mostly insufficient to totally eradicate all tumour cells. Therefore, the addition of active immunotherapy (IT) to enhance the effect of RT may be an interesting approach to enhance the therapeutic potential (5).

The administration of IL2 as immunotherapy has the ability to stimulate and expand functional active $T$ cells which has been shown to lead to durable and curative regressions in patients with metastatic melanoma and renal cancer (6). However, a major drawback of this immunotherapy is the occurrence of several toxicities (e.g. capillary leakage syndrome, severe flu-like symptoms and coma) and therefore targeted delivery of IL2 to the tumour could be used to overcome these side effects. The selective delivery of IL2 via the small-immuno-protein L19 to the ED-B domain, a part of the fibronectin of the tumour neo-vasculature, has shown to have excellent tumour targeting properties and an improved therapeutic index over IL2 treatment alone (7). Based on the known immunogenic effects of RT on tumours, the immune stimulating effects of IL2 and the possibility of targeted delivery via the immunocytokine L19-IL2, we recently demonstrated that a combination of RT with L19-IL2 causes an enhanced anti-tumour effect, dependent on ED-B expression (8).

In our preclinical study, we used three immunocompetent mouse tumour models; a colon carcinoma (C51), the Lewis Lung Carcinoma (LLC) and a breast carcinoma (4T1) model showing respectively high, intermediate and low ED-B expression levels. Combination of RT with L19-IL2 resulted in long-lasting, highly synergistic effects with a cure rate of $75 \%$ in the high ED-B expressing model C51. Lowering the RT dose to 5 and 2 Gy decreased the cure rate of the combination therapy to $50 \%$ and $8 \%$, respectively. In the LLC model, we observed an additive effect when a single RT dose of $10 \mathrm{~Gy}$ was combined with L19-IL2 compared to single treatment arms. In addition, the expression of ED-B seemed to be crucial, since the combination treatment showed no additional therapeutic improvement in the low ED-B expressing model 4T1. Further investigation of tumour immune infiltrate showed a significant increase of CD8+ cytotoxic T cells in C51 and LLC tumours treated with RT and L19-IL2, suggestion a pivotal role for these immune cells. Indeed, when depleting CD8+ cells in vivo using the same preclinical experimental setting as in the C51 model, no benefit of the combination therapy could 
be observed, providing evidence that the complete remission of these C51 tumours is attributed to the presence and activity of cytotoxic T cells (8). Taken together, we have shown that combination therapy of RT with L19-IL2 can enhance the RT induced antitumour immune reaction, providing a long-lasting anti-tumour effect when ED-B is present. Since L19-IL2 is proven to be safe in patients, these findings will be translated to a Phase I clinical study (NCT02086721) in patients with oligometastatic solid tumours.

The previous results provide a basis to understand the anti-tumour immune response after the combination therapy. However, it also provides potential new mechanisms to investigate in pre-clinical setting. One interesting mechanism is the abscopal effect, an anti-tumour effect observed in tumour cells located outside the radiation field, possibly mediated by the immune system (9). We hypothesize that the immune system is an important mediator of the abscopal effect, and therefore therapy of RT with L19-IL2 may be an interesting combination to induce and investigate this phenomenon. After $\mathrm{RT}$, a broad variation of tumour-associated antigens is systemically released to function as an 'in situ vaccination' and therefore DC uptake of this antigen mixture might be responsible for the stimulation of a broad cytotoxic T-cell mediated anti-tumour response, recognizing several antigens expressed on the heterogenic tumour cell population. This can be beneficial in the treatment of multiple tumours and metastases, in and outside the radiation field. Furthermore, an important feature of the adaptive immune system is its memory, which leads to an enhanced response when reencountering the same antigen. It will be of major interest to investigate if cytotoxic Tcells triggered by RT+L19-IL2 treatment are indeed able to recognize the same tumourassociated antigens and kill the tumour cells after cure. In other words: do these cytotoxic T-cells have a memory potential? The proliferation of cytotoxic T-cells at the tumour site is of major importance for the development of a strong enough antitumour immune response. However, it is known that hypoxic regions inside solid tumoirs can prevent an adequate anti-tumour immune response (10), possibly explained by the lack of infiltration of cytotoxic T-cells inside the hypoxic areas of treated tumours, suggesting that the combination of this novel treatment (RT+L19-IL2) with anti-hypoxia therapy might be successful.

In conclusion, we have shown a cytotoxic T-cell response after RT+L19-IL2 leads to cure in the large majority of treated mice in the C51 model and new research should therefore focus on the cellular and molecular mechanisms underlying this effect as well as on combination treatment which can break the hypoxia immuno-sanctuary. 

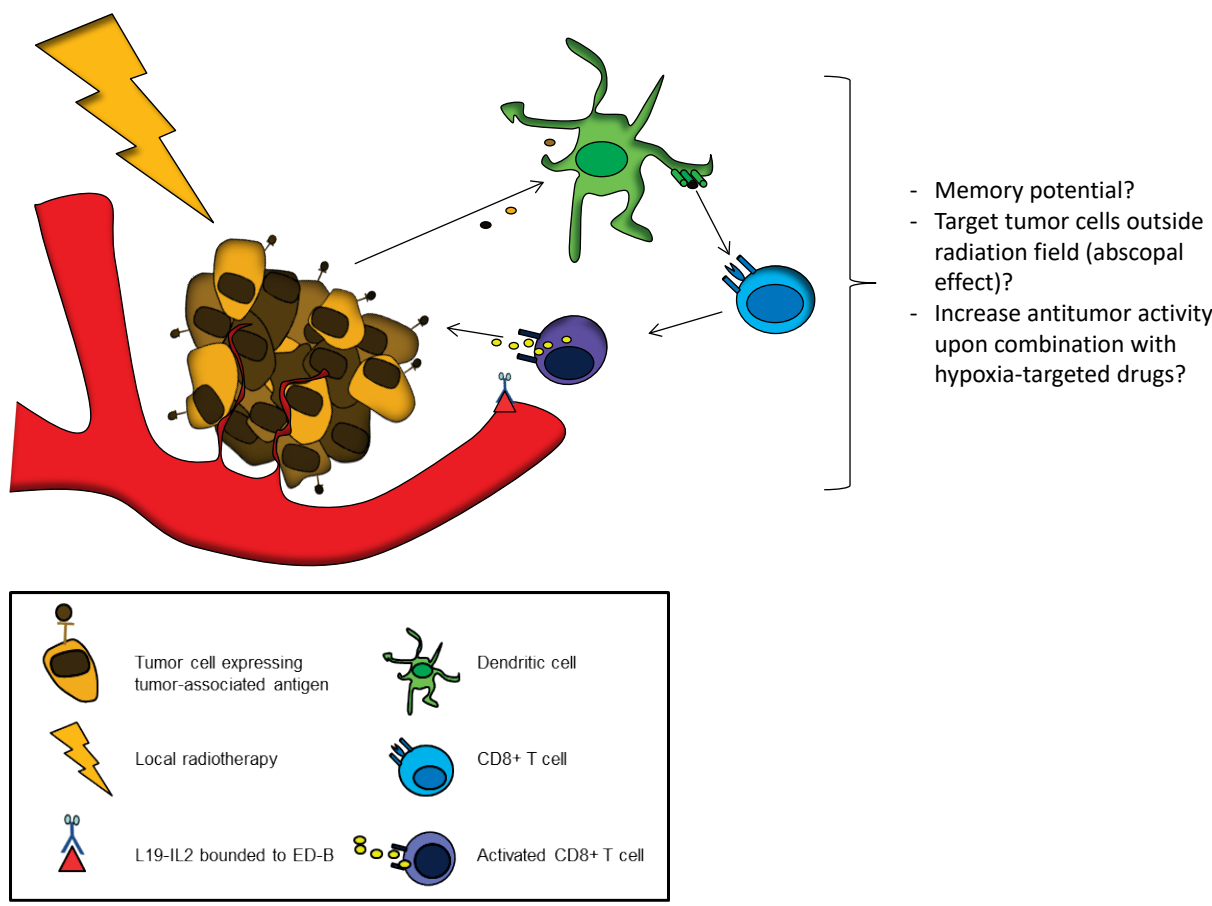

Figure 1: Radiotherapy causes a synergistic and long-lasting anti-tumour immune response when combined with L19-IL2. This response is dependent on the presence of ED-B in tumour blood vessels and on the presence of cytotoxic T cells inside the tumour. 


\section{REFERENCES}

1. Demaria S, Formenti SC. Radiation as an immunological adjuvant: current evidence on dose and fractionation. Front Oncol 2012; 2:153.

2. Kaur P, Asea A. Radiation-induced effects and the immune system in cancer. Frontiers in oncology 2012; 2:191.

3. Krysko DV, Garg AD, Kaczmarek A, Krysko O, Agostinis P, Vandenabeele P. Immunogenic cell death and DAMPs in cancer therapy. Nature reviews Cancer 2012; 12:860-75.

4. Green DR, Ferguson T, Zitvogel L, Kroemer G. Immunogenic and tolerogenic cell death. Nat Rev Immunol 2009; 9:353-63.

5. Rekers NH, Troost EG, Zegers CM, Germeraad WT, Dubois LJ, Lambin P. Stereotactic ablative body radiotherapy combined with immunotherapy: present status and future perspectives. Cancer Radiother 2014; 18:391-5.

6. Rosenberg SA. IL-2: the first effective immunotherapy for human cancer. Journal of immunology 2014; 192:5451-8.

7. List T, Neri D. Immunocytokines: a review of molecules in clinical development for cancer therapy. Clin Pharmacol 2013; 5:29-45.

8. Zegers CM, Rekers NH, Quaden DH, Lieuwes NG, Yaromina A, Germeraad WT, Wieten L, Biessen EA, Boon L, Neri D, et al. Radiotherapy Combined with the Immunocytokine L19-IL2 Provides Long-lasting Antitumor Effects. Clin Cancer Res 2015; DOI: 10.1158/1078-0432.CCR-14-2676.

9. Postow MA, Callahan MK, Barker CA, Yamada Y, Yuan J, Kitano S, Mu Z, Rasalan T, Adamow M, Ritter E, et al. Immunologic correlates of the abscopal effect in a patient with melanoma. N Engl J Med 2012; 366:925-31.

10. Chouaib S, Messai Y, Couve S, Escudier B, Hasmim M, Noman MZ. Hypoxia promotes tumor growth in linking angiogenesis to immune escape. Frontiers in immunology 2012; 3:21. 
Chapter

\section{The immunocytokine L19-IL2: a perfect interplay between radiotherapy and long-lasting systemic anti-tumour immune responses}

Nicolle H. Rekers*, Veronica Olivo Pimentel*, Ala Yaromina, Natasja G. Lieuwes, Rianne Biemans, Catharina M.L. Zegers, Wilfred T.V. Germeraad, Evert J. Van Limbergen, Dario Neri, Ludwig J. Dubois**, Philippe Lambin**

Submitted to: Nature Communications 


\section{ABSTRACT}

We recently have shown that the administration of the tumour-targeted immunocytokine L19-IL2 after radiotherapy (RT) resulted in synergistic anti-tumour effects. Here we tested the hypothesis that RT and L19-IL2 can activate a curative out-of-field radioimmune (OFRI) effect, with a long-lasting immunological memory. Ionizing radiation (15Gy, 5x2Gy or $5 \times 5 \mathrm{~Gy}$ ) was delivered to primary C51 colon tumour-bearing immunocompetent mice in combination with L19-IL2 and therapeutic efficacy was assessed on the secondary C51 or CT26 colon tumours. We show, for the first time, that only 15Gy + L19-IL2 can trigger a curative OFRI effect, which is T cell dependent. Moreover only RT + L19-IL2, and not surgery + L19-IL2 or high dose RT alone, results in long-lasting immunological memory, associated with $\mathrm{CD}_{4} 4^{+} \mathrm{CD} 127^{+}$expression on circulating $\mathrm{T}$ cells. This combination treatment can induce highly specific and long-lasting curative OFRI responses, and therefore it has great potential for treatment of (oligo)metastatic disease (https://youtu.be/aOeJXn7wNVQ). 


\section{INTRODUCTION}

Radiotherapy (RT) is known to enhance the release of a broad range of tumourassociated antigens and damage associated molecular patterns (DAMPs). It also stimulates the upregulation of immunomodulatory cell surface molecules. Together this results in the creation of a personified 'in situ vaccine' thereby initiating an immune response (1-3). These changes in tumour immunogenicity promote the uptake of tumour antigens by antigen presenting cells that cross-present the tumour antigens to $T$ cells, thereby triggering a cytotoxic T-lymphocyte response (1, 3-5). In some cases, tumour regression outside the radiation field can be observed, a phenomenon known as the abscopal effect (6-10). Although the principle of the abscopal effect is intriguing, its clinical appearance is sporadic (11). It has been established that the RT-induced abscopal effect is immune mediated (12-17) and therefore the rationale of combining RT with immunotherapeutic approaches to further increase systemic anti-tumour effects has recently gained a lot of interest (18).

The cytokine interleukin 2 (IL2) stimulates the proliferation and differentiation of cytotoxic, helper and regulatory T cells, and natural killer (NK) cells, resulting in a balanced pro- and anti-inflammatory immune response (19). IL2 treatment has shown durable and curative regressions in patients with metastatic melanoma, renal cancer and advanced non-Hodgkin's lymphomas and represents the first effective immunotherapy $(20,21)$. However, further clinical systemic use has been hampered due to the appearance of several severe toxicities (e.g. capillary leakage syndrome, severe flu-like symptoms, and coma) (22). Specific targeting of IL2 to the tumour using the vehicle L19, an antibody fragment directed against the angiogenesis-associated Bfibronectin isoform (ED-B) typically overexpressed in solid tumours, resulted in achieving high intra-tumoural IL2 doses without increasing toxicity (22). The immunocytokine L19-IL2 has been investigated in a randomized phase II clinical trial in patients with metastatic melanoma in combination with dacarbazine or with L19-TNF $\alpha$ (tumour necrosis factor) showing encouraging anti-tumour activity (23-27). Furthermore, tumour-specific (neo)antigens are important recognition sites for immune cells $(28,29)$ and since RT increases the tumour (neo)antigen expression it has great immunotherapeutic improving potential for all solid tumours (5).

Recently, we have demonstrated that the combination of a single RT dose with L19-IL2 resulted in long-lasting, highly synergistic anti-tumour effects with a cure rate of $75 \%$ in the high ED-B expressing C51 mouse colon carcinoma. Expression of ED-B by the tumour vasculature as well as infiltration of $\mathrm{CD} 8^{+} \mathrm{T}$ cells was crucial for this pronounced anti-tumour immune response (30). Similar to the C51 tumour model, ED-B is overexpressed in the majority of solid tumours (31-35), making this combination therapy of great interest. These highly synergistic preclinical findings resulted in the 
initiation of a phase I clinical study in our institute (NCT02086721), in which stereotactic radiotherapy is combined with L19-IL2 in patients with oligometastatic tumours.

Our ultimate goal is to increase overall survival in patients with systemic (metastatic) cancer. However, in this context, RT is assumed to be of limited use as it is difficult to apply in these patients: first due to wide spread tumour burden and secondly due to the fact that RT cannot be directed towards invisible (micro)metastases. In the current study, we have investigated for the first time the systemic and long-lasting anti-tumour effects elicited by RT combined with L19-IL2, providing us a unique insight into their synergistic interplay and induced immune responses. Since we have shown that the synergistic effect of RT + L19-IL2 against irradiated tumours is highly dependent on the immune system, we hypothesize that this combination therapy can elicit Out-of-Field Radiolmmune (OFRI) effects targeting macroscopic tumours. This study provides us with exclusive knowledge about the OFRI effect, comparing fractionated and single dose RT, irradiated and non-irradiated tumours, responding and non-responding mice and differences in potential to induce long-lasting protection (memory effect). The latter is of great importance, since most conventional treatment options only work during and shortly after their administration, i.e. have transient effect. These in vivo data reveal the possibility to create a long-lasting OFRI effect and form the basis of a preclinical treatment recommendation towards curative treatment of systemic cancer. 


\section{RESULTS}

\section{A single RT dose combined with L19-IL2 triggers a curative OFRI effect}

Previously, we have shown that a single RT dose of $10 \mathrm{~Gy}$ resulted in $75 \%$ cure when combined with L19-IL2 in the high ED-B expressing C51 model, while 5Gy and 2Gy led to a cure rate of $50 \%$ and $8 \%$, respectively(30). To test whether RT to the primary tumour combined with L19-IL2 has an anti-tumour effect on non-irradiated secondary tumours, i.e. can induce an OFRI effect, we first determined which RT dose and schedule resulted in maximal anti-tumour activity (100\% cure) when combined with L19-IL2. Previously, we have shown that a single RT dose of $10 \mathrm{~Gy}$ resulted in $75 \%$ cure when combined with L19-IL2 in the high ED-B expressing C51 model, while 5Gy and 2Gy led to a cure rate of $50 \%$ and $8 \%$ respectively (30). Increasing the RT dose to $15 \mathrm{~Gy}$, resulted in $100 \%$ cure when combined with L19-IL2 (supplementary Fig. 1a), which was significantly higher than 15Gy + vehicle control (supplementary Fig. 1b). However, in clinical setting, RT is often given still in multiple fractions. Therefore, we additionally investigated L19-IL2 in combination with fractionated RT. Combining 5 fractions of 2Gy with L19-IL2 (days 1, 3, 5) resulted in $100 \%$ cure of the primary tumours (supplementary Fig. 1c).

Next, we investigated the therapeutic effect of 15Gy, $5 \times 2$ Gy and $5 \times 5 G y$ delivered to the primary tumour in combination with systemic L19-IL2 treatment on the nonirradiated secondary tumours (Fig. 1a). The extra fractionated RT treatment arm, $5 \mathrm{x}$ $5 G y$, was included because it is biologically equivalent to $15 G y$ single dose. Starting volumes of secondary tumours were similar across different treatment groups (supplementary table 1). Secondary tumours of mice treated with 15Gy + L19-IL2 showed a significant ( $p=0.03$ ) delay of median T4XSV (9.6 [4.3-26.4] days) as compared with 15Gy + vehicle (5.7 [5.1-8.9] days) with a cure rate of $20 \%(2 / 10)$ (Fig. 1b). Addition of L19-IL2 to $5 \times 2$ Gy significantly $(p=0.003)$ increased T4xSV of the non-irradiated tumours from 5.9 [3.1-7.7] days to 8.8 [5.7-17.6] days, to a similar extent as $5 \times 5 G y+$ L19-IL2 (T4XSV of 10.1 [6.6-12.9] days). In contrast to 15Gy, none of the secondary tumours of the fractionated RT groups could be cured (Fig. 1b), demonstrating that a single high radiation dose is a better trigger of RT + L19-IL2 mediated curative OFRI effect.

For a single 15Gy RT dose in combination with L19-IL2, we observed at day 4 a significantly $(p=0.02)$ higher percent of $\mathrm{CD}^{+} \mathrm{T}$ cells inside secondary tumours $(8.3 \%$ [2.9-42.3]) as compared with the primary tumours (2.0\% [0.2-3.4]) (Fig. 1c). In addition, the percentage of $\mathrm{CD}^{+} \mathrm{T}$ cells in 15Gy + L19-IL2 treated tumours is significantly lower as compared with L19-IL2 treated tumours (4.1\% [1.2-5.2], p=0.02). At this time point, no involvement of infiltrating $\mathrm{CD}^{+}$cytotoxic T cells could be observed (Fig. 1C). 
A

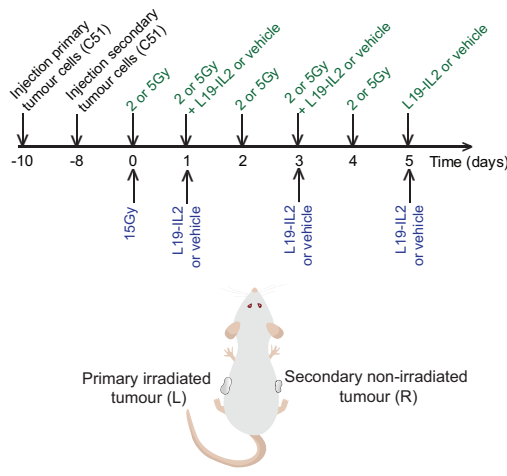

B

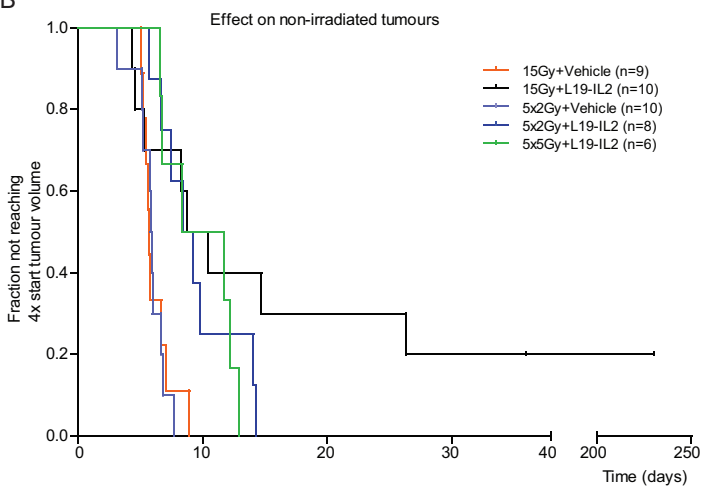

C Intratumoural immune cells day 4
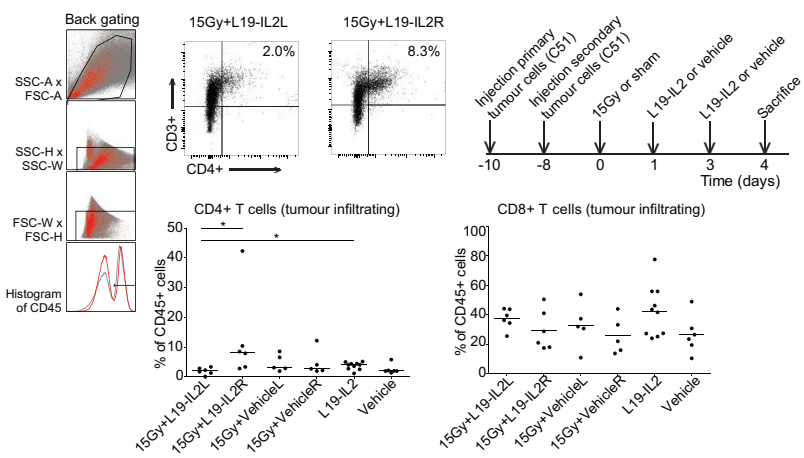

CD8+ T cells (tumour infiltrating)

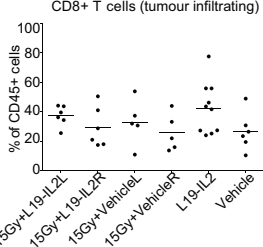

Figure 1: Radiotherapy and L19-IL2 induced OFRI effects and associated immune infiltrate. (A) Experimental setup and schedule used for OFRI study. (B) The fraction of non-irradiated tumours not reaching 4 times start tumour volume. (C) Representative back gating (red), FACS image showing intratumoural $\mathrm{CD}^{+} \mathrm{CD} 4^{+}$cells of gated $\mathrm{CD} 45^{+}$cells of irradiated $(\mathrm{L})$ and non-irradiated $(\mathrm{R})$ tumours, a quantification of the intratumoural $\mathrm{CD}^{+} \mathrm{CD}^{+}$and $\mathrm{CD}^{+} \mathrm{CD}^{+}$cells and the experimental schedule used. Data represent the median. ${ }^{*} \mathrm{p}<0.05$.

The curative OFRI effect is dependent on $C D 4^{+}$and $C D 8^{+} T$ cells and is tumour cell specific

To assess the causal relationship between the anti-tumour OFRI effect and CD4+ T cells, we depleted the CD4+ T cells 5 days after RT (Fig. 2a, supplementary table 1). In addition, in a similar manner, the contribution of CD8+ T cells to the OFRI effect was evaluated. CD8+ and CD4+ T cells were successfully depleted 2 days after the first i.p. injection to (0.2\% [0-0.6]) and $0 \%$ as compared to IgG control (9.2\% [6.2-13.7, $p=0.003])$ and $(36.0 \%$ [19.2-52.5, $\mathrm{p}=0.003]$ ), respectively (Fig. 2a). Depletion of CD8+ or CD4+ T cells had little to no effect on the cure rate of irradiated primary tumours (supplementary Fig. 2a). These observations suggest that the immunological activity against the primary tumour has already been completed and it is likely that the OFRI effect has already been initiated at the time point when depleting antibodies were 
administered. Mice were randomized among the three depletion groups (supplementary Fig. 2c, supplementary table 1): CD8+ T cell, CD4+ T cell depleting antibodies or IgG control (Fig. 2a). IgG treatment resulted in a 29\% (2/7) cure rate of secondary non-irradiated tumours (Fig. 2b) consistent with the results obtained in the previous independent experiment (Fig. 1b). Tumour response was not changed upon CD4+ T cell depletion as compared to IgG control ( $p=0.21$ ) (Fig. 2b). Depletion of CD8+ T cells resulted in significantly $(p=0.01)$ faster growth of secondary tumours as compared to IgG control, demonstrating a causal role for cytotoxic T cells in the RT + L19-IL2 induced OFRI effect.

Next, we investigated the specificity of the OFRI anti-tumour effect, using the ED-B positive CT26 tumour (36) as a non-irradiated secondary tumour (Fig. 2c and supplementary table 1). In contrast to the matched C51 secondary tumours, which showed a significant growth delay when mice were treated with 15Gy + L19-IL2 as compared with 15Gy + vehicle (Fig. 1b), the growth delay of mismatched CT26 secondary tumours was not significantly $(p=0.2)$ different: T4XSV= 14.1 [5.5-50.7] days in 15Gy + L19-IL2 treated vs 9.0 [6.5-12.9] days in 15Gy + vehicle treated mice. In addition, growth delay was not significantly different between the secondary C51 and CT26 tumours when mice were treated with 15Gy + L19-IL2 ( $p=0.9)$, though no tumour cures could be observed in the latter group (Fig. $2 \mathrm{~d}$ ).

\section{A single RT dose combats the intratumoural immunosuppressive T cell phenotype}

We have shown that a single 15Gy RT dose + L19-IL2 induced a curative OFRI effect in only $20-30 \%$ of the non-irradiated tumours. We hypothesized that the same immune response triggered by this treatment combination is immunosuppressive or exhausted in non-irradiated tumours. Based on our findings and recent developments in FDA (Food and Drug Administration) approved therapies (37), we ought to investigate the expression of Foxp3 (an intracellular marker for regulatory $T$ cells) and the targetable immunosuppressive checkpoint receptors CTLA-4 and PD-1, on peripheral and intratumoural T cells. 

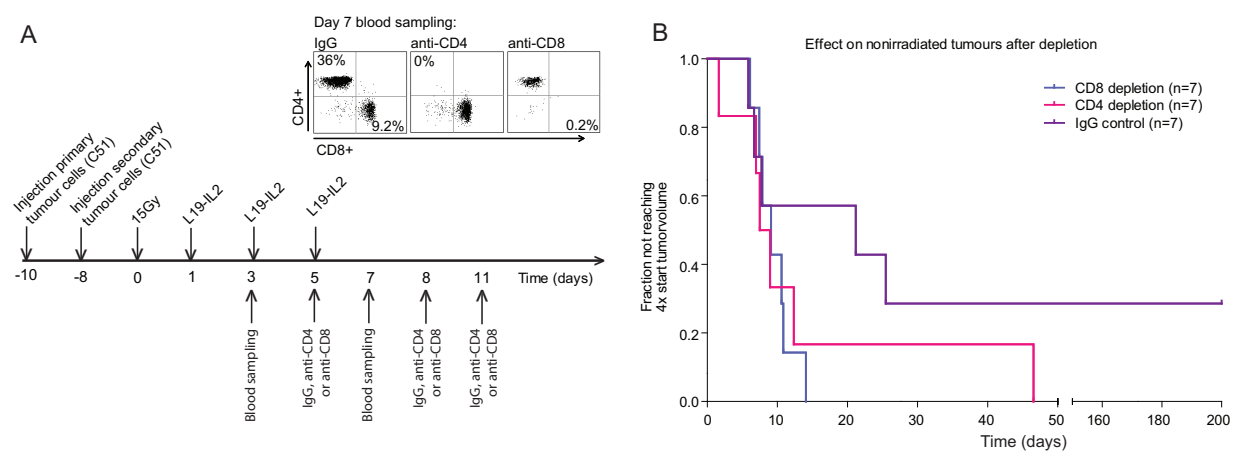

C

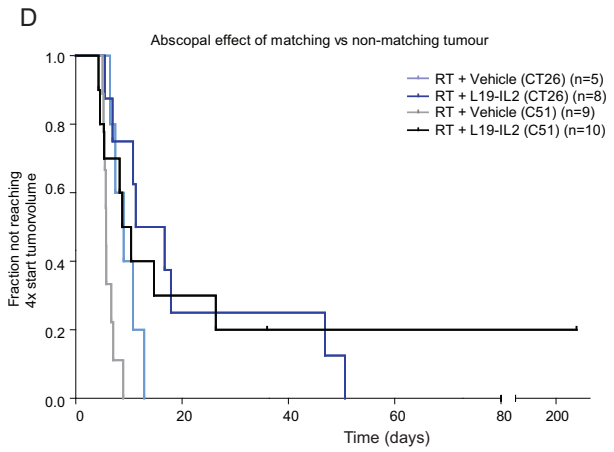

Figure 2: The OFRI effect in immune depleted and mismatched tumour-bearing mice. (A) Depletion study schedule and confirmation of depletion in blood analyzed with FACS. (B) Fraction of tumours not reaching 4 times start tumour volume. (C) Mismatch treatment schedule. (D) Fraction of tumours not reaching 4 times start tumour volume for the different treatment groups.

Although non-irradiated tumours from mice treated with 15Gy + L19-IL2 did not show higher PD-1 (Fig. 3a) or CTLA-4 (data not shown) expression on their CD8 ${ }^{+} \mathrm{T}$ cells as compared to irradiated tumours, the non-irradiated secondary tumour infiltrating CD4 ${ }^{+}$ T cells expressed significantly $(p=0.002)$ more PD-1 (4.2\% [1.7-6.7]) as compared with irradiated primary tumours $(0.8 \%$ [0.1-1.6]) and vehicle control (0.9\% [0.8-5.0], $p=0.02)$ (Fig. 3b). Furthermore, the non-irradiated tumours have a higher $\mathrm{CD} 25^{+} \mathrm{FoxP} 3^{+} \mathrm{CD} 4^{+} \mathrm{T}$ cell infiltrate $(1.8 \%$ [0.4-6.6], $p=0.009)$ as compared with the irradiated tumours $(0.2 \%$ [0.04-1.3]) (Fig. 3b). Strikingly, the immune infiltrate of irradiated tumours from mice treated with 15Gy + L19-IL2 showed a significantly lower percent PD- $1^{+}$expressing CD4 ${ }^{+}$ T cells $(0.8 \%,[0.1-1.6])$ in comparison with $15 \mathrm{~Gy}+$ vehicle $(2.0 \%$ [1.3-4.6], $p=0.01)$ and L19-IL2 (2.2\% [0.5-3.0], $p=0.005)$ treated tumours. A significantly $(p=0.01)$ lower percentage of Foxp $3^{+} \mathrm{CD} 25^{+}$expressing $\mathrm{CD}^{+} \mathrm{T}$ cells infiltrating irradiated tumours from RT + L19-IL2 treated mice (0.2\% [0.04-1.3]) as compared with L19-IL2 monotherapy (1.7\% [0.4-2.4]) was also observed. These data show that the addition of a single dose of 15Gy to the L19-IL2 monotherapy combats the immunosuppressive T cell infiltrate in irradiated tumours. Interestingly, this immunosuppressive combating potential could 
not be observed when combining L19-IL2 with fractionated RT (5 x 2Gy) (Fig. 3c). Although $\mathrm{CD}^{+}$and $\mathrm{CD}^{+} \mathrm{T}$ cell tumour infiltration was not significantly different between the irradiated and non-irradiated tumours (Fig 3d, e), addition of L19-IL2 to fractionated RT significantly increases PD- $1^{+}$expression on $\mathrm{CD}^{+}$infiltrating cells in irradiated tumours (2.0\% [1.2-2.7] $\mathrm{p}=0.01)$ as compared to the vehicle treated primary tumours (0.9\% [0.5-2.0]) (Fig. 3d). In addition, L19-IL2 increases PD- $1^{+}$expression on $\mathrm{CD}^{+}$infiltrating non-irradiated tumours (20.1\% [8.6-38.3], $\left.\mathrm{p}=0.01\right)$ as compared with 5 x 2Gy + vehicle treated non-irradiated tumours (7.5\% [4.0-8.9]) (Fig. 3e). No differences in peripheral $\mathrm{CD}^{+} \mathrm{T}$ cells were observed between all treatment groups (data not shown). In addition, no differences in peripheral $\mathrm{CD}^{+} \mathrm{T}$ cells were observed (supplementary Fig. 3a, b). Interestingly, fractionated RT did result in a higher expression of PD-1 on CD8 ${ }^{+} \mathrm{T}$ cells when combined with L19-IL2 (13.0 [1.8-25.1], $\mathrm{p}=0.03)$ as compared with the $5 \times 2 \mathrm{~Gy}+$ vehicle treated mice (1.2 [0.4-12.1]) (supplementary Fig. 3b). 
A
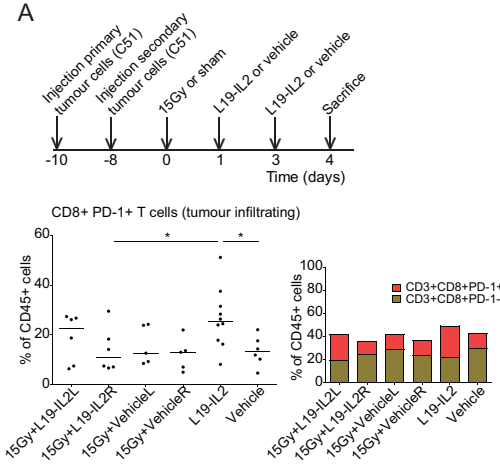

C

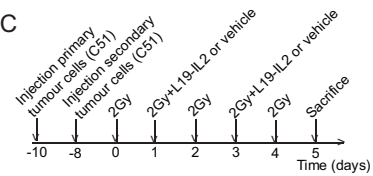

B

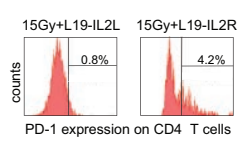

$\overbrace{\text { Foxp3+ }}^{15 \mathrm{~Gy}+\mathrm{L} 19-\mathrm{IL} 2 \mathrm{~L}}$
CD4+ PD-1+ T cells (tumour infiltrating)

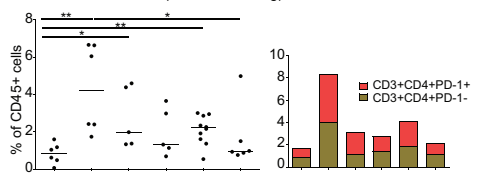

CD4+CD25+Foxp3+ T cells (tumour infiltrating)

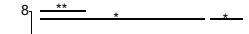

D

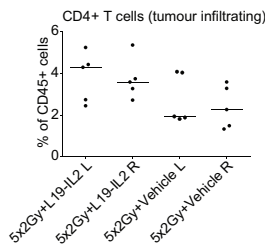

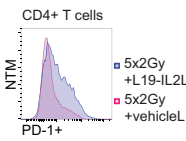

CD4+PD-1+T cells (tumour infiltrating)

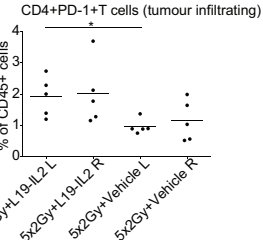

E

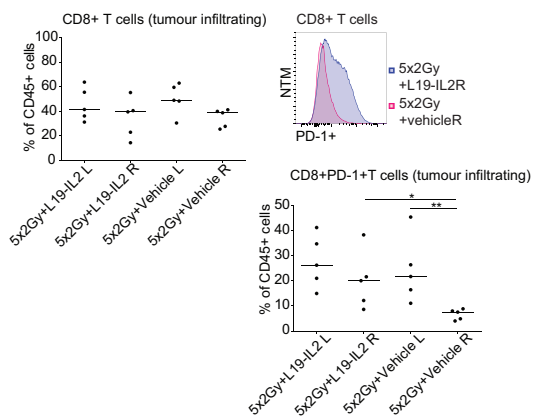

Figure 3: Co-expression of immune suppressive and exhausting receptors on $T$ cells. (A) Treatment schedule used for figure $A$ and $B, \%$ of intratumoural $C D 8^{+} P D-1^{+}$cells of the $C D 45^{+}$cells and a representation of the distribution (medians) of PD-1 $1^{+}$within the $C D 8^{+} \mathrm{T}$ cell population. (B) Representative FACS histogram and dotplot, the $\%$ of intratumoural $\mathrm{CD} 4^{+} \mathrm{PD}-1^{+}$cells and $\mathrm{CD} 4^{+} \mathrm{CD} 25^{+} \mathrm{Foxp} 3^{+} \mathrm{T}$ cells of $\mathrm{CD} 45^{+}$cells in different treatment groups and a representation of the distribution (medians) of PD- $1^{+}$and $C D 25^{+}$Foxp3 $3^{+}$expression within the $\mathrm{CD}^{+} \mathrm{T}$ cell population. (C) Fractionated treatment schedule used for figure $\mathrm{D}$ and $\mathrm{E}$. (D) Quantification of $C D 4^{+}$T cells of gated $C D 45^{+}$tumour infiltrating cells, FACS histogram of PD-1 expression on tumour infiltrating CD4+ T cells and a quantification of $C D 4^{+} \mathrm{PD}-1^{+}$cells. (E) Quantification of $\mathrm{CD} 8^{+} \mathrm{T}$ cells of gated $\mathrm{CD} 45^{+}$tumour infiltrating cells, FACS histogram of PD-1 expression on tumour infiltrating $\mathrm{CD} 8^{+} \mathrm{T}$ cells and a quantification of $\mathrm{CD} 8^{+}$PD $-1^{+}$cells. NTM: Normalized to Mode. ${ }^{*} p<0.05,{ }^{* *} p<0.01$. 
Single vs fractionated RT dose induced OFRI effect: more tumour infiltrating $\mathrm{CD}^{+}$and peripheral $\mathrm{CD} 4^{+} \mathrm{T}$ cells

To be able to compare single dose with the biologically equivalent fractionated RT + L19-IL2 OFRI effect, we investigated mice from 15Gy and $5 \times 5$ Gy combined with L19-IL2 treatment schedules at day 5 (Fig. 4a). Mice treated with $5 \times 5$ Gy + L19-IL2 had a significantly lower number of peripheral blood CD4+ T cells $(8.7 \%[5.5-12.4] \mathrm{p}=0.04)$ as compared with 15Gy + L19-IL2 treated mice (19.6 [6.2-29.2]) (Fig. 4b). No differences in $\mathrm{PD}-1^{+}, \mathrm{CTLA}-4^{+}$or FoxP3 ${ }^{+} \mathrm{CD} 25^{+}$T cells were found (Fig $4 . \mathrm{b}, \mathrm{c}$ ). At this time point, no differences in total number of tumour infiltrating $\mathrm{CD}^{+} \mathrm{T}$ cells could be observed (data not shown). The percentage of infiltrating $\mathrm{CD}^{+} \mathrm{T}$ cells however was significantly $(p=0.03)$ higher in the 15Gy + L19-IL2 $(49.3 \%$ [33.9 - 63]) as compared with $5 \times 5 G y+$ L19-IL2 (23.4\% [3.7 - 48.8]) non-irradiated tumours consistent with greater OFRI effect after the single dose regimen. The expression of PD- 1 and CTLA-4 on CD8 ${ }^{+}$T cells was not significantly different (Fig. 4c). 
A

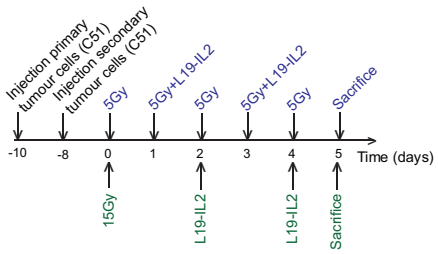

C

Intratumoural immune cells day 5

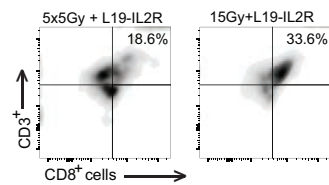

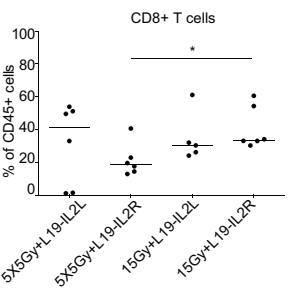

B
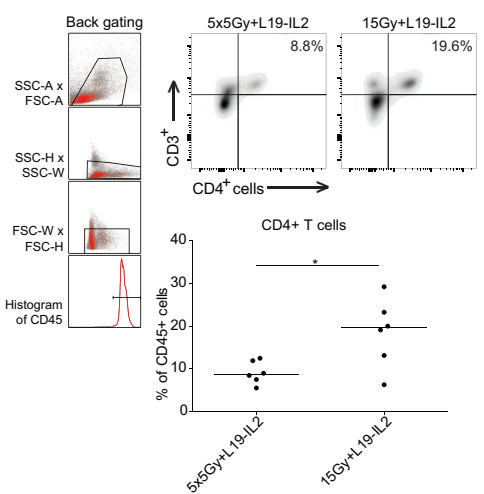

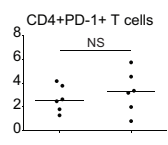

CD4+CTLA-4 T cells

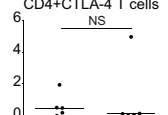

$\mathrm{CD} 4+\mathrm{CD} 25+\mathrm{FoxP} 3+\mathrm{T}$ cells 5 NS

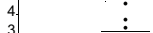
1. $\because \because \quad \vdots$

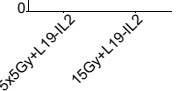

Figure 4: Comparison fractionated and single dose triggered OFRI anti-tumour response. (A) Experimental setup and schedule used for figure $B$ and $C$. (B) Representation and quantification of \% CD4 $4^{+}, C D 4^{+}$PD $-1^{+}, C D 4^{+}$ $\mathrm{CTLA}-4^{+}$and $\mathrm{CD}^{+} \mathrm{Foxp}^{+} \mathrm{CD} 25^{+} \mathrm{T}$ cells of total peripheral $\mathrm{CD} 45^{+}$immune cells. (C) Representation and quantification of $\% \mathrm{CD} 8^{+}, \mathrm{CD} 8^{+} \mathrm{PD}-1^{+}, \mathrm{CD} 8^{+} \mathrm{CTLA}-4^{+} \mathrm{T}$ cells of total tumour infiltrating $\mathrm{CD} 45^{+}$immune cells. $* p<0.05$.

\section{Long-lasting immunological memory associated with $C D 44^{+} C D 127^{+} C D 8^{+} T$ cells.}

Next we investigated if the anti-tumour immune response is modulated by the RT + L19IL2 treatment in such a manner that it remains active against its target, the tumourassociated (neo)antigens. To test this hypothesis, mice cured from C51 tumours by a single dose of 10Gy + L19-IL2 (30), where re-injected with C51 tumour cells 150 days after cure. While age-matched naive mice reached a tumour volume of $500 \mathrm{~mm}^{3}$ within 17 days after cell injection, cured mice did not show any tumour formation (Fig. 5a). Though not significant, FACS analyses at endpoint revealed a higher $\mathrm{CD} 44^{+} \mathrm{CD} 127^{+}$coexpression on $\mathrm{CD}^{+} \mathrm{T}$ cells in spleens (Fig. 5b) and lymph nodes (data not shown) in the re-challenged mice that were able to reject tumours (4.8 [0.9-6.4]) as compared with naive (0.9 [0.3-1.7]) and tumour-bearing (1.0 [0.8-1.8]) age-matched controls (Fig. 5b). Next, we investigated whether this long-lasting anti-tumour response solely depends on 
A

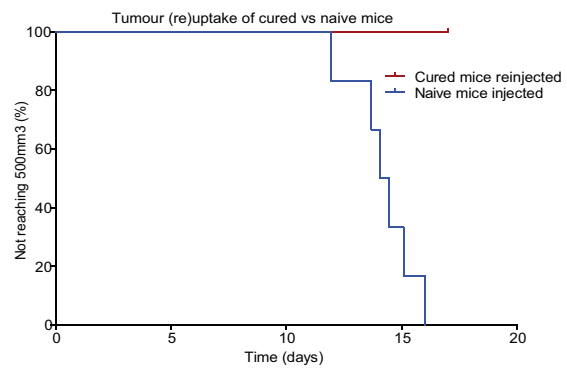

C

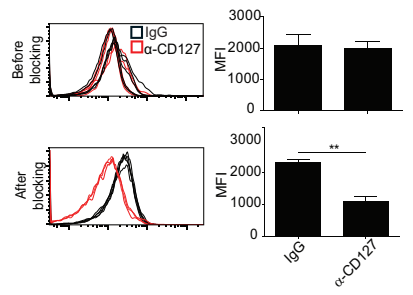

B

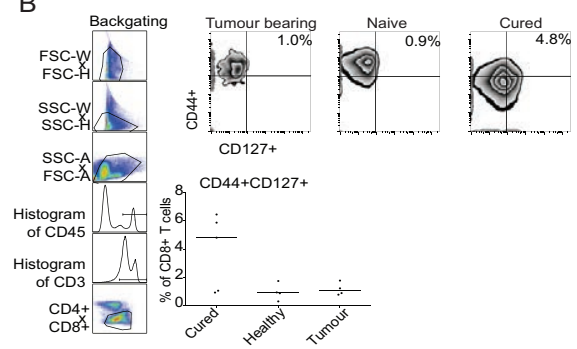

$\mathrm{D}$

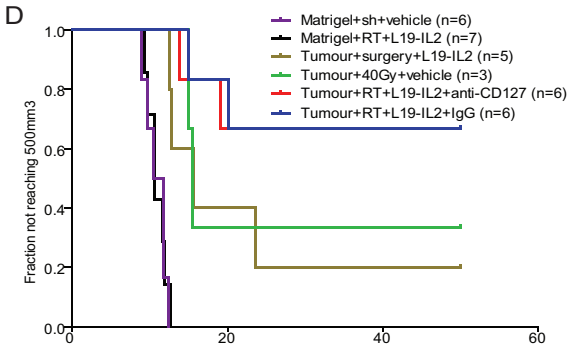

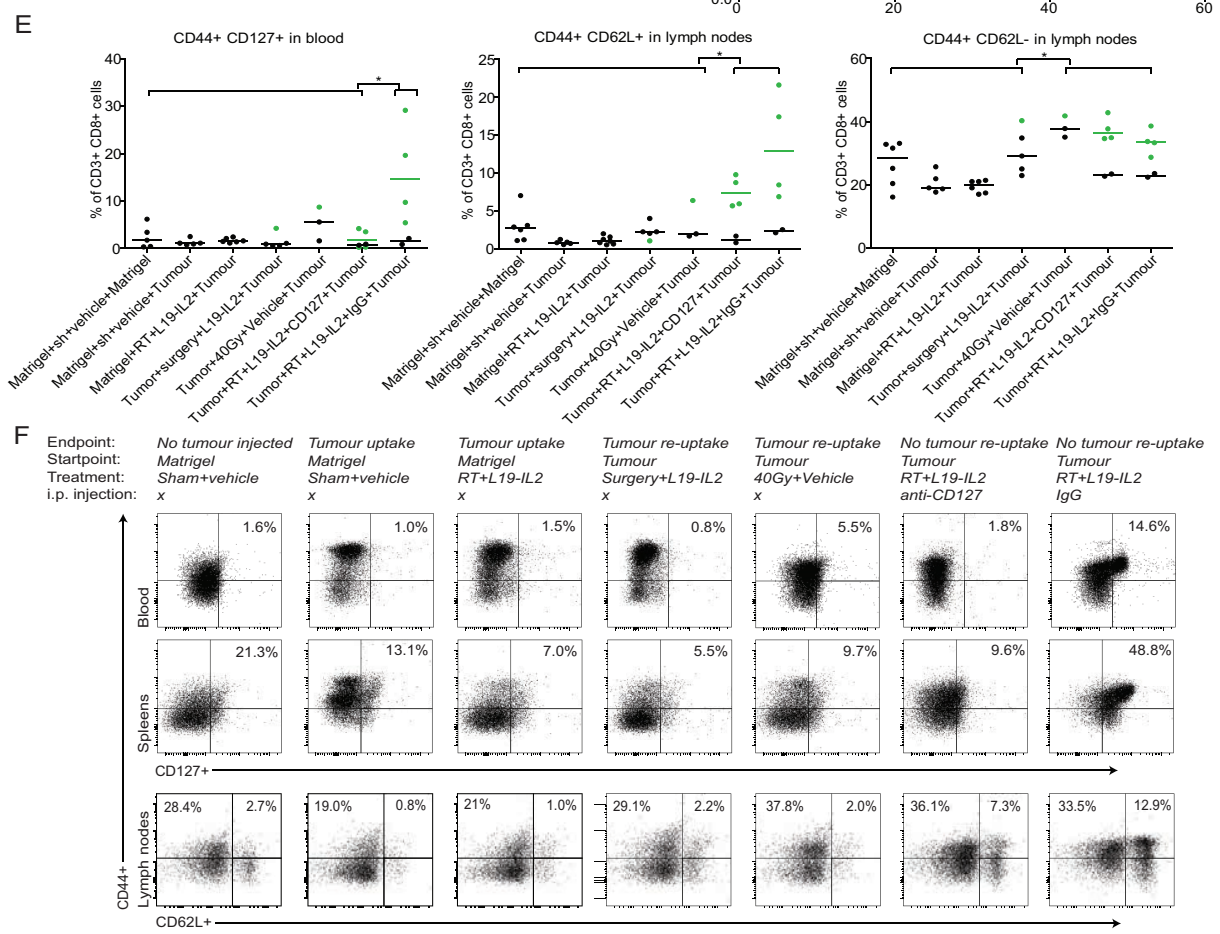

Figure 5: RT + L19-IL2 triggers long-lasting immunological memory. (A) Time to reach $500 \mathrm{~mm}^{3}$ after tumour (re)-injection. (B) \%CD44 ${ }^{+} C D 127^{+}$cells of $C D 8^{+} T$ cells in spleens. (C) Confirmation of CD127 depletion by FACS. (D) Time to reach $500 \mathrm{~mm}^{3}$ after tumour (re)-injection. (E) \% of $\mathrm{CD} 44^{+} \mathrm{CD} 127^{+}, \mathrm{CD}_{4} 4^{+} \mathrm{CD} 62 \mathrm{~L}^{+}$and $\mathrm{CD} 44^{+} \mathrm{CD} 62 \mathrm{~L}^{-}$ of total $C D 8^{+} T$ cells in the spleens and lymph nodes. (F) Median representation of different immune subsets analyzed by FACS. 
tumour cure by RT + L19-IL2 treatment and on CD127 expressing T cells. For this, 150 days after mice were cured from C51 tumours by RT + L19-IL2, surgery + L19-IL2 or high dose RT (40 Gy) (supplementary Fig. 5), mice were re-challenged with C51 tumours. Two days after CD127 blocking, the expression of $\mathrm{CD} 127$ on $\mathrm{CD}^{+}$cells was significantly lower (1054 [761-1491], p=0.004) as compared with the IgG treated mice (2292 [21692555) (Fig. 5c). Eight out of 12 mice cured by RT + L19-IL2, were not able to form new tumours and blocking of CD127 had no influence on this protective effect (Fig. $5 \mathrm{~d}$ ). In contrast, surgery + L19-IL2 and high dose RT induced significantly less protective effect as compared with the RT + L19-IL2 $(p<0.05)$. Furthermore, non-tumour bearing mice pre-treated with RT + L19-IL2 were all able to form tumours (500mm3) within 13 days, similar to control mice (matrigel + sham RT + vehicle), proving that treatment alone is not sufficient to induce memory effect without prior tumour cure (Fig. $5 \mathrm{~d}$ ).

Endpoint immunological analyses (supplementary Fig. 5) revealed that peripheral (blood) $\mathrm{CD}^{+} \mathrm{T}$ cells have high expression of $\mathrm{CD} 44^{+} \mathrm{CD} 127^{+}$when mice were able to reject tumours (14.6\% [5.4-29.1], $\mathrm{p}<0.05)$ after RT + L19-IL2 as compared with other treatment groups (1.6\% [0.6-4.2] of other groups) (Fig. 5e). These high co-expression levels were not found on the $\mathrm{CD}^{+} \mathrm{T}$ cells of the two mice with tumour growth after rechallenge (2.1 and $0.8 \%$ ), showing that the co-expression of both receptors on $\mathrm{CD}^{+} \mathrm{T}$ cells can distinguish long-lasting immunological protection against tumours (supplementary Fig. 5e). RT + L19-IL2 cured mice able to reject tumours also showed a significantly higher percentage of $\mathrm{CD}_{4} 4^{+} \mathrm{CD} 127^{+}$on $\mathrm{CD}^{+} \mathrm{T}$ cells in spleens (48.8\% [35.468.6, $p<0.05)$ as compared with other treatment groups (8.4\% [4.6-38.5] of other groups). Again, this high co-expression level was not found on the $\mathrm{CD}^{+} \mathrm{T}$ cells of the two mice with tumour growth after re-challenge (4.5 and 5.9\%) (supplementary Fig. 6). Mice cured from C51 tumours by L19 + IL2 receiving IgG instead of CD127 blockage had a significantly higher expression of $\mathrm{CD}_{4} 4^{+} \mathrm{CD} 127^{+}$on $\mathrm{CD} 8^{+} \mathrm{T}$ in lymph nodes $(36.7 \%$ [26.9-52.5), $p<0.05)$ as compared with all other groups except for the high dose RT upon tumour cell rejection (supplementary Fig. 6). These results show that the $\mathrm{CD}_{4} 4^{+} \mathrm{CD} 127^{+}$expression on $\mathrm{CD}^{+} \mathrm{T}$ cells associates with but not causes the immunological protective effect since $\mathrm{CD} 127^{+}$blockage did not show any effect on tumour take after re-challenge. 
CD8+ T cells in lymph nodes of cured mice able to reject tumours have a significantly higher $(p=0.01)$ expression of $\mathrm{CD}_{4} 4^{+} \mathrm{CD}_{2} \mathrm{~L}^{+}$(7.3\% [5.7-9.8] IgG group and 12.9\% [6.921.6] anti-CD127 group) as compared with all other treatment groups (2.0\% [1.0-4.0] of all other groups). Additionally, lymph nodes contained a higher percentage of CD44 ${ }^{+}$CD62L' T cells (36.3\%, [34.5-42.8] IgG group and 33.5\% [28.7-38.6]) CD127 group) as compared with all other treatment groups (28.4\% [17.7-40.3] (Fig. 5e, f). It appears that percentage of splenic $\mathrm{CD} 44^{+} \mathrm{CD} 62 \mathrm{~L}^{+}$and $\mathrm{CD} 44^{+} \mathrm{CD} 62 \mathrm{~L}^{-} \mathrm{CD} 8^{+} \mathrm{T}$ cells were higher in cured mice able to reject tumours as compared with mice with tumour take (supplementary Fig. 6). No significant differences between $\mathrm{CD}_{4} 4^{+} \mathrm{CD} 62 \mathrm{~L}^{+}$expressing $\mathrm{CD}^{+} \mathrm{T}$ cells in blood could be observed between treatment groups (data not shown).

\section{DISCUSSION}

Over the last years, the improved understanding of RT-induced effects on the tumour microenvironment has resulted in the recognition that RT has a novel role as an inducer of the immunogenic death of tumour cells(38). Since this form of cell death is capable of converting the patient's tumour into an in situ vaccine, it can initiate an anti-tumour immune response (39), which may be further increased when combined with immunotherapeutic approaches (38). The selective delivery of IL2 to tumour vascular components via the L19-IL2 fusion protein is a novel promising immunotherapy approach $(22,36,40)$ and can enhance the therapeutic potential of RT in ED-B positive tumours in a CD8 ${ }^{+}$T cell (30) or NK cell mediated manner (41). These highly synergistic preclinical findings resulted in the initiation of a phase I clinical study in our institute (NCT02086721), which is currently ongoing. Since the therapeutic effect of this combination treatment is immune mediated, we hypothesized that it can elicit Out-ofField Radiolmmune (OFRI) effects targeting macroscopic tumours directly and preventing new tumour formation (recurrence) later on.

In this study, we have investigated three different RT schedules in combination with L19-IL2 including single dose and fractionated irradiation regimens. In all schedules, irradiated tumours were cured when radiation was combined with L19-IL2. Furthermore, we have shown for the first time that the single RT dose + L19-IL2 mediated OFRI effect retarded tumour growth significantly and even cured $20 \%$ of the non-irradiated tumours. Since it is known that the RT dose, schedule and technique may provoke diverse systemic immune responses (5), we additionally used two fractionated RT regimes ( $5 \times 2$ Gy and $5 \times 5 G y$ ) in combination with L19-IL2. Indeed, several preclinical studies showed significant growth delay and complete remission outside the RT field when immunotherapy (anti-CTLA-4) was combined with fractionated RT but not with a large single dose $(42,43)$. In the present study, we have shown that a significant growth delay of tumours outside the RT field could be observed using fractionated combination 
treatment schedules, however, none of these tumours could be cured, demonstrating that a single high radiation dose is required to trigger RT + L19-IL2 mediated curative OFRI effects. In agreement with our results, a single RT dose (20Gy) initiated immune responses and tumour (including non-irradiated metastases) growth delay when combined with a $\mathrm{CD}^{+} \mathrm{T}$ cell activating immunotherapy (44). This systemic anti-tumour effect was not observed when combined with fractionated RT (4 × 5Gy) (45). Differences in tumour immunogenicity, composition of immune infiltrating cells at start of (RT) treatment (46), RT doses and different immunotherapeutic approaches may all explain the contradictory results to induce anti-tumour effects outside the RT field between different research groups. Fractionated RT might induce a more immunosuppressive microenvironment, favoring the use of checkpoint inhibitors instead of immuneactivating therapies such as L19-IL2, to obtain an optimal OFRI response.

Mice treated with 15Gy + L19-IL2 revealed on day 4 after start treatment an increase in $\mathrm{CD}^{+}$but not in $\mathrm{CD}^{+} \mathrm{T}$ cell infiltration in tumours outside the RT field, indicating an association of the $\mathrm{CD}^{+}{ }^{+} \mathrm{T}$ cells with the observed long-lasting OFRI effect. Despite we did not observe an increased percentage of $\mathrm{CD}^{+} \mathrm{T}$ cells inside the non-irradiated tumours at this time point, depletion of $\mathrm{CD}^{+} \mathrm{T}$ cells abrogated the OFRI effect of the combination therapy significantly. The known role of $\mathrm{CD}^{+} \mathrm{T}$ cells, to recognize tumourassociated antigens and to bring $\mathrm{CD}^{+} \mathrm{T}$ cell responses up to a measurable level (47), may be the explanation why we did observe a significant increase in infiltrating $\mathrm{CD} 4^{+} \mathrm{T}$ cells but not yet $\mathrm{CD} 8^{+} \mathrm{T}$ cells in non-irradiated tumours; it might be a matter of timing. Furthermore, we have shown that depletion of $\mathrm{CD}^{+}{ }^{+}$or $\mathrm{CD}^{+} \mathrm{T}$ cells at day 5 has no or little effect on tumour cure of the primary tumour. This suggests that the anti-tumour immune response against the irradiated tumour was complete at start of depletion, enabling us to follow up growth delay effects of the non-irradiated secondary tumours. These data provide direct evidence that the growth delay and complete remission of $20 \%$ of the non-irradiated tumours is predominantly attributed to $\mathrm{CD}^{+} \mathrm{T}$ cells and at least in part to $\mathrm{CD}^{+} \mathrm{T}$ cells. However, in patients the OFRI effects against metastases might be difficult to monitor or predict by 'Immunoscore', which classifies cancer based on tumour immune infiltrate and has prognostic value proven for several malignancies (48) since biopsies of metastatic tumours are often not available. The systemic monitoring of OFRI effects using peptide- $\mathrm{MHCl}$ multimers labeled with DNA barcodes (49) and use of mathematical models (50-52) are interesting alternative options.

Using the ED-B positive CT26 tumour (36) as a secondary tumour, we observed a similar growth delay of the mismatched tumour when administrating 15Gy to the primary C51 tumour in combination with L19-IL2 compared to a C51 secondary tumour, however without showing tumour cure. The common expression of antigen(s) between the irradiated tumour and other tumour sites may be crucial for an anti-tumour immune response outside the RT field (52). Therefore, it is expected that the irradiated C51 tumour and the non-irradiated CT26 tumour indeed share common tumour specific 
antigens and differences in the antigen-expressing repertoire may explain the reduced OFRI effect observed in this mismatched tumour as compared with the matched (C51) tumour. In addition, differences in tumour-derived chemokines, which are responsible for shaping the tumour microenvironment and thereby determining the composition of tumour-infiltrating immune cells (53), differences in hypoxia creating an immunosuppressive tumour microenvironment, or differences in perfusion enabling penetration and binding of L19-IL2, may also explain the difference in vulnerability of the non-irradiated mismatched tumours to the systemic C51 tumour-derived OFRI effect, which warrants further investigation.

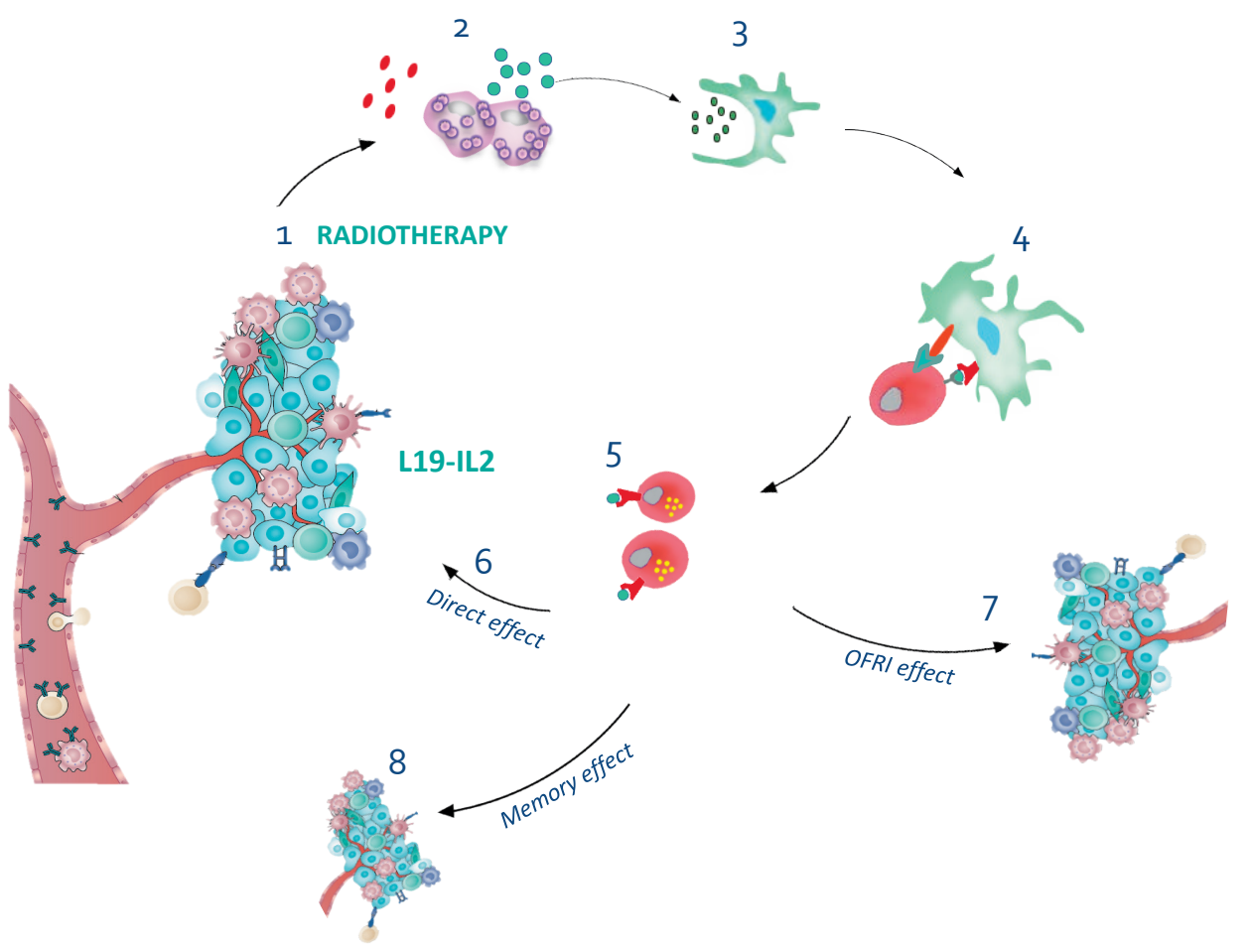

Figure 6: Overview of radiotherapy and L19-IL2 induced anti-tumour effects described in this article. 1. Radiotherapy induces immunogenic cell death, thereby releasing DAMPs (2), creating an in situ vaccine. 3. Tumour-associated antigens are picked up by DCs, that migrate to LNs to activate CD8 ${ }^{+}$T cells (4). L19-IL2 can stimulate the proliferation of tumour specific CD8+ T cells (5) that can now target the irradiated tumours (6), non-irradiated tumours (7) and prevent the formation of new tumours months after tumour cure and termination of the treatment (8).

In this study, we have established the importance of $T$ cells in the execution of the specific RT + L19-IL2 mediated OFRI effect (Fig. 6). Additionally we observed that part of these infiltrating $T$ cells have a regulatory $\left(\mathrm{Foxp}^{+} \mathrm{CD} 25^{+}\right)$phenotype or they express PD1 , resulting in the tolerance and exhaustion of these cells (54). This exhausted $T$ cell 
phenotype as well as increased infiltrate of regulatory $T$ cells was not observed inside irradiated primary tumours, suggesting that large single radiation dose can shift the effector/regulatory balance into an effective immune response. Indeed, it is known that radiotherapy has a broad range of immune stimulating effects on the tumour microenvironment, transforming it into an immunogenic hub (5) and ablating the regulatory $T$ cells (55) making it a highly attractive approach to improve immunotherapeutic efficacy, especially for less immunogenic solid tumours. However, using fractionated $(5 \times 2 \mathrm{~Gy})$ radiotherapy, we could, next to an increase of PD-1 expressing $T$ cells inside non-irradiated tumours, additionally show an increase of PD-1 expressing T cells inside irradiated tumours. This suggests that fractionated RT results in a more exhausted T cell phenotype infiltrate than a single RT dose when combined with L19-IL2. The expression of PD-1 on T cells is identified as an important resistant mechanism to systemic radiotherapy triggered anti-tumour immune responses(56-58). Therefore the observed presence of PD-1 expressing T cells in non-irradiated tumours and in tumours receiving fractionated RT, creates a window-of-opportunity to further increase RT + L19-IL2 OFRI effects by the administration of a PD-1 targeting agent. Furthermore, several PD-1 inhibitors, including nivolumab, have recently obtained FDA approval (37) and show very promising anti-tumour activity in several clinical trials (59, 60). Therefore, a trimodal treatment using RT and L19-IL2 combined with nivolumab might be an interesting approach to further increase the OFRI effect for patients with metastases in a relatively short time-span. Furthermore, direct comparison between non-irradiated tumours of 15Gy and $5 \times 5 G y+$ L19-IL2 treated mice revealed that the single RT dose treated mice have a higher percentage of peripheral $\mathrm{CD}^{+} \mathrm{T}$ cells and a higher percentage of $\mathrm{CD}^{+} \mathrm{T}$ cells infiltrating their non-irradiated tumour. Since it is well known that $\mathrm{CD}^{+} \mathrm{T}$ cells have anti-tumour activity on irradiated tumours (30), and since we have shown in the depletion study that T cells are crucial for a RT + L19-IL2 OFRI effect, these differences may explain why single RT dose is a better inducer of a curative OFRI effect.

Moreover, we have shown in this study that RT + L19-IL2 cured mice are able to develop long-lasting immunological memory (Fig. 6). Mice treated with RT + L19-IL2 did not develop this protective immunity when no tumour was present, showing the importance of the tumour to form the basis for this 'in situ' vaccine that can eventually be translated into a direct (irradiated), indirect (non-irradiated) and long-lasting (rechallenged) anti-tumour immune response. In addition we show that RT is the best trigger to initiate the long-lasting anti-tumour immune response, since surgery + L19-IL2 showed a reduced number of mice able to reject tumour cells upon re-injection, 150 days post cure. High dose RT + vehicle induced significantly worse immunological memory effect, in addition to the elevated normal tissue toxicity, demonstrating the importance of L19-IL2 in triggering the immune response. Immunological analysis revealed a significant increase of $\mathrm{CD}_{4} 4^{+} \mathrm{CD} 62 \mathrm{~L}^{+}$and $\mathrm{CD} 44^{+} \mathrm{CD} 62 \mathrm{~L}^{-}$expression on $\mathrm{CD} 8^{+} \mathrm{T}$ 
cells in the lymph nodes. It has been reported that effector cells have a high expression of CD44 and a low expression of CD62L while central memory cells have a high expression of both CD44 and CD62L (61-63). Therefore, we conclude that effector T cells and central memory $T$ cells are significantly increased in the lymph node compartments when mice are able to reject tumours after they are cured. Furthermore, we show that mice able to reject tumours have a significantly high expression of $C D 44^{+} C D 127^{+}$on splenic, lymph nodes and on circulating $\mathrm{CD}^{+} \mathrm{T}$ cells (blood). It has been shown that the expression of CD44 and CD127 (i.e. the receptor for IL7) on $\mathrm{CD}^{+} \mathrm{T}$ cells can classify these cells as effector memory $T$ cells (61-63), and indeed the expression of CD127 is a hallmark of primed $\mathrm{CD}^{+} \mathrm{T}$ cells to develop into long-lived memory cells $(61,64-66)$. The expression of $\mathrm{CD} 44^{+} \mathrm{CD} 127^{+}$on $\mathrm{CD}^{+} \mathrm{T}$ cells seems to be even more pronounced in the blood, making this expression profile of great interest as a potential biomarker for further selection purposes. A selection can be made to distinguish patients with and without long-term anti-tumour potential. It creates the opportunity to adapt treatment modalities for the latter group, in order to obtain optimal long-lasting anti-tumour effects.

To test the hypotheses in the present proof-of-principle study we have used the wellestablished and -characterized C51 tumour model. To mimic metastasizing cancer, we implanted secondary tumours resembling metastasis (or a secondary tumour in mismatched experiments) outside the radiation field. The choice of this experimental model in these proof-of-principle experiments is governed by the possibility to control experimental conditions such as tumour volume at start of treatment and enable precise irradiation of the primary tumour as wells as accurate daily tumour monitoring, Importantly, all secondary tumours were clearly macroscopic and growing, which reduces potential bias resulting from incomplete tumour take due to technical reasons. It remains to be determined whether RT combined with L19-IL2 can induced the OFRI effect in other models of tumour metastases.

To conclude, this study shows for the first time that a single dose, but not fractionated RT can result in a curative OFRI effect when combined with systemic L19-IL2 treatment dependent on T cells. Mismatching the secondary tumours attenuates this effect and might depend on the percentage of shared (neo)antigens. Single dose RT combined with L19-IL2 leads to an elevation of T cell infiltration in the non-irradiated tumours with a more immunosuppressive phenotype, a phenomenon which is enhanced upon fractionated irradiation. Finally, in this study we have shown that RT + L19-IL2 can induce a long-lasting immunological protection against tumours, which is associated with the presence of effector and central memory T cells (https://youtu.be/aOeJXn 7wNVQ). 


\section{MATERIALS AND METHODS}

Tumour cell lines/ reagents/Antibodies for in vivo studies

Exponentially growing C51 and CT26 mouse colon carcinoma cell lines (kindly provided by Philogen S.p.A., Siena, Italy) were cultured in Dulbecco's Modified Eagle Medium (DMEM) and Roswell Park Memorial Institute (RPMI) (Lonza) respectively, supplemented with $10 \%$ fetal calf serum (FCS) in a humidified $5 \% \mathrm{CO}_{2}$ chamber at $37^{\circ} \mathrm{C}$.

The L19-IL2 immunocytokine (Philogen S.p.A.) was diluted with sterile phosphate buffered saline (PBS, Lonza) to concentrations of $200 \mu \mathrm{g} / \mathrm{ml}$. For in vivo depletion experiments, the monoclonal antibodies anti-CD8a (clone YTS 169.4), anti-CD4 (clone YTS 191) and the isotype control anti-KLH rat IgG2b (clone LTF-2) (Bio X Cell, Lebanon, USA) were diluted with sterile PBS to a concentration of $1.67 \mathrm{mg} / \mathrm{mL}$. For in vivo blocking experiments, the monoclonal anti-CD127 (clone A7R34) and rat IgG2a isotype control (clone 2A3) antibodies (Bio X Cell, Lebanon, USA) were diluted with sterile PBS to a concentration of $2 \mathrm{mg} / \mathrm{mL}$.

\section{Mice and in vivo experiments}

All experiments were performed in accordance with local institutional guidelines for animal welfare and were approved by the Animal Ethical Committee of the University of Maastricht. To induce tumours, approximately 8 weeks old immune competent Balb/c (Harlan Laboratories) mice were subcutaneously injected with $1.5 \times 106$ syngeneic C51 tumour cells suspended in Basement Membrane Matrix (MatrigelTM, BD Biosciences) 10 days prior to RT. Tumours were irradiated upon a tumour volume between 210$296 \mathrm{~mm}^{3}$ with a single dose of $15 \mathrm{~Gy}$ on day 0 or $5 \times 2$ Gy (day 0, 1, 2, 3, 4) fractionated

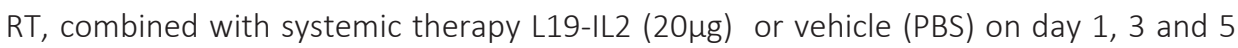
(Fig. 1a, 3a). For the OFRI experiments, C51 tumour cells were injected as described before (day -10) on the left flank and at 8 days prior to RT, C51 cells $(1.5 \times 106)$ or CT26 cells $(2.0 \times 106)$ were suspended in matrigel and injected in the right flank. The volumes or primary and secondary tumours at start of treatment were not significantly different between different treatment groups in all experiments (supplementary table 1).

To be able to compare tumour responses between 15Gy single dose and a fractionated regime we calculated dose per fraction do be delivered once a day for 5 days that results in the same biological effect as single dose RT using the linear quadratic formalism (67). We assumed that the $\alpha / \beta$ ratio of the mouse tumours equals to $10 \mathrm{~Gy}$. Dose recovered per day due to proliferation (Dprolif) was not taken into account in calculations because published data on repopulation of mouse tumours demonstrated that repopulation rate in differentiated adenocarcinomas, mammary carcinoma and in fast fibrosarcoma did not increase in the first week of radiotherapy(68). We calculated 
that $5 \times 5 G y$ (day $0,1,2,3,4)$ should give the same biological effect as a single dose of $15 \mathrm{~Gy}$. This fractionated schedule was delivered only to the left tumour in combination with L19-IL2 (day 1, 3 and 5) (Fig. 4a).

Tumour growth was monitored every 48 hours; tumour dimensions were measured using a Vernier caliper until endpoint, defined as 4 times starting volume (T4XSV). Tumour volume was calculated using the following formula: $(\pi / 6) \times$ length $\times$ width $\times$ height each dimension corrected (minus $0.5 \mathrm{~mm}$ ) for the skin thickness. For all these growth delay experiments, tumour volumes were normalized to day 0 . In parallel, flow cytometry analysis of immunological parameters in tumours and blood at day 4 or day 5 of the treatment schedules was performed.

To evaluate the causal relationship between the presence of $\mathrm{CD}^{+}$or $\mathrm{CD}^{+} \mathrm{T}$ cells and tumour growth delay of the non-irradiated tumours, an experimental set-up was designed to deplete these cells (day 5, 8, 11, Fig. 2a) in C51 tumour-bearing Balb/c mice. Depletion of the cells has been confirmed by flow cytometry analysis (see below) of blood samples collected via puncture of the saphenous vein. Tumour volume data was normalized to start of depletion (day 5).

For the immune memory effect study, animals were challenged with $1.5 \times 10^{6} \mathrm{C} 51$ tumour cells suspended in Matrigel or vehicle on the left flank. When tumours reached an average volume of $200 \mathrm{~mm}^{3}$, different treatment schedules were delivered as depicted in supplementary Fig. 5. Animals that demonstrated long-term survival (tumour cure) were re-challenged 150 days post-implantation with $1.5 \times 10^{6}$ C51 tumour cells suspended in Matrigel (or vehicle) on left and right flanks. Tumour growth was monitored as described above and time to reach $500 \mathrm{~mm}^{3}$ was calculated. Only animals that did not have tumour uptake of left and right tumours were considered to have a long-term immune memory effect. For CD127 in vivo blocking experiments, mice were injected intraperitoneally with anti-CD127 or rat IgG2a isotype control monoclonal antibodies one day before tumour re-challenge and then every 48 hours for 3 weeks. Blockade of CD127 has been confirmed in $\mathrm{CD}^{+}$peripheral blood mononuclear cells in blood samples extracted by saphenous vein puncture after second dose of blocking antibody was delivered (Fig. 5c).

\section{Surgical excision of tumours}

Before surgical excision of tumours, the skin around the tumour was shaved and the area was disinfected with $70 \%$ ethanol. All surgical procedures were done under general anesthesia with isoflurane inhalation. The surgical area was sterilized with iodine solution and a sterilized scalpel was used to make an elliptic incision around the tumour to dissect it away from the flank. To stop the bleeding, an electrocautery was used to close blood vessels and the surgical incision was closed by stitching using the U-stich with an 
absorbable suture. Post-operative care of the animals included the administration of $300 \mathrm{mg} / \mathrm{kg}$ paracetamol to reduce discomfort.

\section{Flow cytometry}

Flow-cytometric analysis was performed on immune cells isolated from spleens, lymph nodes, blood and tumours. Single cell suspensions were obtained using a gentleMACS dissociator and filtered through a $70 \mu \mathrm{m}$-pore cell strainer (Greiner, Bio-one). Additionally, tumours were enzymatically digested with a tumour dissociation kit (Miltenyi Biotec B.V.) before making single cell suspensions. Red blood cells lysis was performed on single cell suspensions of blood, spleens and tumours using RBC lysis buffer (eBioscience, San Diego, CA). Cells were stained with PBS containing 2\% FCS, incubated with FC-block CD16/CD32 (clone 2.4G2; BD Biosciences, San Jose, CA) and stained with a combination of following the antibodies for cell surface markers: antiCD45-V500, PE, FITC, APC and PE-Cy7 (clone 30F-11), anti-CD3-FITC (17A2), anti-CD4APC-H7 (GK1.5), anti-CD8a-V500 (53-6.7), anti-CD19-PE (1D3), anti-CD25-APC (PC61), anti-CD44 APC-Cy7 (IM7), anti-CD127-PE (clone SB/199) and anti-CD127-PE (A7R34) (BD Biosciences); anti-NKp46-APC (29A1.4.9, Miltenyi Biotec B.V., Leiden, The Netherlands); anti-CD45-PerCP (30-F11), anti-CD152 (CTLA-4)-Brilliant Violet 421 (UC10-4B9) and anti279 (PD-1)-PE-Cy7 (RMP1-30) (Biolegend); anti-CD3e- eFLUO 450 (145-2c11), anti-CD4FITC (RM4-5), anti-CD62L-PE-Cy7 (Mel-14) (eBioscience). For intracellular staining, FC blockade and cell surface markers staining was performed, cells were washed with a fixation/permeabilization working solution (eBioscience) according to manufacturer's guidelines and then stained with anti-mouse/rat FOXp3-PE staining set (FJK-16s; eBioscience). Eight-colour flow cytometric analysis was performed with a FACSCanto II instrument (BD Biosciences). Data were analyzed with FACSDiva v6.1.2 (BD Biosciences) and FlowJo v10.0.8 (Tree Star) software. The total $\mathrm{CD} 45^{+}$immune cells were selected from the viable population of cells (filtered for debris and doublets) for further subclassification according to the strategy described previously (30). Staining to assess the expression of immune memory markers were performed on freshly isolated and frozenthawed samples of spleens and lymph nodes and flow cytometric analysis yielded similar results. Gating strategies are described in Supplementary Fig. 4.

\section{Statistical analysis}

Statistical analyses were performed using GraphPad Prism Software (v5.03, San Diego, CA). For all immune parameters and tumour (start) volumes median [max-min] are reported. The non-parametric Mann-Whitney two-tailed test was used to determine the statistical differences between the different treatment groups. The log-rank (MantelCox) test was used to compare the survival curves. When comparing the tumour 
The immunocytokine L19-IL2: a perfect interplay between radiotherapy and long-lasting systemic anti-tumour responses volumes of three treatment groups, a one-way ANOVA, non-parametric (Kruskal Wallis) test was used. P-values smaller than 0.05 were considered statistically significant.

\section{ACKNOWLEDGEMENTS}

Authors acknowledge financial support from ERC advanced grant (ERC-ADG-2015, $\mathrm{n}^{\circ}$ 694812 - Hypoximmuno), the European Program H2020-2015-17 (ImmunoSABR - $n^{\circ}$ 733008) and Kankeronderzoekfonds Limburg from the Health Foundation Limburg. 


\section{REFERENCES}

1 Demaria, S. \& Formenti, S. C. Radiation as an immunological adjuvant: current evidence on dose and fractionation. Front Oncol 2, 153, doi:10.3389/fonc.2012.00153 (2012).

2 Kaur, P. \& Asea, A. Radiation-induced effects and the immune system in cancer. Frontiers in oncology 2 , 191, doi:10.3389/fonc.2012.00191 (2012).

3 Krysko, D. V. et al. Immunogenic cell death and DAMPs in cancer therapy. Nature reviews. Cancer 12 , 860-875, doi:10.1038/nrc3380 (2012).

4 Green, D. R., Ferguson, T., Zitvogel, L. \& Kroemer, G. Immunogenic and tolerogenic cell death. Nat Rev Immunol 9, 353-363, doi:10.1038/nri2545 (2009).

5 Demaria, S., Golden, E. B. \& Formenti, S. C. Role of Local Radiation Therapy in Cancer Immunotherapy. JAMA Oncol 1, 1325-1332, doi:10.1001/jamaoncol.2015.2756 (2015).

6 Demaria, M. et al. An essential role for senescent cells in optimal wound healing through secretion of PDGF-AA. Developmental cell 31, 722-733, doi:10.1016/j.devcel.2014.11.012 (2014).

7 Demaria, S. et al. lonizing radiation inhibition of distant untreated tumors (abscopal effect) is immune mediated. International journal of radiation oncology, biology, physics 58, 862-870, doi:10.1016/j.ijrobp.2003.09.012 (2004).

8 Okuma, K., Yamashita, H., Niibe, Y., Hayakawa, K. \& Nakagawa, K. Abscopal effect of radiation on lung metastases of hepatocellular carcinoma: a case report. J Med Case Rep 5, 111, doi:1752-1947-5-111 [pii] 10.1186/1752-1947-5-111 (2011).

9 Wersall, P. J. et al. Regression of non-irradiated metastases after extracranial stereotactic radiotherapy in metastatic renal cell carcinoma. Acta Oncol 45, 493-497, doi:H628R050154X3538 [pii] 10.1080/02841860600604611 (2006).

10 Mole, R. H. Whole body irradiation; radiobiology or medicine? Br J Radiol 26, 234-241 (1953).

11 Grass, G. D., Krishna, N. \& Kim, S. The immune mechanisms of abscopal effect in radiation therapy. Curr Prob/ Cancer 40, 10-24, doi:10.1016/j.currproblcancer.2015.10.003 (2016).

12 Ehlers, G. \& Fridman, M. Abscopal effect of radiation in papillary adenocarcinoma. Br J Radiol 46, 220222 (1973).

13 Kingsley, D. P. An interesting case of possible abscopal effect in malignant melanoma. Br J Radiol 48, 863866 (1975).

14 Nobler, M. P. The abscopal effect in malignant lymphoma and its relationship to lymphocyte circulation. Radiology 93, 410-412, doi:10.1148/93.2.410 (1969).

15 Ohba, K. et al. Abscopal regression of hepatocellular carcinoma after radiotherapy for bone metastasis. Gut 43, 575-577 (1998).

16 Rees, G. J. Abscopal regression in lymphoma: a mechanism in common with total body irradiation? Clin Radiol 32, 475-480 (1981).

17 Sham, R. L. The abscopal effect and chronic lymphocytic leukemia. Am J Med 98, 307-308, doi:10.1016/S0002-9343(99)80380-5 (1995).

18 Vatner, R. E., Cooper, B. T., Vanpouille-Box, C., Demaria, S. \& Formenti, S. C. Combinations of immunotherapy and radiation in cancer therapy. Frontiers in oncology 4, 325, doi:10.3389/fonc.2014.00325 (2014).

19 Carnemolla, B. et al. Enhancement of the antitumor properties of interleukin-2 by its targeted delivery to the tumor blood vessel extracellular matrix. Blood 99, 1659-1665 (2002).

20 Rosenberg, S. A. IL-2: the first effective immunotherapy for human cancer. Journal of immunology 192, 5451-5458, doi:10.4049/jimmunol.1490019 (2014).

21 Weber, J. S. et al. The use of interleukin-2 and lymphokine-activated killer cells for the treatment of patients with non-Hodgkin's lymphoma. Journal of clinical oncology : official journal of the American Society of Clinical Oncology 10, 33-40 (1992).

22 Johannsen, M. et al. The tumour-targeting human L19-IL2 immunocytokine: preclinical safety studies, phase I clinical trial in patients with solid tumours and expansion into patients with advanced renal cell 
carcinoma. Eur J Cancer 46, 2926-2935, doi:S0959-8049(10)00728-8 [pii] 10.1016/j.ejca.2010.07.033 (2010).

23 Borsi, L. et al. Selective targeting of tumoral vasculature: comparison of different formats of an antibody (L19) to the ED-B domain of fibronectin. International journal of cancer 102, 75-85, doi:10.1002/ijc.10662 (2002).

24 Danielli, R. et al. Armed antibodies for cancer treatment: a promising tool in a changing era. Cancer immunology, immunotherapy : Cll 64, 113-121, doi:10.1007/s00262-014-1621-0 (2015).

25 Eigentler, T. K. et al. A dose-escalation and signal-generating study of the immunocytokine L19-IL2 in combination with dacarbazine for the therapy of patients with metastatic melanoma. Clin Cancer Res 17, 7732-7742, doi:10.1158/1078-0432.CCR-11-1203 (2011).

26 Weide, B. et al. Intralesional treatment of stage III metastatic melanoma patients with L19-IL2 results in sustained clinical and systemic immunologic responses. Cancer Immunol Res 2, 668-678, doi:10.1158/2326-6066.CIR-13-0206 (2014).

27 Danielli, R. et al. Intralesional administration of L19-IL2/L19-TNF in stage III or stage IVM1a melanoma patients: results of a phase II study. Cancer immunology, immunotherapy : Cll 64, 999-1009, doi:10.1007/s00262-015-1704-6 (2015).

28 Robbins, P. F. et al. Mining exomic sequencing data to identify mutated antigens recognized by adoptively transferred tumor-reactive T cells. Nature medicine 19, 747-752, doi:10.1038/nm.3161 (2013).

29 Vogelstein, B. et al. Cancer genome landscapes. Science 339, 1546-1558, doi:10.1126/science.1235122 (2013).

30 Zegers, C. M. et al. Radiotherapy combined with the immunocytokine L19-IL2 provides long-lasting antitumor effects. Clin Cancer Res 21, 1151-1160, doi:10.1158/1078-0432.CCR-14-2676 (2015).

31 Santimaria, M. et al. Immunoscintigraphic detection of the ED-B domain of fibronectin, a marker of angiogenesis, in patients with cancer. Clinical cancer research : an official journal of the American Association for Cancer Research 9, 571-579 (2003).

32 Rossin, R., Berndorff, D., Friebe, M., Dinkelborg, L. M. \& Welch, M. J. Small-animal PET of tumor angiogenesis using a (76)Br-labeled human recombinant antibody fragment to the ED-B domain of fibronectin. Journal of nuclear medicine : official publication, Society of Nuclear Medicine 48, 1172-1179, doi:10.2967/jnumed.107.040477 (2007).

33 Pujuguet, P. et al. Expression of fibronectin ED-A+ and ED-B+ isoforms by human and experimental colorectal cancer. Contribution of cancer cells and tumor-associated myofibroblasts. The American journal of pathology 148, 579-592 (1996).

34 Johnson, E. E. et al. Radiofrequency ablation combined with KS-IL2 immunocytokine (EMD 273066) results in an enhanced antitumor effect against murine colon adenocarcinoma. Clinical cancer research : an official journal of the American Association for Cancer Research 15, 4875-4884, doi:10.1158/10780432.CCR-09-0110 (2009).

35 El-Emir, E. et al. Characterisation and radioimmunotherapy of L19-SIP, an anti-angiogenic antibody against the extra domain B of fibronectin, in colorectal tumour models. Brit J Cancer 96, 1862-1870, doi:10.1038/sj.bjc.6603806 (2007).

36 Schwager, K., Hemmerle, T., Aebischer, D. \& Neri, D. The Immunocytokine L19-IL2 Eradicates Cancer When Used in Combination with CTLA-4 Blockade or with L19-TNF. J Invest Dermatol 133, 751-758, doi:jid2012376 [pii] 10.1038/jid.2012.376 (2013).

37 Sharma, P. \& Allison, J. P. The future of immune checkpoint therapy. Science 348, 56-61, doi:10.1126/science.aaa8172 (2015).

38 Formenti, S. C. \& Demaria, S. Combining Radiotherapy and Cancer Immunotherapy: A Paradigm Shift. J Natl Cancer Inst, doi:djs629 [pii] 10.1093/jnci/djs629 (2013).

39 Formenti, S. C. \& Demaria, S. Systemic effects of local radiotherapy. Lancet Oncol 10, 718-726, doi:S1470-2045(09)70082-8 [pii] 10.1016/S1470-2045(09)70082-8 (2009). 
40 Eigentler, T. K. et al. A dose-escalation and signal-generating study of the immunocytokine L19-IL2 in combination with dacarbazine for the therapy of patients with metastatic melanoma. Clin Cancer Res 17, 7732-7742, doi:1078-0432.CCR-11-1203 [pii] 10.1158/1078-0432.CCR-11-1203 (2011).

41 Rekers, N. H. et al. Combination of radiotherapy with the immunocytokine L19-IL2: Additive effect in a NK cell dependent tumour model. Radiotherapy and oncology : journal of the European Society for Therapeutic Radiology and Oncology, doi:10.1016/j.radonc.2015.06.019 (2015).

42 Demaria, S. et al. Immune-mediated inhibition of metastases after treatment with local radiation and CTLA-4 blockade in a mouse model of breast cancer. Clin Cancer Res 11, 728-734, doi:11/2/728 [pii] (2005).

43 Dewan, M. Z. et al. Fractionated but not single-dose radiotherapy induces an immune-mediated abscopal effect when combined with anti-CTLA-4 antibody. Clin Cancer Res 15, 5379-5388, doi:10780432.CCR-09-0265 [pii] 10.1158/1078-0432.CCR-09-0265 (2009).

$44 \mathrm{Yu}, \mathrm{P}$. et al. Targeting the primary tumor to generate CTL for the effective eradication of spontaneous metastases. Journal of immunology 179, 1960-1968 (2007).

45 Lee, Y. et al. Therapeutic effects of ablative radiation on local tumor require CD8+ T cells: changing strategies for cancer treatment. Blood 114, 589-595, doi:10.1182/blood-2009-02-206870 (2009).

46 Schaue, D., Ratikan, J. A., Iwamoto, K. S. \& McBride, W. H. Maximizing tumor immunity with fractionated radiation. International journal of radiation oncology, biology, physics 83, 1306-1310, doi:10.1016/j.ijrobp.2011.09.049 (2012).

47 Bevan, M. J. Helping the CD8(+) T-cell response. Nat Rev Immunol 4, 595-602, doi:10.1038/nri1413 (2004).

48 Galon, J. et al. Towards the introduction of the 'Immunoscore' in the classification of malignant tumours. The Journal of pathology 232, 199-209, doi:10.1002/path.4287 (2014).

49 Bentzen, A. K. et al. Large-scale detection of antigen-specific T cells using peptide-MHC-I multimers labeled with DNA barcodes. Nature biotechnology 34, 1037-1045, doi:10.1038/nbt.3662 (2016).

50 Serre, R. et al. Mathematical Modeling of Cancer Immunotherapy and Its Synergy with Radiotherapy. Cancer research 76, 4931-4940, doi:10.1158/0008-5472.CAN-15-3567 (2016).

51 Poleszczuk, J. T. et al. Abscopal Benefits of Localized Radiotherapy Depend on Activated T-cell Trafficking and Distribution between Metastatic Lesions. Cancer research 76, 1009-1018, doi:10.1158/00085472.CAN-15-1423 (2016).

52 Demaria, S. \& Formenti, S. C. Can abscopal effects of local radiotherapy be predicted by modeling T cell trafficking? J Immunother Cancer 4, 29, doi:10.1186/s40425-016-0133-1 (2016).

53 Coussens, L. M. \& Werb, Z. Inflammation and cancer. Nature 420, 860-867, doi:10.1038/nature01322 (2002).

54 Picarda, E., Ohaegbulam, K. C. \& Zang, X. Molecular Pathways: Targeting B7-H3 (CD276) for Human Cancer Immunotherapy. Clinical cancer research : an official journal of the American Association for Cancer Research, doi:10.1158/1078-0432.CCR-15-2428 (2016).

55 Bos, P. D., Plitas, G., Rudra, D., Lee, S. Y. \& Rudensky, A. Y. Transient regulatory T cell ablation deters oncogene-driven breast cancer and enhances radiotherapy. The Journal of experimental medicine 210, 2435-2466, doi:10.1084/jem.20130762 (2013).

56 Dovedi, S. J. et al. Acquired resistance to fractionated radiotherapy can be overcome by concurrent PDL1 blockade. Cancer research 74, 5458-5468, doi:10.1158/0008-5472.CAN-14-1258 (2014).

57 Park, S. S. et al. PD-1 Restrains Radiotherapy-Induced Abscopal Effect. Cancer Immunol Res 3, 610-619, doi:10.1158/2326-6066.CIR-14-0138 (2015).

58 Taube, J. M. et al. Colocalization of inflammatory response with B7-h1 expression in human melanocytic lesions supports an adaptive resistance mechanism of immune escape. Science translational medicine 4, 127ra137, doi:10.1126/scitranslmed.3003689 (2012).

59 Borghaei, H. et al. Nivolumab versus Docetaxel in Advanced Nonsquamous Non-Small-Cell Lung Cancer. The New England journal of medicine 373, 1627-1639, doi:10.1056/NEJMoa1507643 (2015).

60 Motzer, R. J. et al. Nivolumab versus Everolimus in Advanced Renal-Cell Carcinoma. The New England journal of medicine 373, 1803-1813, doi:10.1056/NEJMoa1510665 (2015). 
61 Kaech, S. M. et al. Selective expression of the interleukin 7 receptor identifies effector CD8 T cells that give rise to long-lived memory cells. Nature immunology 4, 1191-1198, doi:10.1038/ni1009 (2003).

62 Sallusto, F., Lenig, D., Forster, R., Lipp, M. \& Lanzavecchia, A. Two subsets of memory T lymphocytes with distinct homing potentials and effector functions. Nature 401, 708-712, doi:10.1038/44385 (1999).

63 Wherry, E. J. et al. Lineage relationship and protective immunity of memory CD8 T cell subsets. Nature immunology 4, 225-234, doi:10.1038/ni889 (2003).

64 Huster, K. M. et al. Selective expression of IL-7 receptor on memory T cells identifies early CD4OLdependent generation of distinct CD8+ memory $T$ cell subsets. Proceedings of the National Academy of Sciences of the United States of America 101, 5610-5615, doi:10.1073/pnas.0308054101 (2004).

65 Lang, K. S. et al. Inverse correlation between IL-7 receptor expression and CD8 T cell exhaustion during persistent antigen stimulation. European journal of immunology 35, 738-745, doi:10.1002/eji.200425828 (2005).

66 Madakamutil, L. T. et al. CD8alphaalpha-mediated survival and differentiation of CD8 memory T cell precursors. Science 304, 590-593, doi:10.1126/science.1092316 (2004).

67 Joiner CM, V. d. K. A. Fractionation: the linear-quadratic approach. Basic clinical radiobiology. 4th ed. Boca Raton: CRC Press. pp. 102-119. ( 2009).

68 Trott, K. R. \& Kummermehr, J. Rapid repopulation in radiotherapy: a debate on mechanism. Accelerated repopulation in tumours and normal tissues. Radiotherapy and oncology : journal of the European Society for Therapeutic Radiology and Oncology 22, 159-160 (1991). 


\section{Chapter 5}

Supplementary table 1: Overview of tumour start volumes of different experiments.

\begin{tabular}{|c|c|c|c|}
\hline Figure & Treatment group & $\begin{array}{l}\text { Median [max-min] } \\
\text { tumour volume at start } \\
\text { treatment }\end{array}$ & \\
\hline $1 b, 2 d$ & 15Gy + vehicle secondary tumour & $126 \mathrm{~mm}^{3}[56-146]$ & $P=0.18$ \\
\hline $1 b, 2 d$ & 15Gy + L19-IL2 secondary tumour & $65 \mathrm{~mm}^{3}[38-257]$ & \\
\hline $1 b$ & $5 \times 2 \mathrm{~Gy}+$ vehicle secondary tumour & $67 \mathrm{~mm}^{3}[36-119]$ & $P=0.41$ \\
\hline $1 b$ & $5 \times 2 G y+$ L19-IL2 secondary tumour & $41 \mathrm{~mm}^{3}[29-149]$ & \\
\hline $1 b$ & $5 \times 2 G y+$ L19-IL2 secondary tumour & $41 \mathrm{~mm}^{3}[29-149]$ & $P=0.11$ \\
\hline $1 b$ & 15Gy + L19-IL2 secondary tumour & $65 \mathrm{~mm}^{3}[38-257]$ & \\
\hline $1 b$ & $5 \times 5 G y+$ L19-IL2 secondary tumour & $98 \mathrm{~mm}^{3}[71-163]$ & \\
\hline $2 b$ & 15Gy + L19-IL2 + depletion CD8 secondary tumour & $166 \mathrm{~mm}^{3}[31-304]$ & $P=0.70$ \\
\hline $2 b$ & 15Gy + L19-IL2 + depletion CD4 secondary tumour & $166 \mathrm{~mm}^{3}[29-276]$ & \\
\hline $2 b$ & $15 G y+$ L19-IL2 + IgG secondary tumour & $165 \mathrm{~mm}^{3}[46-230]$ & \\
\hline $2 d$ & 15Gy + vehicle secondary tumour (СT26) & $109 \mathrm{~mm}^{3}[49-132]$ & $P=0.71$ \\
\hline $2 d$ & 15Gy + L19-IL2 secondary tumour (CT26) & $113 \mathrm{~mm}^{3}[39-247]$ & \\
\hline Supplementary $1 b$ & $15 G y+$ vehicle primary tumour & $241.9 \mathrm{~mm}^{3}[156-291]$ & $P=0.26$ \\
\hline Supplementary $1 b$ & 15Gy + L19-IL2 primary tumour & $267 \mathrm{~mm}^{3}[215-337]$ & \\
\hline Supplementary $1 \mathrm{c}$ & $5 \times 2 \mathrm{~Gy}+$ vehicle primary tumour & $300 \mathrm{~mm}^{3}[167-383$ & $P=0.17$ \\
\hline Supplementary $1 \mathrm{c}$ & $5 \times 2 G y+$ L19-IL2 primary tumour & $209 \mathrm{~mm}^{3}[139-273]$ & \\
\hline
\end{tabular}


A

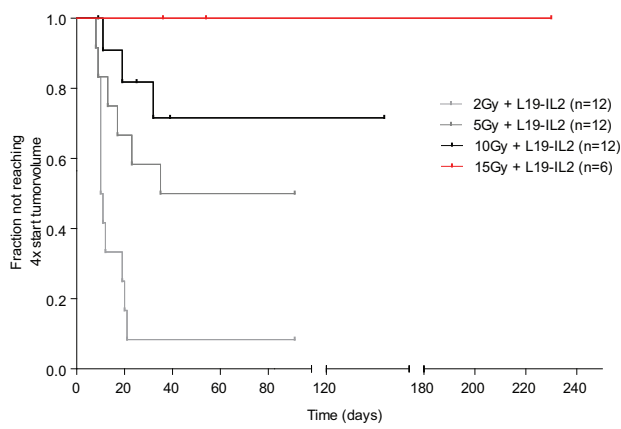

C

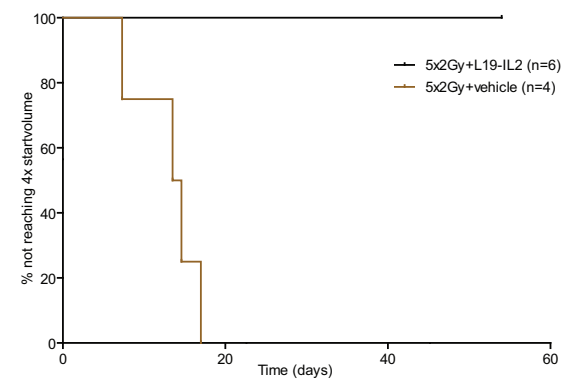

B

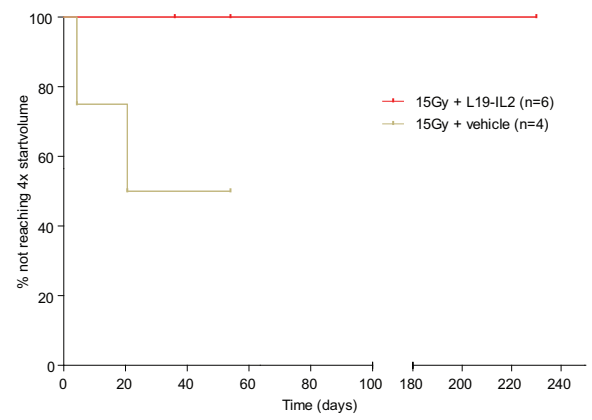

Supplementary figure 1: Growth delay observed in irradiated tumours. (A) Fraction of irradiated tumours not reaching (T4XSV) for the different treatment groups (2, 5 and 10Gy are historical controls (30)). (B) Fraction of irradiated tumours not reaching (T4XSV) treated with 15Gy + L19-IL2 and 15Gy + vehicle. (C) Fraction of irradiated tumours not reaching (T4xSV) treated with $5 \times 2$ Gy + L19-IL2 and $5 \times 2$ Gy + vehicle. 

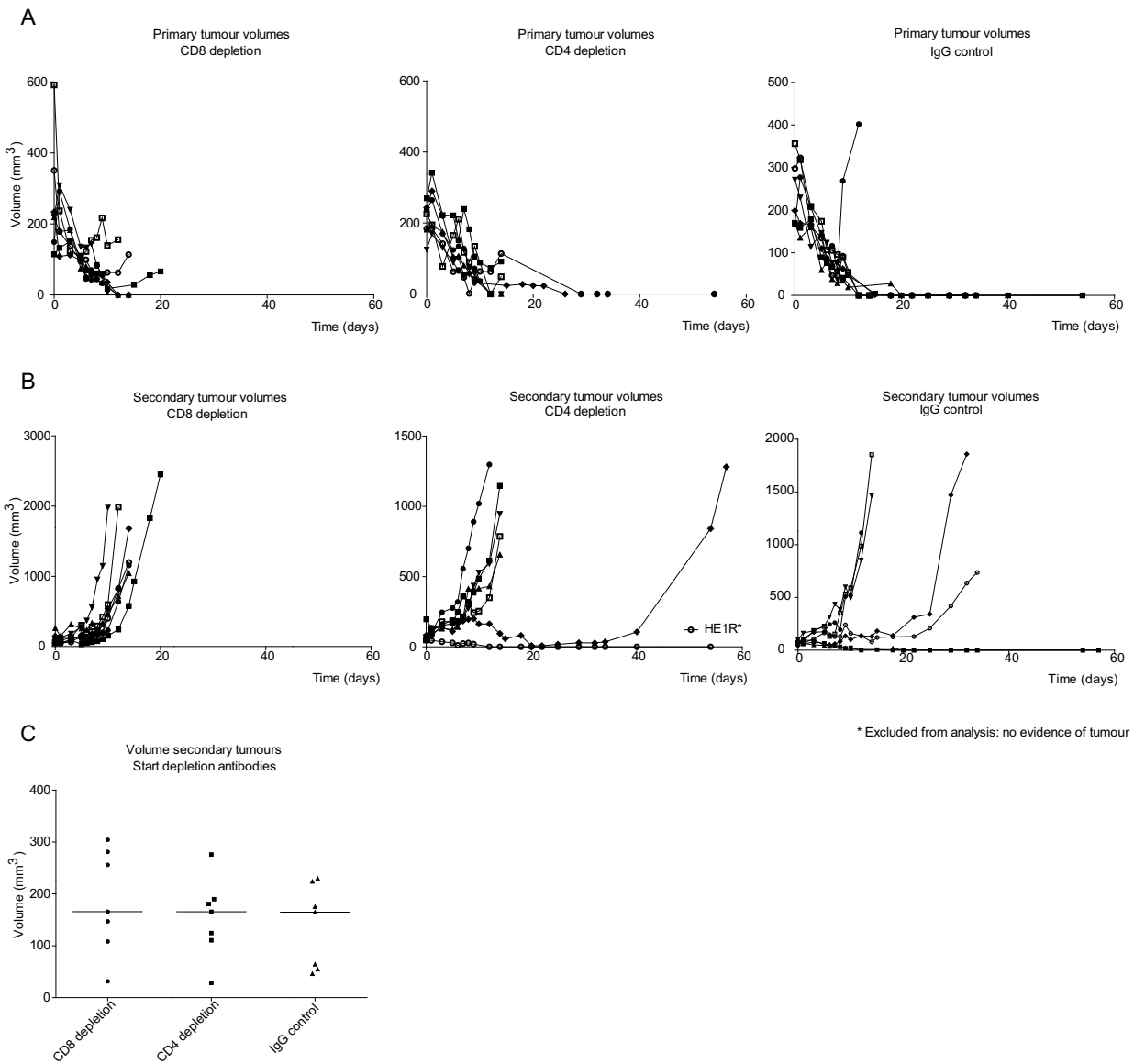

* Excluded from analysis: no evidence of tumour

Supplementary figure 2: Tumour volumes depletion study. (A) Primary tumour volumes of different depletion groups during treatment. (B) Secondary tumour volumes of different depletion groups during treatment. (C) Tumour volumes at start of depletion.

A

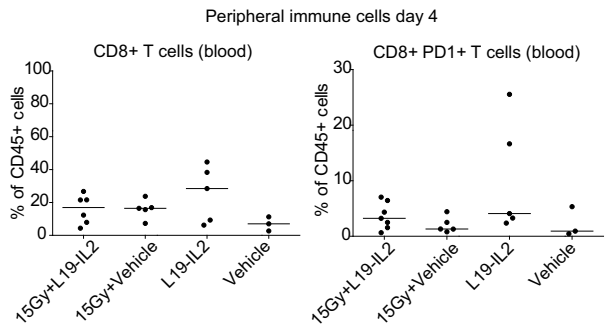

B

Peripheral immune cells day 5
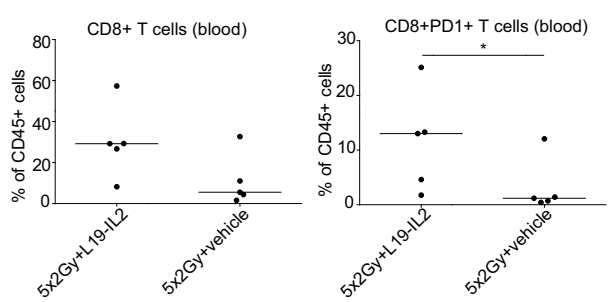

Supplementary figure 3: Peripheral immune cells from different treatment groups. (A) \% of CD8 ${ }^{+}$and CD8 $8^{+} \mathrm{PD}-$ $1^{+}$T cells of the total peripheral $C D 45^{+}$cells among different treatment groups at day 4 . (B) \% of $C D 8^{+}$and $\mathrm{CD}^{+} \mathrm{PD}-1^{+} \mathrm{T}$ cells of the total peripheral $\mathrm{CD} 45^{+}$cells among fractionated treatment groups at day 5 . 


\section{Gating strategy}
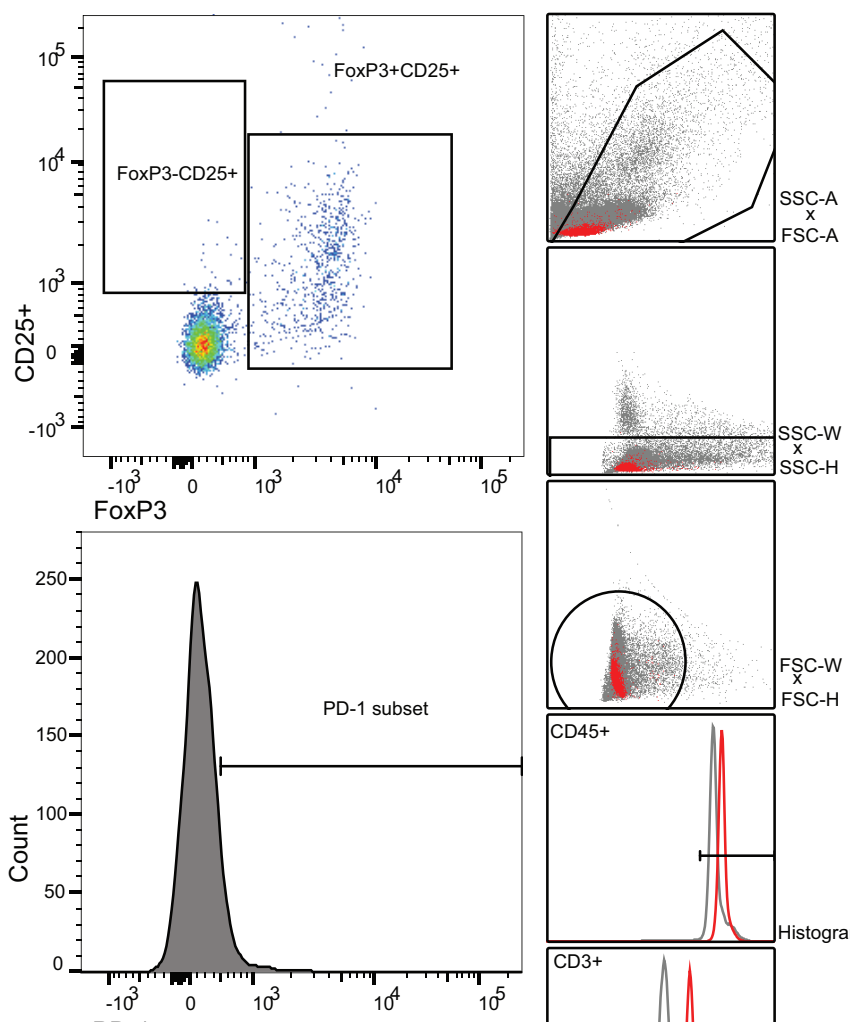

PD-1

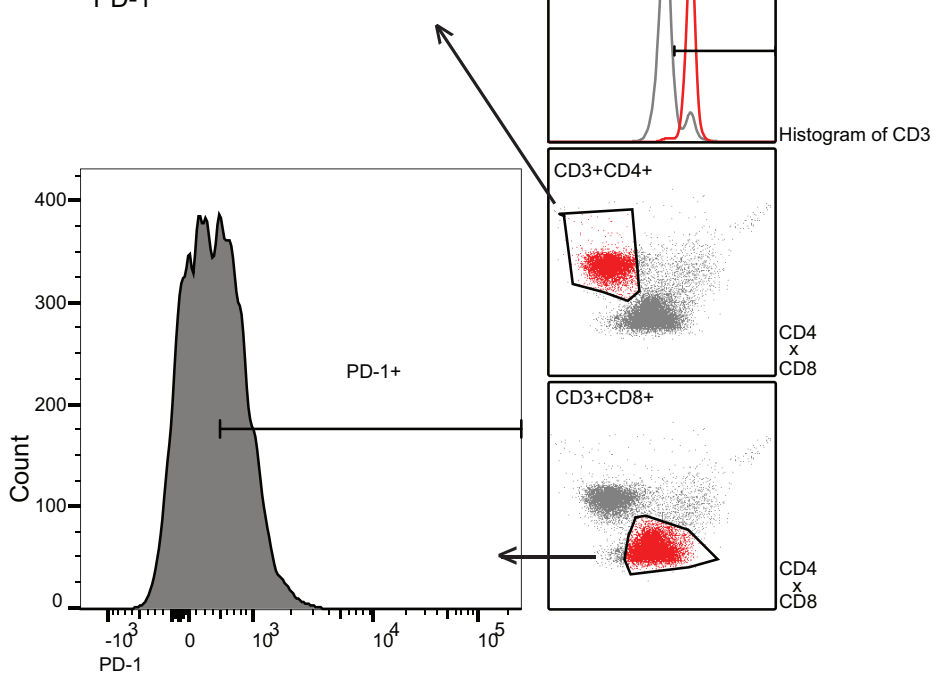

Supplementary figure 4: Gating strategy. Gating strategy used (FACS) to investigate immunosuppressive phenotype T cells. 
A

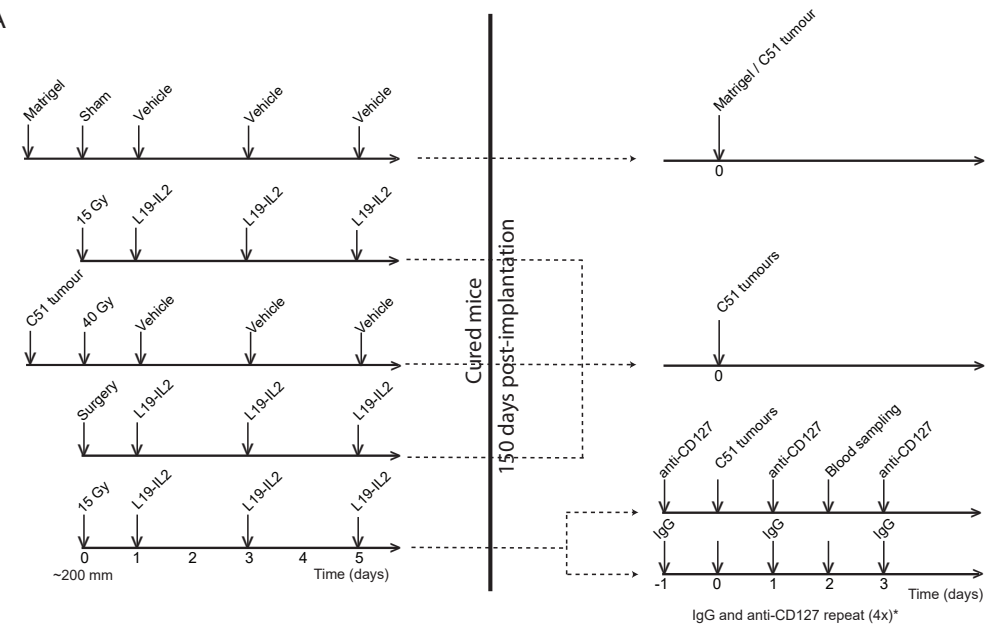

B

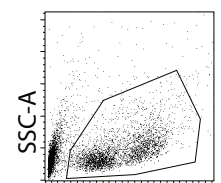

FSC-A
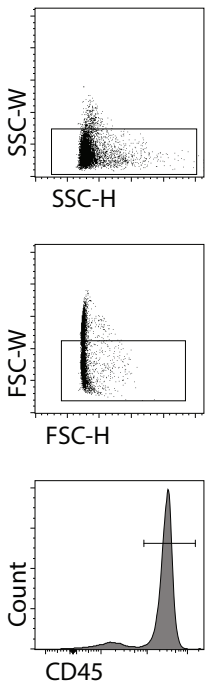
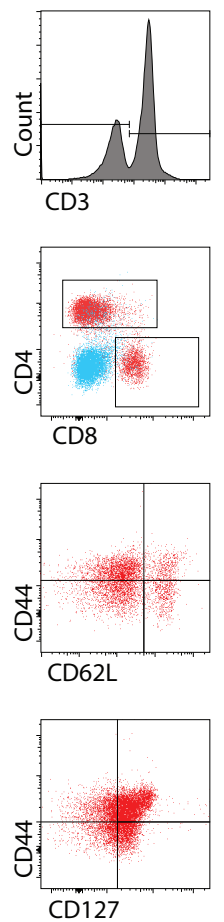

Supplementary figure 5: Study design memory study. (A) Memory study design and (B) gating strategy (FACS). 

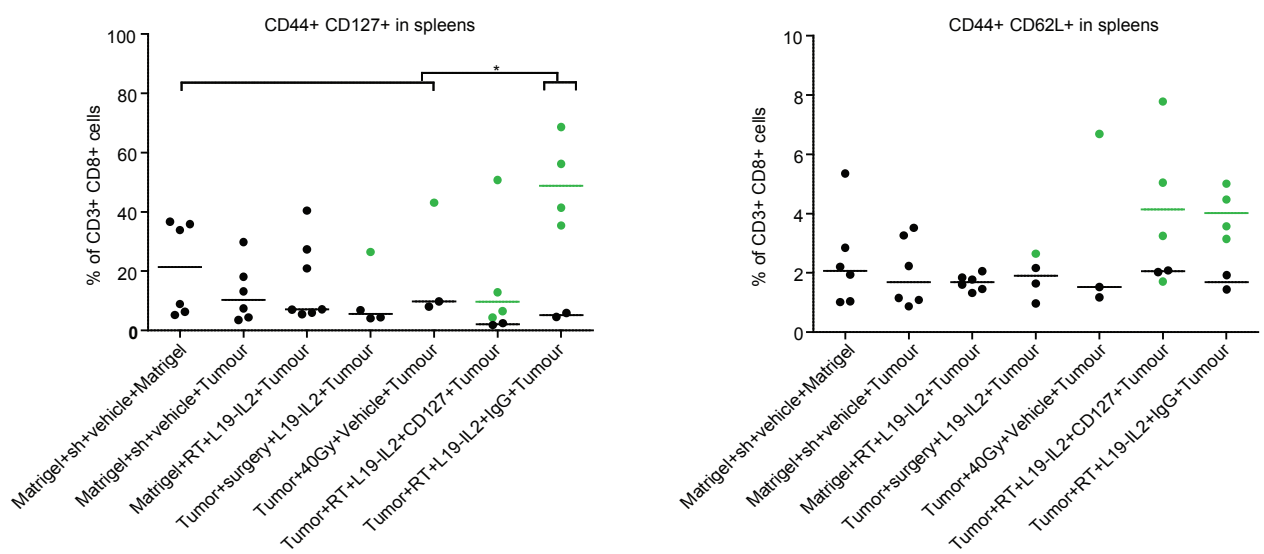

CD44+ CD62L- in spleens
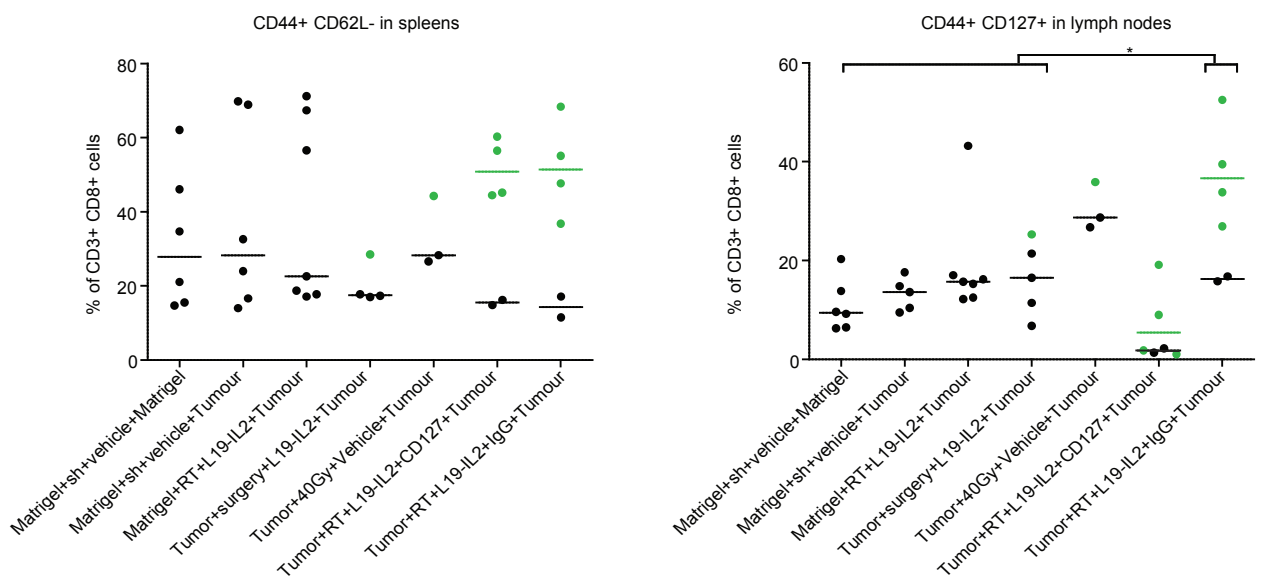

Supplementary figure 6: subsets memory $T$ cells in spleens and lymph nodes.

$\%$ of $\mathrm{CD}_{4} 4^{+} \mathrm{CD} 62 \mathrm{~L}^{+}, \mathrm{CD} 44^{+} \mathrm{CD} 62 \mathrm{~L}^{-}, \mathrm{CD} 44^{+} \mathrm{CD} 127^{+}$of total $\mathrm{CD} 8^{+} \mathrm{T}$ cells in spleens and lymph nodes. 



\section{Chapter}

\section{Combination of radiotherapy with the immunocytokine L19-IL2: additive effect in a NK cell dependent tumour model}

Nicolle. H. Rekers, Catharina. M. L. Zegers, Ala Yaromina, Natasja. G. Lieuwes, Rianne Biemans, Birgit L.M.G. Senden-Gijsbers, Mario Losen, Evert Van Limbergen, Wilfred T.V. Germeraad, Dario Neri, Ludwig Dubois ${ }^{*}$, Philippe Lambin ${ }^{*}$

Published in: Radiother Oncol. 2015; 116(3):438-42 


\section{ABSTRACT}

\section{Background and Purpose}

Recently, we have shown that radiotherapy (RT) combined with the immunocytokine L19-IL2 can induce long-lasting anti-tumour effects, dependent on ED-B expression and infiltration of cytotoxic T cells. On the other hand, in certain tumours, IL2 treatment can trigger a natural killer cell (NK) immune response. The aim of this study is to investigate the therapeutic effect of our combination therapy in the ED-B positive F9 teratocarcinoma model, lacking $\mathrm{MHCl}$ expression and known to be dependent on NK immune responses.

\section{Material and methods}

In syngeneic F9 tumour bearing 129/FvHsd mice tumour growth delay was evaluated after local tumour irradiation (10 Gy) combined with systemic administration of L19-IL2. Immunological responses were investigated using flow cytometry.

\section{Results}

Tumour growth delay of L19-IL2 can be further improved by a single dose of RT administrated before immunotherapy, but not during immunotherapy. Furthermore, treatment of L19-IL2 favours a NK response and lacks cytotoxic T cell tumour infiltrating immune cells, which may be explained by the absence of $\mathrm{MHCl}$ expression.

\section{Conclusion}

An additive effect can be detected when the NK dependent F9 tumour model is treated with radiotherapy and L19-IL2 and therefore this combination could be useful in the absence of tumoural $\mathrm{MHCl}$ expression. 


\section{INTRODUCTION}

Radiotherapy (RT) affects both the tumour and its micro-environment, leading to the release of tumour-associated antigens, damage molecular patterns (DAMPs) and the upregulation of immunomodulatory cell surface molecules, thereby starting an antitumour immune response (1-3). Recently, we have shown that the RT induced antitumour effect can be enhanced by administration of the immunocytokine L19-IL2 (4) which has been shown to have excellent tumour targeting properties and improved therapeutic outcome over IL2 treatment alone (5). The IL2 moiety of this immunocytokine is able to stimulate the proliferation of not only cytotoxic T cells, but also natural killer (NK) cells and can therefore be used to strengthen a broad RT-induced immune response (6). The L19 moiety binds to extra domain-B (ED-B), a part of the fibronectin present in tumour neovasculature and overexpressed in many solid tumours (7). RT combined with L19-IL2 has therefore the potential to create powerful synergistic tumour eliminating effects, as confirmed by our group.

Recently, we reported that the success of this combination treatment is highly dependent on the presence of cytotoxic T cells (4). Cytotoxic $T$ cells are able to recognize and eliminate tumour cells that express tumour-associated peptides on their $\mathrm{MHCl}$. In the high ED-B expressing C51 model, these immune cells were responsible for $75 \%$ of tumour cure and in the intermediate ED-B expressing LLC model an additive effect could be detected. However, in certain tumours, or in later stages of progression, tumour cells can downregulate their $\mathrm{MHCl}$ expression to escape from $\mathrm{T}$ cell mediated killing. As a consequence, tumour cells become more prone to be killed by NK cells, since $\mathrm{MHCl}$ expression of tumour cells inhibits the cytotoxic killing of NK cells (8). As not all tumours demonstrate $\mathrm{MHCl}$ expression, it is unclear if the combination of RT with L19-IL2 is also beneficial in these kinds of tumours. To explore this clinically relevant question, we made use of the ED-B positive F9 teratocarcinoma model as it is independent of T cells and known to induce NK cell responses when treated with IL2based therapeutics $(6,9)$. In this study we investigated whether the combination of RT with L19-IL2 can provoke a NK cell mediated immune response in the F9 tumour model and whether this immune response leads to a therapeutic effect. 


\section{MATERIAL AND METHODS}

\section{In vivo experiments}

All experiments were performed in accordance with local institutional guidelines for animal welfare and were approved by the Animal Ethical Committee of the University of Maastricht. Approximately 8 weeks old 129/SvHsd immune competent mice (Harlan Laboratories) were subcutaneously injected with 3x106 F9 teratocarcinoma cells resuspended in matrigel (MatrigelTM, BD Biosciences). F9 cells were cultured in Dulbecco's Modified Eagle Medium (DMEM; Lonza) supplemented with $10 \%$ fetal calf serum in $0.1 \%$ gelatin coated dishes. Upon an average tumour volume of $200 \mathrm{~mm}^{3}$, therapy was started using three different schedules: (I) a single dose of $10 \mathrm{~Gy}$ radiotherapy (RT) was locally given to tumours on day 0 , combined with systemic

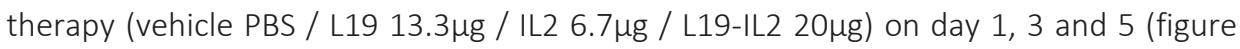
2a), (II) animals were treated as in I, however sacrificed on day 4 of the treatment schedule and used for flow cytometry analysis of tumours, spleen and lymph nodes and (III) systemic therapy was given on day 0, 2 and 4 with local radiotherapy on day 2 (figure 3a). For treatment schedule I and III, tumour growth and treatment toxicity (based on body weight) were monitored on a daily basis until the tumour volume reached 4 times the volume at the start of therapy (T4XSV). To calculate T4xSV volumes were normalized to the start of treatment (day 0 ). To correctly compare control groups with radiotherapy groups in treatment schedules I and III, data were normalized to the start of treatment.

\section{Flow cytometry}

Flow cytometric analysis was performed on single cell suspensions from tumours, spleen and lymph nodes excised at day 4 as previously described (4). Analysis was performed with RBC lysis buffer (eBioscience, San Diego, CA), FC-block CD16/CD32 (BD Biosciences) and a combination of antibodies CD3-FITC, CD4-APC-H7, CD8-PE-CY7, CD19-PE, CD45-V500, CD45-Percp, CD45-PE, CD45-FITC, CD45-APC, CD45-PE-CY7, CD3e-eFLU0450, CD4-FITC, CD8a-V500 (BD Biosciences) and NKp46-APC (Miltenyi Biotec B.V.).

\section{Immunofluorescence}

$7 \mu \mathrm{m}$ frozen sections were fixed in acetone $\left(4^{\circ} \mathrm{C}\right)$ and stained for ED-B, CD31 and CD8 according to previous published methods (4) using L19-SIP and KSF-SIP biotinylated (Philochem), rat anti-CD31 (BD Biosciences) and rabbit anti-CD8 (clone 53.62.7, Department of Pathology, MUMC, Maastricht, The Netherlands), respectively. For $\mathrm{MHCl}$ staining, sections were blocked with $10 \%$ normal goat serum, incubated overnight at 
$4^{\circ} \mathrm{C}$ with primary antibody (rat anti-MHCl, 1:50 in antibody diluent, Abcam). Visualization was done using streptavidin Alexa 488 antibody, goat anti-rabbit IgG Alexa 488 (Life Technologies), goat anti-rat IgG Alexa 488 or goat anti-rat IgG Alexa 594 (BD Bioscience). Fluorescent imaging was performed as described before (4).

\section{Statistics}

Statistical analyses were performed using GraphPad Prism Software (v5.03, San Diego, CA). For all parameters medians with $\left[10^{\text {th }}-90^{\text {th }}\right.$ percentiles $]$ are reported. Nonparametric Mann-Whitney test was used to determine statistical differences. $p$-values smaller than 0.05 were considered statistically significant.

\section{RESULTS}

\section{F9 tumours are ED-B positive and $\mathrm{MHCl}$ negative}

As the immunostimulatory effect of L19-IL2 is dependent on ED-B expression, we verified ED-B expression together with the vascular marker CD31 in the F9-teratoma model. Representative sections are shown in figure 1A. F9 tumours showed high ED-B expression associated with CD31 expression. To investigate a possible mechanism underlying RT+L19-IL2 induced NK cell versus cytotoxic T cell responses, we investigated $\mathrm{MHCl}$ expression of $\mathrm{F9}$ tumours. $\mathrm{F9}$ tumours cells were negative for $\mathrm{MHCl}$ expression, only other structures (i.e. endothelial cells and mouse stromal cells) stained positive for $\mathrm{MHCl}$. The previously investigated tumour model C51, which was dependent on cytotoxic T cells (4) was included as positive control for $\mathrm{MHCl}$ staining (figure 1B). 
A

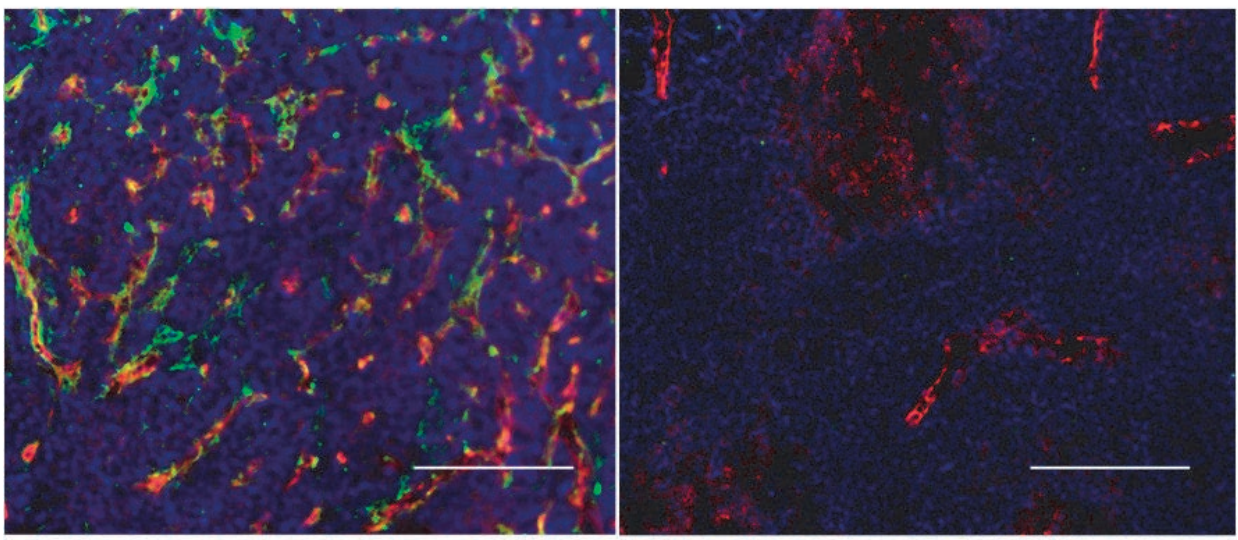

B

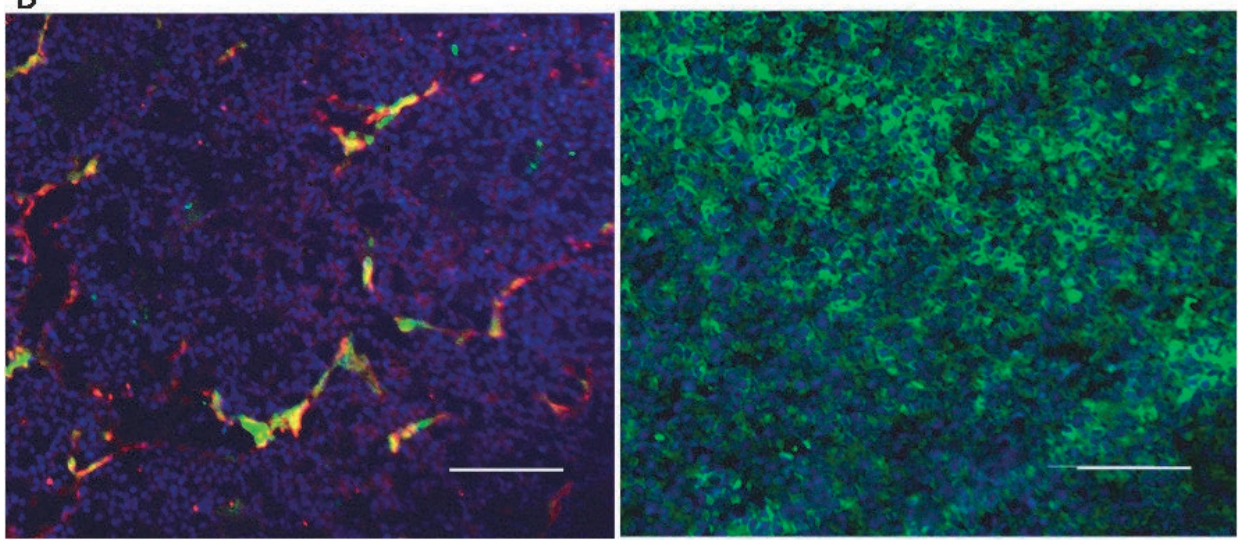

Figure 1A: Representative images of ED-B expression in F9 teratocarcinoma. ED-B expression (left) or control (right) in green, vessels (red) and cell nuclei (blue). B: $\mathrm{MHCl}$ expression (green) and vessels (red) in the F9 teratocarcinoma (left) and $\mathrm{MHCl}$ expression in the C51 colon carcinoma (green), cell nuclei in blue. Scale bar represents $100 \mu \mathrm{m}$. 
Combination therapy provides an additive effect

No toxicities were observed based on body weight measurements and animal welfare monitoring. Combination treatment was performed using two schedules to optimize the best timing for irradiation when compared with L19-IL2 (figure 2A and 3A). According to the animal welfare regulations, we reduced the amount of animals by re-using the tumour growth data of single treatment control groups in schedules I and III.

\section{Radiotherapy before immunotherapy}

Compared to vehicle PBS treated animals (T4xSV = 3.2 [1.7-4.7] days), L19 treatment (3.3 [2.5-4.5] days $\mathrm{P}=0.9$ ) and IL2 (4.4 [2.5-5.6] days $\mathrm{P}=0.2$ ) treatment alone did not lead to an enhanced growth delay. Both RT (4.3 [3.0-8.9] days P<0.05) and L19-IL2 (7.7 [2.713.3] days $\mathrm{P}<0.001)$ monotherapies significantly enhanced tumour growth delay compared to vehicle. Upon combination of RT with L19-IL2, a highly significant antitumour effect (11.5 [7.9-21.4] days $\mathrm{P}<0.0001)$ was observed compared RT+L19 (4.6 [3.0-7.4] days $\mathrm{P}<0.0001$ ) and $\mathrm{RT}+\mathrm{IL} 2$ (4.7 [3.0-6.9] $\mathrm{P}<0.0001)$ (figure 2B). Flow cytometric analysis demonstrated that the percentage of baseline NK cells of all lymphocytes (i.e. $\mathrm{CD} 45^{+}$population) in the tumour was low in vehicle $(0.9 \%$ [0.3-1.2]) and RT+vehicle (0.9\% [0.5-1.9]) treated animals. This number was significantly increased upon treatment with L19-IL2 (5.4\% [2.1-11.5] P<0.001) and RT+L19-IL2 (2.7\% [1.0-11.7] $\mathrm{P}<0.01)$. Furthermore, basal levels of $\mathrm{CD} 8+\mathrm{T}$ cells inside $\mathrm{F9}$ tumours were low and not increased upon treatment (figure 2D). 
A

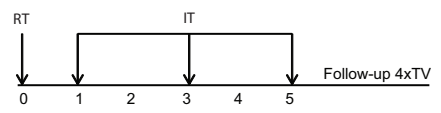

B
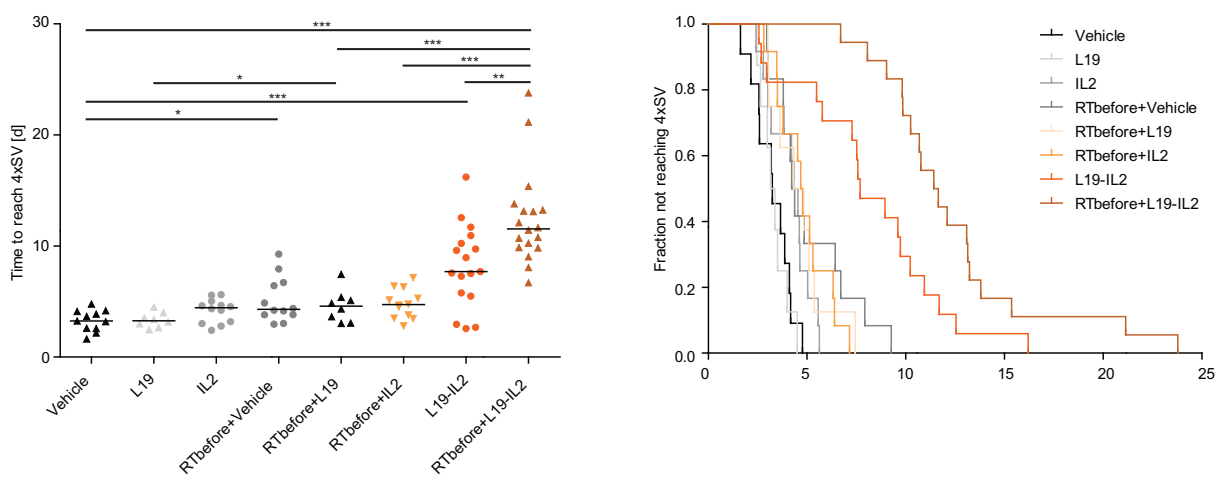

C
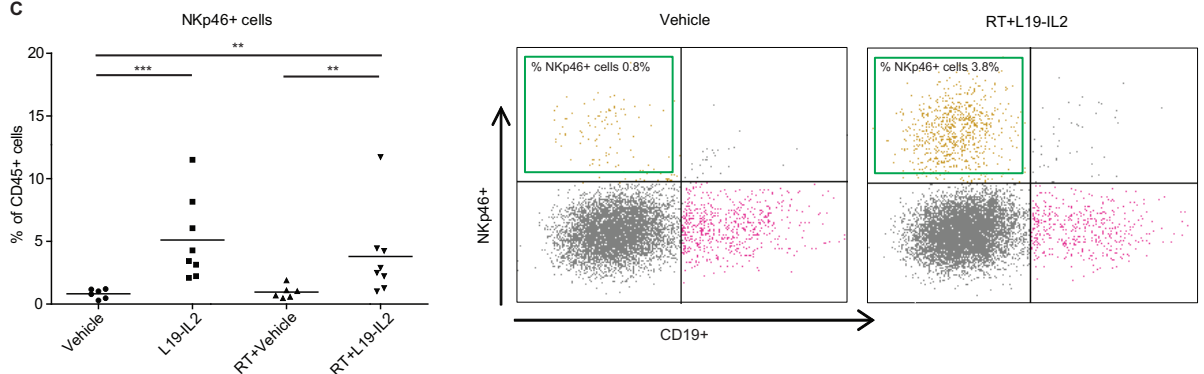

D
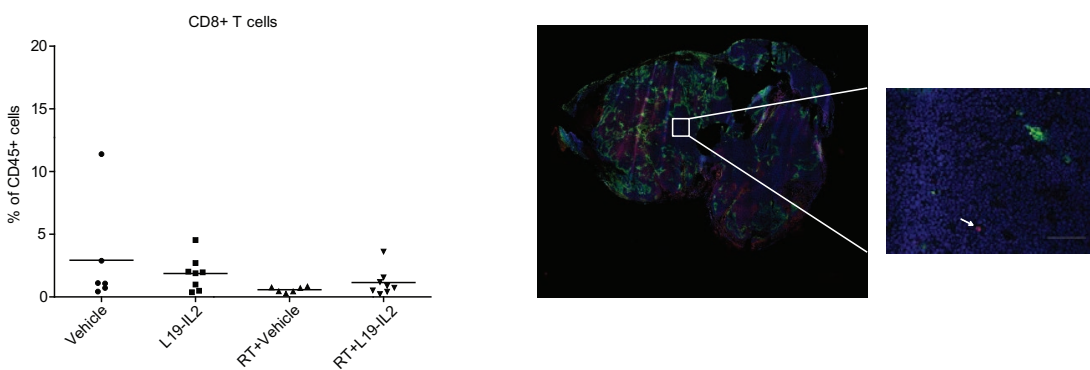

Figure 2A: treatment schedule. B: Time to reach 4 times start volume (T4xSV) for the different treatment groups (left) and Kaplan-Meier curves showing fraction of tumours not reaching T4xSV (right). C,D: Results of flow cytometry analysis, showing the percentage of $N K p 46^{+}$cells $(C)$ and $C D 8+$ cells ( $D$, right) shows of all CD $45^{+}$cells present in the tumour and representative image of a fluorescent CD8 staining. ${ }^{*} P<0.05, * * P<0.01$, $* * * P<0.001$. 


\section{Radiotherapy during immunotherapy schedule}

Local irradiation of F9 tumours with 10Gy (day 2) significantly increased time to reach T4XSV in combination with IL2 (7.1 [5.6-8.9] days $\mathrm{P}<0.001)$ compared to IL2 treatment alone (4.0 [2.7-6.4] days). However, RT did not show any additional effects on L19-IL2 treatment (10.1 [7.6-17.7] days $\mathrm{P}=0.12$ ) compared to the immunocytokine treatment alone (8.9 [3.2-14.9] days). Combining RT with L19-IL2 did significantly delay tumour growth compared to the IL2 combination treatment group $(\mathrm{P}<0.001)$.

A

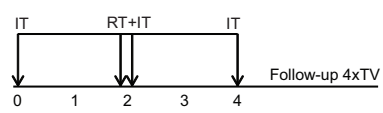

B

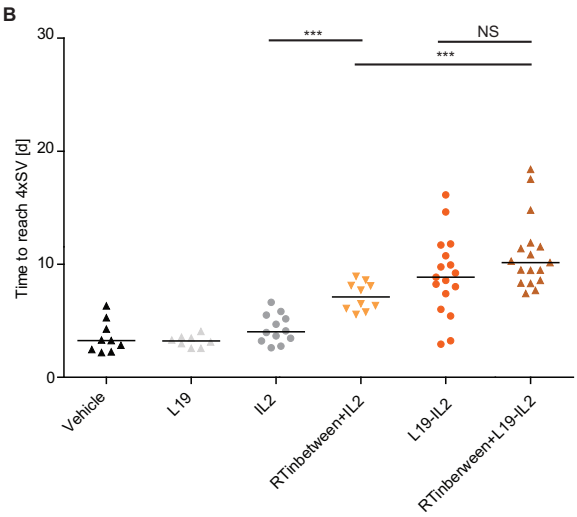

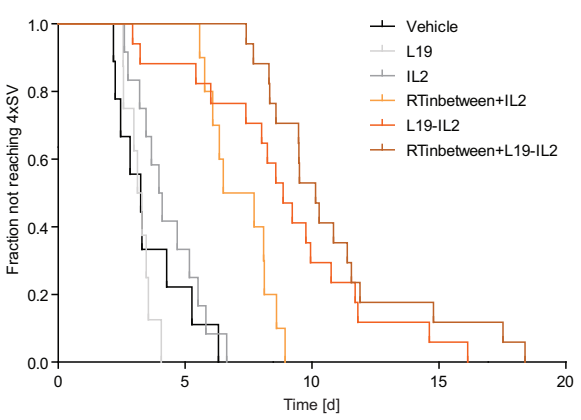

Figure 3: A: Treatment schedule. B: Scatter-plot showing T4xSV (left) and Kaplan-Meier curves showing the fraction of tumours not reaching T4XSV (right). ${ }^{*} \mathrm{P}<0.05$, ${ }^{*} \mathrm{P}<<0.01, * * * \mathrm{P}<0.001$. 


\section{DISCUSSION}

Radiotherapy causes immunogenic death of tumour cells, promotes antigen presentation and alters the tumour microenvironment (10). Recently, we and others have shown that the selective delivery of IL2 to the tumour is a promising approach to enhance the therapeutic potential of $\operatorname{RT}(4,11)$. In this study, we investigated the therapeutic potential and underlying immune response of the RT and L19-IL2 combination treatment in the ED-B positive but $\mathrm{MHCl}$ negative $\mathrm{F} 9$ teratocarinoma tumour model.

Combination of RT with L19-IL2 significantly retarded tumour growth compared to mice treated with RT plus IL2, regardless of RT timing. RT given before L19-IL2 treatment showed a therapeutic gain compared to the RT control groups and compared to single treatment arms. However, RT combined with L19-IL2 did not retarded tumour growth compared with RT plus IL2 treatment group when the single RT dose was delivered during the immunotherapy treatment. Therefore the former effective treatment schedule was used to investigate underlying immunological mechanisms in more detail. Previously, we described that long-lasting anti-tumour effects of RT combined with L19IL2 are highly dependent on expression of ED-B and the presence of cytotoxic T cells inside tumours (4). In this study, we showed that in the ED-B positive F9 teratocarcinoma model the number of infiltrating NK cells, but not the number of cytotoxic T cells, are increased upon L19-IL2 treatment compared to vehicle or RT treatment arms. These results are in agreement with previous publications, where it was shown that, dependent on the tumour model, the efficacy of targeted IL2 treatment by F16-IL2, F8-IL2, L19-IL2 or NHS-IL2 can be based on NK-cells $(9,12)$, T-cells $(11,13-15)$, or a combination of both $(6,16)$. A few possible explanations are available to clarify what favours an NK or T cell response after treatment with RT+L19-IL2. The low expression of $\mathrm{MHCl}$ may be an important one, since down-regulation or inactivation of tumour $\mathrm{MHCl}$ (which is involved in antigen processing and presentation) will prevent cytotoxic T cells from recognizing and eliminating tumours cells (10). On the other hand, downregulation of $\mathrm{MHC}$ expression on their surface causes tumour cells to be more vulnerable for NK cell killing (10), which is in agreement with our recent findings. However, in clinical setting, tumours tend to have a heterogeneous expression of $\mathrm{MHCl}$ (17) and therefore a mixture of cytotoxic T cells and NK cells may become activated when patients are treated with stereotactic body radiation therapy (SBRT) and L19-IL2 in our clinical study (NCT02086721).

Furthermore, treatment with L19-IL2 alone delays tumour growth significantly, which is in line with previous studies (6), and was further enhanced by a single dose of $10 \mathrm{~Gy}$ applied prior to L19-IL2 treatment schedule (figure 2A) but not by a single dose of RT given during the immunotherapy treatment schedule (figure $3 \mathrm{~A}$ ). A possible explanation is that RT the anti-tumour immune response that can be enhanced by L19-IL2, and therefore RT needs to be administrated before immunotherapy. Furthermore, RT might 
be able to kill infiltrating immune cells and therefore RT administrated during the immunotherapy schedule may not have additional benefit.

However, for the systemic IL2 treatment, we detected a significant anti-tumour immune response when administered before RT (figure $3 \mathrm{~A}$ ). This effect was not detected in the other treatment schedule, were IL2 was administered at day 1, 3 and 5 after RT (figure 2A). Similar to our findings, Milas et al (18) showed that stimulation of the innate and adaptive immune system (by CpG oligodeoxynucleotides) prior to a single dose of local RT, is able to improve the outcome for RT treatment in mouse fibrosarcoma. Interestingly, the 'radiosensitizing effect' of systemic IL2 was not detected when IL2 was administered in the targeted form (L19-IL2) before RT. Since L19-IL2 binds to ED-B and therefore stimulates the immune response only locally and since intratumoural basal levels of NK cells are very low, systemic IL2 treatment might have a higher impact compared to L19-IL2 in sensitizing tumours to irradiation. Taken together, administration of IL2 before but not after radiotherapy is able to delay tumour growth in this model and when L19-IL2 is administrated before radiotherapy, this 'radiosensitizing effect' is not present.

In conclusion, combination treatment of RT with L19-IL2 is an efficient treatment combination in the $\mathrm{MHCl}$ negative but ED-B positive $\mathrm{F} 9$ tumour model and effects of L19-IL2 can be further improved by a single dose of RT before immunotherapy treatment. A single RT dose administrated during immunotherapy treatment seems to be less efficient. Furthermore, the data suggest that treatment of L19-IL2 favours NK and lacks cytotoxic $T$ cell tumour infiltrating immune cells, which may be explained by the absence of $\mathrm{MHCl}$ expression by these $\mathrm{F} 9$ tumours.

\section{ACKNOWLEDGEMENTS}

Authors acknowledge financial support from Philogen S.p.A. (Sovicille, Italy), 7th framework program (METOXIA). The micrographs were taken with a confocal spinning disk microscope financed by The Netherlands Organisation for Scientific Research (NWO), grant number 911-06-003. The micrographs in this paper were taken with a confocal spinning disk microscope financed by The Netherlands Organization for Scientific Research (NWO), grant number 911-06-003. 


\section{REFERENCES}

1. Rekers NH, Troost EG, Zegers CM, Germeraad WT, Dubois LJ, Lambin P. Stereotactic ablative body radiotherapy combined with immunotherapy: present status and future perspectives. Cancer Radiother. 2014;18(5-6):391-5.

2. Demaria S, Formenti SC. Radiotherapy effects on anti-tumor immunity: implications for cancer treatment. Frontiers in oncology. 2013;3:128. PMCID: 3660697.

3. Formenti SC, Demaria S. Combining radiotherapy and cancer immunotherapy: a paradigm shift. J Natl Cancer I. 2013;105(4):256-65. PMCID: 3576324.

4. Zegers CM, Rekers NH, Quaden DH, Lieuwes NG, Yaromina A, Germeraad WT, et al. Radiotherapy Combined with the Immunocytokine L19-IL2 Provides Long-lasting Antitumor Effects. Clinical cancer research : an official journal of the American Association for Cancer Research. 2015;21(5):1151-60.

5. List T, Neri D. Immunocytokines: a review of molecules in clinical development for cancer therapy. Clin Pharmacol. 2013;5:29-45. PMCID: 3753206.

6. Carnemolla B, Borsi L, Balza E, Castellani P, Meazza R, Berndt A, et al. Enhancement of the antitumor properties of interleukin-2 by its targeted delivery to the tumor blood vessel extracellular matrix. Blood. 2002;99(5):1659-65.

7. Menrad A, Menssen HD. ED-B fibronectin as a target for antibody-based cancer treatments. Expert Opin Ther Targets. 2005;9(3):491-500.

8. Medzhitov R, Janeway CA, Jr. Decoding the patterns of self and nonself by the innate immune system. Science. 2002;296(5566):298-300.

9. Lode HN, Xiang R, Dreier T, Varki NM, Gillies SD, Reisfeld RA. Natural killer cell-mediated eradication of neuroblastoma metastases to bone marrow by targeted interleukin-2 therapy. Blood. 1998;91(5):170615.

10. Vatner RE, Cooper BT, Vanpouille-Box C, Demaria S, Formenti SC. Combinations of immunotherapy and radiation in cancer therapy. Frontiers in oncology. 2014;4:325. PMCID: 4246656.

11. van den Heuvel MM, Verheij M, Boshuizen R, Belderbos J, Dingemans AM, De Ruysscher D, et al. NHS-IL2 combined with radiotherapy: preclinical rationale and phase $\mathrm{Ib}$ trial results in metastatic non-small cell lung cancer following first-line chemotherapy. J Transl Med. 2015;13(1):32. PMCID: 4320467.

12. Moschetta M, Pretto F, Berndt A, Galler K, Richter P, Bassi A, et al. Paclitaxel enhances therapeutic efficacy of the F8-IL2 immunocytokine to EDA-fibronectin-positive metastatic human melanoma xenografts. Cancer research. 2012;72(7):1814-24.

13. Becker JC, Pancook JD, Gillies SD, Furukawa K, Reisfeld RA. T cell-mediated eradication of murine metastatic melanoma induced by targeted interleukin 2 therapy. The Journal of experimental medicine. 1996;183(5):2361-6. PMCID: 2192587.

14. Xiang R, Lode HN, Dolman CS, Dreier T, Varki NM, Qian X, et al. Elimination of established murine colon carcinoma metastases by antibody-interleukin 2 fusion protein therapy. Cancer research. 1997;57(21):4948-55.

15. Gutbrodt KL, Casi G, Neri D. Antibody-based delivery of IL2 and cytotoxics eradicates tumors in immunocompetent mice. Molecular cancer therapeutics. 2014;13(7):1772-6.

16. Gutbrodt KL, Schliemann C, Giovannoni L, Frey K, Pabst T, Klapper W, et al. Antibody-based delivery of interleukin-2 to neovasculature has potent activity against acute myeloid leukemia. Science translational medicine. 2013;5(201):201ra118.

17. Igney $\mathrm{FH}$, Krammer $\mathrm{PH}$. Immune escape of tumors: apoptosis resistance and tumor counterattack. Journal of leukocyte biology. 2002;71(6):907-20.

18. Milas L, Mason KA, Ariga H, Hunter N, Neal R, Valdecanas D, et al. CpG oligodeoxynucleotide enhances tumor response to radiation. Cancer research. 2004;64(15):5074-7. 


\section{Chapter}

\section{IL2 based immunotherapies: \\ Towards a personalized and curative anti-tumour response}

Rekers NH, Olivo Pimentel V, Yaromina A, Dubois L, Lambin P.

Published in: Transl Cancer Res 2016;5(Suppl 6):S1292-S1294 
In his commentary, Claus Garbe described our study on the combination of radiotherapy (RT) and the immunocytokine L19-IL2 in the F9 fibrosarcoma model (1) and discussed our work in a checkpoint inhibitor related context. The author is positioning interleukin-2 (IL2) based immunotherapies in the so-called 'age of the checkpoint inhibition' and concluded that the role of IL2 and/or L19-IL2 should preferentially be examined in patients not responding to checkpoint blockade, since these patients are not able to develop specific cytotoxic T cell responses. Therefore IL2 based immunotherapeutic approaches might circumvent this problem by activating the innate immune response against the tumour (2).

The comparison of IL2 based therapies and checkpoint inhibitors made in this commentary is based on the fact that checkpoint inhibitors act on the modulation of an already present adaptive immune response (cytotoxic T cells) and IL2 based therapies are only able to trigger the innate immune system (NK cells). Indeed, we have shown that L19-IL2 (an immunocytokine binding to EDB present in the tumour vasculature and known for its locally operating immune modulating effects) is able to cause a significant growth delay associated with an intratumoural increase of NK cells in a $\mathrm{MHCl}$ negative tumour model (1). These effects were significantly higher as compared with equimolar levels of IL2 and are explained by the larger proportion of IL2 reaching the EDB positive tumour by using L19 as a tumour specific vehicle. Therefore, we agree with the statement that the ability of L19-IL2 to activate members of the innate immune system, in our study NK cells, indeed creates an interesting alternative for checkpoint inhibitors in case the cytotoxic T cell 'baseline' is insufficient.

Furthermore, we have shown that the delivery of a single RT dose prior to the L19-IL2 treatment schedule further enhanced the anti-tumour response of this treatment, while a schedule administrating RT during L19-IL2 treatment was less effective. Since NK cells can become activated by L19-IL2 to target $\mathrm{MHCl}$ negative tumour cells directly and do not necessarily need activation via cross-presentation of antigen presenting cells, this may explain why we have found an additive and not a synergistic effect of the combination treatment in this model (1). However, in addition to this tumour model we found long-lasting synergistic and additive effects of RT administrated prior to L19-IL2 in other tumour models. These models were all $\mathrm{MHCl}$ positive, therefore the RT + L19-IL2 combined treatment approach was highly dependent on the action of cytotoxic $T$ cells instead of NK cells (3). In clinical setting, tumours tend to have a heterogeneous expression of $\mathrm{MHCl}(4)$ and therefore a mixture of cytotoxic T cells and NK cells may become activated when patients are treated with a combination of radiation and L19IL2 based immunotherapy.

For decades, the focus of RT related research was on its direct and local effects, depending on DNA damage and the intrinsic repair capacity of irradiated cells (5). However, RT additionally can cause immunogenic cell death of cancer cells, promoting 
the uptake and cross-presentation of released tumour (neo)antigens by dendritic cells (DC) to T cells in the draining lymph node and converting the irradiated tumour into an in situ personalized tumour vaccine (6). The concept that personalized vaccination is based on the recognition of (neo)antigens generated by tumour specific $T$ cells (7), placed the use of RT in a totally different context (8). However, the commentary (2) addresses the issue that not all patients respond to treatment with checkpoint inhibitors via the release of specific cytotoxic T cells, a problem also observed in our $\mathrm{MHCl}$ positive tumour models when treated with L19-IL2 as single treatment. Based on recent publications $(9,10)$ and our observations we believe this may be caused by the insufficient cross-presentation of specific (neo)antigens to cytotoxic $T$ cells and a decreased expression of (neo)antigens on $\mathrm{MHCl}$ by the tumour. In our opinion, RT can be the solution for both of these problems, and therefore RT can be used to further optimize and personalize an immunotherapeutic approach, including L19-IL2 and checkpoint inhibitor treatments. Furthermore, L19-IL2 may be favorable in case the RT triggered immunogenic cell death is not optimally capable of increasing the tumours immunogenicity, i.e. increasing the $\mathrm{MHCl}$ expressing tumour specific (neo)antigens, providing the immune system an extra cytotoxic tool, the NK cells.

Radiotherapy (RT) is one of the major treatment options for cancer and approximately $52 \%$ of all cancer patients receive RT during their treatment. The possibilities of RT to initiate an anti-tumour response by creating an in situ tumour vaccine and its potential to change a tumours (neo)antigen landscape, has the potential to greatly enhance the personalization and effectiveness of immune modulating agents. Indeed, the mechanisms of checkpoint inhibitors relay on the re-activation of already present but exhausted tumour (neo)antigen specific T cells, L19-IL2 function relies more on the activation and proliferation stage of these tumour specific $\mathrm{T}$ cells. In other words, checkpoint inhibitors are able to get rid of the brake, and L19-IL2 is able to push the gas. In our opinion, the first line treatment must consist of RT to create a personalized in situ vaccine and increase a tumours immunogenicity, followed by L19-IL2 to stimulate the proliferation of tumour (neo)antigen specific $T$ cells at the tumour site. When these specific $T$ cells become exhausted, expressing CTLA-4 and/or PD1 immune downregulating molecules, we see a clear role for checkpoint inhibitors. In case RT was insufficient to initiate a proper anti-tumour immune response against the tumour, indeed, L19-IL2 is even able to stimulate NK induced cytotoxicity. However, we believe that a long-lasting (memory effects) and off target (abscopal effects) anti-tumour immune effect can be reached when the right RT dose/schedule will be combined with the right immunotherapeutic approach in order to stimulate an immune response of adaptive origin. 


\section{REFERENCES}

1. Rekers NH, Zegers CM, Yaromina A, Lieuwes NG, Biemans R, Senden-Gijsbers BL, et al. Combination of radiotherapy with the immunocytokine L19-IL2: Additive effect in a NK cell dependent tumour model. Radiotherapy and oncology : journal of the European Society for Therapeutic Radiology and Oncology. 2015.

2. Garbe C. Interleukin-2 as anticancer immunotherapy in the age of checkpoint inhibition. Translational cancer research. 2016;5, Supplement 2.

3. Zegers CM, Rekers NH, Quaden DH, Lieuwes NG, Yaromina A, Germeraad WT, et al. Radiotherapy combined with the immunocytokine L19-IL2 provides long-lasting antitumor effects. Clinical cancer research : an official journal of the American Association for Cancer Research. 2015;21(5):1151-60.

4. Igney $\mathrm{FH}, \mathrm{Krammer} \mathrm{PH}$. Immune escape of tumors: apoptosis resistance and tumor counterattack. Journal of leukocyte biology. 2002;71(6):907-20.

5. Prise KM, Schettino G, Folkard M, Held KD. New insights on cell death from radiation exposure. The Lancet Oncology. 2005;6(7):520-8.

6. Rekers NH, Troost EG, Zegers CM, Germeraad WT, Dubois LJ, Lambin P. Stereotactic ablative body radiotherapy combined with immunotherapy: present status and future perspectives. Cancer Radiother. 2014;18(5-6):391-5.

7. Schumacher TN, Schreiber RD. Neoantigens in cancer immunotherapy. Science. 2015;348(6230):69-74.

8. Formenti SC, Demaria S. Radiation therapy to convert the tumor into an in situ vaccine. Int J Radiat Oncol Biol Phys. 2012;84(4):879-80.

9. Demaria S, Golden EB, Formenti SC. Role of Local Radiation Therapy in Cancer Immunotherapy. JAMA Oncol. 2015;1(9):1325-32.

10. Verdegaal EM, de Miranda NF, Visser M, Harryvan T, van Buuren MM, Andersen RS, et al. Neoantigen landscape dynamics during human melanoma-T cell interactions. Nature. 2016;536(7614):91-5. 
Chapter

\section{Radiotherapy, L19-IL2 and ipilimumab trimodal treatment: a suppressive role of PD-L1?}

Rekers NH, Ala Yaromina, Veronica Olivo Pimentel, Ludwig Dubois, Philippe Lambin In preparation for Oncolmmunology 


\section{ABSTRACT}

Recently, we have shown that the combination of radiotherapy (RT) with L19-IL2 results in RT dose-dependent long-lasting curative anti-tumour effects in an ED-B positive C51 colon carcinoma model. In this study we investigated the possibility of increasing the anti-tumour immune response observed in the lowest RT dose (2Gy) combinational treatment approach by the addition of an anti-CTLA-4 checkpoint inhibitor. We observed that the trimodal treatment (RT + L19-IL2 + anti-CTLA-4) results in an improved anti-tumour response as compared with all other treatments, except for the bimodal treatment (RT + L19-IL2). Strikingly, the addition of anti-CTLA-4 significantly $(p=0.04)$ decreases the anti-tumour response of the RT + L19-IL2 combination treatment and our preliminary data suggest that the PD-L1 immunosuppressive mechanism might explain this difference. 


\section{INTRODUCTION}

Besides its direct cytotoxic effect, tumour irradiation can additionally induce an immunogenic cell death, thereby converting the irradiated tumour into an immunogenic hub and initiating an anti-tumour response by the creation of a personalized vaccine consisting of autologous (neo)antigens $(1,2)$. This concept places radiotherapy (RT) in a different perspective, since this RT-induced in situ vaccine may be a key component in personalized immunotherapy (3).

Recently, we have shown that RT combined with L19-IL2, a stimulatory immunocytokine, can induce long-lasting cytotoxic T cell (4) and NK cell mediated (5) anti-tumour effects, depending on the tumour model. These findings are currently being investigated in a Phase I clinical study (NCT02086721) in our institute in patients with oligometastatic solid tumours (https://youtu.be/xHbwQuCTkRc). L19-IL2 contains the monoclonal antibody fragment L19, which recognizes the alternatively spliced extra-domain B (ED-B) of fibronectin, a marker associated with tumour angiogenesis. ED-B is present in newly formed vasculature structures of most solid tumours and absent in healthy tissues (with exception of tissues of female reproductive cycle and embryonic tissue), making it an interesting tumour targeting protein (6). The synergistic effect of RT combined with L19IL2 is however dependent on the radiation dose, i.e. decreases at lower RT dose (4). Increasing RT dose is a possible way to create optimal synergy with the immunostimulatory cytokine L19-IL2, but RT dose cannot be escalated unlimitedly because of normal tissue constraints (7).

Another possible and novel approach might be the addition of an immune checkpoint inhibitor that actively inhibits immune dampening pathways (8). One of the recent FDA (Food and Drug Administration) approved immune checkpoint inhibitors, anti-CTLA-4 (ipilimumab), has indeed shown ability to stimulate $T$ cell proliferation and cytokine production $(9,10)$, which resulted in encouraging durable tumour regression in a number of (metastatic) cancer patients (8). CTLA-4 is highly related to CD28 but has the opposite function. While CD28 signaling promotes T cell activation, CTLA-4 suppresses the $T$ cell response and has an immunoregulatory role (11). Both CD28 and CTLA-4 interact with CD80/CD86 on antigen presenting cells (12), and competing with CD28 for binding with shared ligands is one of the mechanisms that has been proposed to result in the inhibitory function of CTLA-4 (13-17). Blocking CTLA-4 using immune checkpoint inhibitors can therefore shift the balance into favouring CD28-mediated $\mathrm{T}$ cell activation, a concept of 'releasing the brakes'. Moreover, recent reports show that even greater success can be achieved by combining checkpoint inhibitors with other inhibitors such as against the PD-1/PD-L1 axis, tumour vaccines or radiotherapy (18-22).

Because ipilimumab is known to prolong the anti-tumour immune response and has shown to be even more successful in combination with RT, we hypothesized that we 
can observe synergistic anti-tumour effects by combining low dose RT and L19-IL2 (bimodal treatment) with the immune checkpoint inhibitor of CTLA-4.

\section{RESULTS}

\section{Anti-CTLA4 antibody decreases anti-tumour efficacy of the bimodal treatment}

To investigate if CTLA-4 is a possible target to increase the RT and L19-IL2 induced antitumour immune response, we first confirmed the expression of CTLA-4 on T cells in blood from L19-IL2 treated mice. Flow cytometric analysis (FACS) on peripheral blood revealed a higher percentage $(p=0.03)$ of circulating CTLA-4 expressing cytotoxic $\left(C D 8^{+}\right)$ T cells [3.9\%, 1.8-8.9] in L19-IL2 treated animals as compared with vehicle control mice [0.8\%, 0.6-1.5] (figure 1A), though the total amount of $\mathrm{CD}^{+} \mathrm{T}$ cells did not differ significantly $(p=0.25)$. Since CTLA-4 expression is known to downregulate $T$ cell function (23) and the anti-tumour effect of RT + L19-IL2 depends highly on these cytotoxic T cells (24), we investigated a trimodal therapy consisting of RT (2Gy), L19-IL2 and anti-CTLA-4. We evaluated the T4xSV for all treatment groups in the high ED-B expressing C51 mouse colon carcinoma model. Any combination treatment with RT, shows an increased T4XSV compared to vehicle control (at least $\mathrm{p}<0.03$ ) or monotherapy $(p<0.01) . R T+$ L19-IL2 significantly increased T4xSV (10.0 [5.9-21.0] days) compared to any other treatment schedule $(p<0.001)$ and resulted in a long-lasting cure in 5 out of 18 tumours (figure $1 \mathrm{~B}$ ). The combination of RT + L19-IL2 + anti-CTLA4 increased T4xSV $(p<0.005)$ as compared with all other treatments, except for RT + L19-IL2. The triple combination strikingly reduced ( $p=0.04$ ) T4xSV (8.0 [6.3-22.0 days) and cure rate (8\%) as compared with RT + L19-IL2 (Fig. 1B).

\section{Regulatory T cells in blood do not differ between bi- and trimodal treatment}

Several human and mouse studies have shown that the use of anti-CTLA-4 antibodies can lead to the enhancement of regulatory (Foxp $3^{+} \mathrm{CD} 25^{+} \mathrm{CD} 4^{+}$) $\mathrm{T}$ cell (Tregs) proliferation and overall frequency $(25,26)$. Therefore, we investigated if addition of anti-CTLA-4 to RT + L19-IL2 treatment could increase the amount of circulating Tregs as possible explanation for the decreased therapeutic efficacy of the trimodal treatment as compared with the RT + L19-IL2 bimodal treatment (figure 1C). The total amount of circulating CD8+ T cells did not differ between all treatment groups (data not shown). Anti-CTLA-4 treatment does not increase the amount of circulating Tregs in agreement with the lack of therapeutic efficacy (figure 1b). Although the trimodal treatment was less effective compared to the curative RT + L19-IL2 therapy, no significant differences in Tregs expression (Fig. 1c) and Treg/Thelper ratio (data not shown) were observed. Furthermore, we have found that L19-IL2 treatment enhances circulating Tregs, L19-IL2 
+ anti-CTLA-4 (8.9\% [7.7-10.7]) and 2Gy + L19-IL2 + anti-CTLA-4 treatment (7.3\% [6.09.2]) show a significantly higher percentage of Tregs as compared with anti-CTLA-4 (3.0\% [1.1-4.1], $p=0.002)$ and 2Gy + anti-CTLA4 (4.6\% [4.1-5.7], $p=0.004)$, respectively.

A
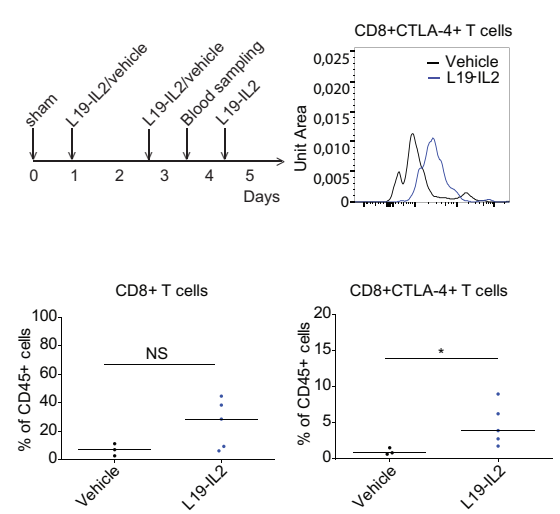

C
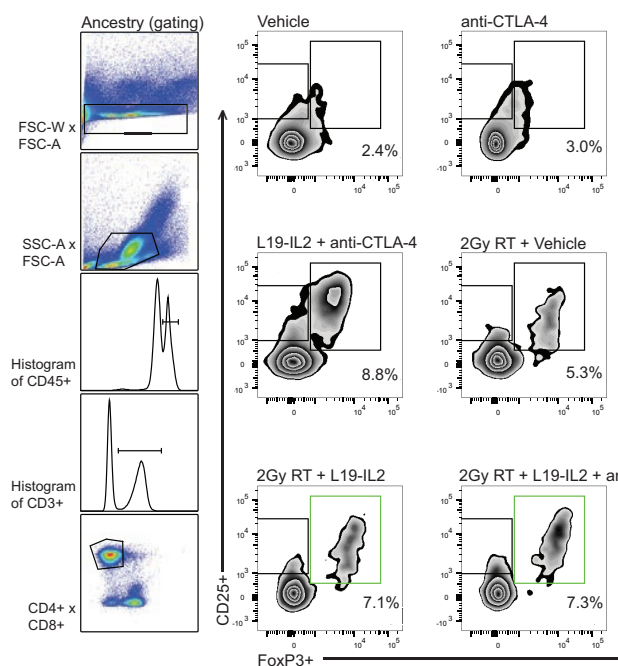

B
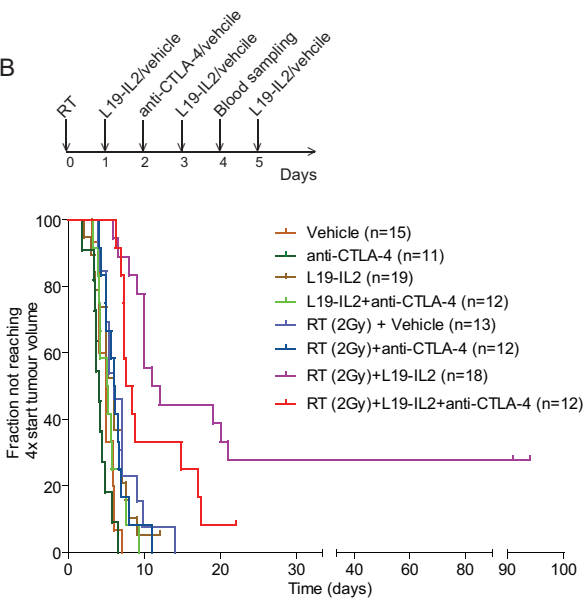

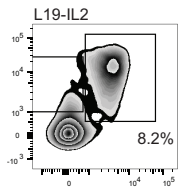

2Gy RT + anti-CTLA-4
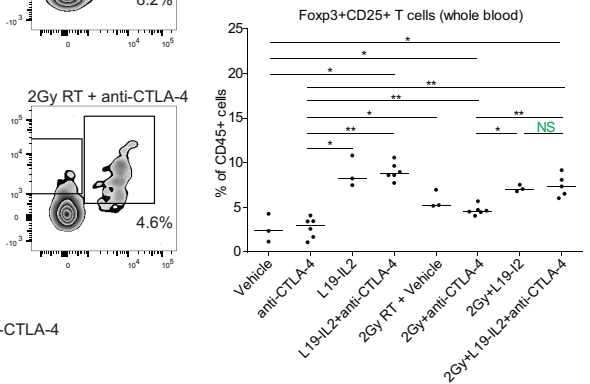

2Gy RT + L19-IL2 + anti-CTLA-4

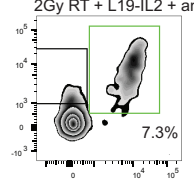

Figure 1: Growth delay trimodal treatment and peripheral regulatory T cells. (A) Schedule used for blood sampling and quantification of $\% \mathrm{CD} 8^{+}$and $\mathrm{CD} 8^{+} \mathrm{CTLA}-4^{+}$of total peripheral $\mathrm{CD} 45^{+}$immune cells. (B) Schedule used and fraction of tumours not reaching 4 times start tumour volume. (C) Representative gating and quantification of Foxp $3^{+} \mathrm{CD} 25^{+} T$ cells of total peripheral $C D 45^{+}$immune cells. ${ }^{*} p<0.05,{ }^{* *} p<0.01$. 
Recently, it has been shown that tumours have the ability to escape RT + anti-CTLA-4 mediated immune killing via the upregulation of PD-L1 (19). Therefore, we next investigated the expression of PD-L1 in tumours from the different treatment arms. Immunofluorescent stainings revealed that all tumour cells indeed express PD-L1 and tumours isolated from mice who received the trimodal treatment tend to have a higher mean PD-L1 expression (1150 \pm 91) as compared with the vehicle (1091 \pm 124$)$, RT + L19IL2 (1023 \pm 192$)$ and the RT treated (923) tumours (figure 2), which requires further confirmation.
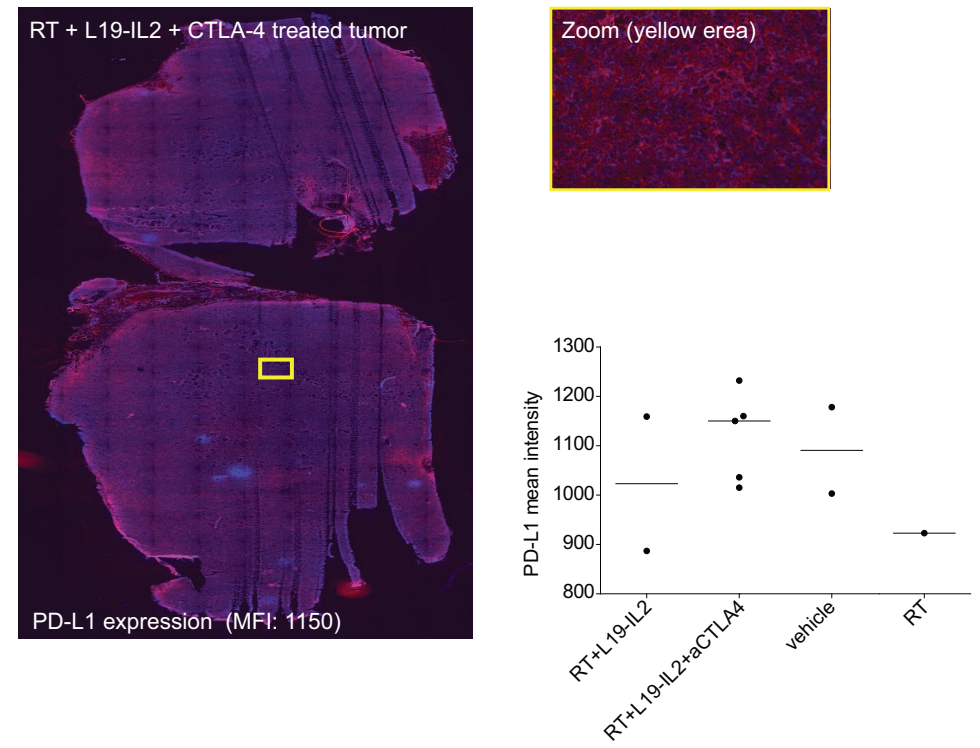

Figure 2: PD-1L expression in tumour tissues. Representative image of PD-1L expression in tumour and quantification of PD-L1 expressing among different treatment groups. (in blue: Dapi, PD-L1: in red).

\section{DISCUSSION}

Previously we have shown that the RT + L19-IL2 bimodal therapy can induce long-lasting curative cytotoxic $T$ cell responses, depending on the tumour model and the delivered RT dose (24) and NK cell mediated responses (5). Addition of an immune checkpoint inhibitor (8) might enhance the therapeutic effect of this combination treatment, since unlimitedly increasing the RT dose is not recommended because of normal tissue constraints (7). Here we investigated the therapeutic potential of a trimodal treatment approach, combining RT and L19-IL2 with anti-CTLA-4 (ipilimumab). 
Although the role of CTLA-4 is controversial $(27,28)$, anti-CTLA-4 therapy has shown encouraging durable tumour regression in a number of (metastatic) cancer patients (8) and recent data demonstrate that even greater success can be achieved by combining various checkpoint inhibitors with each other, with tumour vaccines or radiotherapy (18-22). Indeed, RT can improve the response rate of anti-CTLA-4 (29). The combination of anti-CTLA-4 with L19-IL2 also resulted in high cure rates in a preclinical study (30) and durable but no synergistic effects (due to high IL2 response rates) could be observed in a clinical trial combining anti-CTLA-4 with IL2 administration (31).

L19-IL2 treatment resulted in a higher percentage of circulating CTLA-4 expressing T cells (figure 1A) and it has been shown that RT can improve anti-CTLA-4 efficacy (29, 32). Therefore, we hypothesized that the addition of anti-CTLA-4 could further increase the RT + L19-IL2 induced anti-tumour immune response. In this study, the trimodal treatment (RT + L19-IL2 + anti-CTLA-4) increases the time to reach 4 times starting volume as compared with all other schedules, except for the bimodal treatment. Strikingly, we observed a reduced cure rate in the trimodal treatment group compared to RT + L19-IL2. Since it is known that the upregulation of CTLA-4 is a major immune dampening feature in order to control the duration and amplitude of a physiological immune response with minimal collateral tissue damage (8), our chosen time point of anti-CTLA-4 administration (day 2) might be too early to prolong and enhance the immune response.

Since CTLA-4 is not only expressed on exhausted cytotoxic T cells but also on Tregs (33), and the use of anti-CTLA-4 antibodies can lead to the enhancement of Treg proliferation $(25,26)$, we first investigated if the reduced anti-tumour effect could be explained by an increase in circulating Tregs. Our results indicate that anti-CTLA-4 treatment has no effect on circulating Tregs when added as additional therapy and is therefore not the underlying reason for the reduced anti-tumour effect of the trimodal therapy. Additionally, L19-IL2 monotherapy tends to increase the expression of circulating Tregs to a similar extent as high dose IL2 as described before (34). However, previously we (24) and others (6) have shown that L19-IL2 shows better anti-tumour responses as compared with systemic IL2 treatment, justifying the use of L19-IL2 over IL2 treatment.

The upregulation of a tumour immune escape mechanism can be another possible explanation for the observed resistance after anti-CTLA-4 administration. Recently, it was shown that tumours (melanoma) can escape anti-CTLA-4 based therapies by the upregulation of PD-L1 (19). Our preliminary results indeed show that PD-L1 is expressed in the tumour model used, and therefore it is possible that the PD-L1/PD-1 axis is involved as immunosuppressive mechanism. Furthermore, we observed a trend towards higher PD-L1 expression in the trimodal treatment; however, these data need to be confirmed at a time point shorter after RT + L19-IL2 treatment initiation using larger sample size, which is ongoing in our laboratory. Based on recent studies (19) and 
our first results, the rational of combining anti-PD-1 instead of anti-CTLA4 with RT + L19IL2, seems to be a promising combination.

Furthermore, in the present study we administrated one dose of ipilimumab at day 2. This schedule was based on our previous used schedule (RT day 0, L19-IL2 day 1, 3, 5) (24) and the anti-tumour activity observed in another preclinical study administrating ipilimumab one day after L19-IL2 injections (30). However, since we observe a worse anti-tumour effect using the trimodal treatment, the suboptimal timing of this checkpoint inhibitor, thereby contrarily inhibiting cells necessary for the optimal antitumour immune response, might be another possible explanation for this observed worse anti-tumour response after anti-CTLA-4 administration.

Based on these findings we conclude that anti-CTLA-4 treatment was not capable of increasing the RT + L19-IL2 anti-tumour effect. Upregulation of PD-L1 or PD-1 by antiCTLA-4 treatment might be an explanation of the observed resistance occurred in the trimodal treatment, but timing of the anti-CTLA-4 treatment might also explain the lack of therapeutic benefit.

\section{MATERIAL AND METHODS}

\section{Tumour cell lines/ reagents/ antibodies for in vivo studies}

Exponentially growing C51 mouse colon carcinoma cell lines (kindly provided by Philogen S.p.A., Siena, Italy) were cultured in Dulbecco's Modified Eagle Medium (DMEM), supplemented with $10 \%$ fetal calf serum (FCS) in a humidified $5 \% \mathrm{CO}_{2}$ chamber at $37^{\circ} \mathrm{C}$. The L19-IL2 immunocytokine (Philogen S.p.A.) was diluted with sterile phosphate buffered saline (PBS, Lonza) to concentrations of $200 \mu \mathrm{g} / \mathrm{ml}$.

\section{in vivo experiments}

All experiments were performed in accordance with local institutional guidelines for animal welfare and were approved by the Animal Ethical Committee of the University of Maastricht. To induce tumours, approximately 8 weeks old immune competent Balb/c (Harlan Laboratories) mice were subcutaneously injected with the ED-B positive (24) 1.5 x $10^{6}$ syngeneic C51 tumour cells, suspended in Basement Membrane Matrix (MatrigelTM, BD Biosciences), during fixation of the mice to obtain a xenograft tumour. Injected cells were screened for mice pathological organisms (QM MAP test). During the experiment, subcutaneously implanted tumours were measured several times a week (depending on speed of tumour growth). For this measurement we used a Vernier caliper measuring three orthogonal diameters $(A \times B \times C)$, which were corrected for thickness of the skin. Tumour volume was calculated using the following formula: $A \times B \times$ 
$\mathrm{C} \times \pi / 6$. Upon an average tumour volume of $200-250 \mathrm{~mm}^{3}$, animals were anaesthetized using ketamine/xylazine mix i.p. (100 mg/kg $+10 \mathrm{mg} / \mathrm{kg}$ ) and tumours were irradiated with a single dose RT dose of 2Gy (which was based on our previous RT dose-dependent treatment effects (24) and in order to create a window of opportunity for anti-CTLA-4) on day 0 , followed by systemic therapy (vehicle PBS / L19-IL2 20 $\mathrm{g}$ ) on day 1, 3 and 5 and (vehicle PBS / anti-CTLA-4 $10 \mathrm{mg} / \mathrm{kg}$ i.v) on day 2. All i.v. injections occur in the tail vein using a tail fixator. Tumour growth and treatment toxicity (based on body-weight) were monitored until reaching 4 times the volume at irradiation time (T4xSV).

\section{Flow Cytometric analysis}

Flow cytometric analysis was performed on blood samples. Blood samples were collected via punction of the vena saphena of L19-IL2 and vehicle treated mice at day 4. Red blood cells lysis was performed using RBC lysis buffer (eBioscience, San Diego, CA). Cells were incubated with FC-block CD16/CD32 (clone 2.4G2; BD Biosciences, San Jose, CA) and stained with a combination of following the antibodies for cell surface markers: antiCD45-PerCP (30-F11), anti-CD152 (CTLA-4)-Brilliant Violet 421 (UC10-4B9), anti-279 (PD1)-PE-Cy7 (RMP1-30) (Biolegend); anti-CD3e-FLUO 450 (145-2c11), anti-CD4-FITC (RM45), anti-CD8a-V500 (53-6.7) (BD Biosciences), CD25-APC (PC61.5) (eBioscience). For intracellular staining, FC blockade and cell surface markers staining was performed, cells were washed with a fixation/permeabilization working solution (eBioscience) according to manufacter's guidelines and then stained with anti-mouse/rat FOXp3-PE staining set (FJK-16s; eBioscience). Eight-colour flow cytometric analysis was performed with a FACSCanto II instrument (BD Biosciences). Data were analyzed with FACSDiva v6.1.2 (BD Biosciences) and FlowJo v10.0.8 (Tree Star) software. The total $\mathrm{CD} 45^{+}$immune cells were selected from the viable population of cells (filtered for debris and doublets) for further sub-classification according to the strategy described previously (24).

\section{Immunofluorescence}

Tumour sections $(7 \mu \mathrm{m})$ from the central part of a tumour were fixed with acetone $\left(4^{\circ} \mathrm{C}\right)$, rehydrated in phosphate buffered saline (PBS), incubated with normal goat serum followed by incubation with the primary rat anti-mouse PD-L1 antibody (1:100; Abcam) overnight at $4^{\circ} \mathrm{C}$ and secondary goat anti-rat Alexa594 (1:500, Invitrogen) for 1 hour at room temperature. Sections were mounted using fluorescent mounting medium (DakoCytomation). All sections we stained on the same day. Whole tumour crosssections were scanned using an Olympus BX51WI microscope equipped with a Hamamatsu EM-CCD C9100 digital camera, a motorized stage (Ludl Mac 2000) and 10fold objective. The same exposure time was used for all recordings. After scanning, sections were stained with hematoxylin and eosin for the identification of the viable tumour compartment by morphological criteria. Mean intensity of PD-L1 staining was 
measured in a blinded manner in each tumour cross-section in viable tumour areas, which was manually delineated excluding cutting and processing artefacts.

\section{ACKNOWLEDGEMENTS}

Authors acknowledge financial support from ERC advanced grant (ERC-ADG-2015, $\mathrm{n}^{\circ}$ 694812 - Hypoximmuno), the European Program H2020-2015-17 (ImmunoSABR - $n^{\circ}$ 733008) and Kankeronderzoekfonds Limburg from the Health Foundation Limburg. 


\section{REFERENCES}

1. Demaria S, Golden EB, Formenti SC. Role of Local Radiation Therapy in Cancer Immunotherapy. JAMA Oncol. 2015;1(9):1325-32.

2. Schumacher TN, Schreiber RD. Neoantigens in cancer immunotherapy. Science. 2015;348(6230):69-74.

3. Formenti SC, Demaria S. Radiation therapy to convert the tumor into an in situ vaccine. Int J Radiat Oncol Biol Phys. 2012;84(4):879-80.

4. Zegers CM, Rekers NH, Quaden DH, Lieuwes NG, Yaromina A, Germeraad WT, et al. Radiotherapy combined with the immunocytokine (L19-IL2) provides long-lasting anti-tumor effects. Clinical cancer research : an official journal of the American Association for Cancer Research. 2014.

5. Rekers NH, Zegers CM, Yaromina A, Lieuwes NG, Biemans R, Senden-Gijsbers BL, et al. Combination of radiotherapy with the immunocytokine L19-IL2: Additive effect in a NK cell dependent tumour model. Radiotherapy and oncology : journal of the European Society for Therapeutic Radiology and Oncology. 2015.

6. Carnemolla B, Borsi L, Balza E, Castellani P, Meazza R, Berndt A, et al. Enhancement of the antitumor properties of interleukin-2 by its targeted delivery to the tumor blood vessel extracellular matrix. Blood. 2002;99(5):1659-65.

7. Trani D, Yaromina A, Dubois L, Granzier M, Peeters SG, Biemans R, et al. Preclinical Assessment of Efficacy of Radiation Dose Painting Based on Intratumoral FDG-PET Uptake. Clinical cancer research : an official journal of the American Association for Cancer Research. 2015;21(24):5511-8.

8. Pardoll DM. The blockade of immune checkpoints in cancer immunotherapy. Nature reviews Cancer. 2012;12(4):252-64. PMCID: 4856023.

9. Blair PJ, Riley JL, Levine BL, Lee KP, Craighead N, Francomano T, et al. CTLA-4 ligation delivers a unique signal to resting human CD4 $T$ cells that inhibits interleukin-2 secretion but allows $B C l-X(L)$ induction. Journal of immunology. 1998;160(1):12-5.

10. Krummel MF, Allison JP. CD28 and CTLA-4 have opposing effects on the response of $T$ cells to stimulation. The Journal of experimental medicine. 1995;182(2):459-65. PMCID: 2192127.

11. Lenschow DJ, Walunas TL, Bluestone JA. CD28/B7 system of T cell costimulation. Annual review of immunology. 1996;14:233-58.

12. Sansom DM. CD28, CTLA-4 and their ligands: who does what and to whom? Immunology. 2000;101(2):169-77. PMCID: 2327073.

13. Bour-Jordan H, Esensten JH, Martinez-Llordella M, Penaranda C, Stumpf M, Bluestone JA. Intrinsic and extrinsic control of peripheral T-cell tolerance by costimulatory molecules of the CD28/ B7 family. Immunological reviews. 2011;241(1):180-205. PMCID: 3077803.

14. Rudd CE, Taylor A, Schneider H. CD28 and CTLA-4 coreceptor expression and signal transduction. Immunological reviews. 2009;229(1):12-26. PMCID: 4186963.

15. Teft WA, Kirchhof MG, Madrenas J. A molecular perspective of CTLA-4 function. Annual review of immunology. 2006;24:65-97.

16. Walker LS, Sansom DM. The emerging role of CTLA4 as a cell-extrinsic regulator of T cell responses. Nat Rev Immunol. 2011;11(12):852-63.

17. Wing K, Yamaguchi T, Sakaguchi S. Cell-autonomous and -non-autonomous roles of CTLA-4 in immune regulation. Trends in immunology. 2011;32(9):428-33.

18. Leavy O. Tumour immunology: A triple blow for cancer. Nat Rev Immunol. 2015;15(5):265.

19. Twyman-Saint Victor C, Rech AJ, Maity A, Rengan R, Pauken KE, Stelekati E, et al. Radiation and dual checkpoint blockade activate non-redundant immune mechanisms in cancer. Nature. 2015;520(7547):373-7. PMCID: 4401634.

20. Postow MA, Callahan MK, Barker CA, Yamada Y, Yuan J, Kitano S, et al. Immunologic correlates of the abscopal effect in a patient with melanoma. N Engl J Med. 2012;366(10):925-31. PMCID: 3345206.

21. Vanneman M, Dranoff G. Combining immunotherapy and targeted therapies in cancer treatment. Nature reviews Cancer. 2012;12(4):237-51. PMCID: 3967236. 
22. Wada S, Jackson CM, Yoshimura K, Yen HR, Getnet D, Harris TJ, et al. Sequencing CTLA-4 blockade with cell-based immunotherapy for prostate cancer. J Transl Med. 2013;11:89. PMCID: 3666941.

23. Jiang $Y$, Li $Y$, Zhu B. T-cell exhaustion in the tumor microenvironment. Cell death \& disease. 2015;6:e1792. PMCID: 4669840.

24. Zegers CM, Rekers NH, Quaden DH, Lieuwes NG, Yaromina A, Germeraad WT, et al. Radiotherapy combined with the immunocytokine L19-IL2 provides long-lasting antitumor effects. Clinical cancer research : an official journal of the American Association for Cancer Research. 2015;21(5):1151-60.

25. Kavanagh B, O'Brien S, Lee D, Hou Y, Weinberg V, Rini B, et al. CTLA4 blockade expands FoxP3+ regulatory and activated effector CD4+ T cells in a dose-dependent fashion. Blood. 2008;112(4):1175-83. PMCID: 2515138.

26. Tang AL, Teijaro JR, Njau MN, Chandran SS, Azimzadeh A, Nadler SG, et al. CTLA4 expression is an indicator and regulator of steady-state CD4+ FoxP3+ T cell homeostasis. Journal of immunology. 2008;181(3):1806-13. PMCID: 2683757.

27. Nakamura K, Kitani A, Strober W. Cell contact-dependent immunosuppression by CD4(+)CD25(+) regulatory $T$ cells is mediated by cell surface-bound transforming growth factor beta. The Journal of experimental medicine. 2001;194(5):629-44. PMCID: 2195935.

28. Read S, Malmstrom V, Powrie F. Cytotoxic T Iymphocyte-associated antigen 4 plays an essential role in the function of $\mathrm{CD} 25(+) \mathrm{CD} 4(+)$ regulatory cells that control intestinal inflammation. The Journal of experimental medicine. 2000;192(2):295-302. PMCID: 2193261.

29. Vanpouille-Box C, Pilones KA, Wennerberg E, Formenti SC, Demaria S. In situ vaccination by radiotherapy to improve responses to anti-CTLA-4 treatment. Vaccine. 2015;33(51):7415-22. PMCID: 4684480.

30. Schwager K, Hemmerle T, Aebischer D, Neri D. The Immunocytokine L19-IL2 Eradicates Cancer When Used in Combination with CTLA-4 Blockade or with L19-TNF. J Invest Dermatol. 2012.

31. Maker AV, Phan GQ, Attia P, Yang JC, Sherry RM, Topalian SL, et al. Tumor regression and autoimmunity in patients treated with cytotoxic T lymphocyte-associated antigen 4 blockade and interleukin 2: a phase I/II study. Annals of surgical oncology. 2005;12(12):1005-16. PMCID: 1473970.

32. Verbrugge I, Hagekyriakou J, Sharp LL, Galli M, West A, McLaughlin NM, et al. Radiotherapy increases the permissiveness of established mammary tumors to rejection by immunomodulatory antibodies. Cancer research. 2012;72(13):3163-74

33. Walker LS. Treg and CTLA-4: two intertwining pathways to immune tolerance. Journal of autoimmunity. 2013;45:49-57. PMCID: 3989116.

34. Ahmadzadeh M, Rosenberg SA. IL-2 administration increases CD4+ CD25(hi) Foxp3+ regulatory T cells in cancer patients. Blood. 2006;107(6):2409-14. PMCID: 1473973. 
Chapter

General discussion and future perspectives 
The overall aim of this dissertation was to investigate the possibility of transforming the effects of local radiotherapy (RT) into a systemic treatment using the immunocytokine L19-IL2. This is of major importance, since RT is usually applied locally or locoregionally, therefore (micro)metastases outside the radiation field will be missed and have the opportunity to grow. However, it is not only of major importance, it is also of enormous interest. It is known that RT can initiate an anti-tumour immune response (1). Therefore the possibility of transforming and improving this anti-tumour immune response using an immunomodulatory approach, holds great potential for creating a personalized, multifaceted 'medicine'. This medicine, the anti-tumour immune response itself, will contain elements of a patient's tumour and has therefore the potential to target this heterogeneous disease in the same heterogeneous manner, with a curative intent.

\section{TOWARDS A MULTI-FACETED CORPUS-DRIVEN TREATMENT - THE FIRST STEPS}

In chapter 3 we hypothesized that the combination of RT (10Gy) with the immunocytokine L19-IL2 causes a synergistic anti-tumour effect on irradiated tumours dependent on the expression of ED-B. Therefore, in this study, we assessed the therapeutic potential and related induced anti-tumour immune responses of this combination therapy in three different in vivo mouse tumour models: the 4T1 breast carcinoma, Lewis lung carcinoma (LLC), and the C51 colon carcinoma, which have respectively increasing expression levels of ED-B (2). The combination treatment showed no additional therapeutic improvement in the low/negative ED-B expressing model 4T1 and merely an additive effect was observed in the intermediate ED-B expressing LLC model. Importantly, highly synergistic effects with a cure rate of $75 \%$ were detected in the high ED-B expressing model C51 (2), in line with the higher L19-IL2 tumor targeting capacity when having more ED-B expression. ED-B is overexpressed in many solid tumors (3-7), making L19-IL2 applicable for the majority of cancers. Previous imaging and targeted (radio)immunotherapy studies have shown that L19 targets the tumour (vasculature) (8-10). Moreover, the administration of L19-IL2 alone or combined with chemotherapy (decarbazine), was safe and showed clinical activity in a phase I study in patients with melanoma or renal cell carcinoma $(11,12)$ and a phase II study investigating the efficacy of L19-IL2 combined with L19-TNF in stage III and IV melanoma patients is ongoing (NCT02076633).

Moreover, we showed that the percentage of tumour infiltrating $\mathrm{CD}^{+}$cytotoxic $T$ cells are higher in ED-B expressing tumours treated with RT + L19-IL2 and a CD8 ${ }^{+}$depletion experiment provided direct evidence that the complete remission of C51 tumours is causally related to these immune cells (2). These data are in line with other studies, in which KS-IL2 (targeted to epithelial cell adhesion molecule) combined with 
radiofrequency ablation in a murine colon adenocarcinoma (CT26) showed increased growth suppression associated with a larger proportion of $\mathrm{CD} 4^{+}$and $\mathrm{CD} 8^{+} \mathrm{T}$ cells (13). Moreover, the therapeutic effect of F8-IL2 and F16-IL2 (recognizing ED-A/ED-B domains of fibronectin and the $A 1$ domain of tenascin- $C$, respectively) was also shown to be mediated by $\mathrm{CD}^{+} \mathrm{T}$ (and NK) cells in an in vivo AML model (14). To investigate the dependency of the synergy between RT and L19-IL2 on the RT dose, we additionally investigated a single RT dose of 5 and $2 \mathrm{~Gy}$. Lower RT doses resulted in a reduced number of tumour cures (2). In line with our findings, in vitro studies have shown that the immune stimulating effects of radiation are dose-dependent (15), suggesting that high RT doses induce more immunogenic effects. However, these effects are largely dependent on the complex composition of the tumour microenvironment (16). There is no real consensus about the optimal RT dose or regimen to induce the best immune initiating effects and therefore resulting in the optimal synergy between RT and an immunotherapeutic approach (17). Some studies support the use of a single high RT dose (30Gy), others show that a dose of $2 \mathrm{~Gy}$ or hypo-fractionated doses of 6 or $8 \mathrm{~Gy}$ are the best triggers to be used (18-20). These divergent results might be dependent on the tumour model used and might be explained by differences in tumour immunogenicity or composition of immune infiltrating cells at start of (RT) treatment (21). A clear readout system (i.e. biomarker) to select patients for a certain RT regimen, maximizing their personal 'in situ' vaccine and therefore creating the best anti-tumour immune response starting point, is in my opinion crucial. From there on, selection for the optimal immune modulation strategy and patient monitoring (on tumour and systemic level) should be performed. Based on our preclinical results described in chapter 3 (2) and clinical results proving the higher response rate of high-dose stereotactic body radiotherapy (SBRT) (22) with systemic IL2 in patients with metastatic melanoma or RCC (23) as compared with historical controls, we suggest that the use of SBRT is sufficient to release DAMPs and initiate the anti-tumour immune response. Since L19-IL2 is proven to be safe in patients, these findings are translated into a Phase I clinical safety study (NCT02086721) combining SBRT and L19-IL2 in patients with oligometastatic solid tumours, which is currently ongoing in our institute. So far, preliminary results of this trial indicate that the treatment combination is safe for the first dose level, accompanied with pain in the tumour region suggesting treatment efficacy.

Overall, these data prove that a synergy between RT and L19-IL2 exists, and that it is possible to develop curative anti-tumour immune-mediated effects in irradiated tumours under certain conditions including the presence of ED-B expression and cytotoxic $T$ cell infiltrate. In chapter 4 we place the current findings into a broader perspective and describe how our findings reported in chapter 3 (2) form the basis for following research (24). In my opinion, learning from responders, which reveals groundbreaking secrets, is the optimal way to move forward and to translate these secrets into usable future knowledge applicable to treat non-responders. Therefore, the 
following chapters are focusing on the 'winning medicine': the RT + L19-IL2 anti-tumour immune response triggered in Balb/c mice bearing ED-B positive C51 tumours. Because the major aim of this research is to create preclinical evidence for a multi-faceted systemic 'patient-driven' treatment, we discussed the utility of this experimental set-up towards further preclinical translational approaches. This chapter forms a little bridge, giving us a sneak-preview about what is coming: translating the research (this winning medicine) to other experimental set-ups which enables us to investigate the antitumour immune effect outside the RT field (25), long-term anti-tumour effects, i.e. immunological memory against the tumour cells, and the possibility of novel combinations, for example, with hypoxia activated prodrugs (26) and checkpoint inhibitors (27).

\section{EXPLORING THE OUT OF FIELD ANTI-TUMOUR EFFECTS}

Based on the results obtained in chapter 4 and since immune cells operate systemically, we hypothesized in chapter 5 that the RT + L19-IL2 mediated anti-tumour immune effect has long-lasting anti-tumour effects outside the RT field. Since T cells recognize tumour-associated (neo)antigens in order to effectively perform their cytotoxic activity (28), and RT is known to increase the tumour (neo)antigen expression (1, 29), it is a highly relevant question to test: tumours outside the RT field might be a less obvious target for these immune cells. Furthermore, the study creates insights into this systemic anti-tumour effect which is crucial when treatment will be translated to patients with (micro)metastases. Anti-tumour effects outside the RT field, a phenomenon known as the abscopal (ab - position away from and scopus - target) effect (30-34), solely caused by RT are rare (35). Moreover, since the effect has been observed over decades in multiple cancers (36-42) and because of this long-lasting potential, it has been established that the RT-induced abscopal effect is immune-mediated (43). In chapter 5, we are not solely investigating the RT-induced abscopal effect, but combine it with L19IL2, and therefore we propose another terminology for this 'extended' abscopal effect, namely the Out-of-Field Radiolmmune (OFRI) effect.

In chapter 5 we show for the first time that a single RT dose (15Gy) resulted in a curative OFRI effect when combined with L19-IL2 in 20\% of the non-irradiated macroscopic tumours. Two fractionated RT regimes ( $5 \times 2 \mathrm{~Gy}$ and $5 \times 5 \mathrm{~Gy}$ ) resulted additionally in an OFRI effect, though without curative results. The first immunological analyses, performed on day 4 of the treatment, revealed an increase in $\mathrm{CD}^{+} \mathrm{T}$ infiltration in tumours outside the RT field, suggesting the involvement of $\mathrm{CD} 4^{+} \mathrm{T}$ cells in the RT + L19-IL2-mediated OFRI effect. Depletion of $\mathrm{CD}^{+}{ }^{+}$T cells, starting on day 5 after $\mathrm{RT}$, resulted in faster tumour growth of non-irradiated tumours but this effect was not statistically significant. On the other hand, depletion of $\mathrm{CD}^{+} \mathrm{T}$ cells abrogated the OFRI 
effect of the combination therapy significantly. These data provide direct evidence that the growth delay and complete remission of $20 \%$ of the non-irradiated tumours is predominantly attributed to $\mathrm{CD}^{+}{ }^{+} \mathrm{T}$ cells. 'Immunoscore', a scoring system which classifies cancer based on tumour immune infiltrate, has prognostic value proven for several malignancies (44), but might not be useful in case of systemic (metastatic) cancer. Therefore, the OFRI effects against metastases (when tumour biopsy cannot be investigated) might be predicted using mathematical models in the near future. These models, which incorporate physiologic information about $\mathrm{T}$ cell trafficking in order to estimate the distribution of activated $T$ cells between metastatic lesions, may additionally offer insight into the dynamics of systemic disease $(45,46)$.

To investigate specificity of OFRI effect, we used another ED-B positive secondary CT26 tumour (47) and observed a similar growth delay of this mismatched tumour. However, the OFRI effect did not result in mismatch tumour cures, suggesting common expression of only a part of the (neo)antigens between these secondary tumours, CT26 and C51. Further investigation into the antigen-specific T cells responsible for the RT + L19-IL2 mediated OFRI effect should be performed in order to predict outcome, and we believe that the novel technique using peptide- $\mathrm{MHCl}$ multimers labeled with DNA barcodes is the most reliable and extensive way to continue this research (48).

Of great importance, we were able to show that part of the out-of-field tumour infiltrating $T$ cells have a regulatory $\left(\mathrm{Foxp}^{+} \mathrm{CD}^{2} 5^{+}\right.$) phenotype or express PD-1, resulting in the tolerance and exhaustion of these cells (49). The expression of PD-1 on T cells is identified as an inhibitory mechanism of immune responses $(19,50,51)$. Several PD-1 inhibitors, including nivolumab, have obtained FDA approval (27) and show very promising anti-tumour activity $(52,53)$. Because of the observed immunosuppressive $T$ cell phenotype of $T$ cells infiltrating out-of-field tumours, and the availability of highly promising FDA approved new therapies, a basis is formed towards an even better OFRI effect. This exhausted T cell phenotype as well as increased infiltration of regulatory $T$ cells was not observed inside irradiated primary tumours, suggesting that a single RT dose can directly shift the effector/regulatory balance into an effective immune response, which is in line with published data $(1,54)$. In contrast, fractionated $(5 \times 2 \mathrm{~Gy})$ RT increased PD-1 expressing T cells inside both irradiated and non-irradiated tumours. Our results suggest that the addition of a checkpoint inhibitor such as anti-PD-1 is therefore useful in case T cells express high levels of PD-1. Comparison between nonirradiated tumours of 15Gy + L19-IL2 and 5 x 5Gy + L19-IL2 treated primary tumours revealed that the single $\mathrm{RT}$ dose treated mice have a higher percentage of peripheral (blood) $\mathrm{CD}^{+} \mathrm{T}$ cells and a higher percentage of tumour infiltrating $\mathrm{CD} 8^{+} \mathrm{T}$ cells. Based on our previous studies showing that $\mathrm{CD}^{+} \mathrm{T}$ cells are causing the RT + L19-IL2 antitumour effect of irradiated tumours (chapter 3, (2)) and since we have shown that $T$ cells are crucial for a RT + L19-IL2 OFRI effect, differences in percentage of circulating $\mathrm{CD}^{+} \mathrm{T}$ cells and infiltrating $\mathrm{CD}^{+} \mathrm{T}$ cells may explain why single RT dose is a better 
inducer of a curative OFRI effect. These data suggest that it might be interesting to extent the novel technique using peptide- $\mathrm{MHCl}$ multimers labeled with DNA barcodes (48) with peptide-MHCII multimers, in order to create a full understanding of the T cell mediated OFRI effect in the future. It is known that the RT dose, schedule and technique can provoke a pleiotropy of systemic immune responses (1), and as discussed above, there is no uniform consensus about the optimal immune activating RT trigger. Several preclinical studies showed significant growth delay and complete remission outside the RT field when immunotherapy (anti-CTLA-4) was combined with fractionated RT but not with a large single RT dose $(18,55)$. In contrast, Lee et al. showed that a single RT dose (20Gy) initiated immune responses and tumour (including non-irradiated metastases) growth retardation when combined with immunotherapy (ad-LIGHT) (56). These effects were not observed when combined with fractionated RT ( $4 \times 5 G y)$, similar to our findings. Based on the curative results of the single RT dose as compared with fractionated RT, we conclude that one single dose of RT is the best trigger in combination with L19-IL2 to create maximum tumour control at distance for this tumour model.

Moreover, in this chapter we describe the capability of RT + L19-IL2 cured mice to develop long-lasting immunological memory against these tumours. This protective immunity was only formed when tumour-bearing mice were treated with RT + L19-IL2, showing that the tumour forms the basis for the 'in situ' vaccine that can eventually be translated into a direct (irradiated), indirect (non-irradiated) and long-lasting (rechallenged) anti-tumour immune responses. The expression of CD127 is a hallmark of primed $\mathrm{CD}^{+} \mathrm{T}$ cells to develop into long-lived memory cells (57-60), however blocking of CD127 did not result in a different tumour take and growth upon re-challenging, showing that this long-lasting RT + L19-IL2 induced effect is associated with, though not caused by CD127. In contrast, mice cured by surgery + L19-IL2 and high dose RT + vehicle developed significantly more tumours after re-challenge accompanied by elevated normal tissue toxicity levels within the high dose RT treatment group. These results clearly show that RT + L19-IL2 tumour cure is absolutely necessary to achieve long-lasting anti-tumour protection. Furthermore, we revealed a significant increase of $\mathrm{CD}_{4} 4^{+} \mathrm{CD}_{2} \mathrm{~L}^{+}$and $\mathrm{CD} 44^{+} \mathrm{CD} 62 \mathrm{~L}^{-}$expression on $\mathrm{CD}^{+} \mathrm{T}$ cells in the lymph nodes, indicative for central memory $T$ cells and effector $T$ cells, respectively $(58,61,62)$ in cured mice able to prevent tumour development. Therefore, we conclude that central memory and effector $T$ cells in the lymph node compartments are involved in the protection against new C51 tumour formation. The same mice show high expression of $\mathrm{CD}_{4} 4^{+} \mathrm{CD} 127^{+}$on splenic, lymph nodes and on circulating (blood) $\mathrm{CD} 8^{+} \mathrm{T}$ cells, which are classified as effector memory $T$ cells $(58,61,62)$. Since effector memory $T$ cells are significantly increased in the blood of mice able to reject tumour cells upon rechallenge, these immune cells are systemically associated with an optimal long-lasting 
protective effect and may serve as biomarker to identify patients who did not develop immunological memory and thus is at higher risk of tumour relapse.

\section{A ROLE FOR NK CELLS}

So far, we observed that the combination therapy of RT + L19-IL2 was T cell dependent. In chapter 6, we hypothesize that this combination therapy can also trigger a NK cell dependent anti-tumour response in a model (F9 teratocarcinoma) lacking $\mathrm{MHCl}$ expression, however positive for ED-B expression (63). Indeed, it is known from literature that NK cells (belonging to the innate immune system) play a crucial role in the anti-tumour activity of a wide variety of tumour cells with reduced or absent $\mathrm{MHCl}$ expression (64). In this chapter we observed no tumour growth delay when RT was delivered during the L19-IL2 treatment schedule (RT day 2, L19-IL2 day 0, 2, 4). However, using the same treatment schedule (RT day 0, L19-IL2 day 1, 3, 5) as described in previous chapters, the combination treatment showed a therapeutic gain. This might be explained by the fact that L19-IL2 treatment stimulates the proliferation/activation of NK cells, which are killed when the RT dose is delivered during immunotherapy. Indeed, L19-IL2 treatment alone did already result in a significant tumour growth delay and showed a significant increase of tumour infiltrating NK cells in our study (65). This is in line with literature showing that NK cells do not necessarily need prior immunization (in this study by RT) to actively perform their cytotoxic activity against tumours (66). Indeed, we show in this chapter for the first time that this therapeutic gain in the $\mathrm{MHCl}$ negative $\mathrm{F} 9$ teratrocacinoma is associated with the infiltration of NK cells. In agreement with published data, the efficacy of targeted IL2 therapy (F16-IL2, F8-IL2, L19-IL2 or NHS-IL2) can be attributed to NK cells (67-69), T cells (70-73), or a combination of both $(63,74)$. Findings of this chapter, clearly showing that the anti-tumour effect of the (RT +) L19-IL2 (combination) therapy is present in a $\mathrm{MHCl}$ negative tumour model, are of great importance. In clinical setting, tumours tend to have a heterogeneous expression of $\mathrm{MHCl}$ [4] and therefore NK cells might be a crucial backup plan in case activated $\mathrm{CD}^{+} \mathrm{T}$ cells have no or limited targets to recognize these tumour cells. However, reported data suggest that direct NK cell efficacy against solid human tumours is still questionable, due to the immunosuppressive tumour microenvironment, but their indirect effects shaping the tumour microenvironment may be favoring adaptive $T$ cell responses (75-77). Because we have observed in chapter 6 that RT + L19-IL2 results in a significant anti-tumour effect and an increase of tumour infiltrating $\mathrm{NK}$ cells in a $\mathrm{MHCl}$ negative tumour model, we provide evidence that this treatment has a stimulating/activating effect on NK cells. Therefore, in clinical setting, NK cells stimulated by L19-IL2 might additionally increase the cytotoxic potential of RT + L19-IL2 stimulated T cells targeting irradiated tumours. In other words, L19-IL2 stimulated NK cells may induce an anti-tumour response via its indirect tumour 
microenvironment shaping effects, though we have not found an increase in NK cells in $\mathrm{MHCl}$ positive tumours treated with the combination treatment (chapter 3, (2)). Furthermore, highly interesting recent findings demonstrate that circulating NK cells have a role in metastasis control (78) and that impaired NK cell activity in the blood circulation can be observed in several malignancies (79-81). Given the fact that RT + L19-IL2 can induce anti-tumour effects outside the RT field (i.e. the OFRI effect) as described in chapter 5, and this therapy is able to stimulate NK cells as described in this chapter (chapter 6), this might hold even more promise for (micro)metastatic tumour control.

In chapter 7 we describe our opinion as answer on a commentary written based on chapter 6 (82), which focus on where to place L19-IL2 in 'the age of the checkpoint inhibitors'. As described in the introduction of this thesis (chapter 1), checkpoint inhibitors are able to target the inhibitory pathways of cells from the adaptive immune system (83), and have shown revolutionary clinical results (84-87). Anti-CTLA4 (ipilimumab) and the anti-PD-1/PD-L1 axis inhibitors atezolimab, pembrolizumab and nivolumab are all examples of recent FDA approved immune checkpoint inhibitors (88). In chapter 7 we discuss the author's assumption that checkpoint inhibitors act on the modulation of an already present adaptive immune response (cytotoxic T cells) and IL2 based therapies are only able to trigger the innate immune system (NK cells). We agree with the statement that the ability of L19-IL2 to activate NK cells creates an interesting alternative for checkpoint inhibitors in case the cytotoxic T cell 'baseline' is insufficient, in case $\mathrm{MHCl}$ is lacking. Mechanisms of checkpoint inhibitors relay on the re-activation of already present but exhausted tumour (neo)antigen specific T cells, whereas L19-IL2 function relies on the activation/proliferation of these tumour specific $T$ cells. In other words, checkpoint inhibitors are able to get rid of the brake, and L19-IL2 is able to push the gas. Because of this different mode of action between both immunotherapeutic approaches, and because of the discussed immune initiating effects of RT, we believe and conclude in chapter 7 that long-lasting (memory effects) and OFRI anti-tumour effects can be reached when the right RT dose/schedule will be combined with the stimulating immunotherapeutic approach in order to result in an optimal immune response of adaptive origin. As described above, NK cells might shape the adaptive anti-tumour immune response, and in this perspective I believe the involvement (indirect effects) of NK cells might be crucial. In the same perspective, I believe the addition of checkpoint inhibitors might be crucial since they have the potential to prolong the RT + L19-IL2 induced anti-tumour effect by blocking inhibitory immune dampening pathways (88).

So far, we conclude that the first line treatment must consist of RT to create a personalized in situ vaccine and increase tumour immunogenicity, followed by L19-IL2 to stimulate the proliferation of tumour (neo)antigen specific T cells (and/or NK cells that target $\mathrm{MHCl}$ negative tumour cells and/or support cytotoxic $\mathrm{T}$ cells). These activated cytotoxic T cells have the potential to target tumour cells outside the RT field 
(OFRI effect). As described above, $T$ cells have the possibility to activate several inhibitory pathways in order to maintain self-tolerance and to be able to control the duration and amplitude of a physiological immune response with minimal collateral tissue damage (88). This is a process researchers want to minimize, since these inhibitory pathways also stop the anti-tumour immune response (i.e. exhausted T cell phenotype). When these specific T cells become exhausted, they often express CTLA-4 and/or PD-1 immune downregulating molecules as described in chapter 1 . Because of these general characteristics, we next investigated the possibility to further increase the RT + L19-IL2 mediated immune response by the addition of anti-CTLA-4 (ipilimumab).

\section{TOWARDS TRIMODAL TREATMENT?}

In chapter 3 we have described that the RT + L19-IL2 bimodal therapy can result in longlasting curative cytotoxic $T$ cell responses, depending on the tumour model and the delivered RT dose (2) and additive NK cell associated responses (65) in $\mathrm{MHCl}$ negative tumours, as described in chapter 6 . Additionally we have shown in chapter 5 that increasing RT dose to $15 \mathrm{~Gy}$ resulted in a $100 \%$ cure rate when combined with L19-IL2. Instead of increasing the RT dose, combining the low dose RT + L19-IL2 treatment with a checkpoint inhibitor, may also increase therapeutic potential (88). The unlimited increase of the RT dose is not clinically feasible because of normal tissue constraints. In chapter 8 we investigated the therapeutic potential of a trimodal treatment approach, combining RT and L19-IL2 with anti-CTLA-4 (ipilimumab). We hypothesized that we can observe synergistic anti-tumour effects by combining low dose RT and L19-IL2 with the immune checkpoint inhibitor of CTLA-4. Indeed, we have shown in this study that treatment with L19-IL2 resulted in CTLA-4 expressing T cells, making this a promising target for further improvement. In chapter 8 we showed that the trimodal treatment (RT + L19-IL2 + anti-CTLA-4) prolongs growth delay as compared with all other schedules, except the bimodal treatment. Strikingly, we observed a reduced cure rate in the bimodal treatment when anti-CTLA-4 was added, which was not attributed to an increase in regulatory $T$ cells as described before $(89,90)$. Recently, it has been shown that tumours (melanoma) can escape anti-CTLA-4 based therapies by the upregulation of PD-L1 (91) and our preliminary results show indeed that PD-L1 is expressed in the tumour cells, and therefore it is possible that the PD-L1/PD-1 axis is involved as immunosuppressive mechanism. Furthermore, we only administrated one dose of ipilimumab at one time point (day 2). This schedule was based on our previously used schedule (RT day 0, L19-IL2 day 1, 3, 5) and the promising anti-tumour activity observed in another preclinical study administrating ipilimumab one day after L19-IL2 injections (92). However, the latter study administrated multiple ipilimumab injections, and therefore additional benefit of the trimodal treatment as compared with RT + L19-IL2 therapy. However, we observed a worse anti-tumour effect using the trimodal 
treatment. Therefore, the suboptimal timing of this checkpoint inhibitor, thereby contrarily inhibiting cells necessary for the optimal anti-tumour immune response, might be a possible explanation for this observed worse anti-tumour response after anti-CTLA-4 administration.

In conclusion, this dissertation showed the possibility of increasing a RT initiated antitumour immune response using the immunocytokine L19-IL2 in a $\mathrm{CD}^{+}$and $\mathrm{NK}^{+}$cell dependent manner, dependent on the model. It shows the possibilities to cause tumour regression and cure outside the RT field (OFRI effect) in a T cell dependent manner. It clearly shows the long-lasting activity of this stimulated immune response, which is even able to provide protection against new tumour formation months after the termination of this treatment. The combination treatment of RT with L19-IL2 provides the immune system with all the necessary tools and has therefore great potential to target metastatic disease, with a curative intent.

\section{FUTURE PERSPECTIVES - THIS DISSERTATION}

The future of RT combined with L19-IL2 bimodal treatment is highly promising. Figure 1 summarizes several future directions that can lead to a greater success of this approach. Based on the results described in chapter 3, a phase I clinical trial is initiated which is currently ongoing as described above (animation available: https://youtu.be/xHbwQuCTkRc) and a phase II clinical trial has been approved (animation available: https://youtu.be/6wDE6RkrikA). The phase II study will investigate the combination of SBRT and L19-IL2 in patients with non-small cell lung cancer (NSCLC). Using peptide- $\mathrm{MHCl}$ multimers labeled with individual DNA barcodes (48), we will be able to identify neo-epitope-directed T cell reactivity. This latter technique will give us important insights into the $T$ cell reactivity against tumour (neo)antigens, opening doors to create profiles of patients based on their anti-tumour immune response. This will form the basis for a new biomarker approach, the most important future step in oncology research. Furthermore, based on the results described in chapter 5, were we found the presence of immunosuppressive T cells (PD- $\left.1^{+}\right)$infiltrating tumours outside the RT field, we applied for a KWF grant in which we will establish the preclinical proof-of-concept of the therapeutic impact of a trimodal treatment for metastatic cancer (animation available: https://youtu.be/7ckZeWWyhts). This trimodal therapy will consist of RT, L19-IL2 and nivolumab (anti-PD-1). In addition, we will identify immune-specific blood and tissue biomarkers that can evaluate therapy response and OFRI effects. Novel preclinical findings will be translated to a new phase I clinical study in stage IV NSCLC patients, investigating safety as primary and overall survival as secondary endpoints respectively. Moreover, it will be interesting to investigate the T cells (CD44 ${ }^{+} \mathrm{CD} 127^{+}$, i.e. effector memory cells) described in chapter 5 
in blood of patients from the phase II study (or from blood of responders vs nonresponders from another clinical study). Effector memory $\mathrm{T}$ cell reactivity against tumour (neo)antigens might hold even more promise to identify (long-term) responders during or directly after treatment, which enables us eventually to classify patients into 'Iong-term responders' or 'non-long-term responders', and adapt therapy for the latter group. The role of NK cells in this anti-tumour immune response also warrants further research (chapter 6), and it might be of great value to investigate the effects of RT + L19-IL2 in a tumour model with a heterogeneous expression of $\mathrm{MHCl}$ and assess to what extent $\mathrm{NK}$ cells directly target $\mathrm{MHCl}$ negative tumour cells or indirectly support $\mathrm{CD}^{+} \mathrm{T}$ cell mediated responses, as described previously (75-77).

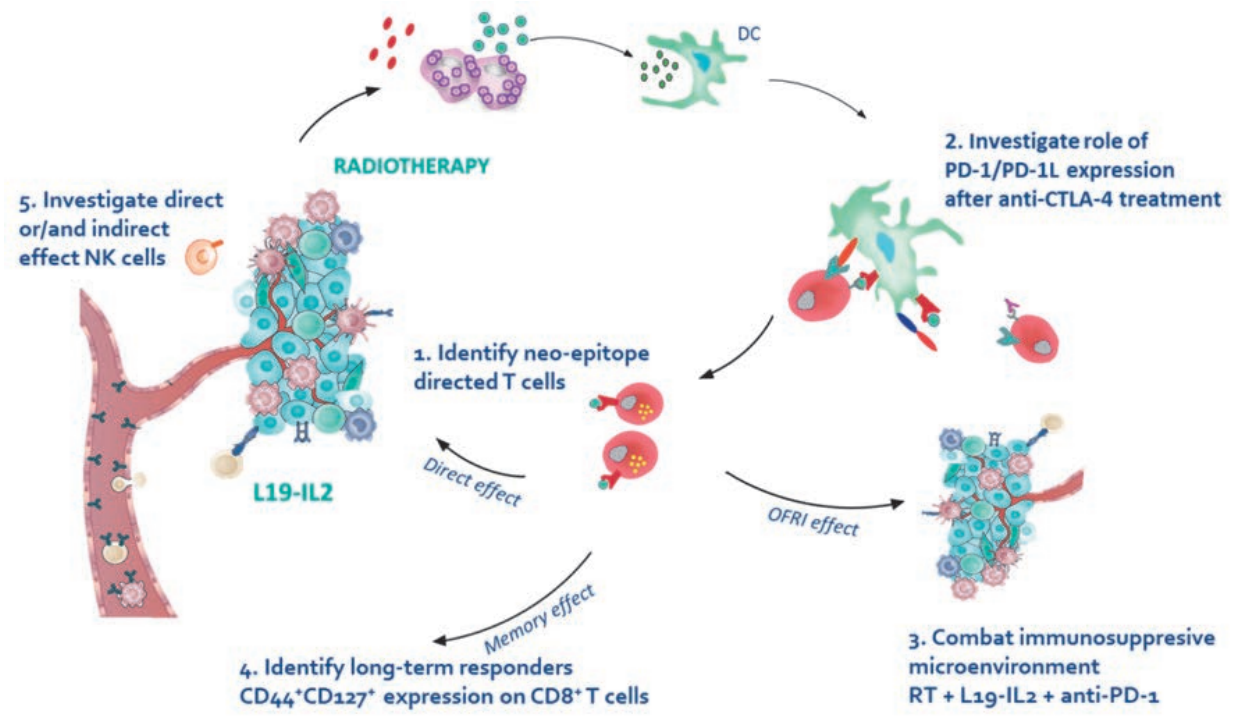

Figure 1: Overview of future perspectives - this dissertation.

1. The identification of neo-eptitope directed T cells and creation of personalized immune profiles (chapter 3 ,

$4,5)$

2. The investigation of the role of PD-1/ PD-L1 as trimodal treatment resistance mechanism (chapter 8)

3. Investigate the role of PD-1 in the OFRI effect and the possibility to increase the OFRI effect using RT + L19IL2 + anti-PD-1 treatment (chapter 5)

4. Investigate possibility to identify long-term responders based on $\mathrm{CD} 44^{+} \mathrm{CD} 127^{+}$expression on $\mathrm{CD} 8+\mathrm{T}$ cells (chapter 5)

5. Investigate the effects of RT + L19-IL2 treatment in tumours with varying expression of $\mathrm{MHCl}$ (chapter 6)

\section{FUTURE PERSPECTIVES - A BROADER VIEW}

Based on recent ground-breaking findings (84-87) and their totally different way of treating cancer by targeting the tumour indirectly via the immune system, a major 
change of paradigm has been started (83). At this moment, we are right in the middle of this immunotherapeutic revolution. Profound progress in immunotherapy research was achieved in for example the field of checkpoint inhibitors (88), adoptive cell therapy (including genetically engineered lymphocytes that express conventional $\mathrm{T}$ cell receptors or chimeric antigen receptors) (93) and immune modulating cytokines (including IL2 (94)). The recent advances in cancer immunotherapy clearly illustrate long-term clinical benefit in some advanced cancer patients, and the potential for cure is realistic (95). Considering the total burden of cancer, and the rising possibilities to successfully manipulate the anti-tumour immune response, the few FDA approved antibodies (as monotherapy) can in my opinion be considered as 'tip of the iceberg'. In addition, as described in this dissertation and recognized by others $(84-87,91,96)$, even better clinical responses can be achieved when therapies are combined with each other, or with RT. Furthermore, it has been recognized that hypoxic regions present in a tumour can prevent adequate anti-tumour immune responses (26) and that increasing tumour oxygenation can improve RT efficacy $(97,98)$. Therefore, the use of hypoxia targeting treatment (99) to specifically target hypoxic areas in solid tumours or strategies to overcome tumor hypoxia, might hold promise to further increase therapeutic potential of immunotherapies and/or radiotherapy. With all these novel immunotherapeutic approaches, which can be used in combination with other treatment modalities or different RT schedules, the diversity of possible treatment options is almost unlimited. Therefore, in my opinion, cancer research should even more be executed in a 'bench-bedside-bench' approach, and the focus at this moment should be on the 'bedside-bench' part. However, the bench needs to be seen in a broader perspective: the collaboration between biologists, oncologists, immunologists, data scientists, mathematicians and medical engineers, will become even more crucial the coming decennium. Together, we need to develop a biomarker model, which integrates biological information from tumours (28), the anti-tumour immune response $(44,48)$, and other important parameters (including for example microbiota (100-104)) into a mathematical framework or platform (105) able to predict an accurate antitumour immune response, which can be used to select patients for optimal treatment. 


\section{REFERENCES}

1. Demaria S, Golden EB, Formenti SC. Role of Local Radiation Therapy in Cancer Immunotherapy. JAMA Oncol. 2015;1(9):1325-32.

2. Zegers CM, Rekers NH, Quaden DH, Lieuwes NG, Yaromina A, Germeraad WT, et al. Radiotherapy combined with the immunocytokine L19-IL2 provides long-lasting antitumor effects. Clin Cancer Res. 2015;21(5):1151-60.

3. Menrad A, Menssen HD. ED-B fibronectin as a target for antibody-based cancer treatments. Expert Opin Ther Targets. 2005;9(3):491-500.

4. Kaczmarek J, Castellani P, Nicolo G, Spina B, Allemanni G, Zardi L. Distribution of oncofetal fibronectin isoforms in normal, hyperplastic and neoplastic human breast tissues. Int J Cancer. 1994;59(1):11-6.

5. D'Ovidio MC, Mastracchio A, Marzullo A, Ciabatta M, Pini B, Uccini S, et al. Intratumoral microvessel density and expression of ED-A/ED-B sequences of fibronectin in breast carcinoma. Eur J Cancer. 1998;34(7):1081-5.

6. El-Emir E, Dearling JL, Huhalov A, Robson MP, Boxer G, Neri D, et al. Characterisation and radioimmunotherapy of L19-SIP, an anti-angiogenic antibody against the extra domain $B$ of fibronectin, in colorectal tumour models. Brit J Cancer. 2007;96(12):1862-70. PMCID: 2359968.

7. Pujuguet P, Hammann A, Moutet M, Samuel JL, Martin F, Martin M. Expression of fibronectin ED-A+ and ED-B+ isoforms by human and experimental colorectal cancer. Contribution of cancer cells and tumorassociated myofibroblasts. The American journal of pathology. 1996;148(2):579-92. PMCID: 1861682.

8. Balza E, Carnemolla B, Mortara L, Castellani P, Soncini D, Accolla RS, et al. Therapy-induced antitumor vaccination in neuroblastomas by the combined targeting of $\mathrm{IL}-2$ and TNFalpha. Int J Cancer. 2010;127(1):101-10.

9. Rossin R, Berndorff D, Friebe M, Dinkelborg LM, Welch MJ. Small-animal PET of tumor angiogenesis using a (76)Br-labeled human recombinant antibody fragment to the ED-B domain of fibronectin. Journal of nuclear medicine : official publication, Society of Nuclear Medicine. 2007;48(7):1172-9.

10. Tijink BM, Neri D, Leemans CR, Budde M, Dinkelborg LM, Stigter-van Walsum $M$, et al. Radioimmunotherapy of head and neck cancer xenografts using 1311-labeled antibody L19-SIP for selective targeting of tumor vasculature. J Nucl Med. 2006;47(7):1127-35.

11. Eigentler TK, Weide B, de Braud F, Spitaleri G, Romanini A, Pflugfelder A, et al. A dose-escalation and signal-generating study of the immunocytokine L19-IL2 in combination with dacarbazine for the therapy of patients with metastatic melanoma. Clin Cancer Res. 2011;17(24):7732-42.

12. Johannsen M, Spitaleri G, Curigliano G, Roigas J, Weikert S, Kempkensteffen C, et al. The tumourtargeting human L19-IL2 immunocytokine: preclinical safety studies, phase I clinical trial in patients with solid tumours and expansion into patients with advanced renal cell carcinoma. Eur J Cancer. 2010;46(16):2926-35.

13. Johnson EE, Yamane BH, Buhtoiarov IN, Lum HD, Rakhmilevich AL, Mahvi DM, et al. Radiofrequency ablation combined with KS-IL2 immunocytokine (EMD 273066) results in an enhanced antitumor effect against murine colon adenocarcinoma. Clinical cancer research : an official journal of the American Association for Cancer Research. 2009;15(15):4875-84. PMCID: 2735865.

14. Gutbrodt KL, Schliemann C, Giovannoni L, Frey K, Pabst T, Klapper W, et al. Antibody-based delivery of interleukin-2 to neovasculature has potent activity against acute myeloid leukemia. Science translational medicine. 2013;5(201):201ra118.

15. Golden EB, Frances D, Pellicciotta I, Demaria S, Helen Barcellos-Hoff M, Formenti SC. Radiation fosters dose-dependent and chemotherapy-induced immunogenic cell death. Oncoimmunology. 2014;3:e28518. PMCID: 4106151.

16. Demaria S, Formenti SC. Radiation as an immunological adjuvant: current evidence on dose and fractionation. Front Oncol. 2012;2:153. PMCID: 3481113.

17. Kang J, Demaria S, Formenti S. Current clinical trials testing the combination of immunotherapy with radiotherapy. J Immunother Cancer. 2016;4:51. PMCID: 5028964. 
18. Dewan MZ, Galloway AE, Kawashima N, Dewyngaert JK, Babb JS, Formenti SC, et al. Fractionated but not single-dose radiotherapy induces an immune-mediated abscopal effect when combined with anti-CTLA-4 antibody. Clin Cancer Res. 2009;15(17):5379-88. PMCID: 2746048.

19. Dovedi SJ, Adlard AL, Lipowska-Bhalla G, McKenna C, Jones S, Cheadle EJ, et al. Acquired resistance to fractionated radiotherapy can be overcome by concurrent PD-L1 blockade. Cancer research. 2014;74(19):5458-68.

20. Filatenkov A, Baker J, Mueller AM, Kenkel J, Ahn GO, Dutt S, et al. Ablative Tumor Radiation Can Change the Tumor Immune Cell Microenvironment to Induce Durable Complete Remissions. Clinical cancer research : an official journal of the American Association for Cancer Research. 2015;21(16):3727-39. PMCID: 4537844.

21. Schaue D, Ratikan JA, Iwamoto KS, McBride WH. Maximizing tumor immunity with fractionated radiation. International journal of radiation oncology, biology, physics. 2012;83(4):1306-10. PMCID: 3337972.

22. Rekers NH, Troost EG, Zegers CM, Germeraad WT, Dubois LJ, Lambin P. Stereotactic ablative body radiotherapy combined with immunotherapy: present status and future perspectives. Cancer Radiother. 2014;18(5-6):391-5.

23. Seung SK, Curti BD, Crittenden M, Walker E, Coffey T, Siebert JC, et al. Phase 1 study of stereotactic body radiotherapy and interleukin-2--tumor and immunological responses. Sci Transl Med. 2012;4(137):137ra74

24. Rekers NH, Zegers CM, Germeraad WT, Dubois L, Lambin P. Long-lasting antitumor effects provided by radiotherapy combined with the immunocytokine L19-IL2. Oncoimmunology. 2015;4(8):e1021541. PMCID: 4570128.

25. Postow MA, Callahan MK, Barker CA, Yamada Y, Yuan J, Kitano S, et al. Immunologic correlates of the abscopal effect in a patient with melanoma. N Engl J Med. 2012;366(10):925-31. PMCID: 3345206.

26. Chouaib S, Messai Y, Couve S, Escudier B, Hasmim M, Noman MZ. Hypoxia promotes tumor growth in linking angiogenesis to immune escape. Front Immunol. 2012;3:21. PMCID: 3341970.

27. Sharma P, Allison JP. The future of immune checkpoint therapy. Science. 2015;348(6230):56-61.

28. Schumacher TN, Schreiber RD. Neoantigens in cancer immunotherapy. Science. 2015;348(6230):69-74.

29. Formenti SC, Demaria S. Local control by radiotherapy: is that all there is? Breast cancer research : BCR. 2008;10(6):215. PMCID: 2656907.

30. Demaria M, Ohtani N, Youssef SA, Rodier F, Toussaint W, Mitchell JR, et al. An essential role for senescent cells in optimal wound healing through secretion of PDGF-AA. Developmental cell. 2014;31(6):722-33. PMCID: 4349629.

31. Demaria S, Ng B, Devitt ML, Babb JS, Kawashima N, Liebes L, et al. Ionizing radiation inhibition of distant untreated tumors (abscopal effect) is immune mediated. International journal of radiation oncology, biology, physics. 2004;58(3):862-70.

32. Okuma K, Yamashita H, Niibe Y, Hayakawa K, Nakagawa K. Abscopal effect of radiation on lung metastases of hepatocellular carcinoma: a case report. J Med Case Rep. 2011;5:111. PMCID: 3069951.

33. Wersall PJ, Blomgren H, Pisa P, Lax I, Kalkner KM, Svedman C. Regression of non-irradiated metastases after extracranial stereotactic radiotherapy in metastatic renal cell carcinoma. Acta Oncol. 2006;45(4):493-7.

34. Mole RH. Whole body irradiation; radiobiology or medicine? Br J Radiol. 1953;26(305):234-41.

35. Grass GD, Krishna N, Kim S. The immune mechanisms of abscopal effect in radiation therapy. Curr Probl Cancer. 2016;40(1):10-24.

36. Ehlers G, Fridman M. Abscopal effect of radiation in papillary adenocarcinoma. Br J Radiol. 1973;46(543):220-2.

37. Kingsley DP. An interesting case of possible abscopal effect in malignant melanoma. $\mathrm{Br} \mathrm{J}$ Radiol. 1975;48(574):863-6.

38. Nobler MP. The abscopal effect in malignant lymphoma and its relationship to lymphocyte circulation. Radiology. 1969;93(2):410-2.

39. Ohba K, Omagari K, Nakamura T, Ikuno N, Saeki S, Matsuo I, et al. Abscopal regression of hepatocellular carcinoma after radiotherapy for bone metastasis. Gut. 1998;43(4):575-7. PMCID: 1727260. 
40. Rees GJ. Abscopal regression in Iymphoma: a mechanism in common with total body irradiation? Clin Radiol. 1981;32(4):475-80.

41. Sham RL. The abscopal effect and chronic lymphocytic leukemia. Am J Med. 1995;98(3):307-8.

42. Siva S, MacManus MP, Martin RF, Martin OA. Abscopal effects of radiation therapy: a clinical review for the radiobiologist. Cancer letters. 2015;356(1):82-90.

43. Vatner RE, Cooper BT, Vanpouille-Box C, Demaria S, Formenti SC. Combinations of immunotherapy and radiation in cancer therapy. Frontiers in oncology. 2014;4:325. PMCID: 4246656.

44. Galon J, Mlecnik B, Bindea G, Angell HK, Berger A, Lagorce C, et al. Towards the introduction of the 'Immunoscore' in the classification of malignant tumours. The Journal of pathology. 2014;232(2):199209. PMCID: 4255306.

45. Poleszczuk JT, Luddy KA, Prokopiou S, Robertson-Tessi M, Moros EG, Fishman M, et al. Abscopal Benefits of Localized Radiotherapy Depend on Activated T-cell Trafficking and Distribution between Metastatic Lesions. Cancer research. 2016;76(5):1009-18.

46. Demaria S, Formenti SC. Can abscopal effects of local radiotherapy be predicted by modeling $\mathrm{T}$ cell trafficking? J Immunother Cancer. 2016;4:29. PMCID: 4869282.

47. Schwager K, Hemmerle T, Aebischer D, Neri D. The Immunocytokine L19-IL2 Eradicates Cancer When Used in Combination with CTLA-4 Blockade or with L19-TNF. J Invest Dermatol. 2013;133(3):751-8.

48. Bentzen AK, Marquard AM, Lyngaa R, Saini SK, Ramskov S, Donia M, et al. Large-scale detection of antigen-specific $T$ cells using peptide-MHC-I multimers labeled with DNA barcodes. Nature biotechnology. 2016;34(10):1037-45.

49. Picarda E, Ohaegbulam KC, Zang X. Molecular Pathways: Targeting B7-H3 (CD276) for Human Cancer Immunotherapy. Clinical cancer research : an official journal of the American Association for Cancer Research. 2016.

50. Park SS, Dong H, Liu X, Harrington SM, Krco CJ, Grams MP, et al. PD-1 Restrains Radiotherapy-Induced Abscopal Effect. Cancer Immunol Res. 2015;3(6):610-9. PMCID: 4827718.

51. Taube JM, Anders RA, Young GD, Xu H, Sharma R, McMiller TL, et al. Colocalization of inflammatory response with B7-h1 expression in human melanocytic lesions supports an adaptive resistance mechanism of immune escape. Science translational medicine. 2012;4(127):127ra37. PMCID: 3568523.

52. Borghaei H, Paz-Ares L, Horn L, Spigel DR, Steins M, Ready NE, et al. Nivolumab versus Docetaxel in Advanced Nonsquamous Non-Small-Cell Lung Cancer. The New England journal of medicine. 2015;373(17):1627-39.

53. Motzer RJ, Escudier B, McDermott DF, George S, Hammers HJ, Srinivas S, et al. Nivolumab versus Everolimus in Advanced Renal-Cell Carcinoma. The New England journal of medicine. 2015;373(19):1803-13.

54. Bos PD, Plitas G, Rudra D, Lee SY, Rudensky AY. Transient regulatory T cell ablation deters oncogenedriven breast cancer and enhances radiotherapy. The Journal of experimental medicine. 2013;210(11):2435-66. PMCID: 3804934.

55. Demaria S, Kawashima N, Yang AM, Devitt ML, Babb JS, Allison JP, et al. Immune-mediated inhibition of metastases after treatment with local radiation and CTLA-4 blockade in a mouse model of breast cancer. Clin Cancer Res. 2005;11(2 Pt 1):728-34.

56. Lee $Y$, Auh SL, Wang Y, Burnette B, Meng Y, Beckett M, et al. Therapeutic effects of ablative radiation on local tumor require CD8+ T cells: changing strategies for cancer treatment. Blood. 2009;114(3):589-95. PMCID: 2713472.

57. Huster KM, Busch V, Schiemann M, Linkemann K, Kerksiek KM, Wagner H, et al. Selective expression of IL-7 receptor on memory T cells identifies early CD40L-dependent generation of distinct CD8+ memory $T$ cell subsets. Proceedings of the National Academy of Sciences of the United States of America. 2004;101(15):5610-5. PMCID: 397444.

58. Kaech SM, Tan JT, Wherry EJ, Konieczny BT, Surh CD, Ahmed R. Selective expression of the interleukin 7 receptor identifies effector CD8 T cells that give rise to long-lived memory cells. Nature immunology. 2003;4(12):1191-8. 
59. Lang KS, Recher M, Navarini AA, Harris NL, Lohning M, Junt T, et al. Inverse correlation between IL-7 receptor expression and CD8 T cell exhaustion during persistent antigen stimulation. European journal of immunology. 2005;35(3):738-45.

60. Madakamutil LT, Christen U, Lena CJ, Wang-Zhu Y, Attinger A, Sundarrajan M, et al. CD8alphaalphamediated survival and differentiation of CD8 memory T cell precursors. Science. 2004;304(5670):590-3.

61. Sallusto F, Lenig D, Forster R, Lipp M, Lanzavecchia A. Two subsets of memory $T$ lymphocytes with distinct homing potentials and effector functions. Nature. 1999;401(6754):708-12.

62. Wherry EJ, Teichgraber V, Becker TC, Masopust D, Kaech SM, Antia R, et al. Lineage relationship and protective immunity of memory CD8 T cell subsets. Nature immunology. 2003;4(3):225-34.

63. Carnemolla B, Borsi L, Balza E, Castellani P, Meazza R, Berndt A, et al. Enhancement of the antitumor properties of interleukin-2 by its targeted delivery to the tumor blood vessel extracellular matrix. Blood. 2002;99(5):1659-65.

64. Vivier E, Raulet DH, Moretta A, Caligiuri MA, Zitvogel L, Lanier LL, et al. Innate or adaptive immunity? The example of natural killer cells. Science. 2011;331(6013):44-9. PMCID: 3089969.

65. Rekers NH, Zegers CM, Yaromina A, Lieuwes NG, Biemans R, Senden-Gijsbers BL, et al. Combination of radiotherapy with the immunocytokine L19-IL2: Additive effect in a NK cell dependent tumour model. Radiotherapy and oncology : journal of the European Society for Therapeutic Radiology and Oncology. 2015.

66. Guillerey C, Huntington ND, Smyth MJ. Targeting natural killer cells in cancer immunotherapy. Nature immunology. 2016;17(9):1025-36.

67. Lode HN, Xiang R, Dreier T, Varki NM, Gillies SD, Reisfeld RA. Natural killer cell-mediated eradication of neuroblastoma metastases to bone marrow by targeted interleukin-2 therapy. Blood. 1998;91(5):1706-15.

68. Moschetta M, Pretto F, Berndt A, Galler K, Richter P, Bassi A, et al. Paclitaxel enhances therapeutic efficacy of the F8-IL2 immunocytokine to EDA-fibronectin-positive metastatic human melanoma xenografts. Cancer Res. 2012;72(7):1814-24.

69. Ghasemi R, Lazear E, Wang X, Arefanian S, Zheleznyak A, Carreno BM, et al. Selective targeting of IL-2 to NKG2D bearing cells for improved immunotherapy. Nat Commun. 2016;7:12878. PMCID: 5036003.

70. Becker JC, Pancook JD, Gillies SD, Furukawa K, Reisfeld RA. T cell-mediated eradication of murine metastatic melanoma induced by targeted interleukin 2 therapy. The Journal of experimental medicine. 1996;183(5):2361-6. PMCID: 2192587.

71. Gutbrodt KL, Casi G, Neri D. Antibody-based delivery of IL2 and cytotoxics eradicates tumors in immunocompetent mice. Molecular cancer therapeutics. 2014;13(7):1772-6.

72. van den Heuvel MM, Verheij M, Boshuizen R, Belderbos J, Dingemans AM, De Ruysscher D, et al. NHS-IL2 combined with radiotherapy: preclinical rationale and phase $\mathrm{Ib}$ trial results in metastatic non-small cell lung cancer following first-line chemotherapy. J Transl Med. 2015;13(1):32.

73. Xiang R, Lode HN, Dolman CS, Dreier T, Varki NM, Qian X, et al. Elimination of established murine colon carcinoma metastases by antibody-interleukin 2 fusion protein therapy. Cancer research. 1997;57(21):4948-55.

74. Igney $\mathrm{FH}$, Krammer PH. Immune escape of tumors: apoptosis resistance and tumor counterattack. Journal of leukocyte biology. 2002;71(6):907-20.

75. Vitale M, Cantoni C, Pietra G, Mingari MC, Moretta L. Effect of tumor cells and tumor microenvironment on NK-cell function. European journal of immunology. 2014;44(6):1582-92.

76. Moretta A, Marcenaro E, Sivori S, Della Chiesa M, Vitale M, Moretta L. Early liaisons between cells of the innate immune system in inflamed peripheral tissues. Trends in immunology. 2005;26(12):668-75.

77. Martin-Fontecha A, Thomsen LL, Brett S, Gerard C, Lipp M, Lanzavecchia A, et al. Induced recruitment of NK cells to lymph nodes provides IFN-gamma for $T(H) 1$ priming. Nature immunology. 2004;5(12):1260-5.

78. Malladi S, Macalinao DG, Jin X, He L, Basnet H, Zou Y, et al. Metastatic Latency and Immune Evasion through Autocrine Inhibition of WNT. Cell. 2016;165(1):45-60. PMCID: 4808520.

79. Roberti MP, Julia EP, Rocca YS, Amat M, Bravo Al, Loza J, et al. Overexpression of CD85j in TNBC patients inhibits Cetuximab-mediated NK-cell ADCC but can be restored with CD85j functional blockade. European journal of immunology. 2015;45(5):1560-9. 
80. Mamessier E, Pradel LC, Thibult ML, Drevet C, Zouine A, Jacquemier J, et al. Peripheral blood NK cells from breast cancer patients are tumor-induced composite subsets. Journal of immunology. 2013;190(5):2424-36.

81. Le Maux Chansac B, Moretta A, Vergnon I, Opolon P, Lecluse Y, Grunenwald D, et al. NK cells infiltrating a $\mathrm{MHC}$ class I-deficient lung adenocarcinoma display impaired cytotoxic activity toward autologous tumor cells associated with altered NK cell-triggering receptors. Journal of immunology. 2005;175(9):5790-8.

82. Garbe C. Interleukin-2 as anticancer immunotherapy in the age of checkpoint inhibition. Translational cancer research. 2016;5, Supplement 2.

83. Couzin-Frankel J. Breakthrough of the year 2013. Cancer immunotherapy. Science. 2013;342(6165): 1432-3.

84. Morgan RA, Dudley ME, Wunderlich JR, Hughes MS, Yang JC, Sherry RM, et al. Cancer regression in patients after transfer of genetically engineered lymphocytes. Science. 2006;314(5796):126-9. PMCID: 2267026.

85. Robbins PF, Morgan RA, Feldman SA, Yang JC, Sherry RM, Dudley ME, et al. Tumor regression in patients with metastatic synovial cell sarcoma and melanoma using genetically engineered lymphocytes reactive with NY-ESO-1. Journal of clinical oncology : official journal of the American Society of Clinical Oncology. 2011;29(7):917-24. PMCID: 3068063.

86. Wolchok JD, Kluger H, Callahan MK, Postow MA, Rizvi NA, Lesokhin AM, et al. Nivolumab plus ipilimumab in advanced melanoma. The New England journal of medicine. 2013;369(2):122-33.

87. Yang JC, Haworth L, Sherry RM, Hwu P, Schwartzentruber DJ, Topalian SL, et al. A randomized trial of bevacizumab, an anti-vascular endothelial growth factor antibody, for metastatic renal cancer. The New England journal of medicine. 2003;349(5):427-34. PMCID: 2275324.

88. Pardoll DM. The blockade of immune checkpoints in cancer immunotherapy. Nature reviews Cancer. 2012;12(4):252-64. PMCID: 4856023.

89. Kavanagh B, O'Brien S, Lee D, Hou Y, Weinberg V, Rini B, et al. CTLA4 blockade expands FoxP3+ regulatory and activated effector CD4+ T cells in a dose-dependent fashion. Blood. 2008;112(4):1175-83. PMCID: 2515138.

90. Tang AL, Teijaro JR, Njau MN, Chandran SS, Azimzadeh A, Nadler SG, et al. CTLA4 expression is an indicator and regulator of steady-state CD4+ FoxP3+ T cell homeostasis. Journal of immunology. 2008;181(3):1806-13. PMCID: 2683757.

91. Twyman-Saint Victor C, Rech AJ, Maity A, Rengan R, Pauken KE, Stelekati E, et al. Radiation and dual checkpoint blockade activate non-redundant immune mechanisms in cancer. Nature. 2015;520(7547):373-7. PMCID: 4401634.

92. Schwager K, Hemmerle T, Aebischer D, Neri D. The Immunocytokine L19-IL2 Eradicates Cancer When Used in Combination with CTLA-4 Blockade or with L19-TNF. J Invest Dermatol. 2012.

93. Rosenberg SA, Restifo NP. Adoptive cell transfer as personalized immunotherapy for human cancer. Science. 2015;348(6230):62-8.

94. Rosenberg SA. IL-2: the first effective immunotherapy for human cancer. Journal of immunology. 2014;192(12):5451-8.

95. Emens LA, Butterfield LH, Hodi FS, Jr., Marincola FM, Kaufman HL. Cancer immunotherapy trials: leading a paradigm shift in drug development. J Immunother Cancer. 2016;4:42. PMCID: 4949883.

96. Leavy O. Tumour immunology: A triple blow for cancer. Nat Rev Immunol. 2015;15(5):265.

97. Janssens GO, Rademakers SE, Terhaard CH, Doornaert PA, Bijl HP, van den Ende P, et al. Accelerated radiotherapy with carbogen and nicotinamide for laryngeal cancer: results of a phase III randomized trial. Journal of clinical oncology : official journal of the American Society of Clinical Oncology. 2012;30(15):1777-83.

98. Overgaard J. Hypoxic modification of radiotherapy in squamous cell carcinoma of the head and neck--a systematic review and meta-analysis. Radiotherapy and oncology : journal of the European Society for Therapeutic Radiology and Oncology. 2011;100(1):22-32. 
99. Dubois LJ, Niemans R, van Kuijk SJ, Panth KM, Parvathaneni NK, Peeters SG, et al. New ways to image and target tumour hypoxia and its molecular responses. Radiotherapy and oncology : journal of the European Society for Therapeutic Radiology and Oncology. 2015;116(3):352-7.

100. Pitt JM, Vetizou M, Daillere R, Roberti MP, Yamazaki T, Routy B, et al. Resistance Mechanisms to Immune-Checkpoint Blockade in Cancer: Tumor-Intrinsic and -Extrinsic Factors. Immunity. 2016;44(6):1255-69.

101. Pitt JM, Vetizou M, Waldschmitt N, Kroemer G, Chamaillard M, Boneca IG, et al. Fine-Tuning Cancer Immunotherapy: Optimizing the Gut Microbiome. Cancer research. 2016;76(16):4602-7.

102. Vetizou M, Pitt JM, Daillere R, Lepage P, Waldschmitt N, Flament C, et al. Anticancer immunotherapy by CTLA-4 blockade relies on the gut microbiota. Science. 2015;350(6264):1079-84. PMCID: 4721659.

103. Zitvogel L, Ayyoub M, Routy B, Kroemer G. Microbiome and Anticancer Immunosurveillance. Cell. 2016;165(2):276-87.

104. Zitvogel L, Galluzzi L, Viaud S, Vetizou M, Daillere R, Merad M, et al. Cancer and the gut microbiota: an unexpected link. Science translational medicine. 2015;7(271):271ps1. PMCID: 4690201.

105. Serre R, Benzekry S, Padovani L, Meille C, Andre N, Ciccolini J, et al. Mathematical Modeling of Cancer Immunotherapy and Its Synergy with Radiotherapy. Cancer research. 2016;76(17):4931-40. 
Summary 
The overall aim of this dissertation was to investigate the possibility of transforming the local effects of radiotherapy (RT) into a systemic tumour-targeted therapy, by enhancing its initial immune stimulating effects with the immunocytokine L19-IL2. We investigated the $\mathrm{CD}^{+} \mathrm{T}$ cell mediated anti-tumour effects of RT in combination with L19-IL2 on irradiated tumours (chapter 3 and 4) and on non-irradiated tumours (chapter 5). We additionally assessed the possibility of this combination treatment to induce a protective and long-lasting anti-tumour memory potential (chapter 5) and its capacity to activate an effective NK cell mediated anti-tumour immune response (chapter 6 and 7). Furthermore, in this dissertation we investigated the possibility to combine this bimodal treatment with a checkpoint inhibitor, anti-CTLA-4 (chapter 8). In chapter 9 we evaluated our data by placing them in a broader view and discussed the future perspectives linked to this dissertation.

The first study in this dissertation (chapter 3 ) described the therapeutic potential and related induced anti-tumour immune responses of RT combined with L19-IL2 in three different in vivo murine tumour models: the 4T1 breast carcinoma, Lewis lung carcinoma (LLC), and the C51 colon carcinoma, which have respectively increasing ED-B expression levels. The therapeutic efficacy was found to be dependent on ED-B expression levels, with highly synergistic effects and a cure rate of $75 \%$ in the high ED-B expressing model C51. These data clearly show the necessity of ED-B expression for an appropriate therapeutic benefit of the combination treatment. Since the majority of human cancers express ED-B, it makes this treatment highly applicable in patients. Furthermore, we proved that $\mathrm{CD}^{+} \mathrm{T}$ cells are causally involved in the anti-tumour response of these $E D-B^{+}$tumours and we showed that the therapeutic benefit is RT dose dependent, since lowering radiation dose resulted in a reduced number of cured animals. These findings resulted in the initiation of a Phase I clinical safety study (NCT02086721) combining SBRT and L19-IL2 in patients with oligometastatic solid tumours, which is currently ongoing in our institute. Preliminary results of this trial indicate that the treatment combination is safe for the first dose level tested.

These curative anti-tumour results clearly show the enormous capability of the 'correctly triggered and enhanced' immune response to distinguish irradiated (i.e. damaged) aberrant (i.e. tumours) from healthy tissues and to eliminate these upon recognition. In chapter 4 we described the possible next steps in this preclinical research; from provoking anti-tumour effects outside RT field to novel treatment combination opportunities for further personalization of this therapy.

The possibility to provoke an anti-tumour effect outside the RT field when combined with systemic L19-IL2 treatment was studied in chapter 5 . Anti-tumour effects triggered by RT observed outside the RT field are called 'abscopal effects' ( $a b$ = away from, scopus = target), however, since we investigate the 'extended' abscopal effect by combining it with systemic L19-IL2 treatment, we introduced the 'OFRI effect' (out-of-field 
radioimmune) terminology in this chapter. We proved for the first time that a single RT dose (15Gy) can result in a curative OFRI effect in $20 \%$ of the macroscopic tumours when combined with L19-IL2. We additionally investigated two clinically relevant fractionated RT schedules ( $5 \times 5 G y$ and $5 \times 2 G y$ ), which also induce OFRI effects, though non-curative. The (curative) OFRI effect triggered by 15Gy and L19-IL2 shows an increase of $\mathrm{CD}^{+} \mathrm{T}$ cells at day 4 after RT. However, a depletion study (starting at day 5) revealed that the curative single RT dose triggered and L19-IL2 mediated OFRI effect is caused predominantly by $\mathrm{CD} 8^{+} T$ cells. These data suggest that $\mathrm{CD} 8^{+} \mathrm{T}$ cells are causing the OFRI effect from day 5 onwards and that $C D 4^{+} T$ cells are involved at an earlier stage in this anti-tumour effect. When investigating the specificity using a СT26 mismatched tumour, we observed a growth delay of these tumours, though therapy targeting directly the C51 tumour with radiation in combination with L19-IL2 did not result in elimination of the mismatched CT26 tumours, suggesting that only a part of the tumour (neo)antigens between C51 and CT26 non-irradiated tumours are overlapping. Based on these findings it appears that OFRI effect is tumour specific but since tumours of different origin may share common (neo)antigens, this therapy has the potential to eradicate multiple tumors within a patient.

In addition we showed that $T$ cells infiltrating non-irradiated tumours have more often a regulatory $\left(\mathrm{FoxP}^{+} \mathrm{CD}^{2} 5^{+}\right.$) or exhausted $\left(\mathrm{PD}-1^{+}\right)$phenotype as compared with irradiated tumours from mice treated with the combination treatment. This suggests that the OFRI effect may be improved by combatting this immunosuppressive environment with, for example, an anti-PD-1 checkpoint inhibitor. However, we have clearly shown that only a single RT dose and not fractionated RT can combat this immunosuppressive environment as well, since these irradiated tumours had a significantly lower infiltrate of regulatory and exhausted T cells. Furthermore, we compared 5 x 5Gy + L19-IL2 with $15 G y+$ L19-IL2 and show that the latter had a higher percentage of tumor infiltrating $\mathrm{CD}^{+} \mathrm{T}$ cells and a higher number of circulating $\mathrm{CD} 4^{+} \mathrm{T}$ cells in the blood. These results clearly demonstrate how crucial the RT schedule is in initiating the optimal OFRI effect and show that a single RT dose is the best trigger for a curative OFRI effect in this in vivo model.

Of great importance, we described the capability of mice, cured from C51 tumours by the combination treatment, to develop a long-lasting immunological protection against new tumour formation, months after tumour cure. Treatment alone (without C51 tumours) did not result in this protective effect, proving the necessity of the 'in situ' immunization process. Treatment with surgery combined with L19-IL2 or high dose RT (40Gy) combined with vehicle, showed significantly less protection. These data suggest that RT is the best inducer for this long-lasting protection against tumours and that L19IL2 is of great importance. Of great interest is the finding that the immune system of mice, able to reject tumour formation upon reinjection of these cells, demonstrated a significant increase of $\mathrm{CD}_{4} 4^{+} \mathrm{CD}_{2} 2 \mathrm{~L}^{+}$and $\mathrm{CD} 44^{+} \mathrm{CD} 62 \mathrm{~L}^{-}$expression on $\mathrm{CD} 8^{+} \mathrm{T}$ cells in the 
lymph nodes, indicative for central memory $\mathrm{T}$ cells and effector $\mathrm{T}$ cells respectively. Therefore, we concluded that central memory $T$ and effector $T$ cells in the lymph node compartments are involved in the protection against new C51 tumour formation. Furthermore, the same mice had a high expression of $C D 44^{+} C D 127^{+}$on splenic, lymph nodes and on circulating (blood) $\mathrm{CD}^{+} \mathrm{T}$ cells, which are classified as effector memory $T$ cells. Effector memory $T$ cells were significantly higher in the blood of mice able to reject tumour cells upon re-challenge and therefore these immune cells are systemically associated with an optimal long-lasting protective effect and may serve as (a basis for) biomarker to identify patients who did not develop immunological memory and thus are at risk for tumour recurrence.

RT combined with L19-IL2 can trigger the adaptive $\mathrm{CD}^{+} \mathrm{T}$ cells mediated immune response, targeting irradiated and non-irradiated tumours and preventing the formation of new tumours months after cure and stop treatment. However, tumour cells need to express $\mathrm{MHCl}$ in order to be recognized and killed by $\mathrm{CD}^{+} \mathrm{T}$ cells, and therefore we investigated the anti-tumour potential of this combination therapy in tumours lacking $\mathrm{MHCl}$ expression in chapter 6. Treatment with L19-IL2 alone already resulted in a significant tumour growth delay and showed a significant increase of tumour infiltrating NK cells. Furthermore, no therapeutic gain was observed when RT was delivered during the L19-IL2 treatment schedule (RT day 2, L19-IL2 day 0, 2, 4), but only when RT is delivered prior to L19-IL2, in the same experimental set-up as used before (RT day 0, L19-IL2 day 1, 3, 5). The enhancement of tumour response was associated with an increase in infiltrating NK cells. The difference in treatment efficacy between both schedules might be explained by the fact that L19-IL2 stimulates the proliferation/activation of NK cells, which are killed when the RT dose is delivered during immunotherapy treatment. To what extend this anti-tumour response is directly performed by NK cells, or if they have an indirect role by shaping the tumour microenvironment favoring the adaptive $T$ cells response, is not proven yet. However, findings of this chapter do show the anti-tumour effect of the (RT +) L19-IL2 (combination) therapy in a $\mathrm{MHCl}$ negative tumour model, which is of great interest since tumours tend to have a heterogeneous expression of $\mathrm{MHCl}$ in a clinical setting. Given the fact that RT + L19-IL2 can induce anti-tumour effects outside the RT field (i.e. the OFRI effect) as described in chapter 5 , and this therapy is able to stimulate NK cells as described in chapter 6 , this might hold even more promise for (micro)metastatic tumour control.

In chapter 7 we have expressed our opinion on the role of L19-IL2 in 'the age of the checkpoint inhibitors' as answer on a commentary. We agreed with the statement that the ability of L19-IL2 to activate NK cells creates an interesting alternative for checkpoint inhibitors in case the cytotoxic T cell 'baseline' is insufficient or in case $\mathrm{MHCl}$ is lacking. However, especially because of the long-lasting (memory) potential provoked by the adaptive immune response, we believe and concluded in chapter 7 that the 
primary goal of an immunotherapeutic (combination) approach is to induce an antitumour immune response mediated by $T$ cells. NK cells might have a supportive (indirect) role in this process as described above, and are able to kill $\mathrm{MHCl}$ negative tumour cells (as shown in chapter 6).

Once the anti-tumour immune response contains the right constituents (delivered via the RT immunization) and is amplified using the right immunotherapy (in our models L19-IL2), it can further be personalized using checkpoint inhibitors able to prolong its anti-tumour potential. In chapter 8 we described our approach to combine low dose RT (2Gy) with L19-IL2 and a checkpoint inhibitor (anti-CTLA-4/Ipilimumab) in the same experimental setting. Here, we have observed that treatment with L19-IL2 results in CTLA-4 expressing T cells and that the trimodal treatment (RT + L19-IL2 + anti-CTLA-4) prolongs growth delay as compared with all other schedules, except the bimodal treatment (RT + L19-IL2). Strikingly, we noticed a reduced cure rate in the trimodal treatment. This importantly indicates that, in contrast to our hypothesis, addition of anti-CTLA-4 worsens tumour response. An increase in regulatory $T$ cells is not the explanation for this reduced treatment efficacy and our first preliminary results show that tumour cells express more PD-L1. Therefore, the PD-L1/PD-1 axis might be an immunosuppressive counteract mechanism used by tumours after anti-CTLA-4 treatment in order to escape the immune attack. However, we cannot yet exclude that timing of the anti-CTLA-4 antibody (day 2 in treatment schedule) or the lack of repetitive administration is the cause of these unexpected findings.

In chapter 9 we evaluated the described chapters of this dissertation, discussed recent relevant literature and described what these results hold for the future. It provides the future perspectives that directly resulted from this dissertation, some of which are already ongoing. These include the phase I clinical study (NCT02086721, ongoing) and a phase II clinical study (approved) investigating RT and L19-IL2 in patients with oligometastatic cancer. It additionally includes a planned preclinical (and phase I clinical) study combining RT and L19-IL2 with anti-PD-1 (nivolumab) to investigate the possibility of increasing the OFRI effect for metastatic cancer. It also includes a broader view for future perspectives, stating that cancer research should even more be executed in a 'bench-bedside-bench' approach, with focus on the 'bedside-bench' part. The bench is described as a 'super team'; a collaboration between biologists, oncologists, immunologists, data scientists, mathematicians and medical engineers.

The curative anti-tumour effect observed in this dissertation proves that RT can function as major anti-tumour immune response trigger, which can be enhanced by an immunotherapeutic approach (L19-IL2 in our models), to kill irradiated and nonirradiated tumours, and to prevent tumour formation months after cure. It shows the synergism between both treatment modalities, opening doors for further personalizing in treatment of metastatic disease, with a curative intent. 

Nederlandse samenvatting 
Het ultieme doel van een anti-kanker behandeling is de patiënt te genezen voor lange termijn, zonder dat zijn gezonde weefsels teveel schade oplopen. De meest gebruikte behandelingsmethoden zijn chirurgie, bestraling en chemotherapie. Waar bij chirurgie een maligniteit invasief wordt verwijderd, wordt deze bij bestraling beschadigd door het gericht toepassen van een stralingsdosis. Chemotherapie hanteert een systemische aanpak welke snel delende cellen doodt, waarvoor onder andere de tumorcellen gevoelig kunnen zijn. Recentelijk staat er een compleet andere aanpak om kanker te vernietigen in de spotlights, namelijk de immuuntherapieën. Na de ontdekking van chemotherapieën in de jaren 40, zijn de opkomst van de immuuntherapieën waarschijnlijk de meest veelbelovende en revolutionaire manier om kanker te bestrijden. Immuuntherapieën verschillen in hun manier van aanpak van bovenstaand beschreven behandelmethoden, omdat deze kanker bestrijden door het immuunsysteem te manipuleren. Dit kunnen we tegenwoordig op een steeds verfijndere en gerichtere manier, en de toediening van L19-IL2 is hiervan een veelbelovend voorbeeld. L19-IL2 is een immuuntherapie welke dankzij zijn L19-deel aan ED-B kan binden, aanwezig in het vaatstelsel van de tumor. IL2 is een interleukine, welke door verschillende immuun cellen wordt geproduceerd en onder andere NK en T cellen kan activeren tot proliferatie. L19-IL2 heeft daarom de potentie om het immuunsysteem gelokaliseerd bij de tumor te activeren.

Het algemene doel van deze dissertatie was het onderzoeken van de systemische antitumor immuun gemedieerde effecten bij de combinatie behandeling van (tumor) gerichte bestraling met systemische L19-IL2. Dit is van groot belang, omdat (micro)metastasen zich vrijwel altijd buiten het bestralingsgebied bevinden. Deze worden daarom gemist, wat de verdere groei van de metastasen mogelijk maakt. Het vertalen van de lokale behandelingseffecten van bestraling naar een behandeling die ook systemisch tumoren kan vernietigen, is daarom van groot belang. Aan de andere kant is recentelijk aangetoond dat tumoren na bestraling een vorm van immunogene celdood ondergaan. Bestraling beschadigt de tumor en de vrijgekomen tumor specifieke (neo)antigenen initiëren een immuun reactie, welke de constituenten bevat van de tumor en daarom als een soort 'in situ' vaccinatie fungeert. De immuuntherapie L19-IL2 heeft de potentie om deze initiële anti-tumor immuun reactie verder te versterken en daarom kan een combinatie van beiden resulteren in de creatie van een gepersonaliseerd en heterogeen 'super medicijn'. Dit super medicijn, de verwekte anti-tumor immuun reactie zelf, heeft de potentie gemetastaseerde kanker gericht te vernietigen, ook nadat de behandeling is gestopt, mogelijk met een curatief resultaat.

In hoofdstuk 3 hebben wij de effecten van een dosis bestraling in combinatie met systemische L19-IL2 onderzocht op de bestraalde tumoren met een variërende expressie van $E D-B$. Geen additionele therapeutische verbetering werd geobserveerd in het lage/negatieve ED-B expressie model 4T1 en een additief effect werd geobserveerd in het intermediaire ED-B expressie model LLC. Het model met de hoogste ED-B expressie 
(C51) toonde een sterk synergisme tussen bestraling en L19-IL2, met een genezingspercentage van 75\%. Dit toont aan dat de werkzaamheid van deze combinatiebehandeling afhangt van ED-B expressie, en aangezien ED-B in de meerderheid van de solide humane tumoren aanwezig is, is de toepasbaarheid van deze behandeling groot. We tonen verder aan dat het percentage $\mathrm{CD}^{+}$cytotoxische $\mathrm{T}$ cellen in tumoren met een hoge ED-B expressie verhoogd is na behandeling met bestraling en L19-IL2, en laten zien dat er een causaal verband bestaat tussen de effectiviteit van deze combinatiebehandeling en de aanwezigheid van deze cellen. Een verdere belangrijke bevinding omschreven in dit hoofdstuk is de afhankelijkheid van deze synergistische behandeling van de gegeven stralingsdosis; een verlaging naar 5 en 2Gy verminderde het aantal tumor eliminaties aanzienlijk. De bevindingen van de studie beschreven in hoofdstuk 3 hebben geresulteerd in de initiatie van een fase I klinische studie (NCT02086721), waarbij patiënten met oligometastasen behandeld worden met SBRT en L19-IL2, welke momenteel loopt binnen ons instituut. Tot nu toe laat de studie zien dat de behandeling veilig is en de milde gemelde pijnklachten die patiënten ervaren bij de tumor regio kunnen mogelijk wijzen op de werkzaamheid van deze behandeling.

Zoals beschreven in hoofdstuk 4, openen de bevindingen beschreven in hoofdstuk 3 de deur voor verder onderzoek. Het synergisme beschreven in hoofdstuk 3 is van groot belang, ze laten duidelijk zien dat beide therapieën elkaar kunnen versterken zodat de snelgroeiende EB-B positieve tumoren volledig verdwijnen. In hoofdstuk 4 staat het 'winnende medicijn', de perfect getriggerde en langdurende anti-tumor immuunreactie zelf, centraal. We beschrijven de mogelijkheden voor verder preklinisch onderzoek; van de systemische behandeling van kanker tot interessante combinaties met hypoxie geactiveerde therapieën (in het geval de tumoren hypoxisch zijn) en checkpoint remmers (in het geval het immuunsysteem een immuun suppressief fenotype heeft).

De mogelijkheid van de perfect getriggerde anti-tumor immuun reactie om tumoren buiten het bestralingsgebied te elimineren en zijn capaciteit om langdurige bescherming te geven tegen de vorming van nieuwe tumoren, staat centraal in hoofdstuk 5 . Het elimineren van tumoren buiten het bestralingsgebied is een fenomeen dat weinig geobserveerd wordt, maar klinisch erg interessant is: een lokale behandeling (bestraling) kan op deze manier namelijk systemisch anti-tumor effect hebben. Onderzoek toont aan dat het abscopal effect (ab - weg van, scopus - target) gemedieerd wordt door het immuun systeem. Aangezien wij in onze studie anti-tumor effecten buiten het bestralingsgebied onderzoeken in combinatie met systemische behandeling van L19-IL2, hebben wij een nieuwe terminologie in het leven geroepen, namelijk 'het OFRI effect' (Out-of-field radioimmune). Het OFRI effect is een anti-tumor effect buiten het bestralingsgebied gemedieerd door het eigen immuunsysteem, welke door middel van een immuuntherapie versterkt wordt. In hoofdstuk 5 laten we voor de eerste keer zien dat 1 dosis bestraling (15Gy) gericht op de tumor resulteert in een curatief OFRI effect wanneer gecombineerd met L19-IL2 in 20\% van de niet-bestraalde 
macroscopische tumoren. Wanneer de stralingsdosis in fracties gegeven werd, $5 \times 2 \mathrm{~Gy}$ of $5 \times 5 G y$, observeerden wij ook een OFRI effect, echter zonder een curatief resultaat.

Tumoren buiten het bestralingsgebied van muizen behandeld met een enkele dosis bestraling plus L19-IL2 lieten een verhoging van $\mathrm{CD}^{+}{ }^{+}$geïnfiltreerde T cellen zien (op dag $4)$, wat suggereert dat deze immuun cellen van belang zijn voor het OFRI effect. Wanneer deze cellen geëlimineerd werden vanaf dag 5, resulteerde dit inderdaad in een snellere groei van de niet bestraalde tumoren, echter dit verschil was niet significant. Wel vonden we een significant snellere tumorgroei wanneer de $\mathrm{CD}^{+} \mathrm{T}$ cellen werden geëlimineerd en deze data vormen dan ook het directe bewijs dat het curatieve OFRI effect veroorzaakt wordt door deze cellen. Om de specificiteit van het OFRI effect te onderzoeken, gebruikten we een tweede ED-B positieve tumor (CT26) in dezelfde experimentele opzet. We observeerden eenzelfde groeivertraging van deze mismatchte tumoren, echter geen van deze tumoren kon geëlimineerd worden door het C51 tumor geïnitieerde OFRI effect. Deze resultaten suggereren dat gedeelde (neo)antigenen tussen deze tumoren wellicht als aangrijpingspunt voor de T cellen van het actieve OFRI effect fungeren.

We tonen in hoofdstuk 5 aan dat T cellen geïnfiltreerd in niet-bestraalde tumoren van muizen behandeld met bestraling en L19-IL2 vaker een immune suppressief fenotype hebben in vergelijking met vehicle controle muizen. Onder andere vonden we een verhoogd infiltraat van $\mathrm{PD}-1^{+}$en van regulatoire $\left(\mathrm{Foxp}^{+} \mathrm{CD} 25^{+}\right) \mathrm{T}$ cellen, wat resulteert in tolerantie en uitputting van deze cellen. Recentelijk zijn er een aantal PD-1 remmers, zoals nivolumab, goedgekeurd als nieuw anti-kanker middel voor bepaalde kankersoorten. Onze bevindingen (hoog PD-1 op T cellen) en deze recente ontwikkelingen, geven de mogelijkheid om het OFRI effect op relatief korte termijn zelfs nog te vergroten. Wanneer muizen behandeld werden met bestraling en L19-IL2, bevatte het immuun infiltraat van bestraalde tumoren een zeer laag percentage regulatoire of uitgeputte $\left(\mathrm{PD}-1^{+}\right) \mathrm{T}$ cellen in vergelijking met de niet-bestraalde tumoren. Dit laat zien dat één dosis bestraling op de tumor de samenstelling van het immuun infiltraat kan veranderen van immuun suppressief/regulatoir naar een effectieve immune respons. Fracties bestraling in combinatie met L19-IL2 resulteerde in een hoge expressie van PD-1 ${ }^{+} \mathrm{T}$ cellen geïnfiltreerd in tumoren buiten en binnen het bestralingsgebied, in vergelijking met vehicle controle. Wanneer niet-bestraalde tumoren van muizen behandeld met 15Gy + L19-IL2 en 5 x 5Gy + L19-IL2 direct met elkaar vergeleken werden, bleek de eerste groep meer $\mathrm{CD}^{+}$tumor infiltrerende $\mathrm{T}$ cellen en meer $\mathrm{CD}^{+}$circulerende $\mathrm{T}$ cellen in het bloed te hebben.

Uit deze bevindingen kunnen we concluderen dat niet-bestraalde tumoren van muizen behandeld met bestraling (1 dosis) en L19-IL2 een hoog percentage geïnfiltreerde regulatoire en uitgeputte $T$ cellen bevatten in vergelijking met de bestraling en vehicle controle. Dit immune suppressieve fenotype is ook terug te vinden in zowel de 
bestraalde als niet-bestraalde tumoren van muizen behandeld met fracties bestralingen en L19-IL2 (vergeleken fracties bestralingen en vehicle). Echter, een directe vergelijking tussen één dosis bestraling en fracties wanneer beiden gecombineerd worden met L19IL2, laat zien dat de eerste groep meer $\mathrm{CD}^{+}$tumor infiltrerende T cellen en meer $\mathrm{CD} 4^{+}$ circulerende $T$ cellen in het bloed te hebben.

We laten verder in hoofdstuk 5 zien dat wanneer muizen genezen zijn van hun tumoren door de combinatiebehandeling (bestraling plus L19-IL2), ze niet meer in staat zijn nieuwe tumoren te vormen. Muizen verkrijgen een langdurig immunologisch beschermend geheugen tegen deze tumoren. Deze immunologische bescherming wordt niet gevormd wanneer muizen zonder tumoren de combinatie behandeling ontvangen, wat aantoont dat de tumor inderdaad de basis vormt voor deze 'in situ' vaccinatie. De tumor kan uiteindelijk vertaald worden in een direct (bestraalde tumor), indirect (nietbestraalde tumor) en langdurig (opnieuw geïnjecteerde tumor) anti-tumor immuun effect. Muizen genezen van de tumoren door chirurgie plus L19-IL2 en hoge dosis bestraling plus vehicle, ontwikkelden significant vaker tumoren na her-injectie van tumorcellen. De eerste groep toont aan dat bestraling en de tweede groep toont aan dat L19-IL2 beiden noodzakelijk zijn voor een optimale langdurige immunologische bescherming tegen tumorvorming. Muizen die niet meer in staat zijn tumoren te vormen, hebben een verhoogd percentage centraal geheugen $\mathrm{T}$ cellen $\left(\mathrm{CD} 44^{+} \mathrm{CD}_{62} \mathrm{~L}^{+}\right.$op $\left.\mathrm{CD}^{+}\right)$en effector $\mathrm{T}$ cellen $\left(\mathrm{CD} 44^{+} \mathrm{CD} 62 \mathrm{~L}^{-}\right.$op $\left.\mathrm{CD}^{+}\right)$in hun lymfeklieren. Daarom suggereren wij dat deze cel subsets betrokken zijn bij de bescherming tegen de vorming van nieuwe tumoren. Dezelfde (tegen nieuwe tumorvorming) beschermde muizen, hebben een hoge expressie van $\mathrm{CD}_{4} 4^{+} \mathrm{CD} 127^{+}$op $\mathrm{CD} 8^{+} \mathrm{T}$ cellen in hun milten, lymfeklieren en bloed. Deze cellen worden geclassificeerd als effector geheugen $T$ cellen. Het blokkeren van CD127 had echter geen invloed op het tumor beschermende vermogen van de muizen. Dit laat zien dat CD127 wel sterk geassocieerd is met het vermogen van de muizen om beschermd te zijn tegen tumoren, maar dat dit beschermende vermogen niet afhankelijk is van CD127. Aangezien de effector geheugen $T$ cellen in het bloed van muizen welke bescherm zijn tegen de vorming van nieuwe tumoren verhoogd is, zouden deze cellen als biomarker kunnen gaan fungeren om een (niet) langdurig immunologisch anti-tumor potentiaal te op te sporen.

De hoofdstukken 1 tot en met 5 richten zich op de $\mathrm{T}$ cel gemedieerde anti-tumor immuun response, echter, in hoofdstuk 6 laten we zien dat bestraling en L19-IL2 ook een NK gemedieerde anti-tumor immuun response kunnen induceren. In dit hoofdstuk maken we gebruik van een $\mathrm{MHCl}$ negatief, maar ED-B positief, tumor model (F9 teratocarcinoom). In de literatuur staat omschreven dat tumoren zonder $\mathrm{MHCl}$ expressie inderdaad als alternatief mechanisme door NK cellen kunnen worden geëlimineerd. In dit hoofdstuk hebben we twee behandelingsschema's gebruikt. Behandeling met alleen L19-IL2 laat een significante tumorgroei vertraging zien. Dit is in lijn met de literatuur welke laat zien dat NK cellen niet noodzakelijk geïmmuniseerd 
hoeven te worden (door bijvoorbeeld bestraling) voor hun activatie. Geen additionele tumorgroei vertraging werd geobserveerd wanneer bestraling in het midden van het L19-IL2 schema gegeven werd (bestraling dag 2, L19-IL2 dag 0, 2, 4). Het behandelingsschema startend met bestraling (bestraling dag 0, L19-IL2 dag 1, 3 en 5) vertraagt de tumorgroei significant. Mogelijk kan dit verschil verklaard worden doordat bestraling gegeven op dag 2 de door L19-IL2 gestimuleerde NK cellen beëindigt. Inderdaad laten we in hoofdstuk 6 zien dat de anti-tumor reactie van de $\mathrm{MHCl}$ negatieve tumoren geassocieerd is met een verhoogd percentage tumor infiltrerende NK cellen. Deze bevindingen zijn van groot belang, in de praktijk zullen tumoren namelijk een heterogene expressie hebben van $\mathrm{MHCl}$ en de uitgevoerde studies laten zien dat bestraling plus L19-IL2 niet alleen een T cel response kan induceren, maar ook een NK cel response. Dit vergroot de toepasbaarheid van deze therapie. Zoals ook omschreven wordt in hoofdstuk 7, is de mogelijkheid van deze combinatiebehandeling om NK cellen te activeren tot het elimineren van tumorcellen zeer voordelig vergelijken andere therapieën, zoals checkpoint remmers, welke alleen maar het adaptieve immuunsysteem kunnen moduleren. Echter concluderen we in dit hoofdstuk dat een optimaal geïnduceerde anti-tumor immuun effect van adaptieve origine de meeste potentie heeft, vooral gezien zijn langdurende bescherming tegen nieuwe tumorvorming. Echter, de indirecte rol van NK cellen om het adaptieve immuunsysteem te moduleren, is wellicht cruciaal voor een optimaal eindresultaat.

In hoofdstuk 3 laten we zien dat bestraling plus L19-IL2 een curatieve en langdurende $\mathrm{CD}^{+} \mathrm{T}$ cel gemedieerde immuun response kan mediëren, welke curatief is in bestraalde tumoren. In hoofdstuk 5 laten we zien dat het verhogen van de bestralingsdosis naar 15Gy (plus L19-IL2) resulteert in een curatief effect in 100\% van de bestraalde tumoren. In plaats van de bestralingsdosis verhogen, is het ook een optie om een lage dosis bestraling (immuun initiërende trigger) te combineren met L19-IL2 (immuun response versterker) en een checkpoint inhibitor (haalt de rem van de T cellen af/verlengt de anti-tumor immune response), om zo de mogelijke stralingsdosis afhankelijke gezonde weefseltoxiciteit te beperken. In hoofdstuk 8 hebben we daarom een behandeling van bestraling (2Gy), L19-IL2 en anti-CTLA-4 (ipilimumab) onderzocht in het ED-B positieve C51 colon carcinoom model. Onze hypothese was dat toevoeging van anti-CTLA-4 aan de bimodale behandeling in een extra groeivertraging of eliminatie van de tumoren zou resulteren. Echter vonden wij dat toevoeging van anti-CTLA-4 resulteerde in een significant minder anti-tumor effect dan behandeling met de bimodale therapie zonder anti-CTLA-4. Geen verschil in circulerende regulatoire $T$ cellen kon worden geobserveerd. De eerste resultaten wijzen op een toename van PD-L1 expressie van tumorcellen na de trimodale behandeling, wat mogelijk als een belangrijk immune suppressief ontsnappingsmechanisme kan fungeren. Echter, het is ook mogelijk dat de gekozen timing van de anti-CTLA-4 injectie (dag 2) niet optimaal is in dit schema. 
Samenvattend laat deze dissertatie zien dat bestraling in combinatie met L19-IL2 kan resulteren in een $\mathrm{CD}^{+} \mathrm{T}$ cel en NK cel gemedieerde anti-tumor immuun response, afhankelijk van het model. Het laat de mogelijkheid van deze behandeling zien om de $\mathrm{CD}^{+} \mathrm{T}$ cel response te activeren zodat deze zelfs tumoren buiten het bestralingsgebied kunnen elimineren en maanden na genezing (en beëindiging van de therapie) kunnen voorkomen dat nieuwe tumoren gevormd worden. Dit immunologische beschermingsmechanisme wordt zeer sterk geassocieerd met de systemische aanwezigheid van effector geheugen $\mathrm{T}$ cellen, welke mogelijk als interessant klinisch uitleessysteem (biomarker) kunnen gaan fungeren. Deze studies laten zien dat het mogelijk is het immuunsysteem met bestraling en een immuuntherapie (L19-IL2) volledig te moduleren in een zeer veelzijdige anti-tumor response. Deze anti-tumor response heeft het geweldige potentieel vertaald te worden naar patiënten, met als uiteindelijk doel gemetastaseerde kanker te gaan behandelen met een curatieve intentie. 

Valorisation addendum 
In this thesis the long-lasting, protective and curative anti-tumour effects of radiotherapy (RT) in combination with the immunocytokine L19-IL2 has been successfully demonstrated in preclinical models. To have societal impact, this knowledge needs to be translated from preclinical (mice) studies to clinical (human) trials to eventually result into new and applicable treatment options. This translational process from findings in the lab towards new patient treatment options is complex and it often takes decades to be successfully achieved. Furthermore, it is mostly a highly disappointing process: despite successful preclinical results, around $85 \%$ of early clinical trials investigating novel drugs fail and only half of the successful therapies will eventually be approved. Therefore, it is important to keep the route from 'preclinical findings' to 'a new treatment option' as short and straightforward as possible. To ensure that the successful preclinical results as described in this dissertation have the potential to create impact as fast as possible, several key elements favouring this translational process are worth to mention. First of all, Maastro clinic has extended expertise in treating patients with (stereotactic ablative) RT, a necessary component of the tested novel treatment strategy. Second, both Maastro lab and Maastro clinic have a long history of conducting translational research leading to clinical trials. At this moment several phase I clinical studies are recruiting patients [NCT00691548; NCT00777894 and NCT00409994] and three studies are closed with follow-up [NCT00704600; NCT00409994 and NCT00969657]. In addition, L19-IL2 as monotherapy has already shown to be safe and effective in several clinical trials. Therefore research does not need to 'start from scratch', which can catalyse the translation process from successful preclinical findings towards the patients. The combination of these key elements made it possible that the preclinical results described in this dissertation already lead to the initiation of a phase I clinical study [NCT02086721], the funding of a phase II clinical trial and initiation of a trimodal study, combining RT and L19-IL2 with anti-PD-1. The knowledge described in this dissertation is therefore already being translated into applicable treatment options, though still in study design, and will hopefully result in a new and curative treatment option for patients with (oligo)metastatic disease in the near future.

\section{CLINICAL RELEVANCE}

Cancer is one of the leading causes of death worldwide, accounting for 8.2 million deaths in 2012. A major clinical challenge is the management of metastatic cancer, which is responsible for $90 \%$ of all cancer deaths. Patients with metastatic cancer mainly rely on palliative treatment and current treatment strategies result in a disappointing median progression-free survival (only 2-12 months for example in metastatic NSCLC). Therefore, there is a high need to develop new effective treatment strategies for these patients. The combinational treatment strategy investigated in this dissertation is of 
great clinical relevance. First of all, tumour irradiation will initiate an anti-tumour immune response containing the elements of a patient's own irradiated tumour. Therefore, combination of RT and immunotherapy based treatments has the potential to trigger a personalized (in situ generated) 'medicine'. In chapter 3 curative antitumour results are described, showing the importance of ED-B presence in the tumour vasculature prior to treatment. Since ED-B expression is restricted predominantly to tumours and it is present in the majority of tumours, most patients with solid tumours may potentially benefit from the treatment combination with little adverse effects as L19-IL2 is a tumour targeted therapy. Furthermore, as described in chapter 5, RT delivered to one tumour can additionally trigger curative anti-tumour effects outside the RT field (OFRI effect). Therefore, this locally triggered combination treatment has the potential to act as an effective systemic therapy. This makes the treatment of great interest for patients with metastatic disease. Last, this combination treatment triggers a long-lasting anti-tumour (memory) immune response. This is of great clinical relevance, since this memory effect enables to target tumour-associated antigens and thus protect patients from recurrences and potentially from development of new tumours.

\section{GAIN FOR SOCIETY}

The successful translation of preclinical data described in this dissertation toward society would, firstly and most importantly, benefit a great variety of (metastatic) cancer patients. As described above, treatment options for these patients are limited and therefore new therapeutic approaches are urgently needed. Introducing a new (combination) treatment for these patients will be highly beneficial for this large group of patients, since they will have the potential to be treated with curative intend instead of receiving palliative therapies. Furthermore, the use of L19-IL2 instead of IL2, to specifically target IL2 to tumours, makes this treatment less toxic. Patients may therefore experience a higher quality of life, which is a major gain for society. Additionally, the long-term anti-tumour effects observed in our studies show that the triggered anti-tumour response can prevent tumour formation months after termination of therapy. The combination treatment may therefore 'learn' the patients' immune system what to target, and these long-living pool of memory $T$ cells have the ability to remember their targets months after cure prolonging uncomplicated patients' life. Next, medical doctors will have more opportunities to treat their patients, although proper patient selection is necessary before initiating treatment. Preclinical results described in this dissertation show that the combination treatment is effective in ED-B positive tumour models. Therefore, the development of a biomarker selecting patients based on the intratumoural ED-B expression prior to treatment, would additionally be a major benefit for society. This way, patients with a high chance to benefit from treatment can be selected and patients with a low chance of benefit could receive 
alternative therapies. This prevents using ineffective treatments and thus decreases the costs. We additionally showed that $\mathrm{CD}^{+}$cytotoxic $T$ cells are highly increased inside tumours that can be eliminated due to the combination treatment and that mice able to prevent new tumour formation have a high level of circulating memory $T$ cells as compared with control groups. All of these findings have, once translated into clinical use, the potential to monitor patients during treatment and adapt or extend treatment when necessary. This increases the treatment efficacy and therefore it provides another gain for society. The initiation of the phase I clinical study in our institute [NCT02086721] can be seen as the first step to create impact in society.

\section{IMPROVEMENT IN HEALTH CARE}

The successful translation of the described preclinical results will greatly improve the health care for (metastatic) cancer patients. It is expected that this novel bimodal treatment modality will increase cure rates as well as control metastatic disease thereby prolonging patients' life. The efficacy of this treatment approach needs to be proved in clinical trials which have been already initiated in our department. Additionally, several (immunological) parameters (ED-B expression, increase in CD8+ T cells) associated with elimination of irradiated and non-irradiated tumours when combined with L19-IL2 as well as the presence of memory T cells in long-term protected mice, have the potential to be translated into predictive biomarkers. These biomarkers could be implemented and used for the selection and monitoring of patients in the near future. Therefore, health care will be improved directly, because a new treatment option will be provided. Indirectly, the implementation of new predictive biomarkers to select patients, will have a big advantage since only potential responders will receive treatment. Other treatments, more effective in the cohort of non-responders or similarly effective but less expensive, will be offered to non-responders. This will reduce costs of the treatment and will spare these patients from possible therapy induced side-effects.

\section{NOVELTY OF CONCEPT}

The conversion of RT as a local treatment into an effective systemic treatment can be seen as the main conceptual novelty. It is known that the effects of RT on the tumour can initiate an anti-tumour immune response, however, this seldom results in the systemic regression of un-irradiated tumours. Using a novel immunotherapeutic approach, the immunocytokine L19-IL2, we are able to further strengthen the RT induced immune response. In our preclinical models, this resulted in the elimination of not only irradiated but also a part of non-irradiated tumours. Additionally, this immune response was able to prevent new tumour formation months after cure. The novelty of 
the concept described in this dissertation relies on the interplay between RT and L19IL2, which may eventually result in a new treatment opportunity for patients with (metastatic) disease.

\section{ROAD TO THE MARKET}

The phase I clinical study (NCT02086721, animation available: https://youtu.be/ $\mathrm{xHbwQuCTkRc)}$ is currently ongoing in our institute and will primarily investigate the safety of the combination L19-IL2 with SABR in patients with oligometastatic solid tumours..Progression-free survival, local control rate, quality of life, non-invasive response using PET and overall survival are secondary endpoints. Furthermore, based on our findings a phase II clinical trial has been funded (animation available: https://youtu.be/6wDE6RkrikA). This phase II study will investigate the combination of SBRT and L19-IL2 in patients with non-small cell lung cancer (NSCLC). Additionally, the results described in chapter 5, showing the presence of immunosuppressive T cells (PD$1^{+}$) infiltrating tumours outside the RT field, suggest that the RT + L19-IL2 combination treatment might be increased in its therapeutic potential by combining it with an antiPD-1 checkpoint inhibitor (e.g. nivolumab). Currently, we applied for a KWF grant in which we will establish the safety of this trimodal treatment for stage IV non-small cell lung cancer (NSCLC) patients (animation available: https://youtu.be/7ckZeWWyhts). Secondary endpoints in the latter clinical study include overall survival, progression free survival and quality of life. In addition we will include exploratory endpoints to investigate correlations of outcome measures with immunological markers in tumours and blood.

Therefore, the clinical studies as described above, will not only investigate the safety and effectiveness of radiotherapy combined with L19-IL2, the investigated correlations with (immunological) parameters to evaluate therapy response and OFRI effects will be a crucial step on the 'road to the market'. First of all, the development of a fast and reproducible assay for the detection of ED-B can be marketed. ED-B can be detected in tumours tissues (via ex vivo immunohistochemistry) or using a PET probe. Furthermore, ED-B can also be detected in the blood (serum), though clinical studies should reveal if ED-B expression in the blood correlates with therapeutic efficacy. Second the presence of tumour reactive cytotoxic $T$ cells can form the basis for a marketable product. An assay predicting the adequate (OFRI) anti-tumour response based on effector (memory) $T$ cell reactivity against tumour (neo)antigens in the blood, is an interesting concept. It can become a relevant tool to monitor patients during radiotherapy and L19-IL2 treatment, however, it could be implemented as a novel tool for a panel of different immunotherapeutic approaches. Thus far, there is a lack of reliable biomarkers for the use of immunotherapies, such as checkpoint inhibitors, which is a major limitation of 
these therapies. A recent approach to predict treatment outcome based on tumour infiltrating immune cells (Immunoscore) is an interesting development. However, an assay based on tumour reactive T cells in the blood has probably even more potential, because tumour tissue is not always available.

In general, the preclinical results described in this dissertation may open doors to the development of alternative immunocytokines, for example in the absence of low ED-B expression. It may also show that it is possible to repurpose a 'failed' immunotherapy by providing a radiotherapy trigger prior to administration. Taken together, the preclinical research results described in this dissertation will have an important clinical relevance and societal impact when successfully translated to the clinic. The preclinical data already resulted in the initiation and approval of phase I and II clinical studies, which will be used to investigate therapeutic efficacy and will form the basis for identifying blood and tissue biomarkers for the selection and/or monitoring of patients in the near future, which can all be marketed. 
Acknowledgements / dankwoord 
Dat was het dan. Mijn PhD is afgerond en een veelzijdig, leerzaam en interessant tijdperk ligt achter mij. De afgelopen vier jaar stond mijn PhD centraal. Deze kreeg altijd voorrang, woog het zwaarst. Mijn PhD was de rode draad in mijn leven, mijn houvast, mijn doel. Het onderzoek zat ik mijn hoofd en in mijn hart. Maar geen moment met tegenzin! Veelal voelde ik mij als een kind in een grote speeltuin van mogelijkheden. Ik kreeg de mogelijkheid mij volop te ontwikkelen, mocht vele preklinische studies bedenken en uitvoeren, gaf presentaties op grote congressen, volgde leerzame cursussen en ontmoette interessante mensen. Ik heb het geluk gehad door een geweldig team van professionals omringd te worden, waarin kennis, inzicht, wederzijds vertrouwen en respect centraal stonden. Verder had ik gezellige collega's in Maastro Lab, maar was ook kind aan huis in de PhD room van Maastro Clinic. Daarnaast werd ik omringd door mijn lieve familie en vrienden die mij liefhebben en steunen door dik en dun. Pure rijkdom!

Philippe wil ik graag als eerste enorm bedanken. Zijn vertrouwen in mij zorgde ervoor dat ik mocht beginnen aan deze geweldige uitdaging. Zijn inzicht, mogelijkheid tot zien van oplossingen en visie zijn ongekend groot. Waar het onderzoek naar bestraling in combinatie met immuuntherapie tijdens mijn sollicitatie nog in de kinderschoenen stond, zijn de ontwikkelingen de afgelopen jaren razend snel gegaan. Tijdens mijn PhD heb ik veel van hem geleerd, hebben wij vele interessante brainstormsessies gehad, waarin het enthousiasme er veelal vanaf sprong en we uiteindelijk nóg meer hypothesen en mogelijkheden zagen.

Graag wil ik de leden van mijn leescommissie, prof. dr. F. Ramaekers, prof. dr. G. Bos, prof. dr. E. Deutsch, prof. dr. D. de Ruysscher en prof. dr. J. de Vries, bedanken voor hun tijd mijn proefschrift te lezen en beoordelen.

Thanks to all co-authors. Thank you for all your contribution and scientific input.

Een dankjewel voor de mensen van het CPV, Richard, Saskia, Clarice, Rick, Harry. Dank voor jullie goede zorgen.

I would like to thanks Dario Neri (Philogen) for providing us with L19-IL2.

\section{A big thank to Maastro lab!}

Thank you Marc, Kasper, Jan, Carla, Lydie, Linda, Glenn, Lorena, Justina, Nanda, Carolien, Hanneke, Kim, Raymon, Damiënne, Jonathan, Tessa, Sanaz, Sarah and Marco for the scientific discussions, nice environment and all the fun moments we share!

Ludwig, voor werkelijk iedere vraag en ieder vaag hersenspinsel kon ik bij jou terecht! Bij jou vond ik antwoorden, structuur en kwam ik steeds terug bij de kern van het onderzoek. Onze manier van communiceren verschilt enorm en misschien is dat juist de kracht. Nieuwe inzichten zijn ontstaan tijdens onze werkbesprekingen, waar jij gelukkig altijd tijd voor wist vrij te maken. Daardoor heb ik zelden momenten gekend waarop ik geen uitweg meer zag: jij was er om mij de juiste richting op te sturen. Jouw ideeën 
over het onderzoek, jouw input in de opzet van grote experimenten en tijdens het analyseren en interpreteren van de resultaten, waren onmisbaar. Enorm bedankt Ludwig, ik vond de samenwerking super! Ala, thanks a lot for all you support! You provided me with important insights and I really appreciate our (work-related) discussions. Your input in the preclinical studies, data analyses, statistic and the immunofluorescent stainings were crucial. Natasja en Rianne, bedankt voor al jullie hulp. Wat heb ik veel van jullie mogen leren en wat vormden wij een sterk team (al zeg ik het zelf : $p$ ). Herinneren jullie 'De Drie Dol Dwaze Dagen' en het 'Protocolletje van Nicolletje XXL' nog (om iedereen maar even een kijkje in de mCAT keuken te geven)? In mijn enthousiasme vergat ik regelmatig dat een dag maar 24 uur heeft.. gelukkig dat jullie er waren! Waar was dit onderzoek geweest zonder jullie positieve werkhouding en doorzettingsvermogen, vermogen logisch na te denken onder druk, organisatorische talenten en technische skills? Go Go RadiolmmuneDreamTeam! Ga door met dit geweldige werk! Veronica, thanks a lot for all your help and input! Your PhD started a year ago. Immediately, you could help with all the experiments, design additional experiments and set-up FACS analyses. We share our big love: FlowJo (-)! Chapter 5 would not have looked the same without all of your efforts, ideas and analyses! Thanks a lot, also for all our discussions! Good luck with your PhD! Arjan, jaren deelden wij onze kantoorruimte. Je droge humor, enthousiasme en vele hilarische verhalen, maakten mij, Marco en Kranthi altijd aan het lachen (muhuhoehaa!). Daarnaast gaf je mij directe en eerlijke adviezen, waarvoor ik je nog steeds dankbaar ben. Bedankt Ari, veel geluk in de toekomst samen met je prachtige vrouw en kinderen! Tom, Solar 2015, do I need to say more? Bedankt voor de vele gezellige koffiemomentjes en lunch (soepie!) wandelingen op het werk. Succes met het afronden van je eigen PhD! Marike, jij gaat 3 weken na mij promoveren. Hoe enorm veel extra avonden en weekenden hebben we niet samen in de Uni doorgebracht? Nee, promoveren gaat niet van 9 tot 5, iets dat ook jij weet. Bedankt voor het delen van deze ervaring, met alle frustraties maar ook gezelligheid. Ik wens je veel succes met de voorbereiding van je eigen verdediging! Ga daarna lekker reizen, dat heb je enorm verdiend! Sweet Kranthi, your PhD was not always easy. Or was it always not easy?! But, you did not give up! You always kept on fighting, and that is why I admire you. I loved sharing the room with you. Good luck in future and lots of love for you and your (new) family! Simon, een paar weken na jouw succesvolle verdediging is het nu aan mij om te promoveren. Waar we tegelijkertijd begonnen binnen de mCAT groep, een tijd lang dezelfde kantoorruimte deelden, verschilden onze projecten en werkwijze enorm. Jouw structuur, overzicht en geordende werkwijze is iets waar ik nog flink van kan leren! Veel succes in de toekomst Simon! Eloy, your 'blondie' is leaving! Dancing salsa, practicing Dutch-Spanish, babysitting your doggie, enjoying the ski holidays together: it was so nice meeting you! Thanks for the nice time and see you in Copenhagen! Venus, you are such a wonderful person! I enjoyed the long conversations we had. Wish you all the best with the last months of your PhD! Auke, de maanden waarop wij onze kamer deelden, waren enorm gezellig! Bedankt 
Auke, ook voor al je advies over het promoveren. 'Er is een tijd van komen en er is een tijd van gaan en de tijd van gaan is nu gekomen';)! Veel succes in San Diego! Marijke, hoewel we slechts een paar maanden overlap hebben op de werkvloer, heb ik onze gezellige gesprekken en je input in mijn onderzoek erg gewaardeerd. Bedankt! Veel succes met het afronden van je eigen PhD en je postdoc!

Ook wil ik de studenten bedanken voor hun inzet; Dana, Maria en Brian, bedankt! Dana, je hebt Karen en mij enorm geholpen aan het begin van mijn PhD. Bedankt voor al jouw input, het resultaat staat in hoofdstuk 3. Veel succes met je eigen PhD!

Karen, jou wil ik ook enorm bedanken. Ongeveer een jaartje voor mijn komst, zorgde jij voor de eerste verbluffende resultaten. Je was een geweldige collega, intelligent en communicatief erg sterk, kwaliteiten die voor hele interessante discussies en brainstormsessies zorgden. We hebben ook vreselijk veel plezier gehad! Herinner je je Hendrikje 1, 2 en 3 nog? De volle dagen en avonden die we in het lab doorbrachten om hoofdstuk 3 bij elkaar te pipetteren, waarin het 'we zijn bijna klaar!' gevoel zich op manisch-depressieve wijze wist af te wisselen met het 'we zijn echt niet voor 3.00 uur vannacht klaar!' gevoel. Lang leve de automatisering, lang leve dissociator, die mij later het nodige prak- en prutwerk zou besparen. Bedankt Karen, voor het warme welkom dat je mij bood, de brug die je vormde tussen mij (het lab) en Maastro Clinic, je enorme steun en toewijding omtrent dit onderzoek, voor je altijd luisterende oor en al de gezelligheid de afgelopen tijd! Ruben, voor jou moet ik eigenlijk een aparte categorie maken. Een goede vriend, collega en sportmaatje in één! Lekker zonnebaden op een blok beton, koffie leuten (met 99\% pure chocolade natuurlijk), trapjes oprennen, wintersport: we zijn de afgelopen jaren enorm naar elkaar toe gegroeid! Ik heb onze koffiemomentjes tijdens mijn PhD enorm nodig gehad om even kort af te schakelen en te relaxen! Bedankt voor de leuke tijd en veel succes met het afronden van je eigen PhD! Evert, bedankt voor de interessante discussies, jouw waardevolle input in dit onderzoek en de vertaling van deze resultaten naar een klinische studie. Ik hoop dat veel patiënten hiervan profijt mogen hebben. Ik hoop tevens dat we in de toekomst samen kunnen blijven werken.

Lieve collega's van Maastro Clinic: Aniek, Jurgen, Stefan, Timo, Ralph, Evelyn, Sara, Mark, Sean, Wouter, Andre, Skadi, Floor, Cheryl, bedankt voor jullie wetenschappelijke input en de gezelligheid! Veel succes en geluk in de toekomst! Jean, bedankt voor de mooie plaatjes. Rianne Herben bedankt dat ik voor administratieve vragen bij jou terecht kon. Cary Oberije bedankt voor de vele interessante en leuke gesprekken!

Graag wil ik de collega's van de interne geneeskunde bedanken. Wilfred, ook bij jou stond de deur voor mij open. Bij jou kon ik altijd terecht met vragen over een experimentele opzet of over mijn gevonden resultaten. Bedankt voor je betrokkenheid, je advies en de tijd die je voor mij vrijmaakte. Birgit, bedankt voor al je hulp bij de FACS analyses. Lotte, bedankt voor je waardevolle input in de verschillende studies die 
immunologisch van aard waren. Gerard, Michel, Silvie, Niken, Thomas, bedankt voor jullie input tijdens immunologie meetings, waar ik vooral de eerste jaren van mijn PhD op dinsdagochtend aanwezig was.

Mijn PhD bleek tevens een andere uitdaging voor mij in petto te hebben, eentje van sportieve aard. Als invaller van John Paulissen besloot ik, samen met een groep collega's, deel te nemen aan de Roparun 2014. Een estafetteloop van ruim 500 km, waarbij iedere loper in 48 uur ongeveer $65 \mathrm{~km}$ moest afleggen. Dit om zoveel mogelijk geld op te halen voor de zorg van palliatieve kankerpatiënten. Een waanzinnig avontuur, waarbij ik de grenzen van mijn eigen kunnen heb overschreden (sorry teentje, gelukkig sier je mijn linker voet nog en ben je er niet van pure ellende afgevallen). Een avontuur van uitersten, waarbij geweldige euforie zich afwisselden met pure wanhoop. Rinus

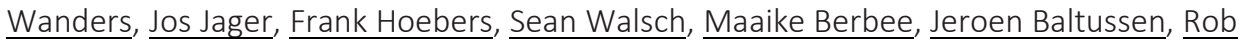
Mannens, Jan Theys, Esther Troost, Simone Pittie, Djoya Hattu, John Paulissen, Lucas Persoon en Marlies Granzier: bedankt dat ik deze geweldige ervaring met jullie mocht delen!

Lieve Claudia, je indrukwekkende verhaal in de bus naar Parijs, over het verlies van je engeltje Mikeela, zit nog altijd in mijn hoofd. Aan de Roparun zal ik nooit meer deelnemen, dat doe ik mijn kleine teentje niet meer aan, maar ik hoop je nog vaak op een feestje tegen te komen, of samen op wintersport te gaan. Succes met MamaLach, dat je een bron van inspiratie en kracht mag blijven voor vele anderen!

Graag wil ik Vincent de Mees bedanken (Animating Science). Vincent, je wist elke keer een geweldige animatie van de wetenschappelijke resultaten te creëren. Je bent erg talentvol in het maken van deze vertaalstap. Tevens bedankt voor het ontwerpen van de cover van mijn thesis, ik ben enorm blij dat je mij dit cadeau doet! Succes met je eigen bedrijf!

\section{Graag wil ik mijn lieve ouders bedanken!}

Mama, waar, wie of wat was ik überhaupt zonder jou geweest? Hoe gelukkig mag ik zijn dat ik jou als moeder heb, mijn grootste steun en toeverlaat. Mijn dankwoord aan jou zou eigenlijk een apart hoofdstuk (of boek) moeten zijn. Met een altijd oprechte trotsheid luisterde je naar mijn verhalen, over mijn nieuwe bevindingen in het lab en over de vele toekomstdromen die ik heb. Altijd weer een andere toekomstdroom, dat dan wel weer, maar inmiddels heb ik mijn koers iets duidelijker bepaald. We hebben een ongekend sterke band en ik ben mij ervan bewust hoe ongelofelijk zeldzaam dat is. De vele telefoongesprekken, wandelingen op zaterdagmiddag, kopjes koffie, lunchmomentjes, vakantie in Griekenland.. Ik heb overal evenveel van genoten. Hoe aangenaam verrast was ik ook als ik na een lange werkdag thuiskwam en er bakjes eten in mijn koelkast op mij stonden te wachten. Lieve Moesch, super bedankt voor al jouw lieve steun, op onbeschrijfelijk veel fronten! Papa, ook jou ben ik erg dankbaar! Als de zachtaardige, stabiele, rustige en nuchtere persoon die je bent, liet je mij meestal 
gewoon ratelen als ik bij jullie thuis kwam en vol enthousiasme over mijn onderzoek begon te vertellen. Maar je was ook de persoon die mij soms tot halt riep als je vond dat de werkdagen echt te belachelijk lang waren. 'Denk je wel een beetje aan jezelf meid?' kon je dan ineens vragen, hetgeen mij liet inzien dat ik een stapje terug moest doen. Maar ik heb niet alleen maar hard gewerkt en daarvan ben jij in maart 2015 getuige geweest, toen je als introducé meeging met een wintersporttripje dat ik voor mijn collega's organiseerde. Het was een topweekend! Geweldige sneeuw, heerlijk zonnetje en een super leuke groep. Lekker samen skiën en daarna een biertje drinken, daar maak je ons toch erg gelukkig mee! Mijn zus Suus, Milian, Luna, Lisa en Noah alias 'de Hilgersjes'! Suus, ook bij jullie stond de deur altijd voor mij open. Beetje gezellig kletsen, stukje hardlopen, wijntjes drinken. Of een avondje relaxen en samen sushi eten (numnumnum)! We lijken als twee druppels water op elkaar, delen dezelfde droge humor en hebben zelden een dag geen contact. Thanks for being my big sisssss (;)). Ik zeg deeeeg! Milian, ook jij bedankt voor al je gastvrijheid. Het was heerlijk om bij jullie wijntjes te drinken in de tuin, een beetje af te schakelen van het werk. Liesje, mijn lieve petekindje! Het is super schattig om te zien waar jouw interesses nu al liggen: bij biologie! Precies zoals je tante Nicolle vroeger kun jij laaiend enthousiast worden van de ontwikkeling van een kikkervisje in een kikker, de vele diersoorten die er op aarde leven en de manier waarop het menselijk lichaam in elkaar zit. Daar komt bij dat je al op jonge leeftijd over een flinke dosis humor beschikt en je een heel slim meisje bent. Ik ben eens benieuwd welk pad jij in je leven mag bewandelen. Vol trots zal ik dan aan de zijlijn staan, jou motiveren, aanmoedigen en steunen, en er altijd voor je zijn! En je weet wat we op jouw achttiende verjaardag gaan doen hè:;?

Graag wil ik mijn lieve vrienden bedanken!

Allereerst een speciaal bedankje aan Philippe Tummers, die helaas niet meer in ons midden is. Bedankt lieve Philippe, voor je gastvrijheid, warme karakter en humor. Het nieuws dat jij een glioblastome multiforme had, sloeg in als een bom. Waar je in het begin nog positief was ('even lekker naar de zonnebank geweest' als we na je bestraling koffie dronken), veranderde dit in een traject net zo grillig als de tumor zelf. Dat vreselijke monster in je hoofd bleek zijn eigen leven te leiden en niet te stoppen. Het liet mij, des te meer, inzien hoe belangrijk het oncologisch onderzoek is.

Lieve vriendinnen, hoe vermoeiend zou mijn PhD geweest zijn, als ik alleen maar gewerkt zou hebben, zonder even tussendoor uit te rusten? Hoewel, uitrusten was er met jullie eigenlijk niet veel bij. Laten we het maar afschakelen noemen (:). Lieve Marieke en Jurate, jullie zijn al jaren geweldige, lieve vriendinnen! Marieke, de laatste jaren deelden wij ons leven voor een volle $100 \%$ en Jurate, onze vriendschap gaat terug naar de tijd dat we uit elkaar gezet werden bij de scheikunde en Duits les op de middelbare school. Jaren riep ik, toen we op carnavalsmaandag in onze apenpakjes over de Minderbroedersberg in Maastricht liepen "hier ga ik ooit promoveren en dan worden jullie mijn paranimfen!". Belofte maakt schuld! Wat ben ik trots dat er zulke 
geweldige vriendinnen aan mijn zijde staan op deze bijzondere dag. Bedankt voor alles, onze vriendschap is goud waard en voor de rest van ons leven, dat weet ik zeker. Lieve $\underline{\text { Suus, }}$ Heleen en Debby, ook wij hebben de deur bij elkaar platgelopen de afgelopen jaren (heb een beeld ;) )! Van wijntjes op vrijdag (of iedere willekeurige avond ;) ), tot zelf sushi maken, een heerlijke $\mathrm{BBQ}$, een gezellige wandeling op zondagmiddag.. zal het ooit gaan vervelen? Bedankt voor al de gezelligheid de afgelopen jaren lieve schatten! Wat delen wij ongelofelijk veel met elkaar en wat genieten we van elkaars gezelschap! Lieve Astrid, jij bent mijn langstlopende vriendschap (vanaf dat we peuters zijn, toch?). We zijn elkaar tijdens onze studies een tijd uit het oog verloren, maar hebben elkaar sinds jaren weer helemaal gevonden! Laura, ook al woon je niet op een steenworp afstand, ik denk dat je de beste gesprekspartner bent die ik mij kan wensen. Je hebt een bijzonder vermogen om te luisteren, mee te denken en te analyseren. Je geweldige en hilarische humor, kent geen grenzen! Ik schiet nog steeds in de lach als ik terugdenk aan onze belevenissen tijdens onze vakantie in Ibiza 2013 (dokter Simon die zijn tekst moest leren, Patty en haar hondenbrokjes, en niet te vergeten: Pablo en de balkonscène. Gouden herinneringen!). Femke, ook al meer dan 10 jaar in mijn leven! Met een mooi huis, een lieve vriend en een engel van een dochtertje heb je het goed voor elkaar! Ik geniet graag van jullie gezinsleventje en natuurlijk ook van onze gezellige avondjes!

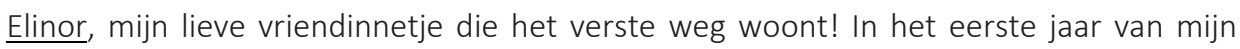
PhD was ik aanwezig op jullie bruiloft in Israël, een ervaring die ik mijn hele leven niet zal vergeten ;) ! Maar ook onze gezellige skypegesprekken en de lieve verjaardagscadeautjes die ik van je ontvang, heb ik erg gewaardeerd! Saskia, mijn andere lieve Biomedische vriendinnetje:-)! Een bezoekje aan Londen zou ik vaker moeten inplannen, want wat hebben we altijd een lol samen! We kunnen elkaar een half jaar niet zien en ons erover verbazen dat het lijkt alsof we elkaar gister nog gezien hebben. Lieve Anne (Versteeg), Sanne, Milou, Laura (Weijers), Anouk, Melanie, Irene bedankt voor de gezelligheid de afgelopen jaren! Sinterklaas vieringen, weekendjes weg (gaan we rattenhol 70 halen, denken jullie?), Koningsdag, verjaardagen en feestjes. Onze gezelligheid kent geen grenzen. Krijn, bedankt voor de gezelligheid de afgelopen jaren! (Ik heb nog een etentje van je tegoed $(;)$ ). Sjoerd, het is nog maar een paar jaar geleden dat ik je leerde kennen in 7even en je hebt mij al enorm weten te inspireren. Bedankt daarvoor! Lizzy en Lisanne, tijdens de cursus 'The lean scientist' leerden wij elkaar kennen en wat hadden wij het leuk samen! Lizzy, succes met je job en Lisanne, succes met je PhD! Anne (van der Meijden): 'Our house, in the middle of the street!' Bedankt voor de gezellige middagjes op mijn dakterrasje en momenten dat we gewoon even snel naar elkaar toe liepen voor een theetje of wijntje. Ongelofelijk hoe twee mensen een huis kunnen delen en elkaar weken niet kunnen zien.. lets met werk en veel uren maken. Anne (Vromen), lief ADHD'tje, wat heb ik genoten van onze drukke gesprekken! Geniet van je sabbatical en je grote liefde. Tessa, net als Anne ken ik jou al jaren en is de vriendschap later ontstaan. Waar bootcamp parkstad wel niet allemaal goed voor is.. (:)! 
Dat brengt mij dan meteen bij de laatste persoon! Het getuigt tevens van een chronologische volgorde om mijn dankwoord met jou af te sluiten Tim! Het was op de valreep van mijn PhD dat jij in mijn leven kwam. In misschien wel de meest stressvolle tijd van mijn leven, een paar weken voor de dissertatie naar de leescommissie gestuurd zou worden, was er eigenlijk geen tijd voor verliefdheid. Om over mijn verdere plannen (twee maanden reizen en voor mijn postdoc naar Kopenhagen verhuizen) nog maar niet te spreken! Bedankt voor de vele geweldige momenten die wij nu al mogen delen! 
Curriculum Vitae 


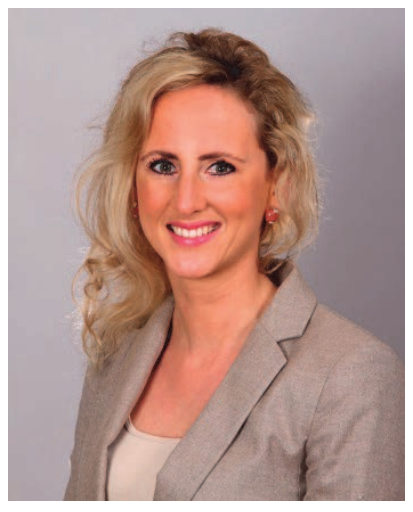

Nicolle Rekers was born on September $1^{\text {th }} 1986$ in Heerlen, the Netherlands. After finishing her preuniversity education (VWO) in 2005 at the Bernadinus College in Heerlen, she started the bachelor Biomedical Sciences at the VU University in Amsterdam, the Netherlands. Her fascination about Immunology and Oncology related research started during her Bachelor and led to an internship at the VU University Medical Center (VUmc), Amsterdam, in the department of Pathology, were she investigated new biomarkers in cervical cancer. After finishing her bachelor, she decided to pursue in the Oncology research field and therefore started the research master Oncology at the VU University, Amsterdam, in 2008. Her master Thesis was rewarded with grade 8.5 , led to her first publication which received an award for best published review article of the journal in 2011. After finishing her master, she worked for one and a half years as research fellow at the VUmc Cancer Center Amsterdam, the Netherlands, were she investigated the role of autophagy in diffuse large B-cell lymphoma. In 2012, Nicolle started her PhD at the department of Radiation Oncology (Maastro Lab) of the Maastricht University, investigating the synergistic interaction between Radiotherapy and Immunotherapy. Interesting research results led to several publications and directly resulted in the initiation of a phase I clinical trial (NCT02086721). In addition, she received several awards during her PhD, including the Wolfsberg Travel Award in 2015 and the Klaas Breur Travel Grant in 2016. Nicolle will continue her scientific carrier at the Technical University of Denmark (DTU), Copenhagen, were she will study the antitumour immune responses and possibilities to use these responses at a large scale as biomarker for selection and/or monitoring of cancer patients, in great detail. 


\section{List of publications}


1. The immunocytokine L19-IL2: a perfect interplay between radiotherapy and long-lasting systemic antitumour immune responses

Nicolle H. Rekers*, Veronica Olivo Pimentel*, Ala Yaromina, Natasja G. Lieuwes, Rianne Biemans, Catharina M.L. Zegers, Wilfred T.V. Germeraad, Evert J. Van Limbergen, Dario Neri, Ludwig J. Dubois**, Philippe Lambin**

Submitted to Nature Communications

2. IL2 based immunotherapies: Towards a personalized and curative antitumor response Nicolle H. Rekers, Veronica Olivo Pimentel, Ala Yaromina, Ludwig Dubois**, Philippe Lambin** Transl Cancer Res 2016;5(Suppl 6):S1292-S1294

3. Radiotherapy, L19-IL2 and ipilimumab trimodal treatment: a suppressive role of PD-L1? Nicolle H. Rekers, Ala Yaromina, Veronica Olivo Pimentel, Ludwig Dubois, Philippe Lambin In preparation for Oncolmmunology

4. Decision support systems for personalized and participative radiation oncology

Lambin P, Zindler J, Vanneste BG, De Voorde LV, Eekers D, Compter I, Panth KM, Peerlings J, Larue RT, Deist TM, Jochems A, Lustberg T, van Soest J, de Jong EE, Even AJ, Reymen B, Rekers NH, van Gisbergen M, Roelofs E, Carvalho S, Leijenaar RT, Zegers CM, Jacobs M, van Timmeren J, Brouwers P, Lal JA, Dubois L, Yaromina A, Van Limbergen EJ, Berbee M, van Elmpt W, Oberije C, Ramaekers B, Dekker A, Boersma LJ, Hoebers F, Smits KM, Berlanga AJ, Walsh S.

Adv Drug Deliv Rev. 2016 Jan 14. pii: S0169-409X(16)30008-4

5. Long-lasting antitumor effects provided by radiotherapy combined with the immunocytokine L19-IL2

Rekers NH, Zegers CM, Germeraad WT, Dubois L**, Lambin P**

Oncoimmunology. 2015 Apr 2;4(8):e1021541

6. New ways to image and target tumour hypoxia and its molecular responses

Dubois LJ, Niemans R, van Kuijk SJ, Panth KM, Parvathaneni NK, Peeters SG, Zegers CM, Rekers NH, van Gisbergen MW, Biemans R, Lieuwes NG, Spiegelberg L, Yaromina A, Winum JY, Vooijs M, Lambin P Radiother Oncol. 2015 Aug 28

7. Combination of radiotherapy with the immunocytokine L19-IL2: Additive effect in a NK cell dependent tumour model

Rekers NH, Zegers CM, Yaromina A, Lieuwes NG, Biemans R, Senden-Gijsbers BL, Losen M, Van Limbergen EJ, Germeraad WT, Neri D, Dubois L**, Lambin P**

Radiother Oncol. 2015 Jun 29

8. Radiotherapy combined with the immunocytokine L19-IL2 provides long-lasting antitumor effects Zegers $\mathrm{CM}^{*}$, Rekers $\mathrm{NH}^{*}$, Quaden DH, Lieuwes NG, Yaromina A, Germeraad WT, Wieten L, Biessen EA, Boon L, Neri D, Troost EG, Dubois LJ**, Lambin P**

Clin Cancer Res. 2015 Mar 1;21(5):1151-60

9. Stereotactic ablative body radiotherapy combined with immunotherapy: present status and future perspectives

Rekers $\mathrm{NH}^{*}$, Troost EG, Zegers CM*, Germeraad WT, Dubois L**, Lambin P**

Cancer Radiother. 2014 Oct;18(5-6):391-5. doi: 10.1016/j.canrad.2014.06.012. Epub 2014 Aug 30

10. Towards tailored therapy of glioblastoma multiforme

Rekers NH, Sminia P, Peters GJ

J Chemother. 2011 Aug;23(4):187-99

\footnotetext{
* Indicates equal contribution

** Indicates equal contribution
} 
